\title{
Measurement of Neutron Spectra in Varied Environments by the Foil-Activation Method With Arbitrary Trials
}
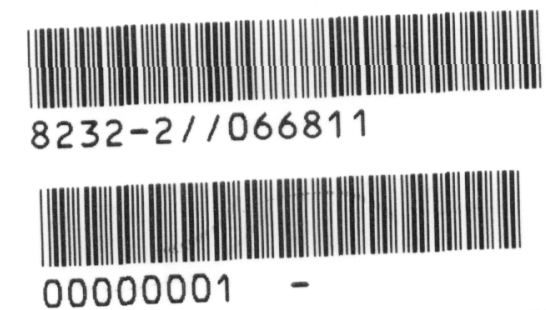

John G. Kelly, David W. Vehar

\section{Prepared by}

Sandia National Laboratories

Albuquerque, New Mexico 87185 and Livermore, California 94550

for the United States Department of Energy

under Contract DE-AC04-76DP00789 


\begin{abstract}
Issued by Sandia National Laboratories, operated for the United States Department of Energy by Sandia Corporation.

NOTICE: This report was prepared as an account of work sponsored by an agency of the United States Government. Neither the United States Govern ment nor any agency thereof, nor any of their employees, nor any of thei contractors, subcontractors, or their employees, makes any warranty, express or implied, or assumes any legal liability or responsibility for the accuracy, completeness, or usefulness of any information, apparatus, product, or process disclosed, or represents that its use would not infringe privately owned rights $\mathrm{R}$ ference here rights. Reference herein to any specific commercial product, process, or service by trade name, trademark, manufacturer, or otherwise, does not necessarily constitute or imply its endorsement, recommendation, or favoring by the United States Government, any agency thereof or any of their contractors or subcontractors. The views and opinions expressed herein do not necessarily state or reflect those of the United States Government, any agency thereof or any of their contractors or subcontractors.
\end{abstract}

Printed in the United States of America Available from

National Technical Information Service

U.S. Department of Commerce

5285 Port Royal Road

Springfield, VA 2216

NTIS price codes

Printed copy: A09

Microfiche copy: A0I 
SAND 87-1330

Unlimited Release

Printed December 1987
Distribution

Category UC-2

\title{
MEASUREMENT OF NEUTRON SPECTRA IN VARIED ENVIRONMENTS \\ BY THE FOIL-ACTIVATION METHOD WITH ARBITRARY TRIALS
}

John G. Kelly

Radiation Physics and Diagnostics Division

and

David W. Vehar

Experimental Systems Design Division

Sandia National Laboratories

Albuquerque, New Mexico 87185

\begin{abstract}
Neutron spectra have been measured by the foil-activation method in 13 different environments in and around the sandia Pulsed Reactor, the white Sands Missile Range Fast Burst Reactor, and the Sandia Annular Core Research Reactor. The spectra were obtained by using the SANDII code in a manner that was not dependent on the initial trial. This altered technique is better suited for the determination of spectra in environments that are difficult to predict by calculation, and it tends to reveal features that may be biased out by the use of standard trial-dependent methods. For some of the configurations, studies have also been made of how well the solution is determined in each energy region. The experimental methods and the techniques used in the analyses are thoroughly explained.
\end{abstract}




\section{ACKNOWLEDGMENTS}

The authors wish to express their sincere appreciation for the very substantial help and support provided by the many who have been involved in this project. D. Berry, C. Harmon, and the other members of the Reactor Applications Division formed the critical link between the experimenters' demands and the accomplishment of the proper dosimeter exposures. C. Holm, who counted the foils and reliably reduced the data to activity levels, provided the essential information on which this work is based. T. Luera and G. Cano provided the encouragement and advice which kept the authors going. Our colleagues in other laboratories provided not only ideas and valuable advice, but also gave us reciprocal experimental data for the interlaboratory comparisons of spectra. In this area we particularly thank W. Sallee, J. Meason, and M. Flanders of the White Sands Missile Range, and C. Heimbach from the Aberdeen Proving Ground. 
I. INTRODUCTION

II. ACTIVATION FOIL EXPOSURES

III. FOIL ACTIVITY MEASUREMENTS 11

A. Beta Counting system 11

B. Gamma-Ray spectrometer system 14

C. Fission Foil Counting 18

IV. DEVELOPING SPECTRA WITH SANDII 25

A. Developing the SPR III Bare Cavity spectrum 28

B. Investigation of Solutions by Perturbation of Activities

C. Investigation of Solutions by Perturbation of Trial Function

D. Interactive Method for Spectrum Adjustment

E. Using the Activity Option of SANDII 61

V. RESULTS AND DISCUSSIONS 63

A. Spectra Measurements 64

(1) SPR III Central Cavity in Free Field (BARNOB6)

(2) SPR III Central Cavity with Polyethylene Liner (POLYCA48)

(3) SPR III Cavity With Aluminum Liner (RAN18) 74

(4) SPR III Leakage spectrum in Free Field at 17 Inches (SLEAK21)

(5) SPR III Between Cadmium-Loaded Polyethylene Blocks--Front Layer Backed with Cadmium sheet (FLATCB24)

(6) Leakage spectrum Between second set of Cadium-Polyethylene and Polyethylene Blocks (CDPOC7) 


$$
\text { CONTENTS - Continued }
$$

(7) SPR III Leakage spectrum Inside Plugged

$\underline{\text { Page }}$ Shield structure (BSHLD20)

(8) SPR III Leakage Spectrum Beam Shield Aperture (BESHLD32)

(9) White Sands Missile Range FBR Leakage spectrum (WHSL25)

(10) ACRR Central Cavity in Free Field (ACRRCF 28)

(11) ACRR Central Cavity with Lead and Boron Shield (ACRPBC14)

(12) ACRR Fuel-Ringed External Cavity (FREC) Spectrum (FREC2344)

(13) ACRR spectrum in New Lead-Boron Cavity Liner (LBACRR12)

B. Goodness of Fit for the Activities

C. Spectrum-Averaged Cross Sections, Activity, and Fluence Determinations

D. Silicon Displacement Damage

E. Comparisons with other spectra

F. Errors

VI. SUMMARY AND CONCLUSIONS

Appendix A The Perturbation Function 160

Appendix B Intersection Point of Perturbed Solutions 163

Appendix C Normalization of Fission-Foil Activities $\quad 165$ 
I. 1 Exposure Geometry for Foils in Cylindrical Cavity

II.2 Boron Ball Geometry 7

III.1 Typical Gamma-Ray Detector Efficiency Curve 19

III.2 Typical Lead Configuration Detector Efficiency Curve

III.3 140 La Activity Versus Time 22

IV.1 Drawing of SPR III 29

IV. 2 WSMR Calculated SPR III spectrum With Low-Energy Tail (Below $1 \mathrm{keV}$ ) Proportional to $\mathrm{E} 32$

IV. 3 First SPR III Cavity spectrum 33

IV. 4 SPR III Cavity spectrum With Thermal Tail 35

IV. 5 Intermediate SPR III Cavity spectrum 36

IV.6 Final SPR III Cavity spectrum 38

IV. 7 Percent Change in Normalized Fluence Caused by $5 \%$ Changes in Activity 41

IV. 8 Perturbation Form 43

IV.9 Fractional Difference Ratio Versus Energy 46

IV.10 The Approach to a Solution 47

IV.11 Convergence of Solutions for Perturbed Trials 48

IV.12 Convergence of Solutions for Perturbed Trials 48

IV.13 First SPR III 17-Inch Leakage spectrum 53

IV.14 Intermediate SPR III 17-Inch Leakage spectrum 54

IV.15 Increasingly Improved Fits with Increasing Dip
at $10^{-2} \mathrm{MeV}$

IV.16 Final SPR III 17-Inch Leakage spectrum from
Flat Trial 


\section{LIST OF ILLUSTRATIONS - Continued}

Figure

Page

IV.17 SPR III 17-Inch Leakage spectrum Calculated bY MORSE

58

IV.18 SPR III 17-Inch Leakage spectrum With MORSE Calculation as Trial

V.1 SPR III Free-Field Cavity spectrum

V.2 Cavity Polyethylene Liner Dimensions 70

V.3 SPR III Cavity spectrum, Polyethylene Liner

V.4 Dimensions of Cylindrical Aluminum Liner and Foil Arrangement

V.5 SPR III Aluminum-Lined Cavity spectrum 78

V.6 SPR III 17-Inch Free-Field Leakage spectrum

V. 7 Configuration of Foil set and Shielding Beside SPR III

V.8 SPR III Leakage Spectrum, Cadmium-Polyethylene

V.9 Configuration for Second Cadmium-Polyethylene and Polyethylene wall structure

V.10 SPR III Leakage Spectrum, Cadmium-Polyethylene and Polyethylene Blocks With Two Trials

V.11 SPR III Leakage spectrum, Cadmium-Polyethylene and Polyethylene

V.12 Drawing of Beam Shield Collimator

V.13 SPR III spectrum in Plugged Beam Shield 98

V.14 Foil Arrangement in Beam Shield Aperature 99

$\begin{array}{lll}\text { V.15 SPR III spectrum in Shield Aperture } & 102\end{array}$

V.16 Foil set Arrangements at WSMR FBR 104

V.17 WSMR Free-Field Leakage Spectrum 107

V.18 ACRR Free-Field Cavity spectrum 111

V.19 Lead and Boron Central Cavity Liner for ACRR 113

V.20 ACRR Spectrum in old Lead-Boron Bucket 115 


\section{LIST OF ILLUSTRATIONS - Continued}

Figure

$\underline{\text { Page }}$

V.21 ACRR Core Fuel Arrangement

118

V.22 Vertical Cross Section Through ACRR Core

V.23 Foil Arrangement in Fuel-Ringed External Cavity 120

V.24 Fuel-Ringed External Cavity spectrum in ACRR

123

V.25 Drawing of New Lead-Boron ACRR Cavity Liner

124

V.26 ACRR Cavity spectrum, New Lead-Boron Liner

127

V.27 Comparison of Fast-Burst Reactor Cavity spectra

V.28 Comparison of Fast-Burst Reactor Leakage spectra

B. 1

Solution Trajectories in Iteration space 
$\underline{\text { Table }}$

$\underline{\text { Page }}$

II.1 List of Foil Reactions 9

III.1 Foil Nuclear Counting Parameters 16

III.2 Fission Yields for selected Nuclides 24

IV.1 Activities of Foils Exposed in SPR III Bare Cavity

IV. 2 Changes in Fluence for $5 \%$ Changes in Activity

IV. 3 Fractional Differences After 15 Iterations for SPR III Cavity spectrum

IV. 4 Extrapolated Fluence Convergence Points 50

IV. 5 Reactions Used for SPR III 17-Inch Leakage spectrum

IV.6 SPR III 17-Inch Leakage Spectrum Calculated by MORSE

V.1 Foil Activities From SPR III in Cavity Free Field

V.2 SPR III Bare Cavity spectrum

V.3 Foil Activities From Polyethylene-Lined SPR III Cavity

V. 4 SPR III Central Cavity spectrum with Polyethylene Liner

V.5 Foil Activities From SPR III Cavity Spectrum With Aluminum Liner

V.6 SPR III Central Cavity Spectrum With Aluminum Liner

V.7 Foil Activities for SPR III 17-Inch

$$
\text { Free-Field Leakage spectrum }
$$

V.8 SPR III 17-Inch Free-Field Leakage spectrum 


\section{LIST OF TABLES - Continued}

Table

Page

V.10 SPR III Leakage Spectrum Between CadmiumPolyethylene Blocks

V.11 Foil Activities for SPR III Leakage spectrum Between Cadmium-Polyethylene and Polyethylene Blocks

V.12 SPR III Leakage Spectrum Between CadmiumPolyethylene and Polyethylene Blocks

V.13

Foil Activities From Plugged Beam Shield Beside SPR III

V. 14

SPR III Leakage spectrum in Plugged Beam Shield

V. 15

Foil Activities From SPR III Leakage spectrum in Beam Shield Aperture

SPR III Leakage spectrum in Beam Shield Aperture Foil Activities for WSMR FBR Leakage spectrum

V. 18

WSMR FBR Leakage Spectrum

V. 19

Foil Activities for ACRR Bare Cavity spectrum

V. 20

ACRR Free-Field Cavity spectrum

V. 21

Foil Activities for Old ACRR Lead-Boron-Lined Cavity

V.22 ACRR Central Cavity spectrum Inside old LeadBoron Shield

V.23 Foil Activities for ACRR Fuel-Ringed External Cavity

V.24

ACRR External Cavity Free-Field spectrum

V. 25

Foil Activities for New Lead-Boron Liner in ACRR Cavity

V.26 ACRR Lead-Boron-Lined Cavity spectrum

V.27 Percentage Deviation of Measured and Calculated Activities 


\section{LIST OF TABLES - Continued}

Table

Page

V.28 Comparison of Old and New Spectrum-Averaged Cross Sections

V. 29

Sulfur and Nickel spectrum-Averaged Cross Sections, spectral Indices, and Hardness Parameters

V. 30

Silicon Displacement Kerma Factors, $K_{D}$, in $\mathrm{MeV} \cdot \mathrm{mb}$ ( $\mathrm{E}$ in $\mathrm{MeV}$ )

V. 31

Spectral Indices and Hardness Parameters from Three Fast-Burst Reactors

C. 1

Corrections to Fission-Foil Activities Based on Nickel-Foil Ratios 


\section{CHAPTER I}

\section{INTRODUCTION}

Accurate determination of the responses of materials and electronic devices to neutron irradiation in a variety of environments is becoming increasingly important because of the accelerating interest in nuclear and space radiation effects. Furthermore, devices often must be tested in radiation fields that do not duplicate the environments for which the devices are intended. For example, components to be used in satellites may be tested at a nuclear reactor. It is imperative, therefore, that the radiation spectrum at the location of the device under test be determined. Models of device response then can be confirmed and used to predict responses in the intended environment.

For the past 25 years, neutron spectrum measurements by the foil-activation method have been carried out in numerous laboratories and have been very successful, following the development of unfolding codes such as SANDII and SPECTRA[1, 2]. The usual unfolding technique is to obtain fluence values at perhaps 600 energy points, spread over 10 orders of magnitude, by measuring the radiation-induced activities of 10 to 20 isotopes that exhibit an array of independent energy-response functions. Because the problem is underdetermined by these traditional unfolding methods, the valid mathematical solutions are unlimited. In SANDII type codes, however, the technique is to restrict the solutions by applying additional physically realistic constraints, such as reasonable smoothness and positive real values for the spectrum at all energy points. Most codes, including SANDII, also require an estimated input spectrum that is based either upon a calculation or upon a measurement in a similar configuration. The solution may not be allowed to vary a great deal from the initial trial. In this situation, the codes do not perform spectrum unfolding--they perform spectrum adjustment.

In SANDII, the trial spectrum at each energy value is multiplied by the neutron reaction cross section of the foil of interest and integrated over all energies to obtain a predicted activity. This procedure is carried out for all the foils in the set that is exposed to the radiation. The calculated and measured activities are then compared. SANDII applies an algorithm developed by Berg and McElroy to alter the trial spectrum so as to obtain a set of calculated activities with a smaller standard deviation (SD) from the measured values in the previous calculation.[1] Iteration in this manner continues until the spectrum obtained provides a specified SD, signaling that an "acceptable" solution has been obtained.

It has long been recognized that this adjustment procedure has serious deficiencies. The solution is not 
unique, and one cannot with certainty determine how close the solution is to the real spectrum. Occasionally, the iteration procedure does not converge, or else it produces a result that seems physically unrealistic. The final result may be heavily dependent on the initial trial, or no physically realistic trial may be available.

For these and other reasons, additional codes have been developed to further restrict the solutions, provide more thorough error analysis, and more effectively approach the real spectrum. [3 - 7] Generally, constraints are added to the algorithm, such as the minimization of curvature, [3] the insertion of a certain functional form in energy regions where sensitivity is low, or the inclusion of weighting factors for measured activities that are poorly determined.

In 1983, a program was initiated to improve the neutron spectrum measurement capabilities at Sandia National Laboratories, Albuquerque (SNLA) by (1) reexamination of the experimental techniques used to obtain foil activities, (2) incorporation of the latest reaction cross-section sets for the "calculation of activities, (3) calibrations at and comparisons with other laboratories, and (4) studies of the various spectrum-adjusting methods to determine their relative success when applied to identical data sets. Significant progress has been made in most of these areas, but the comparison with other unfolding codes has not yet been carried out--primarily because of the considerable success that has accrued from developing a great familiarity with the SANDII code. As a result, this report covers only the results obtained with SANDI I.

The following observations and ideas have guided the conduct of the program thus far:

- A real physical solution does exist, and, unless errors in measurement have been made, all reactions must be compatible with a spectrum. The experimenter is obliged to seek out explanations for activities that do not appear to be compatible, rather than delete them from the set arbitrarily.

- Two of the most important advantages of the activation method are that almost all the reactions used are induced by neutrons only, and they cause the emission of gamma rays in the form of narrow-line spectra. Thus, in the activation counting process, background gamma-ray sensitivity, which could occur during exposure or from delayed gamma rays during counting, is minimized. 
- Care must be taken to obtain accurate and consistent activities for all the reactions of a set, because significant errors can cause very large perturbations in the solutions-particularly in energy regions where only an incorrectly read foil is sensitive. Examples have been noted in which a $20 \%$ activity error caused more than an order-of-magnitude error in the solution at certain energies.

- SNLA personnel have access to a VAX mini-computer and thus have the opportunity to run an adjusting code very frequently. We can rapidly investigate the effects of a multitude of changes in the input data, such as in the trial spectrum or alterations of the activities.

- Although the various unfolding codes have different methods for reaching solutions, most of them calculate the activities in the same way. If, therefore, a trial spectrum is an acceptable solution, the codes will simply verify that fact. Realizing that the algorithms are imperfect, we start from arbitrary trials (flat) and use SANDII to provide clues to better trials. In the end, the code is used to verify a good guess. The code is not expected to provide a solution so much as to suggest how the trial function might be altered for better results. (This is the area in which help from the analyst is needed.) SANDII provides very useful, although at times subtle, information about what must be done to obtain better spectra even when it cannot obtain those spectra without help.

- The experimenters who use SNLA's radiation facilities often require very complicated geometries for their tests, many of which seem very difficult to model well enough to provide realistic calculated trial spectra for an adjusting code. This procedure reduces the reliance on a good initial trial. If the solutions were less dependent on the trial, perhaps spectral structure that resulted from features not taken into account by the model would be revealed. Some discoveries of this type are discussed in this report.

- The Radiation Dosimetry Laboratory was upgraded in a number of significant ways, including improved hardware and procedures. Thus, more reliable activity data are available. The commitment to the foil-activation method was made because of its very great experimental flexibility. It can 
be used with little perturbation of the radiation field, it incurs little or no space penalty in many configurations, and it is not usually sensitive to the angular distribution of the radiation.

- If we could compare the results of spectrum measurements obtained with one counting system for a wide variety of environments, errors in the measurements might be revealed that would not be otherwise evident. For example, measured activities from some reactions are always high with respect to the rest of the foil set. Tendencies like this indicate inaccuracies in the crosssection data, mistakes in the analysis, or real structure in the spectrum that is not properly incorporated into the trial.

These observations have directed the spectrum measurement program up to this time, and they provide a guide for the form of this report. In addition, some procedures have been developed which give a measure of how strongly SANDII seeks a solution at various energies and how much errors in the activities affect the spectrum values.

Although we offer here the latest results of spectra obtained with the currently used techniques, it must be emphasized that source and environment characterization is an ongoing program, and some of the results presented here may be altered by future measurements and analyses. Therefore, it is important for experimenters who may use the SNLA radiation facilities to consult the reactor operators in the preparation of their experiments as to the most recent relevant reactor parameters.

Those readers who are interested only in the results of the experiments may wish to skip chapter II (which deals with the setup and exposure of the foil sets), Chapter III (which describes the Radiation Dosimetry Laboratory procedures), and Chapter IV (which describes the investigations of unfolding or spectrum-adjustment procedures). Chapter $V$ contains summaries and comparisons of the spectrum measurements to date, and Chapter VI discusses planned additional activities. 


\section{ACTIVATION FOIL EXPOSURES}

It is very important to the success of the foilactivation technique that all of the foils be exposed to the same intensity of radiation and the same neutron spectrum. In cases where this is not possible--where there is perhaps a fluence profile over the region where the foils must be positioned (even a few percent variation in some cases)--then these profiles must be measured to provide corrections to the fluence presumed to strike each foil. The profile measurements may have to be made with some detectors that are sensitive at the low-energy end of the spectrum and with others sensitive at the high end. The accuracy of these determinations is very important because the adjusting codes simply cannot fit physically realistic spectra to a set of activities that are incompatible.

To obtain uniformity of exposure, the foil sets are attached to fixtures designed to expose each foil to the same environment in the geometry of interest. For example, in the cylindrical cavities of both the Annular Core Research Reactor (ACRR) and the Sandia Pulsed Reactor III (SPR III), the foils are positioned on a cylindrical surface in two rings at equal small distances above and below the flux centerline of the core (Figure II.1).[8,9] Thus, once cylindrical symmetry of the radiation field is verified, all the foils on this fixture are at the same radius and axial distance from flux maximum. One portion of the foil set is usually exposed in a different manner, however. In both the ACRR and the SPR III cores, the boron ball, which usually contains one nickel foil and four fission foils, is placed on the centerline. In the SPR III bare-cavity experiment discussed in Chapter IV, aluminum wire containing low concentrations of $235 \mathrm{U}$ and linear arrays of nickel foils showed that the centerline fluence was approximately $8 \%$ lower than that at the radius of the other foils. Consequently, the activities of the boron ball foils are multiplied by 1.08 during data reduction. An additional normalization of the fission-foil activities (discussed in Appendix C) is applied later.

The boron balls, illustrated in Figure II.2, are made of sintered $\mathrm{B}_{4} \mathrm{C}$ with a density of $2.5 \mathrm{~g} / \mathrm{cm}^{3}$ and component mass fractions as follows:

Boron

$$
\text { Isotopic } 10_{B}
$$

Isotopic ${ }^{11_{B}}$

Net $10_{B}$
$70.9 \%$

$91.67 \%$

$8.33 \%$

$65.0 \%(0.709 \times 0.9167)$ 


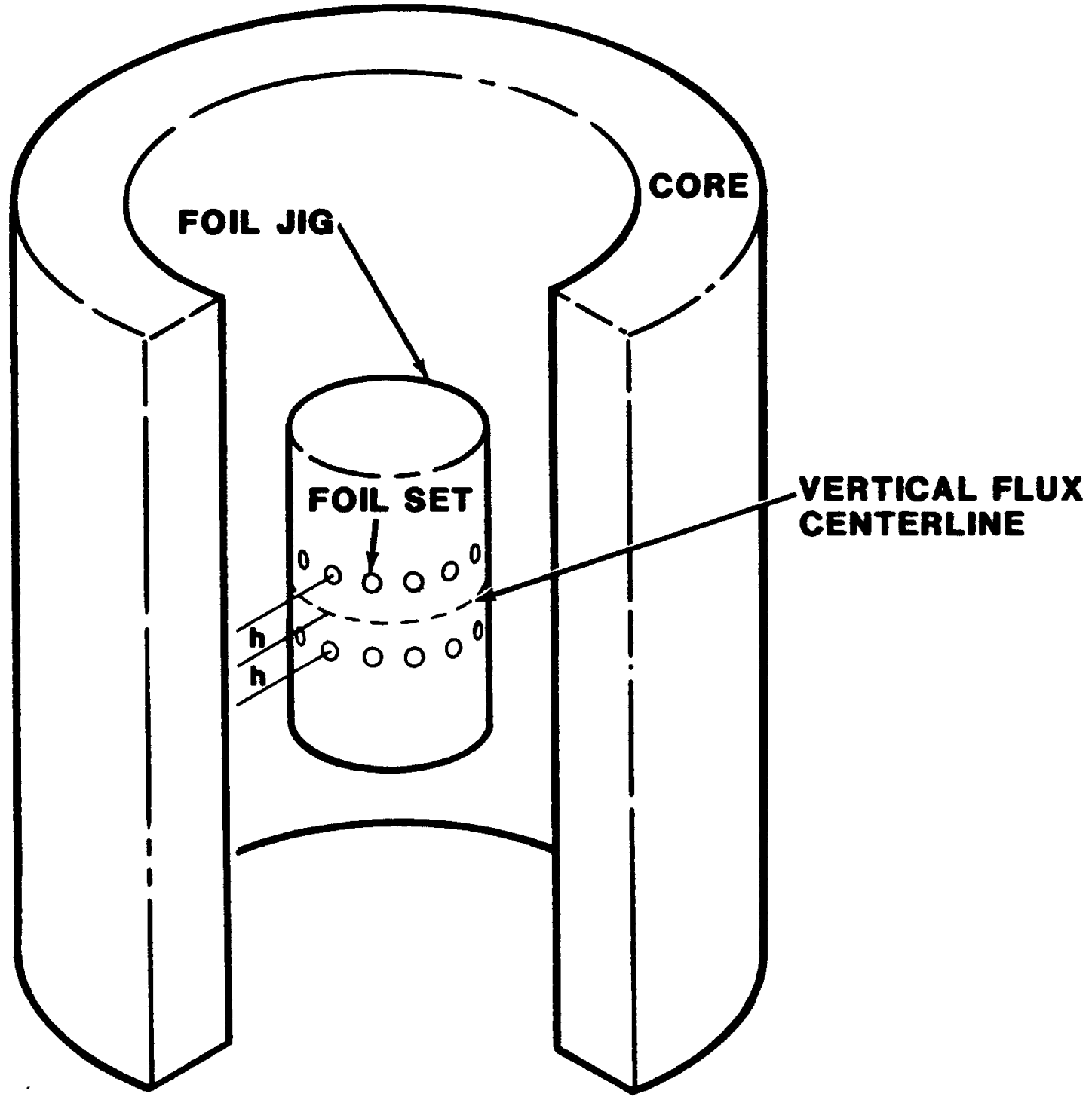

Figure II.1. Exposure Geometry for Foils in Cylindrical Cavity 


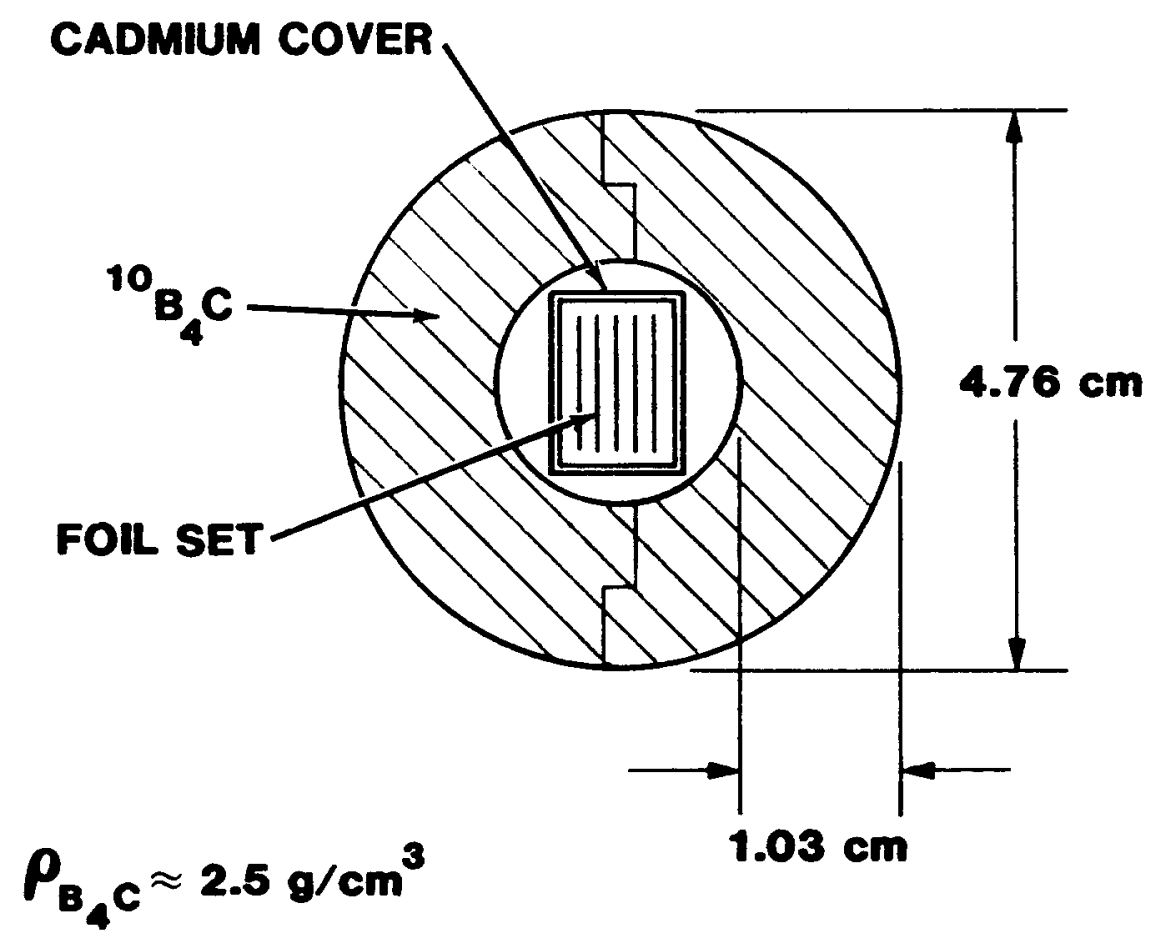

Figure II.2. Boron Ball Geometry

Within the ball cavity, a cadmium pill box (with wall thickness of $4.705 \mathrm{E}-3$ atoms/barn) contains the nickel and the fission foils. During the spectrum-adjustment process, SANDII makes an exponential correction to the activities for the covers on the foils. More is said in chapter IV about the accuracy of this correction.

In most cases, the foils that are exposed outside the boron ball are covered by cadmium shields that are $2.587 \mathrm{E}-$ 3 atoms/barn in thickness.

Tests show that, when a foil set is placed in a confined region near a boron ball, the foils sensitive to neutrons with energy below $10 \mathrm{keV}$ suffer shadowing effects from the ball. Therefore, in the central cavity experiments, we customarily make two exposures--one with the boron ball and a few highthreshold foils, another with a more complete array of foils and no boron ball. The high-threshold foils in the first exposure provide a cross-check on the relative magnitudes of the exposures.

In some cases, a given foil type is exposed both bare and inside a cadmium cover. This is useful with foils such as gold, indium, and sodium, that have response at the thermal end of the spectrum. The two arrangements provide two different response functions. 
There are numerous other configurations for which spectra have been obtained. The objective is to cover a wide variety of useful geometries so as to predict from a broad data base what experimenters may encounter in their particular situations. In addition, some of the geometries have been set up as benchmark configurations for predictions with and comparisons to the results of MORSE Monte Carlo calculations.[10] The benchmark calculations were made by $R$. Sartor and R. T. Perry in 1985, following a careful review of cross sections.[11] The calculations were made for the SPR III reactor leakage spectrum $(43.2 \mathrm{~cm}$ from core center and at 35 inches [88.9 cm] between cadmium-loaded polyethylene blocks). Each configuration is exhibited in detail as the spectrum measurements are discussed in chapters IV and $V$.

Table II.1 lists all of the foil materials and reactions that have been used to produce the spectra that we have measured. Each reaction is listed together with its decay constant, its half-life, and the nominal energy at which its response usually becomes significant. The nominal energy can be very deceptive, depending upon the shape of the spectrum. The CSTAPE column contains the code name recognized by SANDII for each reaction. Occasionally in the text or tables, a reaction will be referred to by its SANDII name (as listed in the CSTAPE column) to simplify the notation. The principal utility of the half-life column $\left(t_{1 / 2}\right)$ is to guide the counting laboratory in choosing the order in which the foils are to be read.

Except for the sulfur and sodium pellets, the foils used in these measurements were all obtained from Reactor Experiments, Inc. Each foil was weighed and accompanied by data on its isotopic composition and impurity levels. The foils were $1.27 \mathrm{~cm}$ in diameter and typically 0.0025 to $0.1 \mathrm{~cm}$ $(0.001$ to 0.05 inch) thick.

The properties of the sulfur pellets are discussed in the next chapter. The sodium foils are salt tablets obtained from Blairex Laboratories, Inc., Indiana.

Not every material listed in Table II.1 is exposed for every test, nor are all materials that are exposed for a given test necessarily used in the spectrum-adjustment procedure. In some cases, a particular activity may not be available, or is too low to be statistically meaningful, or is so far from being compatible with the other reactions that it must be eliminated under the assumption that a counting error has been made that cannot be traced. However, we have learned that, if an error cannot be found, it pays great dividends to work with the spectrum-adjustment procedure until it finally yields a spectrum that is compatible with that reaction. A number of interesting features have been revealed only after the analyst has persevered for some time. 
Table II.1

List of Foil Reactions

\begin{tabular}{|c|c|c|c|c|c|}
\hline $\begin{array}{l}\text { CSTAPE } \\
\text { Name }\end{array}$ & Reaction & $\begin{array}{l}\text { Lambda } \\
(1 / s)\end{array}$ & $t_{1 / 2}$ & Type & Energy \\
\hline IN115G & $\operatorname{In} 115(n, \gamma) \operatorname{In} 116 M \dagger$ & $2.139 E-4$ & $54.0 \mathrm{~m}$ & Th, R+ & $1.46 \mathrm{eV}$ \\
\hline AU197G & $\operatorname{Au} 197(n, \gamma) \operatorname{Au} 198$ & $2.974 E-6$ & $2.70 \mathrm{~d}$ & Th, R+ & 4.9 \\
\hline C059G & $\operatorname{Co59}(n, \gamma) \operatorname{Co60}$ & $4.161 \mathrm{E}-9$ & $5.28 \mathrm{y}$ & Th, R+ & 132 \\
\hline FE58G & $\operatorname{Fe} 58(n, \gamma) \operatorname{Fe} 59$ & $1.783 \mathrm{E}-7$ & $45 \mathrm{~d}$ & $R+$ & 230 \\
\hline MN55G & $\operatorname{Mn} 55(n, \gamma) \operatorname{Mn} 56$ & $7.463 \mathrm{E}-5$ & $2.58 \mathrm{~h}$ & Th, R+ & 337 \\
\hline CU63G & $\operatorname{Cu} 63(n, \gamma) \operatorname{Cu} 64$ & $1.500 \mathrm{E}-5$ & $12.8 \mathrm{~h}$ & Th, R+ & 580 \\
\hline NA23G & $\mathrm{Na} 23(\mathrm{n}, \mathrm{r}) \mathrm{Na} 24$ & $1.286 \mathrm{E}-5$ & $15.0 \mathrm{~h}$ & Th, R+ & $1.71 \mathrm{keV}$ \\
\hline PU239F & $\operatorname{Pu} 239(n, f) F P$ & 1 & - & $\mathrm{T} *$ & \\
\hline $\mathrm{U} 235 \mathrm{~F}$ & $\mathrm{U} 235(\mathrm{n}, \mathrm{f}) \mathrm{FP}$ & 1 & - & $\mathrm{T} *$ & $0.01 \mathrm{MeV}$ \\
\hline NP237F & $\operatorname{Np} 237(n, f) F P$ & 1 & - & $\mathrm{T} *$ & 0.5 \\
\hline IN115N & $\operatorname{In} 115(n, n) \operatorname{In} 115 M$ & $4.268 E-5$ & $4.51 \mathrm{~h}$ & $\mathrm{~T} \dagger$ & 1.0 \\
\hline $\mathrm{U} 238 \mathrm{~F}$ & $\mathrm{U} 238(\mathrm{n}, \mathrm{f}) \mathrm{FP}$ & 1 & - & $\mathrm{T} *$ & 1.45 \\
\hline FE54P & $\operatorname{Fe} 54(n, p) M n 54$ & $2.559 \mathrm{E}-8$ & $314 d$ & $\mathrm{~T}+$ & 2.2 \\
\hline $\mathrm{NI58P}$ & $\operatorname{Ni58(n,p)Co58}$ & $1.119 \mathrm{E}-7$ & $71.7 \mathrm{~d}$ & $\mathrm{~T}+$ & 2.9 \\
\hline S32P & $\mathrm{S} 32(\mathrm{n}, \mathrm{p}) \mathrm{P} 32$ & $5.567 \mathrm{E}-7$ & $14.4 \mathrm{~d}$ & $\mathrm{~T}$ & 2.9 \\
\hline AL27P & $\operatorname{Al} 27(n, p) \operatorname{Mg} 27$ & $1.217 \mathrm{E}-3$ & $9.49 \mathrm{~m}$ & $\mathrm{~T}$ & 3.3 \\
\hline MG24P & $\operatorname{Mg} 24(n, p) \mathrm{Na} 24$ & $1.284 \mathrm{E}-5$ & $15.0 \mathrm{~h}$ & $\mathrm{~T}$ & 6.3 \\
\hline FE56P & $\operatorname{Fe} 56(n, p) M n 56$ & $7.463 E-5$ & $2.58 \mathrm{~h}$ & $\mathrm{~T}+$ & 7.5 \\
\hline AL27A & $\operatorname{Al} 27(n, \alpha) \mathrm{Na} 24$ & $1.286 E-5$ & $15.0 \mathrm{~h}$ & $\mathrm{~T}$ & 8.7 \\
\hline ZR902 & $\operatorname{Zr} 90(n, 2 n) \operatorname{Zr} 89$ & $2.456 E-6$ & $3.27 \mathrm{~d}$ & $\mathrm{~T}$ & 14.0 \\
\hline $\begin{array}{l}\text { For lan } \\
\text { fission }\end{array}$ & $\begin{array}{l}\text { hanum } \\
\text { product }\end{array}$ & $6.272-7$ & $12.79 \mathrm{~d}$ & & \\
\hline
\end{tabular}

Th Thermal -- Irradiate bare and cadmium-covered. Subtract cadmium-covered activity from bare activity to get thermal response.

$R$ Intermediate - Energy listed is that of principal resonance. 
T Threshold -- Energy listed is threshold energy.

+ Place in cadmium covers to eliminate thermal interference.

* Place in boron carbide sphere. Pu239 and U235 then act like threshold detectors.

$\dagger$ Metastable state.

In a typical experiment, foils with short-lived activities are removed from the fixture as soon as possible (generally one-half hour) and transferred to the Radiation Dosimetry Laboratory for analysis. The longer-lived activities are analyzed later. In most cases, we try to obtain approximately 10,000 counts in the decay gamma-ray peak for each reaction on at least two independent detectors. Lower accumulations must sometimes be tolerated for some weakly exposed foils. 


\section{CHAPTER III}

\section{FOIL ACTIVITY MEASUREMENTS}

All of the neutron energy-integrated fluence and energy spectrum measurements described in this report rely on reaction rate measurements performed by the Radiation Dosimetry Laboratory in SNLA's Technical Area $V$. This is accomplished by the exposure of activation foils to a neutron field, followed by the nuclear counting of the foils to determine the activity of the reaction products. These measurements fall into three general classes, depending on the type of reaction product: (1) beta emitters, (2) gamma-ray emitters, and (3) fission products. The third class, fission products, are in fact gamma-ray emitters but require special counting procedures. They also differ from the second class of measurements in that the information obtained from them is the number of fissions that occurred in the foil, rather than product nuclide activity. They are therefore considered as a separate category. A list of foils and their reaction products has been given previously in Table II.1.

\section{A. Beta Counting System}

Beta counting is used to determine $32_{\mathrm{P}}$ activities resulting from the $(n, p)$ reaction in $32 \mathrm{~S}$. The beta counting system consists of an automatic sample changer and a shielded $2 \pi$ gas-flow proportional detector. The counting gas is ultrahigh-purity P10 (10\% methane in argon). A "guard" detector operates in anticoincidence with the beta detector to eliminate cosmic events for low-background counting. The detector has been fitted with a $0.254-\mathrm{mm}$ (0.010-inch) thick aluminum window. Thus, the actual counting geometry is somewhat less than $2 \pi$. Data storage and analysis, as well as control of the counting system, are accomplished with a digital computer interfaced to the beta counting system. The system is capable of very high count rates $(1 E+6$ counts/min) without excessive system dead time.

Sulfur "foils" used by the Radiation Dosimetry Laboratory are in the form of pressed, high-purity, natural sulfur pellets with a thin zein coating that prevents contamination and reduces chipping.[13] This coating is a vegetable protein that has no effect on either the radiation response of the pellet or the beta counting. The pellets have a diameter of $6.35 \mathrm{~mm}(0.25 \mathrm{inch})$, a thickness of $3.61 \mathrm{~mm}(0.142 \mathrm{inch})$, and a mass of $0.217 \pm 1 \% \mathrm{~g}$. This thickness is sufficient for the pellet to be considered infinitely thick for the $1710-\mathrm{keV}$ betas. Thus, small variations in thickness have no effect on the neutron sensitivity of a pellet. sulfur pellets are counted intact, without chemical separation or burning to extract the $32_{\mathrm{P}}$. Pellets are not reused. 
Natural sulfur is composed of ${ }^{32} \mathrm{~S}(95 \%), 34 \mathrm{~S}(4.22 \%)$, and trace amounts of other isotopes. The presence of ${ }^{34} \mathrm{~S}$ leads to several competing reactions that can interfere with the counting of the $1710-\mathrm{keV}$ betas. The first of these is the ${ }^{34} \mathrm{~S}(n, r){ }^{35} \mathrm{~S}$ reaction with thermal neutrons. The product is comparatively long-lived $(86$ d) and decays by emission of a $0.167-\mathrm{keV}$ beta. The $0.254-\mathrm{mm}$ aluminum window on the detector effectively eliminates this low-energy beta. The second reaction of importance is the $34 \mathrm{~S}(\mathrm{n}, \alpha){ }^{31} \mathrm{Si}$ reaction with fast $(>4.5-\mathrm{MeV})$ neutrons. This reaction product is comparatively short-lived $(2.6 \mathrm{~h})$ but decays by emission of a 1491-kev beta that is not removed by the aluminum window. Rather, the pellets are allowed to decay for at least $10 \mathrm{~h}$ to reduce the short-lived activity to a level that is no longer significant.

Determination of neutron fluence or pellet activity includes adjustment of the measured data for the decay of $32_{P}$ from the time of the irradiation until the pellets are counted. Corrections are also made for detector background (typically 1 to 3 counts per minute) and for counting system dead time, which depends on count rate and system electronics. Data are reported as specific activity at the time of the irradiation, or fluence $>3 \mathrm{MeV}$ if the spectrum-averaged cross section for the particular neutron field is known. In the case of an extended irradiation, the data are adjusted to the end of the irradiation.

Calibration of the beta counter is accomplished by irradiating sulfur pellets to a known fluence (and spectrum) of neutrons from a $252 \mathrm{Cf}$ spontaneous fission source and counting the resulting beta particles. The efficiency $\varepsilon$ of the detector system is determined from

$$
\varepsilon=\frac{C f_{\tau} \exp \left(\lambda t_{d}\right) \lambda t_{i}}{N \bar{\sigma} \Phi\left[1-\exp \left(-\lambda t_{c}\right)\right]\left[1-\exp \left(-\lambda t_{i}\right)\right]}
$$

where

$$
\begin{aligned}
C & =\text { counts recorded in detector, less background } \\
\mathrm{E}_{\tau} & =\text { correction for detector dead time } \\
\lambda & =32 \mathrm{P} \text { decay constant, } \mathrm{s}^{-1} \\
t_{\mathrm{d}} & =\text { decay time, } \mathrm{s} \\
t_{c} & =\text { count time, } \mathrm{s} \\
t_{i} & =\text { duration of irradiation, s } \\
\mathrm{N} & =\text { number of } 32 \mathrm{~s} \text { atoms in pellet }
\end{aligned}
$$




$$
\begin{aligned}
& \bar{\sigma}=\text { spectrum-averaged cross section for }{ }^{32} \mathrm{~S}, \mathrm{~cm}^{2} \\
& \Phi=\text { neutron fluence, } \mathrm{n} / \mathrm{cm}^{2} .
\end{aligned}
$$

The dead-time correction, $f_{\tau}$, is a polynomial of the form

$$
f_{\tau}=a_{0}+a_{1} R+a_{2} R^{2}
$$

where $R$ is the measured count rate, and the $a_{i}$ are empirically determined coefficients.

Once the system efficiency is known, foil activities can be determined for pellets irradiated in an undetermined neutron field:

$$
A_{0}=\frac{C f_{\tau} \exp \left(\lambda t_{d}\right) \lambda}{\varepsilon\left[1-\exp \left(-\lambda t_{c}\right)\right]}
$$

where $A_{0}$ is the pellet activity adjusted to the end of the irradiation.

This calibration is specific for a given pellet form and counting geometry, but it is valid for any arbitrary spectrum. Determination of the neutron fluence, however, requires knowledge of the spectrum-averaged cross section for the specific neutron field. The neutron fluence is found from

$$
\Phi=\frac{A_{0} \lambda t_{i}}{N \bar{\sigma}\left[1-\exp \left(-\lambda t_{i}\right)\right]}
$$

for an extended irradiation, and

$$
\Phi=\frac{{ }^{\mathrm{A}_{0}}}{\mathrm{~N} \bar{\sigma} \lambda}
$$

for a pulse.

This system is capable of measuring neutron fluences from approximately $5 E+9$ to $1 E+14$ neutrons $/ \mathrm{cm}^{2}$, with an estimated overall counting error of 5 to $10 \%$. Sources of error include $252 \mathrm{Cf}$ calibration, $3 \%$; sample positioning, $0.5 \%$; counting statistics, $0.1-10 \% ;$ and spectrum-averaged cross section, $3 \%$ The lower limit in sensitivity is determined by the 
detector background, while the upper limit is determined by the validity of the dead-time correction for the counting system.

\section{B. Gamma-Ray Spectrometer System}

Most of the activation foils counted by the Radiation Dosimetry Laboratory decay by beta emission, but they do so in coincidence with one or more gamma rays. These gamma rays can be counted with high-resolution detectors, such as Ge(Li) or hyperpure germanium, to determine the foil activities.

The activation analysis system in use by the Radiation Dosimetry Laboratory consists of several high-resolution detectors connected to a Canberra series 90 MCA system. Each detector is shielded by a lead housing with $10-\mathrm{cm}$ (4-inch) thick walls. A $1.27-\mathrm{cm}(0.5-i n c h)$ thick steel liner prevents lead $x$-rays from entering the detector from the shield walls. Two of the detectors have $15-\mathrm{cm}$ (6-inch) thick low-background steel shields equipped with computer-controlled sample changers.

Data are collected as 4096- or 8192-channel spectra, with peaks in the spectra corresponding to the decay gamma rays from the foil. These spectra are transmitted from the MCA via a high-speed data link to a vax-8200 computer for storage and analysis. Control and data analysis are performed by the VAX computer, using Canberra-supplied APOGEE analysis software.[14] This software package incorporates the methods of SAMPO 80 and includes routines for energy, peak shape, and efficiency calibrations, peak identification, area analysis, nuclide identification, and quantitative analysis.[15] $\mathrm{Nu}-$ clide activities are determined by a linear least-squares analysis of peak data, with nuclides selected from an appropriate library.

After a foil is cleaned and weighed, it is placed near the face of a detector in one of several standard counting geometries. The most commonly used geometries are at distances of 5 and $15 \mathrm{~cm}$, with the inclusion of a $0.95-\mathrm{cm}$ (0.375-inch) thick aluminum filter between the foil and the detector. These distances insure that coincident summing errors are small ( $<1 \%)$ for any of the nuclides of interest. Coincidence summing errors resulting from any beta particles from the foil are eliminated by the aluminum filter.

A foil is normally placed at the closer $(5 \mathrm{~cm})$ geometry unless high counting rates require a greater distance. The MCA system automatically adjusts the actual counting time for the time lost during an ADC analysis cycle ("dead time"). However, saturation effects in the detector cannot be corrected for in this manner, and the total count rate is generally limited to approximately 3000 counts per second. This 
corresponds to a dead time of about $2 \%$, although dead times up to about $20 \%$ can be tolerated without seriously affecting the measurements.

Results are reported as reaction product activity in becquerels per gram $(\mathrm{Bq} / \mathrm{g})$ of target isotope. Overall counting errors are reported at a $90 \%$ confidence level and normally range from 3 to $7 \%$. Sources of error include calibration source errors, $0.9-4.0 \%$; sample positioning, $0.5 \%$; and counting statistics, $0.5-5 \%$.

The different foils used by the Radiation Dosimetry Laboratory have been listed in Table II.1. Data relevant to the nuclear counting of these foils are given in Table III.1.

Calibration of the gamma-ray spectrometer is accomplished in several steps and involves energy, peak shape, and efficiency calibrations for each detector and geometry. An Amersham QCD.1 mixed radionuclide standard is placed in one of the standard counting geometries.[16] This standard is an elevenline gamma-ray source totalling approximately $1 \mu \mathrm{Ci}$. A spectrum is collected for a time sufficient to accumulate a statistically significant number of counts in each of the peaks of interest, typically at least 10,000. The spectral data are then transferred to the computer for storage and analysis.

The ability of the system to determine peak areas, and thus foil activities and detector efficiency, depends on the scheme used to identify a peak and determine the net counts within the peak. The APOGEE software package fits a Gaussian curve to the central portion of a peak and a separate exponential tail to each side. Peak shape parameters are determined for each of the peaks used in the calibration, and energy and shape calibrations for the detector are obtained by a linear least-squares fit to these parameters. Energy as a function of channel number is expressed as a third-degree polynomial. Second-degree polynomials are used for full-wave, half-magnitude (FWHM) and peak tailing as a function of energy. The coefficients for each of these fits are stored in an energy-calibration file for the specific detector.

The photopeak efficiency of a detector system must be determined for each geometry used with a given detector, where photopeak efficiency is defined as the number of photons collected completely by the detector divided by the number emitted by a source. Detection efficiency is dependent on gamma-ray interaction probabilities in the detector and on attenuation of the gamma rays by materials between the sample and the active region of the detector. In the absence of additional filtering material, Ge(Li) or HPGe detectors generally exhibit a maximum efficiency between 100 and $200 \mathrm{keV}$. Efficiency curves are typically divided into two segments, separated by a "crossover" energy in the vicinity of 
Table III. 1

Foil Nuclear Counting Parameters

\begin{tabular}{|c|c|c|c|}
\hline Nuclide & $\begin{array}{l}\text { Energy } \\
(\mathrm{keV})\end{array}$ & $\begin{array}{l}\text { Yield } \\
\left(\frac{\circ}{0}\right)\end{array}$ & $\begin{array}{l}\text { Half Life } \\
\text { (min) }\end{array}$ \\
\hline $\mathrm{Au}-198$ & $\begin{array}{r}411.8044 \\
675.8875 \\
1087.6631\end{array}$ & $\begin{array}{r}95.51 \\
1.06 \\
0.23\end{array}$ & 3882.24 \\
\hline $\mathrm{Ba}-140$ & $\begin{array}{l}162.6400 \\
304.8400 \\
423.7000 \\
437.5500 \\
537.3200\end{array}$ & $\begin{array}{r}6.70 \\
4.50 \\
3.20 \\
2.00 \\
25.00\end{array}$ & 18416.2 \\
\hline $\mathrm{Ce}-141$ & 145.4400 & 48.40 & 46800.0 \\
\hline Co- 58 & $\begin{array}{r}810.7570 \\
863.9351 \\
1674.6799\end{array}$ & $\begin{array}{r}99.40 \\
0.74 \\
0.54\end{array}$ & 101952 . \\
\hline $\mathrm{Co}-60$ & $\begin{array}{l}1173.2158 \\
1332.4860\end{array}$ & $\begin{array}{l}99.86 \\
99.98\end{array}$ & $2.772335 E+06$ \\
\hline $\mathrm{Cu}-64$ & 1345.9000 & 0.49 & 762.060 \\
\hline $\mathrm{Fe}-59$ & $\begin{array}{r}142.6480 \\
192.3440 \\
1099.2240 \\
1291.5601\end{array}$ & $\begin{array}{r}1.03 \\
3.11 \\
56.50 \\
43.20\end{array}$ & 64267.2 \\
\hline In $-115 M$ & 336.3010 & 46.70 & 261.600 \\
\hline In $-116 \mathrm{M}$ & $\begin{array}{l}1097.2100 \\
1293.5400\end{array}$ & $\begin{array}{l}55.30 \\
84.50\end{array}$ & 54.1500 \\
\hline
\end{tabular}


Table III.1 (Continued)

Foil Nuclear Counting Parameters

\begin{tabular}{|c|c|c|c|}
\hline Nuclide & $\begin{array}{l}\text { Energy } \\
(\mathrm{keV})\end{array}$ & $\begin{array}{l}\text { Yield } \\
\left(\frac{o}{\partial}\right)\end{array}$ & $\begin{array}{l}\text { Half Life } \\
\text { (min) }\end{array}$ \\
\hline $\mathrm{La}-140$ & $\begin{array}{r}328.7680 \\
487.0290 \\
815.8500 \\
1596.4900\end{array}$ & $\begin{array}{l}20.50 \\
45.50 \\
23.50 \\
95.49\end{array}$ & 2413.20 \\
\hline$M g-27$ & 843.7600 & 71.80 & 9.45800 \\
\hline$M n-54$ & 834.8270 & 99.97 & 450288 \\
\hline$M n-56$ & $\begin{array}{r}846.7520 \\
1810.6899\end{array}$ & $\begin{array}{l}98.90 \\
27.20\end{array}$ & 154.710 \\
\hline $\mathrm{Na}-24$ & 1368.5300 & 100.00 & 900.000 \\
\hline Mo-99 & $\begin{array}{l}140.5080 \\
181.0630 \\
366.4300 \\
739.5800 \\
778.0000\end{array}$ & $\begin{array}{r}3.80 \\
6.20 \\
1.37 \\
12.80 \\
4.50\end{array}$ & 3961.20 \\
\hline $\mathrm{Zr}-89$ & 909.1000 & 99.04 & 4705.80 \\
\hline $\mathrm{Zr}-95$ & $\begin{array}{l}724.1840 \\
756.7150\end{array}$ & $\begin{array}{l}43.70 \\
55.30\end{array}$ & 92188.8 \\
\hline
\end{tabular}

150 to $300 \mathrm{keV}$. Above this crossover energy, log(efficiency) is a nearly linear function of log(energy). Below the crossover energy, this relationship is approximately parabolic. given by

At a given photon energy, the detection efficiency is

$$
\varepsilon(E)=\frac{C \exp \left(\lambda t_{d}\right)}{t_{C^{Y}{ }^{A}}^{A}}
$$

where

$$
\varepsilon(E)=\text { detection efficiency at photopeak energy } E
$$




$$
\begin{aligned}
C= & \text { net counts in photopeak at energy } E \\
\lambda= & \text { decay constant for calibration nuclide, } \mathrm{s}^{-1} \\
\mathrm{~A}= & \text { nuclide activity at source reference time, } \mathrm{Bq} / \mathrm{g} \\
\mathrm{Y}_{\gamma}= & \text { absolute yield of photons at energy } \mathrm{E}, \text { photons per } \\
& \text { disintegration } \\
t_{\mathrm{d}=}= & \text { decay time of calibration source, i.e., elapsed } \\
& \text { time from source reference time until count is } \\
& \text { started, s } \\
t_{c}= & \text { collect "live" time, s. }
\end{aligned}
$$

The detection efficiency is determined for each of the peak energies, spanning the entire range over which subsequent measurements will be made. Two sets of calibration coefficients are determined, based on a least-squares fit weighted with uncertainties in peak areas and calibration source activities. A second-degree polynomial is used below the crossover energy, while a third-degree polynomial is used above. The coefficients are stored in the detector calibration file, along with their variances and covariances. A typical detector efficiency curve is shown in Figure III.1. The circles represent measured detector efficiency, while the solid line is the fit to the data.

\section{Fission Foil Counting}

The Radiation Dosimetry Laboratory uses fission foils in several forms, the most common of which is encapsulated pellets of oxides of $238 \mathrm{U}, 235 \mathrm{U}, 239 \mathrm{Pu}$ or $237 \mathrm{~Np}$, usually enclosed in cadmium covers and a boron carbide sphere, as described in Chapter II. Also used is $235 \mathrm{U}$ metal foil, sometimes alloyed with aluminum or zirconium to reduce sensitivity or increase temperature range.

After irradiation, a fission foil is counted with a high-resolution gamma-ray spectrometer, as described in the previous section. The specific isotopes of interest are fission products, primarily the 140 La fission-product chain:

$$
\begin{aligned}
& { }^{140} \mathrm{I} \longrightarrow{ }^{140} \mathrm{Xe} \longrightarrow{ }^{140} \mathrm{Cs} \rightarrow{ }^{140} \mathrm{Ba} \longrightarrow{ }^{140} \mathrm{La} \longrightarrow{ }^{140} \mathrm{Ce} \\
& (0.8 \mathrm{~s}) \quad(14 \mathrm{~s}) \quad(65 \mathrm{~s})(12.79 \mathrm{~d})(40.3 \mathrm{~h}) \text {. }
\end{aligned}
$$

This fission-product chain is particularly useful because the fission yield is relatively high $\left(\simeq 6 \frac{6}{6}\right)$, it produces a highenergy gamma ray at $1596 \mathrm{keV}$ that is normally free from other 


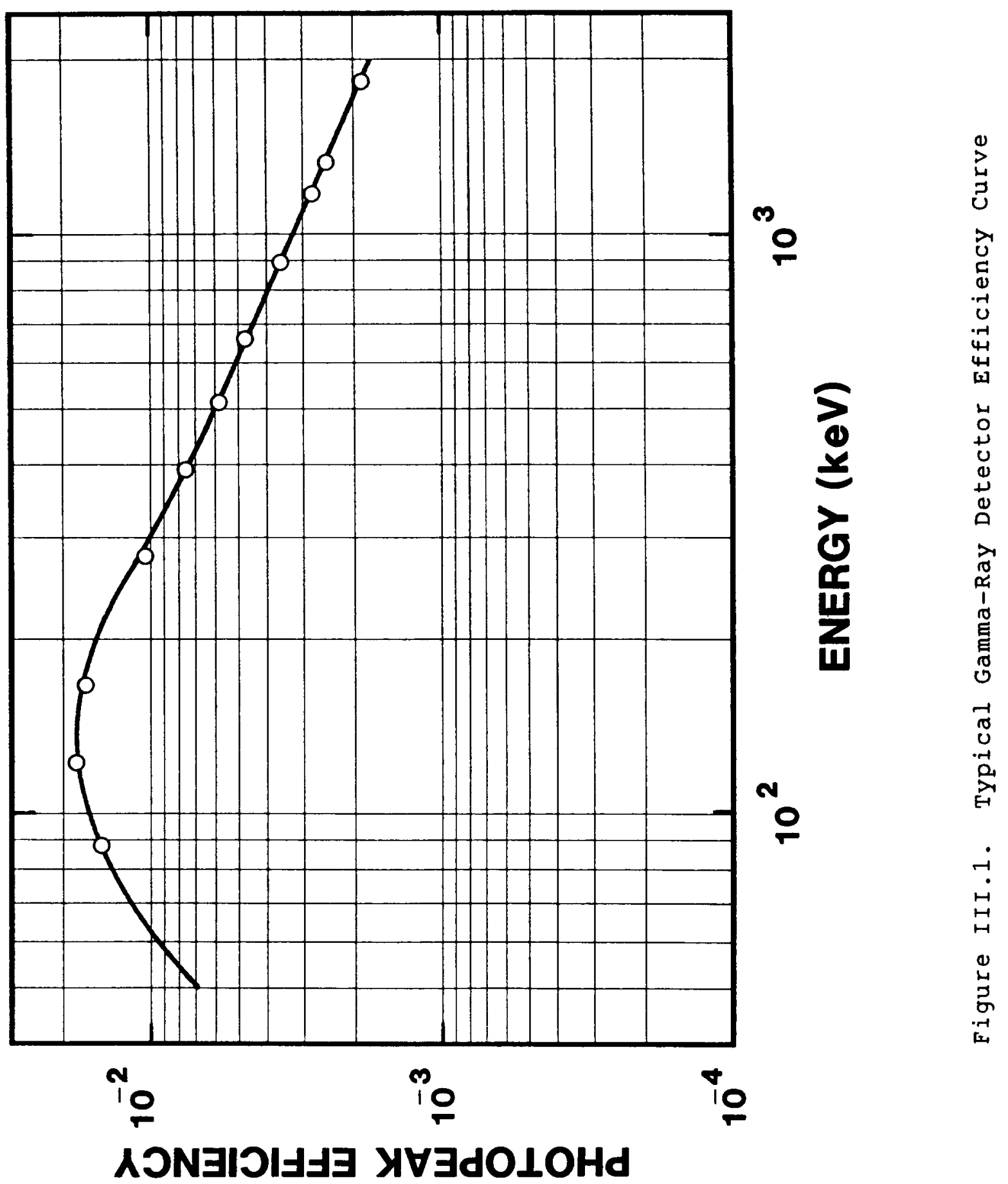


interfering gamma-ray lines, and the dominant $140_{\mathrm{Ba}}$ half-life of 12.79 days is convenient for nuclear counting.

Many fission products produced in the foils emit low-energy photons. These isotopes complicate the nuclear counting because their half-lives are short, and hence their activities are high. Under some circumstances, the 1596-kev gamma ray from 140 La can be completely masked by excessively high detector dead time. Analysis times are also increased significantly. To avoid this, a $1.27-\mathrm{cm}(0.5-i n c h)$ lead filter is placed between the foil and the detector. This filter eliminates the bulk of low-energy photons while reducing the $1596-\mathrm{keV}$ line by about a factor of two. To further reduce the number of peaks in the resulting spectrum that must be analyzed, the lower-level discriminator on the MCA system is raised.

Procedures for calibrating the detector for use with fission foils are similar to those for other activation foils. However, the lead filter considerably alters the shape of the efficiency curve. The curve exhibits a broad maximum between 500 and $1500 \mathrm{keV}$, and a two-part efficiency curve is no longer appropriate. Therefore, a single polynomial is used for the energy range 300 to $2000 \mathrm{~V}$. This is accomplished in the APOGEE software by specifying a crossover energy of zero, rather than the 150 to $300-k e v$ crossover energy described in the previous section. A typical detector efficiency curve for the lead configuration is shown in Figure III.2.

As illustrated above, the fission products of interest to the Radiation Dosimetry Laboratory are contained in fissionproduct decay chains. As a result, corrections for decay time depend on the individual isotope decays as well as the individual fission yield for the several isotopes in the chain. In fact, $140 \mathrm{La}$ activity undergoes a buildup due to the decay of its $140 \mathrm{Ba}$ precursor, as illustrated in Figure III.3. APOGEE software is used to obtain ${ }^{140}$ La activity, uncorrected for decay. This activity is then used to obtain the number of fissions produced in the foil, according to the equation

$$
F=A_{L a} / C_{d}
$$

where

$$
\begin{aligned}
& F=\text { fissions per gram in foil } \\
& \mathrm{A}_{\mathrm{La}}=\text { activity of } 140 \mathrm{La} \text { at time of counting, } \mathrm{Bq} / \mathrm{g} \\
& \mathrm{C}_{\mathrm{d}}=\text { correction for buildup and decay of } 140 \mathrm{La} \text {, }
\end{aligned}
$$




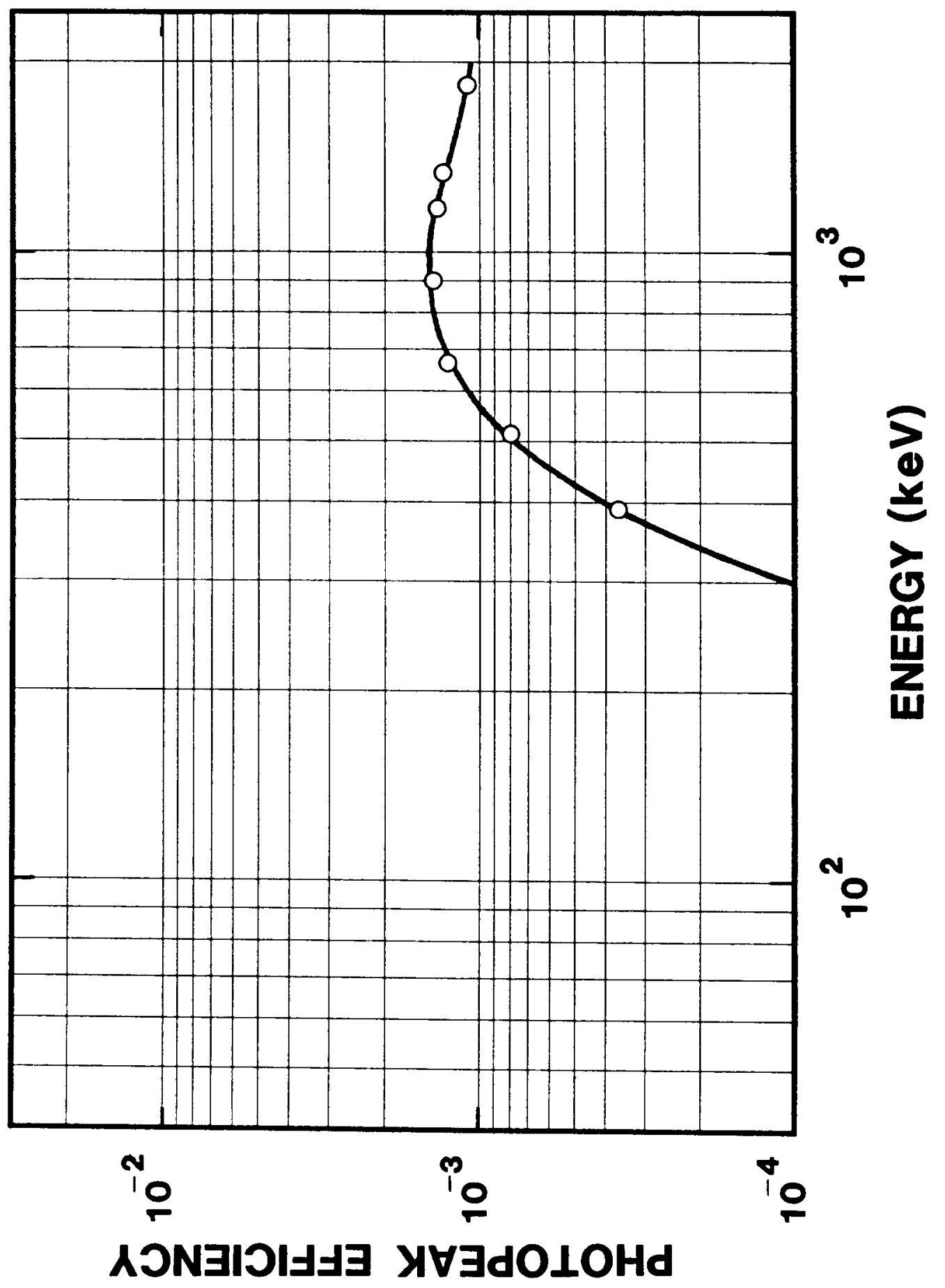

$\stackrel{0}{3}$

Ư

5
0
01
0
0
0
0
0
-14
4
0
0

0
0
0

年

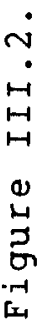




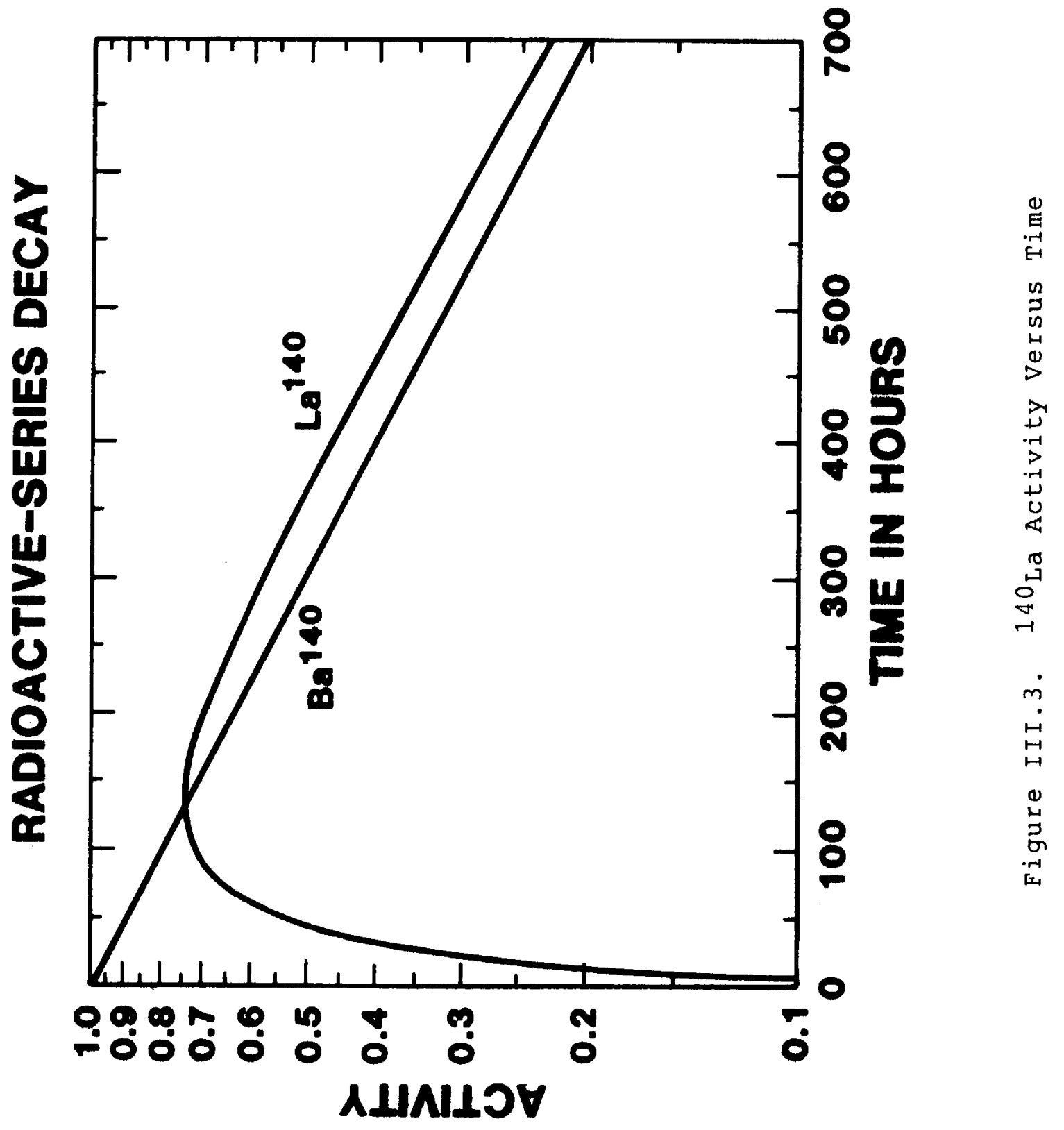




$$
\begin{aligned}
C_{d} & =\frac{Y_{B a} / 100}{t_{C}}\left\{\frac{\lambda_{L a}}{\lambda_{L a}-\lambda_{B a}} \exp \left(-\lambda_{B a} t_{d}\right)\left[1-\exp \left(-\lambda_{B a} t_{C}\right)\right]\right. \\
& \left.-\left(\frac{\lambda_{B a}}{\lambda_{L a}-\lambda_{B a}}-\frac{Y_{L a}}{Y_{B a}}\right) \exp \left(-\lambda_{L a} t_{d}\right)\left[1-\exp \left(-\lambda_{L a} t_{C}\right)\right]\right\}
\end{aligned}
$$

where

$$
\begin{aligned}
\lambda_{\mathrm{Ba}} & =140_{\mathrm{Ba}} \text { decay constant, } \mathrm{s}^{-1} \\
\lambda_{\mathrm{La}} & =140_{\mathrm{La}} \text { decay constant, } \mathrm{s}^{-1} \\
\mathrm{Y}_{\mathrm{Ba}} & =\text { cumulative fission yield of } 140_{\mathrm{Ba}}, \text { percent } \\
\mathrm{Y}_{\mathrm{La}} & =\text { independent fission yield of } 140_{\mathrm{La}} \text {, percent } \\
t_{\mathrm{d}} & =\text { decay time, } \mathrm{s} \\
t_{\mathrm{C}} & =\text { count time, } \mathrm{s} .
\end{aligned}
$$

The yields of the various fission products depend on the energy of the neutron inducing the fission. However, since fission foils are generally used inside boron carbide spheres, a fast reactor spectrum is appropriate even in the ACRR. Fission yields for various fission products were obtained from Reference 16 and are listed in Table III.2.

Results are reported as reaction product activity in fissions per gram of target isotope. Overall counting errors are reported at a $90 \%$ confidence level, and normally range from 4 to $8 \%$. Sources of error include calibration source errors, $1.0-5.0 \%$; sample positioning, $3.0 \%$; and counting statistics, $0.5-5 \%$. 


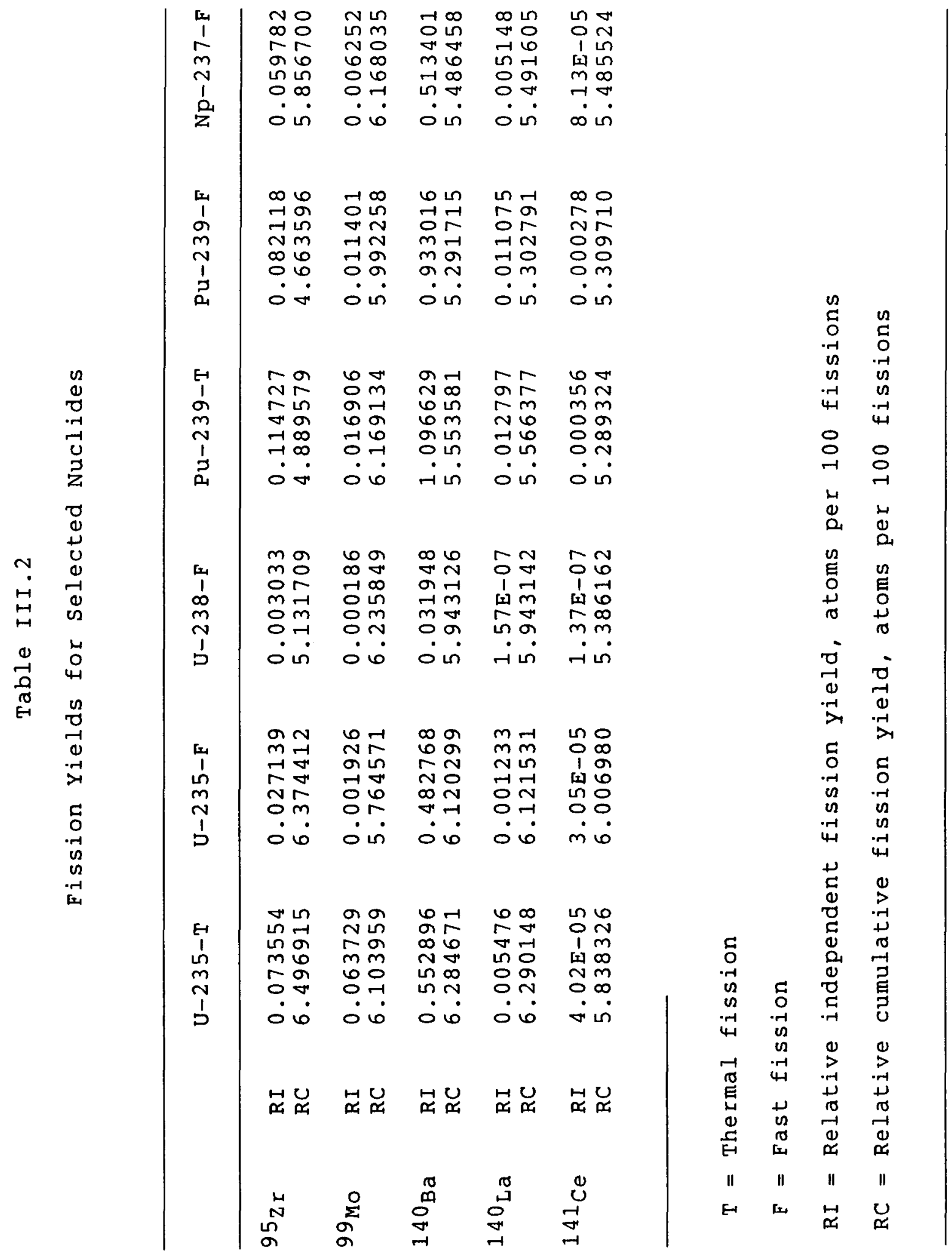


Although the neutron spectra generated in the SPR III and the ACRR have been measured in the past[17]--by more than one technique in some cases[18]--incentives have developed that justify additional work. These include

- The additional testing of electronic components in new environments

- The surfacing of some laboratory and interlaboratory discrepancies

- The acquisition of more accurate and complete cross-section sets

- Recent requests for reactor characterization of a much broader range of environments

The last incentive has been the primary driver for our seeking adjustment techniques that exhibit less dependence on the code being used or on the trial spectrum that initiates the adjustment procedure. Fundamental, 3D calculations that require advanced codes such as MORSE[10] are lengthy and expensive to run, and it is not practical to use these codes to calculate every spectrum that should be used by experimenters. Furthermore, accurately calculated spectra should be verified in a few "benchmark" cases before the calculations in other geometries can be relied upon.

The method we have used is to increase the analyst's interaction with the adjustment procedure by transporting SANDII to the Digital Equipment VAX-8650 so that the code can be used interactively and run many times while a spectrum is being sought. (Another objective has been to compare the SANDII results with those of other codes applied to the same data. These comparisons have not yet been carried out.) It has been known for many years that SANDII cannot iterate directly to an acceptable solution spectrum that is compatible with the measured foil activities from arbitrary trial functions. That is the reason for incorporating a library of trial functions in the code or for relying on calculated trials. Our concern was that such trials may automatically bias the result or miss important features. Certainly, if all adjusted spectra begin with the same trial function, at least their ultimate differences will depend only on the fundamental differences among the data sets.

Although the different codes use a variety of algorithms that allow the code to arrive at a solution and may have different ways to incorporate additional constraints (such as 
minimizing curvature in the final spectrum or requiring positive values everywhere), they must all in the end compare measured and calculated activities. These calculated activities are obtained from the integral in Equation IV.1.

$$
A=N \lambda_{i} \int \sigma_{i}(E)_{i} \phi(E) d E
$$

where

$$
\begin{aligned}
A= & \text { total activity of foil } \\
N= & \text { number of atoms of } i \text { th isotope in foils } \\
\sigma_{i}(E)= & \begin{array}{l}
\text { reaction cross section versus energy of foil } \\
\text { material } i
\end{array} \\
\phi(E)= & \text { differential fluence value at energy } E, \text { where } \\
& \Phi(E), \text { total fluence above } E, \text { is equal to } \int_{E}^{\infty} \phi(E) d E \\
\lambda_{i}= & \text { decay constant for reaction, } s^{-1} .
\end{aligned}
$$

Therefore, if the spectrum $\phi(E)$ with which the code begins is the "right" one (compatible with all activities and other constraints), then all codes should acknowledge that it is the right answer, and whatever algorithm is available for changing the trial may not even be used unless other constraints are violated. Our procedure has been to find a solution that is very close to being compatible from the start and also to investigate whether other solutions are equally acceptable. The investigations thus far have shown that, for a good foil set, other solutions that are as acceptable usually differ by only small amounts over almost all the energy regions of interest.

One characteristic of SANDII that we have found extremely useful is that the code will supply clues, sometimes powerful and simple, sometimes weak and subtle, as to how a better trial function might be chosen and as to whether a particular measured activity may be in error. It is helpful if the analyst is aware of the energy regions in which each foil responds to the spectrum being investigated.

When SANDII was first used in this project in 1983, we noticed that, with the internal cross sections received with the code, the sulfur and nickel threshold reactions at about $3 \mathrm{MeV}$ could not both be made compatible with any spectrum (sulfur high by $\simeq 15 \%$ ). In addition, the $\operatorname{Mn} 55(n, \gamma) \operatorname{Mn} 56$ reaction was not compatible with spectra that had low fluence 
values near the resonance energy at about 3E-4 MeV. For these cases, the response of manganese was shifted by the spectrum to much higher energies where the cross section was perhaps not so well determined. Therefore, the latest Radiation Shielding Information Center (RSIC) multigroup cross sections in SANDII format for all the reactions were obtained from oak Ridge National Laboratory (ORNL). [19] They are based on the Evaluated Nuclear Data Files ENDF/IV and ENDF/V. Since that time, a later set of cross-section data has been obtained from Los Alamos by Clarence Lee.[20] For the reactions we have used, a point-by-point comparison has shown negligible differences from the ORNL RSIC values.

The adjusted spectra obtained with the RSIC cross-section set were much smoother, and the standard deviation (SD) of the calculated to measured activities decreased dramatically. The sulfur and nickel foils agreed to a few percent. It is clear that the best cross sections must be used if acceptable adjusted spectra are to be generated.

In our working version of the cross-section library, two changes have been made. First, the reaction zr90(n,2n)zr89 with a threshold at $14 \mathrm{MeV}$ was added from the old SANDII library. Second, the boron cross section used in the cover attenuation for the fission foils in the boron ball was changed from a total cross section (in the RSIC library) to the absorption cross section (also from the older version of the code). We felt that, in geometries in which scattered neutrons are not necessarily lost from the beam (as in the reactor core center), only absorbed neutrons would be significantly attenuated by the boron ball. Certainly, more reasonable spectra were obtained with this substitution, but the change is less well justified when leakage spectra are being determined.

In this chapter, the adjustment process for two important reactor configurations will be discussed in detail so as to illustrate the steps that are used to obtain acceptable solutions. We have found that each spectrum must be treated individually, and that the analyst can use his knowledge in ways that may be difficult to incorporate into the computer program. In this regard, our methods may seem somewhat primitive and subjective. In most cases, however, the results exhibit very little dependence on the form of the initial trial function. 


\section{A. Developing a SPR III Bare Cavity spectrum.}

The first case considered here is the measurement of the spectrum in the $17-\mathrm{cm}(6.5$-inch) diameter central cavity of SPR III. This is the activity set from which most of the procedures for obtaining SANDII solutions were worked out. However, a more current data set, without the boron ball cover for the fission foils, was used to obtain the SPR III cavity spectrum presented in Chapter $V$. A simplified drawing of the reactor is shown in Figure IV.1. The majority of experiments are conducted inside a boron carbide-lined tube, called the thimble, which is designed to absorb low-energy neutrons. However, there is a large opening in the top which affects the low-energy end of the spectrum.

The foils were placed on the rings equidistant from the vertical flux centerline, as described in chapter II. In one of two runs, a boron ball with the fission foils was placed at the centerline. The reactor was operated in pulsed mode for these exposures.

The activities measured in the SPR III cavity that have been used in an adjustment operation are listed in Table IV.1. In addition, Table IV.l lists other activities, marked with an asterisk, that have not been used in the analysis. The reasons for their non-use will be discussed below. Extensive notes about the reactions follow this table.

We now trace the adjustment process as it was carried out for this case, then show how a reasonably chosen trial spectrum can lead an investigator astray if he or she is inexperienced or not alert. A trial spectrum calculated by personnel of the Nuclear Effects Laboratory at White Sands Missile Range (WSMR) was chosen.[23] The plot in Figure IV.2 shows the typical shape generally assumed for the cavity for this type of reactor. However, the spectrum values for the trial were only supplied down to $1 \mathrm{keV}$. For cavity spectra, SANDII is generally directed to attach a low-energy tail proportional to E.

When this trial was substituted into the program with the measured activities, the spectrum shown in Figure IV.3 was calculated after 23 iterations. The SD of measured-tocalculated activities achieved was $6.72 \%$

The complicated structure is caused by the cross sections for foils whose activities do not fit the trial function very well. Obviously, the small quantity of material contained in the foils cannot modify the spectrum to the extent seen here. SANDII simply tries to achieve activity agreement by modifying the trial most where each response is highest. Some of the resonance peaks of the various foils have been marked because we use these as clues for constructing a better trial. 


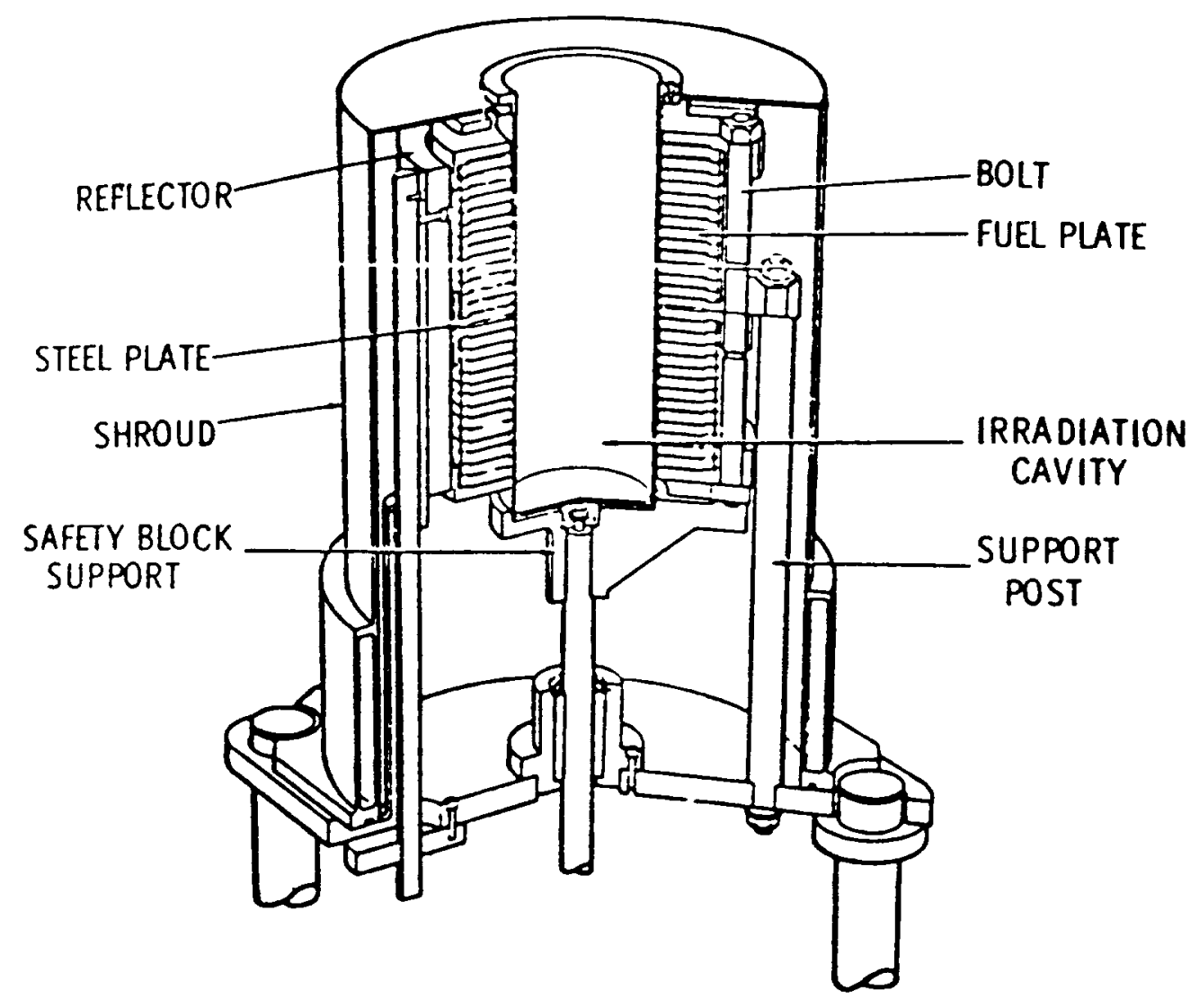

Figure IV.1. Drawing of SPR III 
Table IV. 1

Activities of Foils Exposed in SPR III Bare Cavity

\begin{tabular}{|c|c|c|}
\hline Reaction & Cover (atoms/barn) & $\begin{array}{c}\text { Activity } \\
\text { (Bq/nucleus) }\end{array}$ \\
\hline Au197(n, r)Au198 & $2.587 \mathrm{E}-3$ cadmium & $2.170 E-16$ (a) \\
\hline Au198(n, r)Au198 & - & $2.277 \mathrm{E}-16 \quad(\mathrm{a})$ \\
\hline $\operatorname{Mn} 55(n, \gamma) \operatorname{Mn} 56$ & $2.587 \mathrm{E}-3$ cadmium & $1.504 \mathrm{E}-16$ \\
\hline $\operatorname{Mg} 24(n, p) \mathrm{Na} 24$ & $2.587 \mathrm{E}-3$ cadmium & $4.092 \mathrm{E}-18$ \\
\hline $\mathrm{Fe} 56(n, p) M n 56$ & $2.587 E-3$ cadmium & $1.778 \mathrm{E}-17$ \\
\hline $\mathrm{Fe} 54(\mathrm{n}, \mathrm{p}) \mathrm{Mn} 54$ & $2.587 \mathrm{E}-3$ cadmium & $4.481 \mathrm{E}-19$ \\
\hline $\operatorname{Al27}(n, p) \operatorname{Mg} 27$ & $2.587 \mathrm{E}-3$ cadmium & $1.056 \mathrm{E}-15$ \\
\hline $\operatorname{Al} 27(n, \alpha) \mathrm{Na} 24$ & $2.587 \mathrm{E}-3$ cadmium & $1.972 \mathrm{E}-18$ \\
\hline $\operatorname{Ni} 58(n, p) \operatorname{Co} 58$ & $2.587 \mathrm{E}-3$ cadmium & $2.863 \mathrm{E}-18$ \\
\hline $\mathrm{Na} 23(\mathrm{n}, \mathrm{r}) \mathrm{Na} 24$ & - & $2.207 \mathrm{E}-18$ \\
\hline $\operatorname{Zr} 90\left(n, 2 n^{\prime}\right) \operatorname{Zr} 89$ & $2.587 \mathrm{E}-3$ cadmium & $5.874 E-20$ \\
\hline $\mathrm{S} 32(\mathrm{n}, \mathrm{p}) \mathrm{P} 32$ & - & $9.554 \mathrm{E}-18$ \\
\hline$\star \operatorname{In} 115(\mathrm{n}, \gamma) \operatorname{In} 116$ & - & $1.704 \mathrm{E}-14$ (b) \\
\hline$\star \operatorname{In} 115\left(n, n^{\prime}\right) \operatorname{In} 115 M$ & - & $2.147 \mathrm{E}-15$ (c) \\
\hline$\star \operatorname{In} 115(n, \gamma) \operatorname{In} 116$ & $2.587 \mathrm{E}-3$ cadmium & $1.698 \mathrm{E}-14$ (b) \\
\hline$\star \operatorname{In} 115\left(\mathrm{n}, \mathrm{n}^{\prime}\right) \operatorname{In} 115 \mathrm{M}$ & $2.587 \mathrm{E}-3$ cadmium & $2.185 E-15$ (c) \\
\hline$\star N i 58(n, p) \operatorname{Co} 58$ & $4.705 \mathrm{E}-3$ cadmium, 0.101 boron & $2.456 \mathrm{E}-18(\mathrm{~d})$ \\
\hline $\mathrm{U} 235(\mathrm{n}, \mathrm{f}) \mathrm{FP}$ & $4.705 E-3$ cadmium, 0.101 boron & $5.397 \mathrm{E}-10(e)$ \\
\hline$U 238(n, f) F P$ & 4.705E-3 cadmium, 0.101 boron & $7.129 \mathrm{E}-11(\mathrm{e})$ \\
\hline $\operatorname{Pu} 239(n, f) F P$ & 4.705E-3 cadmium, 0.101 boron & $7.003 \mathrm{E}-10(\mathrm{e})$ \\
\hline $\operatorname{Np} 237(n, f) F P$ & 4.705E-3 cadmium, 0.101 boron & $4.052 \mathrm{E}-10(\mathrm{e})$ \\
\hline
\end{tabular}


(a) The gold foil is a dilute solution of gold (0.155\%) in aluminum. Early experiments showed that foils of pure gold even $0.003-\mathrm{cm}$ thick suffered from self-shielding because the resonance cross section at $5 \mathrm{eV}$ is so large. The high dilution in the present foils eliminated the need for a self-shielding correction.

(b) As in the gold foil case, we verified that the $\operatorname{In} 115(n, \gamma) \operatorname{In} 116$ reaction suffered from self-shielding. The correction should be quite large (at least a factor of 3 ). Such a large correction would make this measurement very uncertain, so it has been left out of the analyses of all the spectra. The intention is to use either very thin foils or dilute ones in the future. If one wishes to attempt selfshielding corrections, the foil diameter is $1.27 \mathrm{~cm}$, and an average mass is $0.11 \mathrm{~g}$.

(c) In the spectrum measurements carried out so far, all of the activities for the $\operatorname{In} 115\left(n, n^{\prime}\right) \operatorname{In} 115 \mathrm{M}$ reaction have been too large to fit the spectra that fit the other reactions well. When this problem was discussed with Wes Sallee of WSMR, he suggested that this reaction may also be excited by the intense fluence of gamma rays impinging on the target.[21] This also suggests that encasing the foil in cadmium might make the problem worse. In any case, this reaction is not generally included in the adjustment operations.

(d) The activity of the Ni58(n,p)Co58 foil customarily placed inside the boron ball has always appeared low compared to the calculated activity. SANDII, which calculates the attenuation of the fluence in the boron ball, provided only a $3 \%$ correction to the activity. (The correction is based simply on exponential attenuation in the boron.) Experimentally for this case, however, the difference was $14 \%$. John Meason at WSMR also noticed a difference here.[22] (In his case, the measured attenuation in the ball was about $8 \%$.) We are presently preparing to calculate the attenuation with MORSE to see whether agreement with experiment can be obtained. In any case, the proper effective cross-section values for attenuation in boron are probably somewhere between the total and the absorption cross section and are determined by the geometry of the experiment.

(e) The activity of each of these four fission foils, measured in fissions per nucleus, was multiplied by 1.118 to correct for the fluence profile across the cavity diameter. 


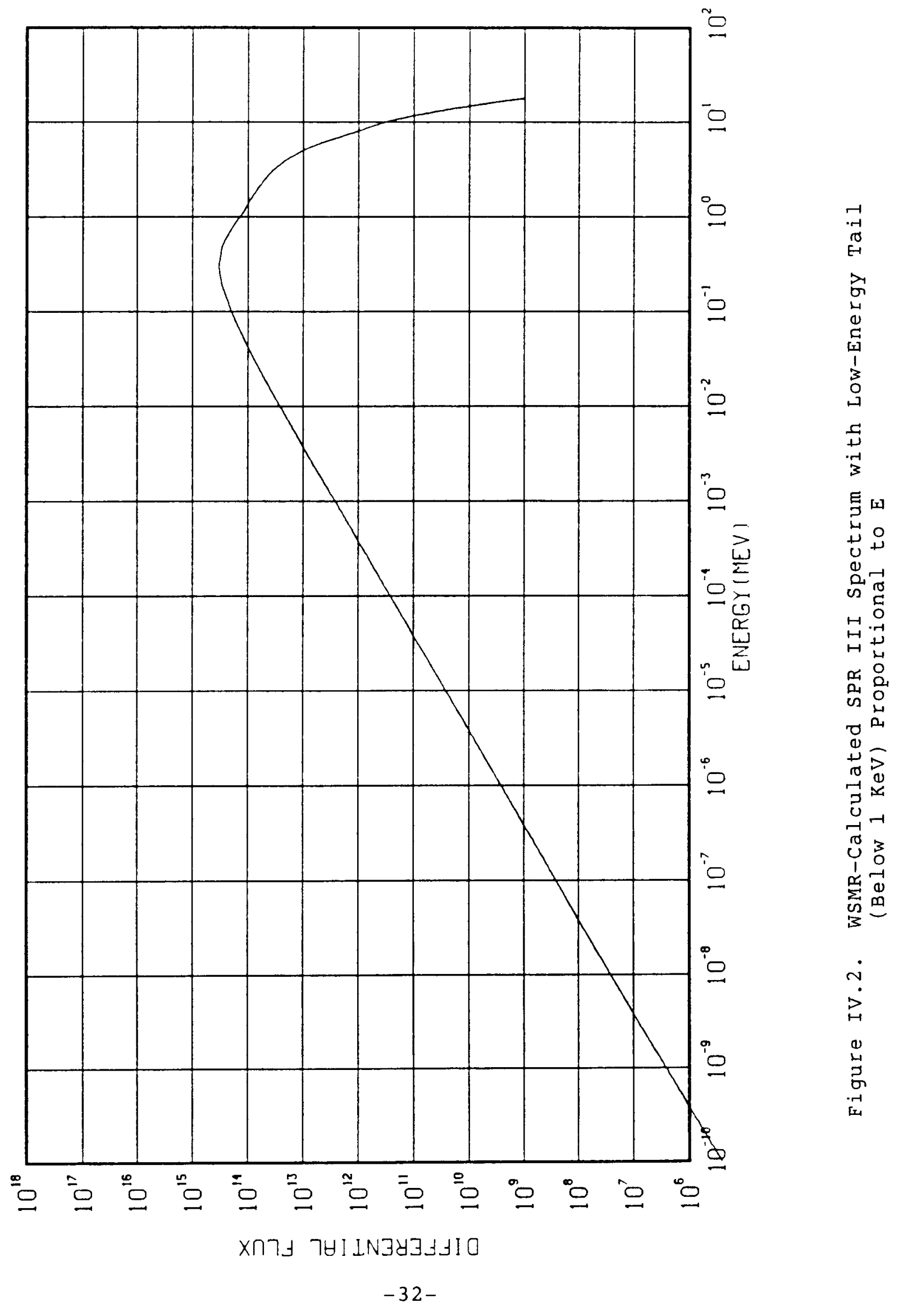




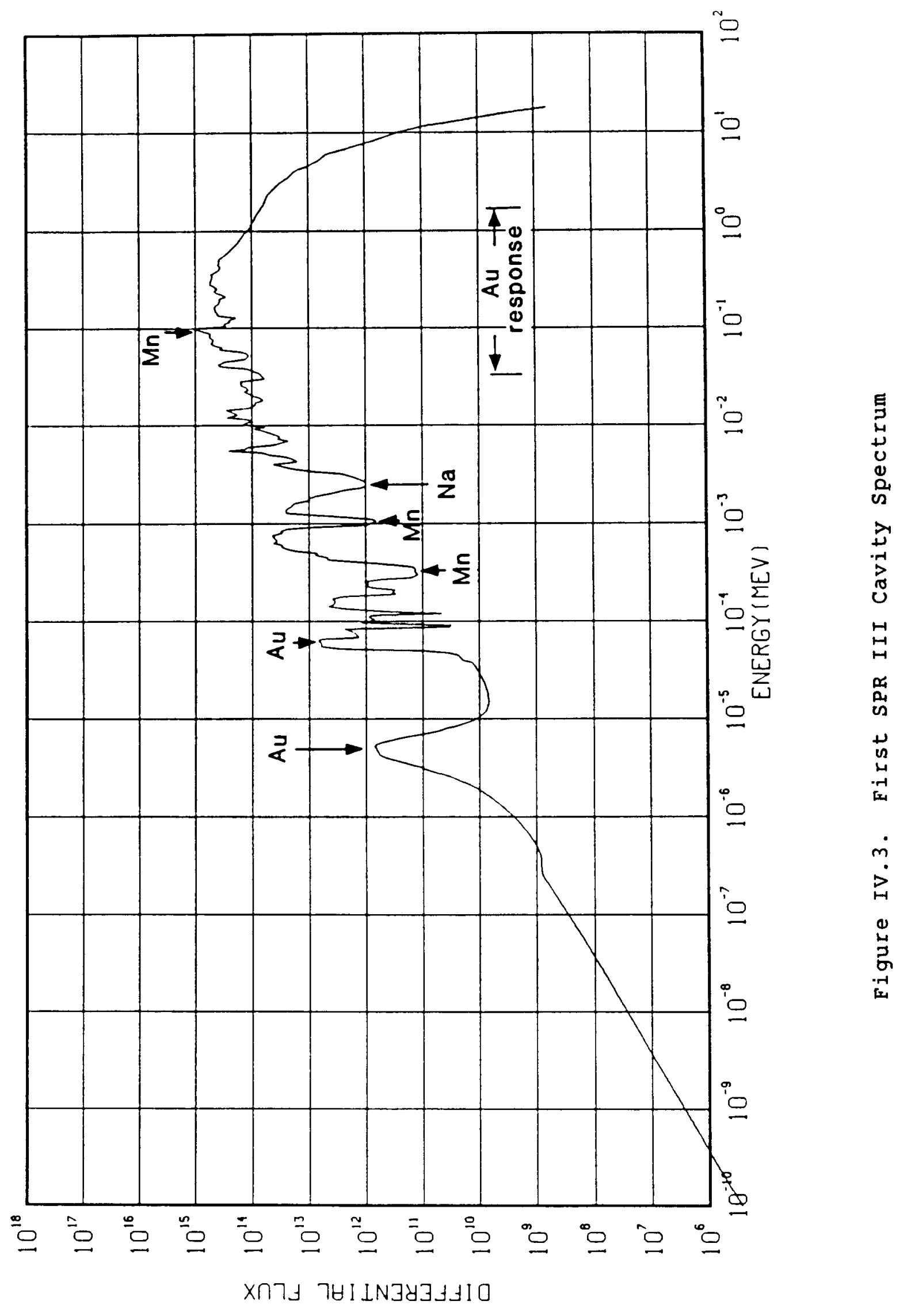


For the next trial, a smoother line is usually drawn that passes near levels where high responses of individual foils are located. SANDII also provides a printout of the energy limits between which $95 \%$ of the response of each foil lies. The gold foil is significant because, as shown in Figure IV. 3 , the bulk of the response is far above the resonance at $5 \mathrm{eV}$. The gold reaction indicates that the trial should be higher between the response boundaries, and the manganese and sodium foils indicate a location where the spectrum should be lowered. The gold resonance peak was not at first thought to be relevant for fitting because it was outside the $95 \%$ response region. Many other trial functions were tried, without success, that preserved the decreasing low-energy tail. It appeared that the gold foil activity was just not compatible with the rest and had to be discarded. Indeed, after deletion of the gold data, a smooth spectrum was obtained that, for a considerable period, we considered to be correct. The problem was that there did not seem to be any error in the gold foil data.

However, suppose we allow the result obtained with SANDII to tell us something--that there should be a thermal tail because the resonance peak is being pushed upward. Figure IV.4 shows a plot of the spectrum with a thermal tail attached at $1 \mathrm{E}-3 \mathrm{MeV}$ and with the gold data included. The fit is much better, having achieved a much-improved $3.44 \% \mathrm{SD}$. In addition, the code now indicates that, with the thermal tail attached, the response of the gold foil includes the $5-e v$ resonance. Thus the result is consistent with the actual response.

The lesson to be learned is that, if the wrong trial spectrum is used, and if careful attention is not given to what the code indicates is a better trial, one may conclude that a spectrum that is consistent with the full foil set is not obtainable, or one may end up with a reasonable-looking spectrum only because disagreeable data are discarded. We try to discard only data that we have good reason to believe are in error.

Continuing in the same manner, that is, using a smoothed result as a trial in the next run, we have arrived at the spectrum shape shown in Figure IV.5. The SD for 15 foils is $3.0 \%$.

A parameter commonly used to characterize spectrum properties is the spectral index (SI), defined as the ratio of neutron fluence above $10 \mathrm{keV}$ to that above $3 \mathrm{MeV}$. This is a valuable parameter to use when experiments with silicon-based electronics are being carried out. These devices generally exhibit little displacement damage below $10 \mathrm{keV}$, and if the fluence is being measured with sulfur pellets that have a reaction threshold at about $3 \mathrm{MeV}$, then sulfur pellet measurements together with an SI will provide a measure of the total fluence striking the target to which the devices are 


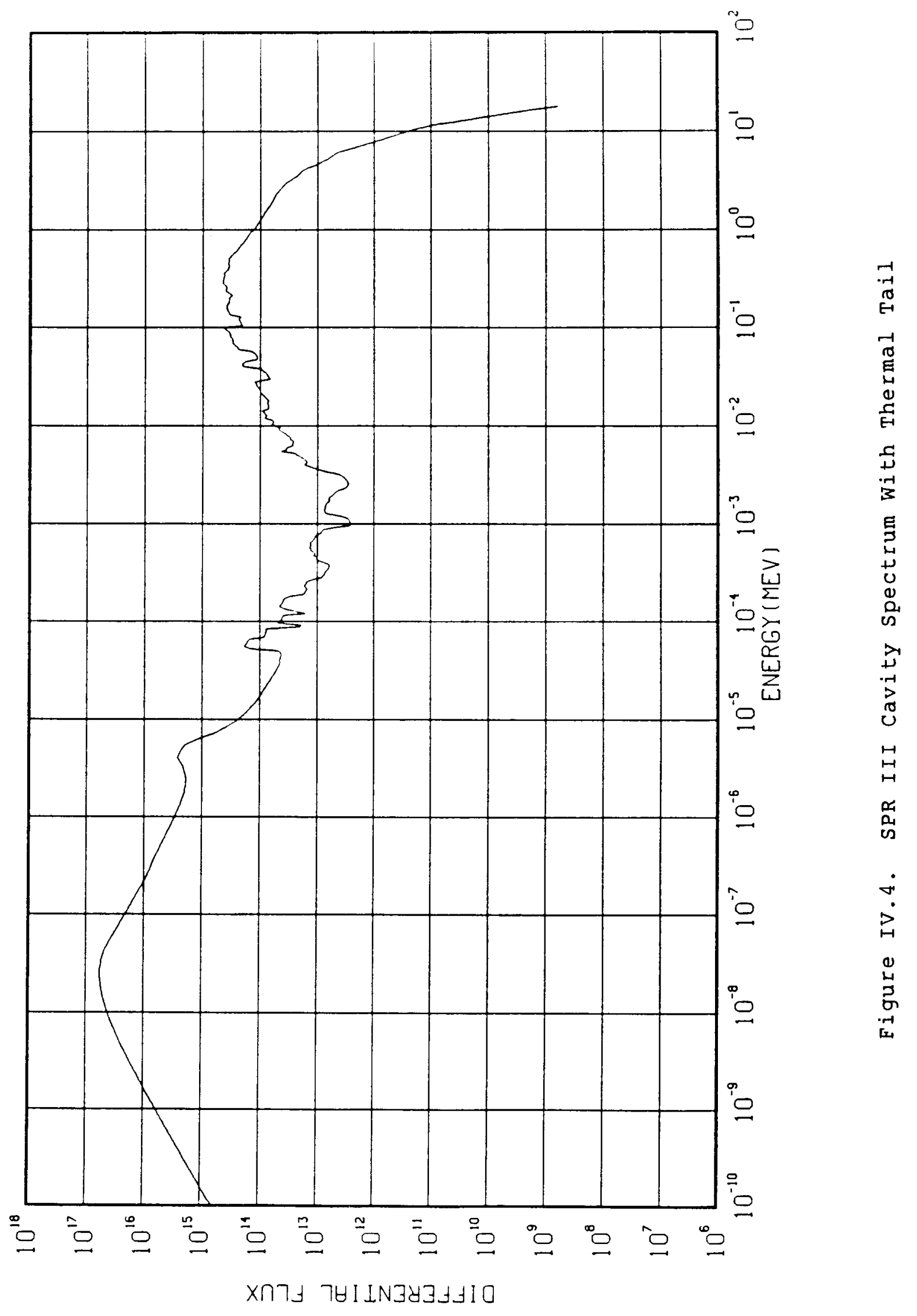




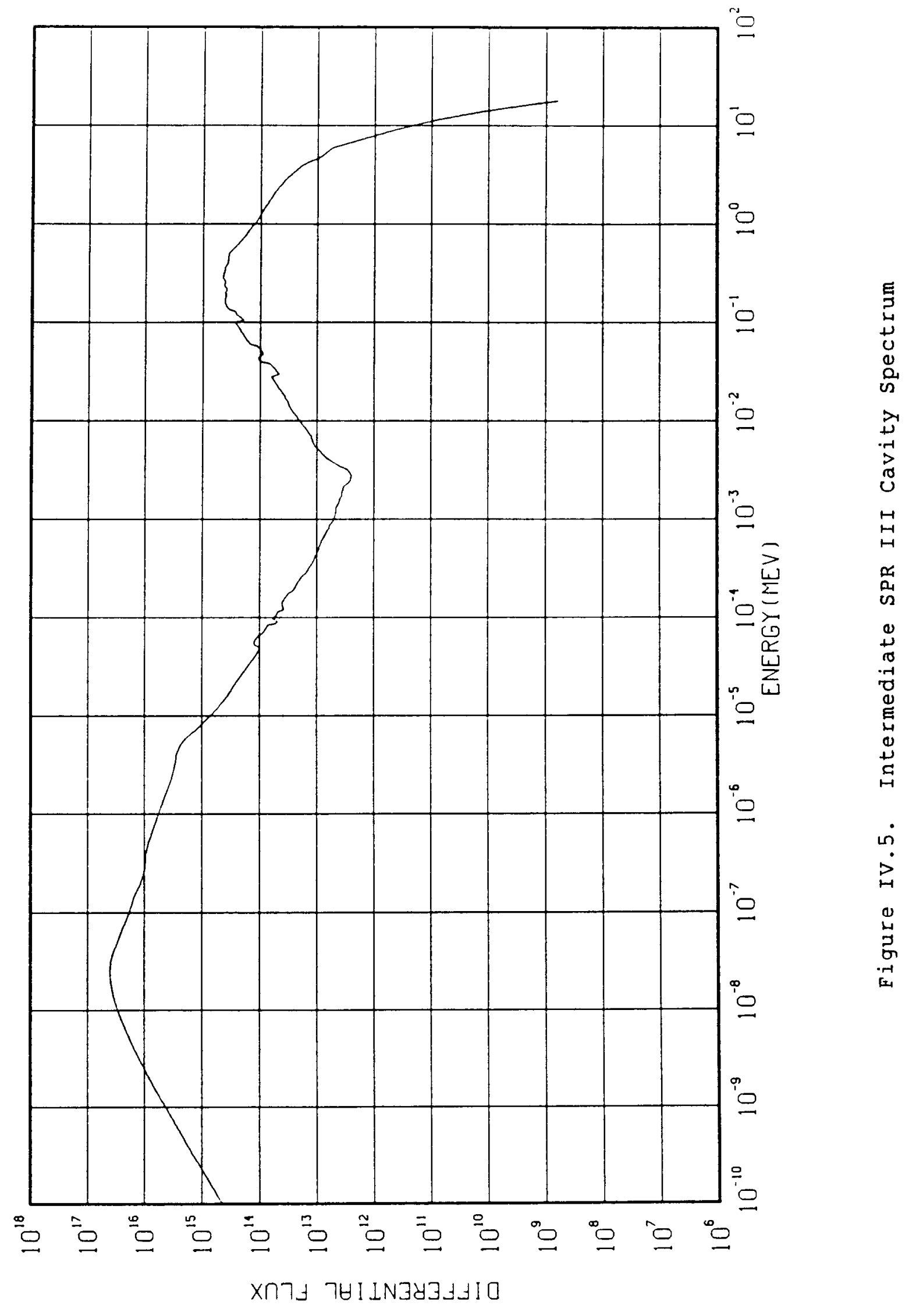


sensitive. The SI for the spectrum in Figure IV.6 is 8.8 . The code also provides other parameter values for this 6.55-MJ pulse. The integral fluences were

$$
\begin{aligned}
& \text { Total }=4.51 \mathrm{E}+14 \mathrm{n} / \mathrm{cm}^{2} \\
& \text { - }>10 \mathrm{keV}=4.506 \mathrm{E}+14 \mathrm{n} / \mathrm{cm}^{2} \\
& \text { - }>3 \mathrm{MeV}=5.134 \mathrm{E}+13 \mathrm{n} / \mathrm{cm}^{2}
\end{aligned}
$$

Despite the thermal tail, very little of the total fluence is below $10 \mathrm{keV}$.

The spectrum values for each configuration measured on the reactors, including the WSMR FBR, are contained in Chapter V. 


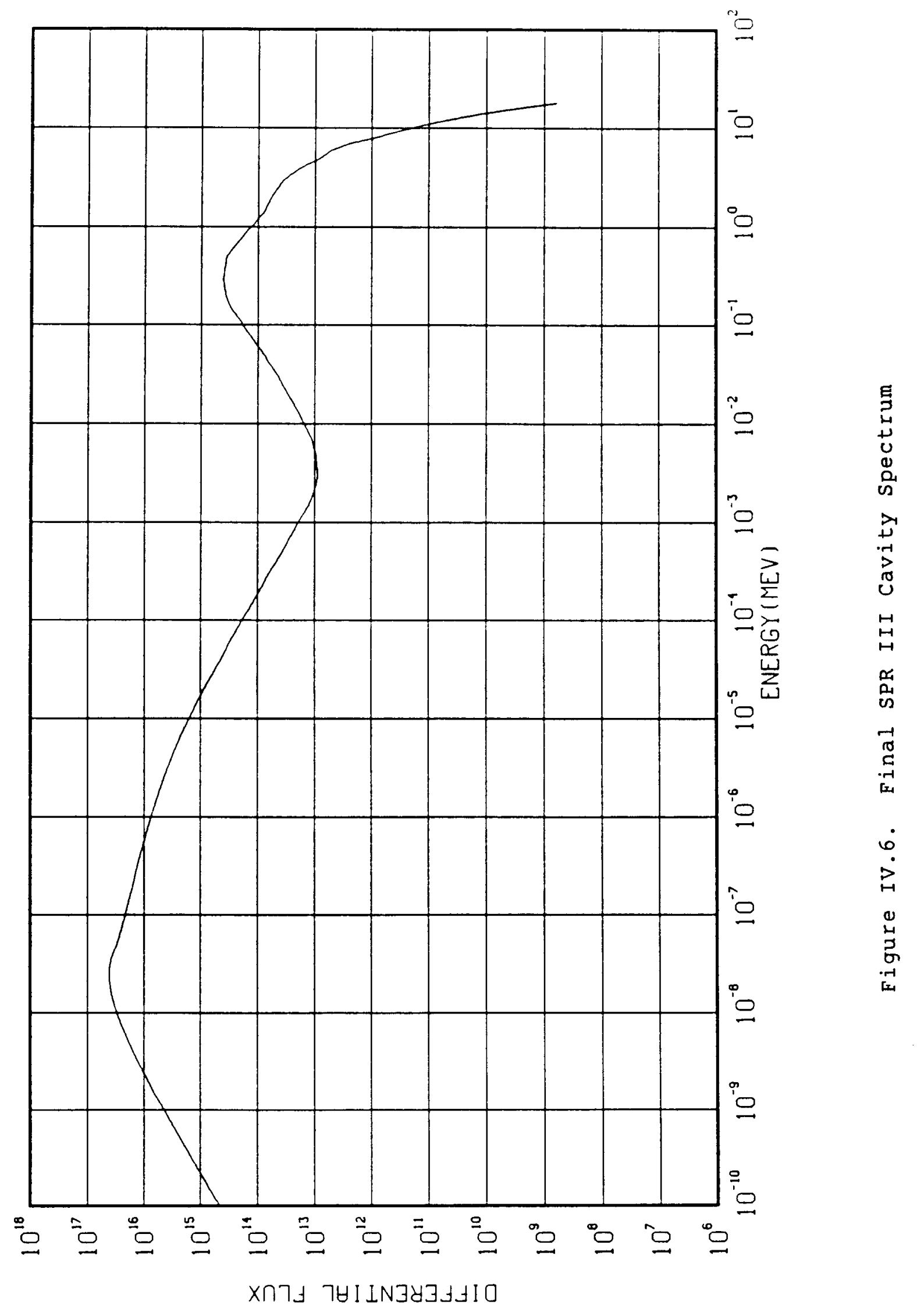




\section{B. Investigation of Solutions by Perturbation of Activities}

We have estimated that the relative accuracy of the activity measurements is approximately 5\%, but in some cases the data appear to be more consistent than that. It is worthwhile to find out how the fluence at each energy, as calculated by SANDII, is altered by $5 \%$ changes in the activity of each foil. As each foil activity was perturbed by $5 \%$, the activities of all the other foils were maintained at their original values. The value of the calculated fluence change was then read from the code output at energies where the particular foil was thought to have strong influence on the spectrum.

Table IV.2 lists each reaction that was studied. Above the line opposite each reaction is a list of energies at which the change in fluence value was read for that run. Below the line are listed the corresponding changes in fluence that resulted from the $5 \%$ change in that foil's activity. All of these values are plotted in Figure IV.7. A boundary line has been drawn through the points that show the greatest deviation. One can see that activity errors of individual foils have little effect between 0.1 and $4 \mathrm{MeV}$. At high energy, where the spectrum decreases steeply and the fluence is small, a large change in fluence is required to change the activity by $5 \%$. The same is true at low energies. At $3 \mathrm{keV}$ in particular, both manganese and sodium dictate that the fluence there changes by an order of magnitude. At $0.3 \mathrm{kev}$, a $5 \%$ change in the manganese activity changes the fluence by a factor of 30 .

The figure shows that, for some regions of the spectrum, small errors in the measured activity may have a very large influence on the fluence because the foil under consideration is responsive over a wide energy range. Thus, it may take a large change in fluence at the particular energy to alter the activity. The compensating feature is that these are the same energy regions in which the exact nature of the spectrum shape is usually least important. This result also means that finding a spectrum that is smoothly consistent to a high degree with foils such as manganese and sodium may be difficult, i.e., small relative activity changes, caused by error in the measurements, may lead to wide variations of spectra in those energy regions where resonances occur. 
Table IV.2

Changes in Fluence for $5 \%$ Changes in Activities

\begin{tabular}{|c|c|c|c|c|c|}
\hline PU239F & $2.0 \mathrm{E}-1$ & $7.2 \mathrm{E}-1$ & 3.0 & & $E(\mathrm{MeV})$ \\
\hline & & 0.6 & 0.7 & & \% Change \\
\hline NA2 3G & $\frac{1.0 E-6}{1.12}$ & $\frac{3.0 \mathrm{E}-3}{10.49}$ & $\frac{3.0 E-2}{4.46}$ & $\frac{2.0 \mathrm{E}-1}{1.84}$ & \\
\hline MG2 4D & $\frac{6.5}{1.32}$ & $\frac{8.0}{2.61}$ & & & \\
\hline AL 27A & $\frac{7.0}{0.93}$ & $\frac{9.0}{2.87}$ & & & \\
\hline AL 27P & $\frac{3.5}{0.78}$ & $\frac{7.0}{1.17}$ & & & \\
\hline $\mathrm{S} 32 \mathrm{P}$ & $\frac{2.5}{0.79}$ & $\frac{3.0}{1.14}$ & $\frac{4.0}{1.75}$ & & \\
\hline MN55G & $\frac{3.0 E-4}{28.7}$ & $\frac{1.0 E-3}{25.8}$ & $\frac{2.0 E-1}{0.7}$ & $\frac{3.0 E-3}{10.5}$ & $\frac{3.0 E-2}{0.78}$ \\
\hline FE54P & $\frac{2.5}{0.7}$ & $\frac{4.0}{1.64}$ & & & \\
\hline FE56P & $\frac{6.0}{3.94}$ & $\frac{8.0}{0.55}$ & & & \\
\hline NI58P & $\frac{2.3}{0.4}$ & $\frac{3.0}{1.04}$ & $\frac{4.0}{1.39}$ & & \\
\hline ZR902 & $\frac{13.0}{4.67}$ & $\frac{14.0}{5.16}$ & & & \\
\hline IN $115 \mathrm{~N}$ & $\frac{1.0}{0.5}$ & $\frac{2.0}{1.35}$ & $\frac{3.0}{0.56}$ & & \\
\hline AU197G & $\frac{5.0 \mathrm{E}-6}{6.21}$ & $\frac{6.0 \mathrm{E}-5}{5.25}$ & $\frac{2.0 \mathrm{E}-1}{0.47}$ & $\frac{7.2 \mathrm{E}-1}{0.39}$ & $\frac{1.5}{0.84}$ \\
\hline $\mathrm{U} 235 \mathrm{~F}$ & $\frac{2.0 \mathrm{E}-1}{0.5}$ & $\frac{7.2 \mathrm{E}-1}{0.44}$ & $\frac{3.0}{0.7}$ & & \\
\hline U2 $38 \mathrm{~F}$ & $\frac{1.5}{1.46}$ & $\frac{2.5}{1.59}$ & & & \\
\hline NP2 $37 \mathrm{~F}$ & $\frac{6.0 \mathrm{E}-1}{0.59}$ & $\frac{1.0 \mathrm{E}-3}{1.75}$ & $\frac{3.0}{0.4}$ & $\frac{2.0 \mathrm{E}-1}{1.0}$ & \\
\hline
\end{tabular}




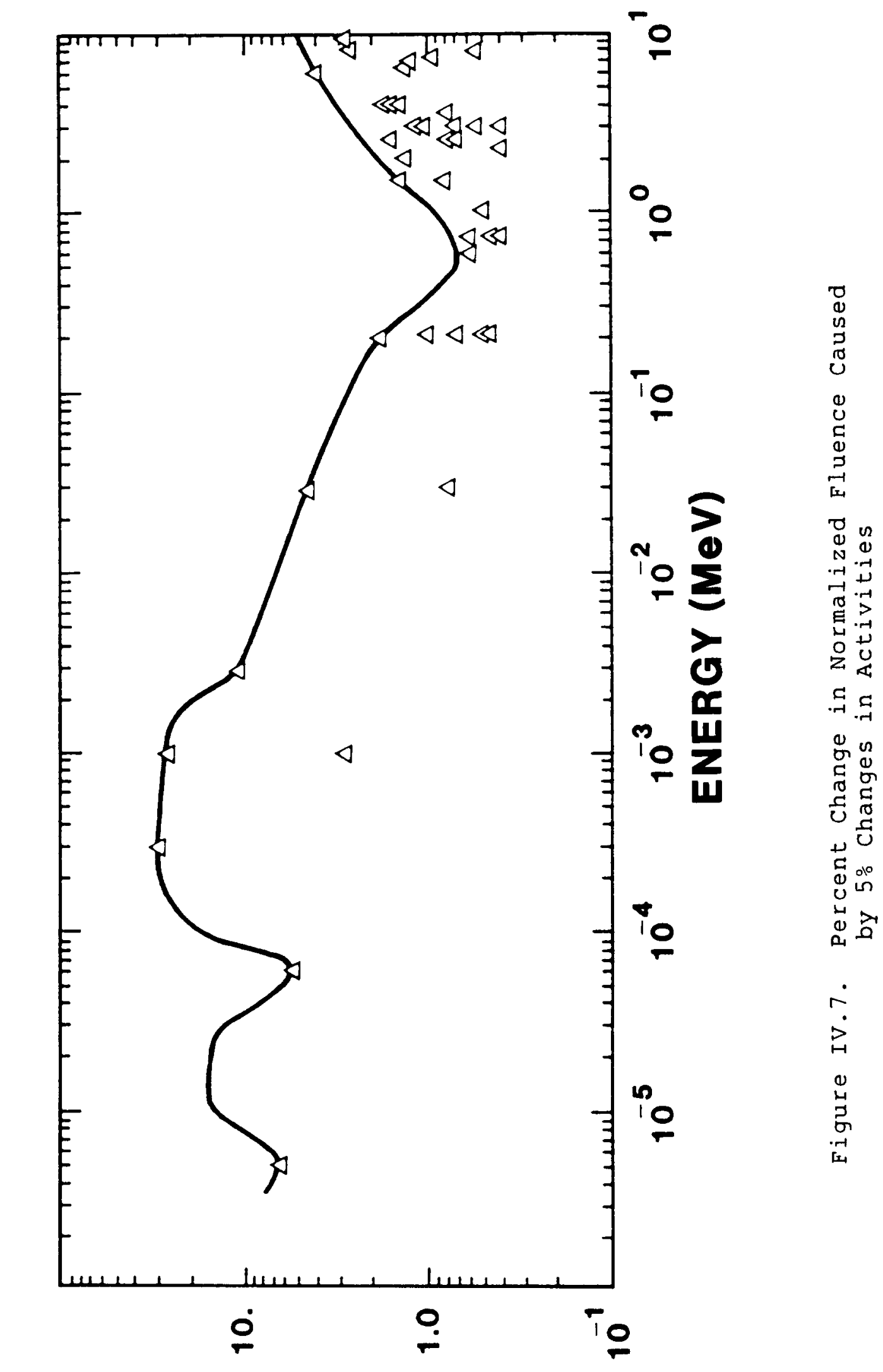

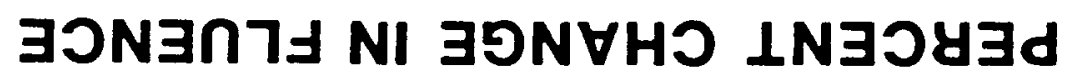




\section{Investigation of solutions by Perturbation of Trial Function}

The question naturally arises, "How accurate is the solution at each energy?" Therefore, it was decided to seek a figure of merit at each energy based on how well SANDII could force a locally perturbed solution back toward the original solutions after a fixed number of iterations. For example, if the trial spectrum is altered by imposing a bulge in an appropriate energy region, and SANDII forces the solution back to the original, unperturbed spectrum in just a few iterations, then one can say that for this data set the solution is well determined or "rigid" at this energy. On the other hand, if the code can only slightly affect the perturbed values after numerous iterations, the fluence is only weakly determined or "soft." As will become evident from the discussion in the rest of this section, a soft fit does not necessarily mean that the spectrum shape is arbitrary in that region; it may just indicate that the approach to the solution is very slow and requires careful observation and small adjustments in the trial to reach it. Our examination of the spectral shapes plotted on log-log paper indicated that, with the foil sets now being used, limited portions of the curve might be approximated by a simple sinusoidal function. The perturbation form that we chose to apply to the spectrum has the form

$$
P(E)=1+B \frac{E_{b}}{E \phi(E)} \sin 2 \pi \frac{\log \frac{E}{E_{b}}}{\log _{\frac{E}{E_{b}}}}
$$

where

$B=$ an arbitrary normalization coefficient

$\mathrm{E}_{\mathrm{b}}=$ energy at which perturbation begins

$E_{\mathrm{f}}=$ energy at which perturbation ends after it has gone through one complete $360^{\circ}$ cycle.

The parameters are illustrated in Figure IV.8.

For a flat initial spectrum, this is a sinusoidal function with a decreasing amplitude in log-log space. P(E) also has the advantage that no matter what the shape of the spectrum $\phi(E)$, the integral from $E_{b}$ to $E_{f}$ is just equal to 1 , so that the perturbation does not change the normalization of the whole trial and thereby shift the trial spectrum values outside the perturbation interval. The derivation of this function is given in Appendix A. In the analysis, we are interested in the fluence value at the point where the 


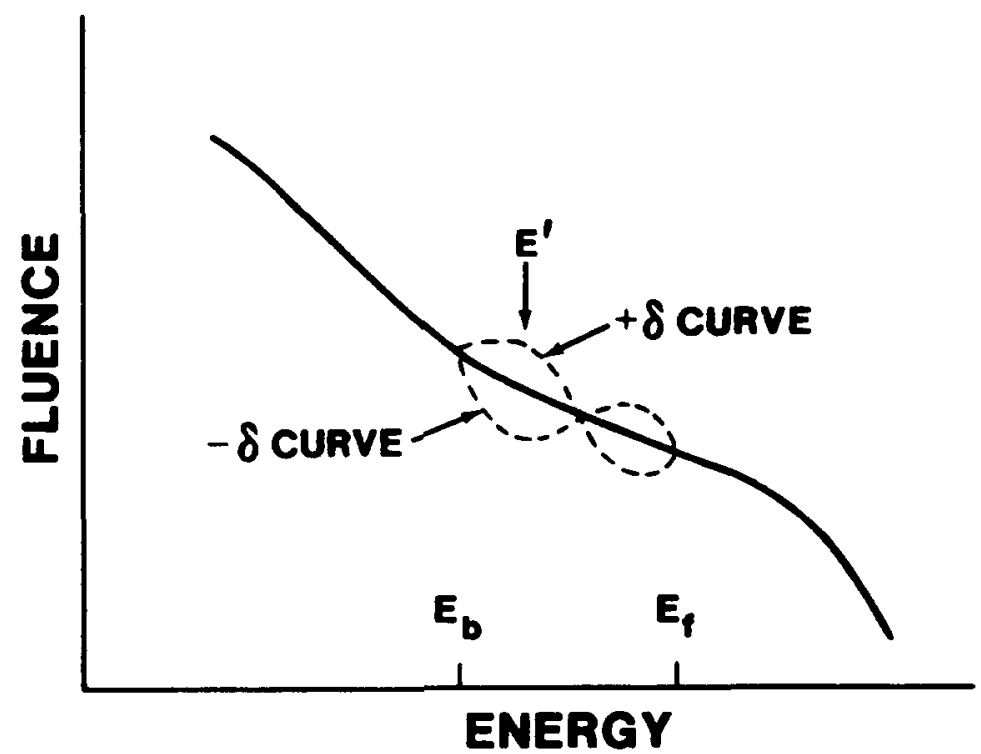

Figure IV.8. Perturbation Form

perturbation is a maximum (defined as $E^{\prime}$ ), that is, where the sine function equals 1 . This definition leads to

$$
E^{\prime}=E_{b}\left(\frac{E_{f}}{E_{b}}\right)^{1 / 4}
$$

where $E^{\prime}=$ energy at which perturbation is a maximum.

If $\delta$ is defined as the fractional change in fluence level at $E^{\prime}$, the value of $B$ can be found in relation to the chosen magnitude of the perturbation:

$$
B=\delta \frac{E^{\prime}}{E_{b}} \phi\left(E^{\prime}\right)
$$

where $\phi\left(E^{\prime}\right)=$ value of the initial spectrum at $E^{\prime}$. The perturbation function then becomes 


$$
P(E)=1+\delta \frac{E^{\prime} \phi\left(E^{\prime}\right)}{E \phi(E)} \sin 2 \pi \frac{\log \frac{E}{E_{b}}}{\log \frac{E_{f}}{E_{b}}}
$$

for any value of $E$ in the interval $E_{b} \rightarrow E_{f}$.

The program PERTURB calculates all the perturbed fluence values in the trial once a value of $\delta$ and a set of $E^{\prime}$ values are chosen. For each chosen value of $E^{\prime}$, the fluence values of the perturbed trial are calculated at the energies tabulated for the trial function. The analyst, therefore, just substitutes the perturbed trial fluence values into the interval $E_{b} \leq E \leq E_{f}$ and runs SANDII for that perturbation around $E^{\prime}$. The trial is first perturbed upward a maximum fraction $\delta$ and then downward by the same fraction. The proximity of the two fluence values after, say, 15 SANDII iterations is then evaluated. The fractional difference between two solutions is $2\left(\phi_{+}-\phi_{-}\right) /\left(\phi_{+}+\phi_{-}\right)$, where $\phi_{+}$and $\phi_{-}$are the positively and negatively perturbed solutions at $E^{\prime}$, respectively. The initial fractional difference is $n_{0}$; the final fractional difference is $\mathrm{n}_{\mathrm{f}}$. For example, with $\delta=0.2$, the $\mathrm{n}_{0}=0.4$ at $\mathrm{E}^{\prime}$. If, after 15 iterations, $n_{f}=0.25$, then the ratio of the differences $\mathrm{R}_{\mathrm{d}}=\mathrm{n}_{\mathrm{f}} / \mathrm{n}_{0}=0.625$. Table IV.3 contains a list of $R_{d}$ for values of $E^{\prime}$ obtained with $\delta= \pm 0.2$ and a SPR III cavity trial function.

The ratio $R_{d}$ is plotted in Figure IV.9. It is evident that the code is able to restore the perturbed trial at some energies far better than at others. The curve reflects the ability of SANDII to find a solution at each energy for this foil set. At low energies, the solution at particular resonances is very rigid. At high energies, the structure imposed by individual cross sections is not evident, but the many overlapping threshold reactions permit the code to determine solutions very well. Between resonances in the middle and lower energies, however, the code does not influence the trial very well at all.

The fact that the code can narrow the gap between solutions at least a little at each energy led to an attempt to predict where the two solutions would ultimately converge if the code were allowed to iterate forever. The reason for pursuing this extrapolation is that, although the solutions at $E^{\prime}$ may be soft and the approach to a solution may be very slow, these facts do not mean that this solution is not ultimately well defined. In other words, given enough iterations the two solutions may converge on a unique fluence value. Figure IV.10.a illustrates how the solutions might converge in a high-response, rapid convergence case, while Figure IV.10.b illustrates a low-response, slow-convergence case. In Figure IV.10.a the convergence is so rapid that after only a 
Table IV. 3

Fractional Differences After 15 Iterations

for SPR III Cavity spectrum

\begin{tabular}{|c|c|c|c|c|c|c|c|c|}
\hline $\begin{array}{l}\mathrm{E}^{\prime} \\
\text { Index }\end{array}$ & $\begin{array}{c}E^{\prime} \\
(\mathrm{MeV})\end{array}$ & $\phi_{0}$ & $\phi_{+0}(a)$ & $\phi_{-o}(b)$ & $\phi_{+}$ & $\phi_{-}$ & $\mathrm{n}_{\mathrm{f}}$ & $\mathrm{R}_{\mathrm{d}}$ \\
\hline 1 & $1.0 \mathrm{E}-7$ & $6.0 \mathrm{E}+1$ & 7. $2 E+1$ & $4 \cdot 8 \mathrm{E}+1$ & $6.339 E+1$ & $4.334 E+1$ & 0.376 & 0.940 \\
\hline 2 & $3.0 \mathrm{E}-7$ & $3.7 \mathrm{E}+1$ & $4.44 E+1$ & $2.96 \mathrm{E}+1$ & $3.954 E+1$ & $2.720 \mathrm{E}+1$ & 0.370 & 0.925 \\
\hline 3 & $1.0 \mathrm{E}-6$ & $2.0 \mathrm{E}+1$ & $2.40 \mathrm{E}+1$ & $1.60 \mathrm{E}+1$ & $2.243 E+1$ & $1.532 \mathrm{E}+1$ & 0.377 & 0.943 \\
\hline 4 & $3.0 \mathrm{E}-6$ & $1.0 \mathrm{E}+1$ & $1.20 \mathrm{E}+1$ & 8.0 & $1.110 \mathrm{E}+1$ & 7.709 & 0.361 & 0.902 \\
\hline 5 & $5.0 \mathrm{E}-6$ & 6.9 & 8.28 & 5.52 & 6.756 & 6.340 & 0.64 & 0.160 \\
\hline 6 & $1.0 \mathrm{E}-5$ & 4.1 & 4.92 & 3.28 & 4.579 & 3.121 & 0.379 & 0.948 \\
\hline 7 & $3.0 E-5$ & 1.55 & 1.86 & 1.24 & 1.711 & 1.175 & 0.371 & 0.927 \\
\hline 8 & $1.0 \mathrm{E}-4$ & $5.0 \mathrm{E}-1$ & $6.00 \mathrm{E}-1$ & $4.00 \mathrm{E}-1$ & $5.641 \mathrm{E}-1$ & $3.839 \mathrm{E}-1$ & 0.380 & 0.950 \\
\hline 9 & $3.0 E-4$ & $1.6 \mathrm{E}-1$ & $1.92 \mathrm{E}-1$ & $1.28 \mathrm{E}-1$ & $1.784 \mathrm{E}-1$ & $1.398 \mathrm{E}-1$ & 0.243 & 0.607 \\
\hline 10 & $1.0 E-3$ & $5.0 \mathrm{E}-2$ & $6.00 \mathrm{E}-2$ & $4.00 E-2$ & $5.917 E-2$ & $4.098 \mathrm{E}-2$ & 0.363 & 0.907 \\
\hline 11 & $3.0 E-3$ & $2.4 \mathrm{E}-2$ & $2.88 E-2$ & $1.92 \mathrm{E}-2$ & $2.322 E-2$ & $1.627 \mathrm{E}-2$ & 0.352 & 0.880 \\
\hline 12 & $1.0 \mathrm{E}-2$ & $3.5 E-2$ & $4.20 E-2$ & $2.80 \mathrm{E}-2$ & $4.377 E-2$ & $2.990 \mathrm{E}-2$ & 0.377 & 0.942 \\
\hline 13 & $3.0 E-2$ & $1.08 \mathrm{E}-1$ & $1.30 \mathrm{E}-1$ & $8.64 E-1$ & $1.241 \mathrm{E}-1$ & $8.506 \mathrm{E}-2$ & 0.373 & 0.933 \\
\hline 14 & $1.0 \mathrm{E}-1$ & $4.5 \mathrm{E}-1$ & $5.40 \mathrm{E}-1$ & $3.60 \mathrm{E}-1$ & $6.024 \mathrm{E}-1$ & $4.213 \mathrm{E}-1$ & 0.354 & 0.885 \\
\hline 15 & $3.0 \mathrm{E}-1$ & $9.2 \mathrm{E}-1$ & $1.10 \mathrm{E}-0$ & $7.36 \mathrm{E}-1$ & 1.066 & $8.299 \mathrm{E}-1$ & 0.249 & 0.623 \\
\hline 16 & $5.0 \mathrm{E}-1$ & 7. $0 \mathrm{E}-1$ & $8.40 \mathrm{E}-1$ & $5.60 \mathrm{E}-1$ & $8.249 \mathrm{E}-1$ & $6.306 \mathrm{E}-1$ & 0.267 & 0.668 \\
\hline 17 & 1.0 & $2.8 \mathrm{E}-1$ & $3.36 \mathrm{E}-1$ & $2.24 \mathrm{E}-1$ & $3.056 E-1$ & $2.564 \mathrm{E}-1$ & 0.175 & 0.438 \\
\hline 18 & 2.0 & $1.45 \mathrm{E}-1$ & $1.74 \mathrm{E}-1$ & $1.16 \mathrm{E}-1$ & $1.386 \mathrm{E}-1$ & $1.221 \mathrm{E}-1$ & 0.127 & 0.318 \\
\hline 19 & 3.0 & $9.2 \mathrm{E}-2$ & $1.10 \mathrm{E}-2$ & $7.36 \mathrm{E}-2$ & $9.296 \mathrm{E}-2$ & $8.550 \mathrm{E}-2$ & 0.084 & 0.210 \\
\hline 20 & 5.0 & $1.4 \mathrm{E}-2$ & $1.68 \mathrm{E}-2$ & 1. $12 \mathrm{E}-2$ & $1.415 \mathrm{E}-2$ & $1.253 \mathrm{E}-2$ & 0.121 & 0.302 \\
\hline 21 & 7.0 & $5.5 E-3$ & $6.60 E-3$ & $4.40 E-3$ & $5.194 E-3$ & $6.259 \mathrm{E}-3$ & 0.186 & 0.465 \\
\hline (a) & \multicolumn{8}{|c|}{$\phi_{+o}=(1.2) \phi_{0}$} \\
\hline (b) & \multicolumn{8}{|c|}{$\phi_{-o}=(0.8) \phi_{0}$} \\
\hline
\end{tabular}




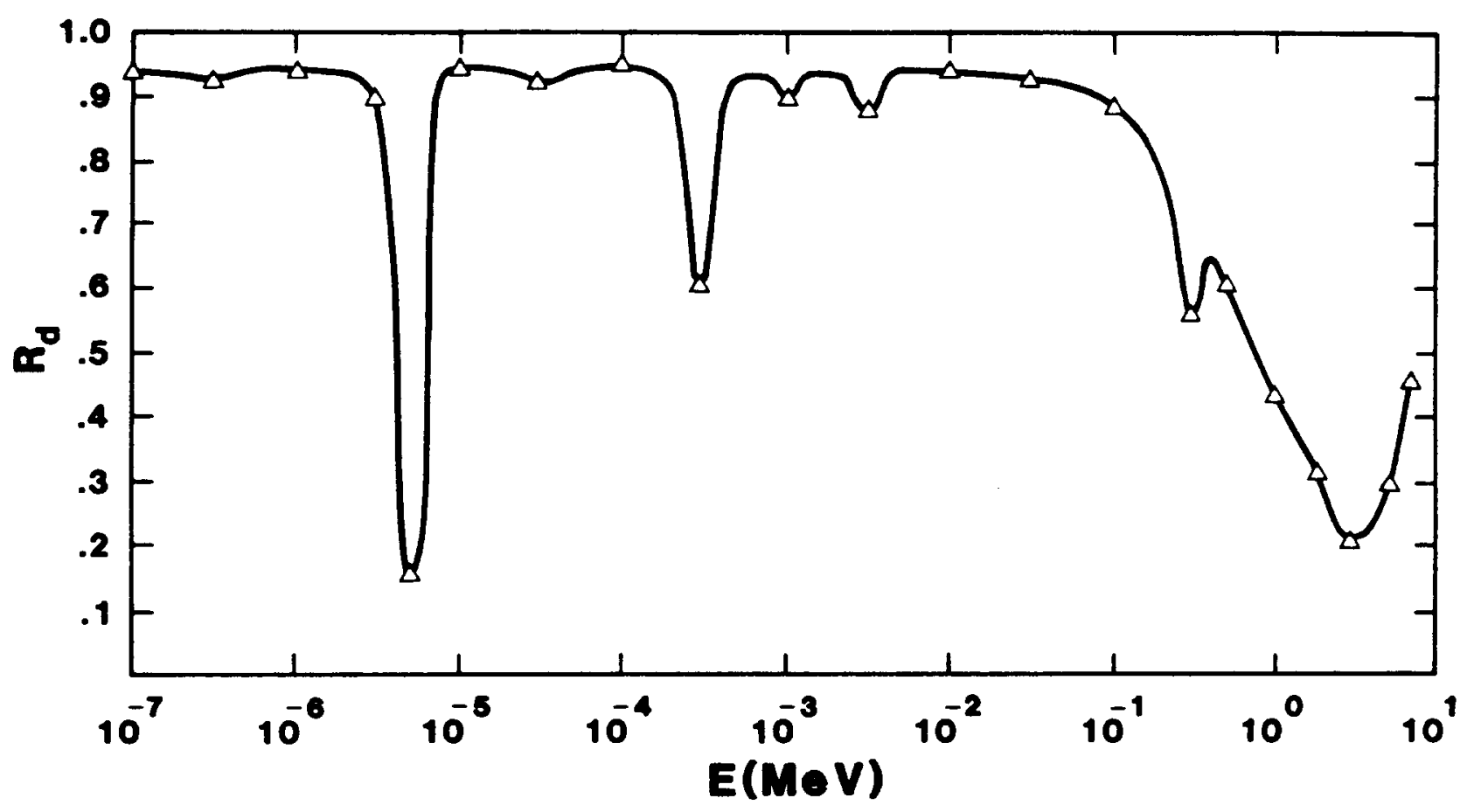

Figure IV.9. Fractional Difference Ratio Versus Energy

few iterations, $n_{f}$, the solutions are very close together. Extrapolation the rest of the way to the convergence point will be very easy and quite accurate. In Figure IV.10.b the convergence is slow. After a practical number of iterations, $\mathrm{n}_{\mathrm{s}}$, have been carried out, the difference is still large. Extrapolating inward to the final convergence point, though still useful, will be much more difficult and subject to the effects of errors. In addition, it is obvious that, in general, errors in cross-section values will be larger in energy regions where the cross sections for the relevant reactions are low and difficult to measure. Thus, the difficulty of extrapolating inward to the "real" fluence level is compounded.

The qualitative form of the convergence of the perturbed spectrum solution is illustrated in Figures IV.11 and IV.12. In Appendix $B$ the formulas for the convergence point in the $x, \phi$ space are derived. They are

$$
\phi=\phi_{0} \frac{(1+\delta) \phi_{-}-(1-\delta) \phi_{+}}{\phi_{-}+2 \delta \phi_{0}-\phi_{+}}
$$




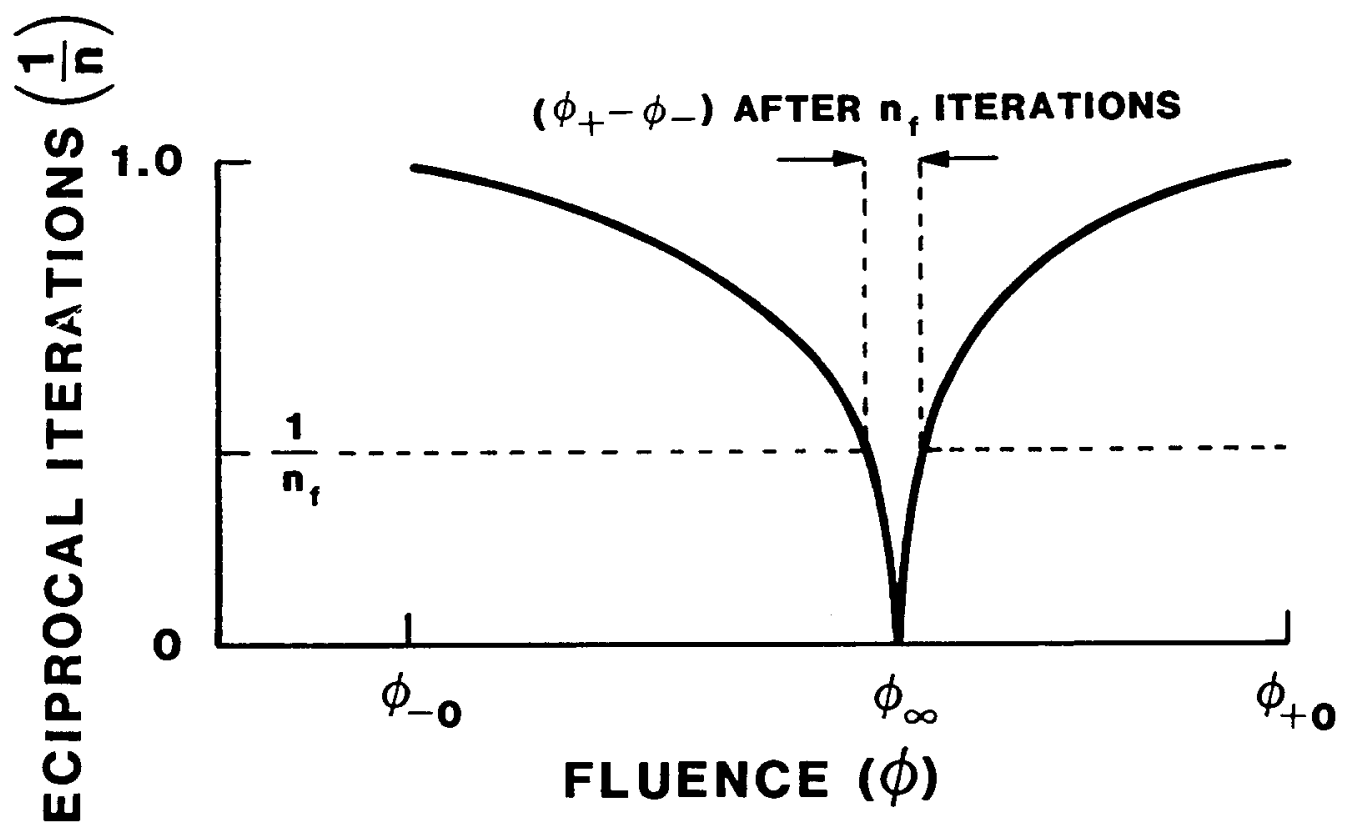

(a) FAST CONVERGENCE AT E', WHERE RESPONSE IS HIGH.

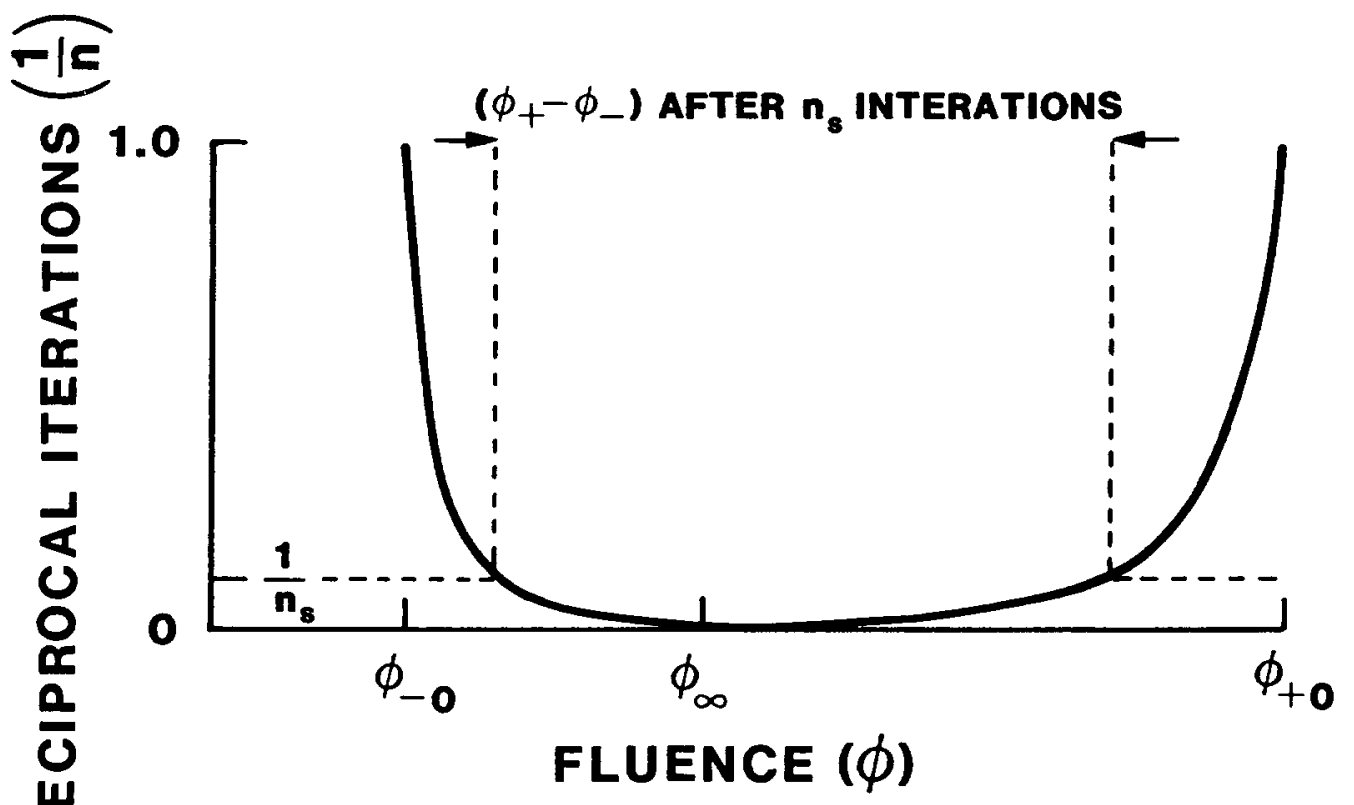

(b) SLOW CONVERGENCE AT E $E_{2}^{\prime}$ WHERE RESPONSE IS LOW.

Figure IV.10. The Approach to a solution 


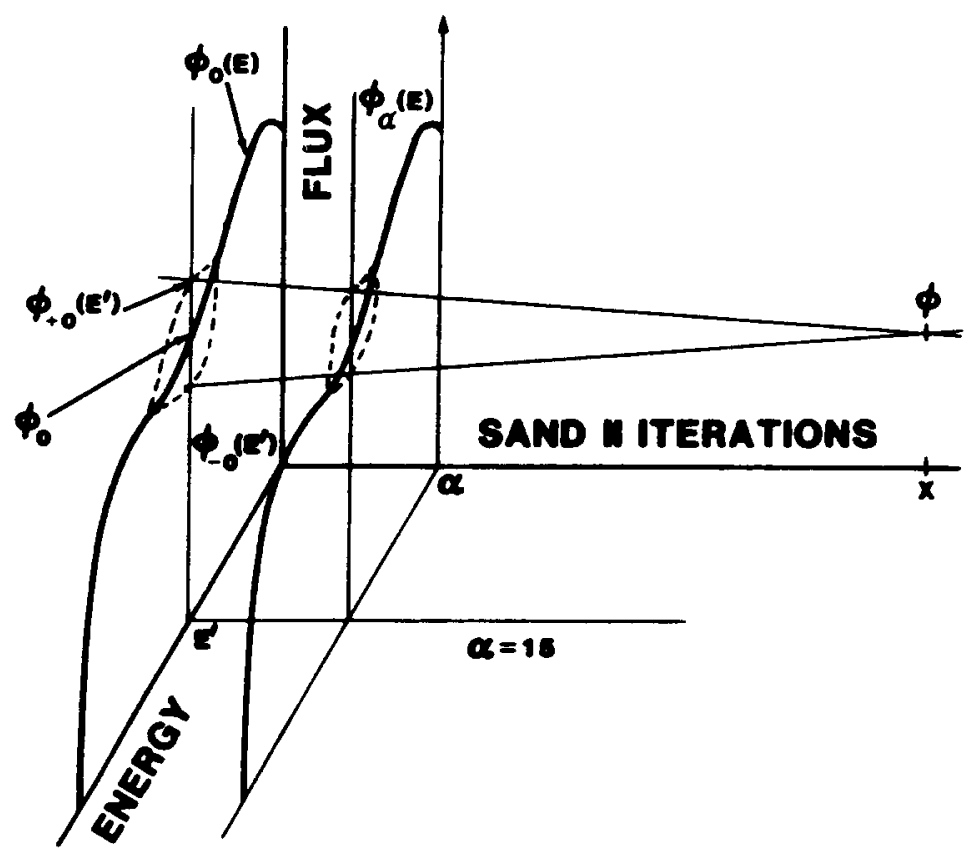

Figure IV.11. Convergence of Solutions for Perturbed Trials

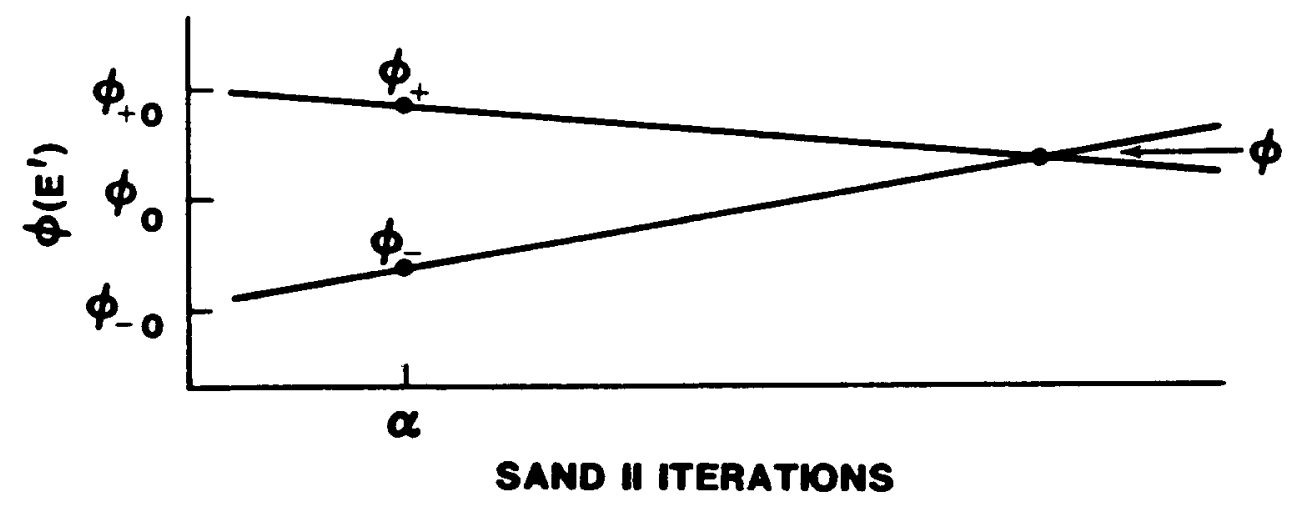

Figure IV.12. Convergence of Solutions for Perturbed Trials at Chosen Energy E' 
and

$$
\frac{x}{\alpha}=\frac{2 \delta \phi_{0}}{\phi_{-}+2 \delta \phi_{0}-\phi_{+}}
$$

where

$$
\begin{aligned}
\mathrm{x} & =\text { number of iterations at convergence point } \\
\phi & =\text { fluence at convergence point, } \mathrm{n} / \mathrm{cm}^{2} \\
\alpha & =\text { final SANDI iteration number (taken to be 15) } \\
\phi_{0} & =\text { initial fluence at } \mathrm{E}^{\prime}, \mathrm{n} / \mathrm{cm}^{2} \\
\phi_{+} & =\phi_{O}(1+\delta), \mathrm{n} / \mathrm{cm}^{2} \\
\phi_{-} & =\phi_{O}(1-\delta), \mathrm{n} / \mathrm{cm}^{2} \\
\phi_{+} & =\text {SANDII solution for trial with } \phi_{+0}, \mathrm{n} / \mathrm{cm}^{2} \\
\phi_{-} & =\text {SANDII solution for trial with } \phi_{-0}, \mathrm{n} / \mathrm{cm}^{2} .
\end{aligned}
$$

The ratio $x / \alpha$ provides a measure of how many iterations would be required to reach a solution at this energy. The extrapolated $\phi$ values and the $x / \alpha$ values with respect to $E^{\prime}$ are included in Table IV.4. The comparisons of $\phi$ with $\phi_{0}$ provide insight into either how good the trial spectrum is (this trial spectrum is actually the best solution spectrum that had been found by the procedure discussed in section $A$ of this chapter) or how well the extrapolation procedure is working. Clearly, if one has to extrapolate a very long distance (large number of iterations), the error in the calculated $\phi$ then after $x$ iterations the error is roughly $\frac{x}{\alpha} \Delta$. )

Figure IV.9 shows that below 0.5 MeV (where the Np237(n,f)FP sensitivity becomes insignificant), the approach to a well-defined solution for this foil set and spectrum is very slow. Furthermore, because most usable reactions at low energies are caused by resonance absorption, any trial value between the resonance responses will not be altered by SANDII while it is approaching the designated SD. It is unreasonable to establish an "uncertainty band" in these regions based on how rapidly the positively and negatively perturbed trials approach each other. It is far better to smoothly connect the uncertainty band boundaries that are well determined and to try to reduce the uncertainties at well-determined points on the spectrum. 
Table IV. 4

Extrapolated Fluence Convergence Points

$$
\begin{gathered}
\phi=\phi_{0} \frac{\phi_{-}(1.2)-\phi_{+}(0.8)}{\phi_{-}+0.4 \phi_{0}-\phi_{+}} \equiv \phi \cdot C \\
\frac{x}{\alpha}=\frac{0.4 \phi_{0}}{\phi_{-}+0.4 \phi_{0}-\phi_{+}}
\end{gathered}
$$

\begin{tabular}{|c|c|c|c|c|c|}
\hline $\begin{array}{c}E^{\prime} \\
\text { Index }\end{array}$ & $\phi_{0}$ & C & $\phi$ & $\frac{x}{\alpha}$ & \\
\hline 1 & $6.0 \mathrm{E}+1$ & 0.328 & 1. $96 \mathrm{E}+1$ & 6.08 & \\
\hline 2 & $3.7 E+1$ & 0.410 & 1. $52 \mathrm{E}+1$ & 6.02 & \\
\hline 3 & $2.0 \mathrm{E}+1$ & 0.494 & 9.89 & 8.99 & \\
\hline 4 & 1. $0 E+1$ & 0.609 & 6.09 & 6.57 & \\
\hline 5 & 6.9 & 0.939 & 6.48 & 1.18 & \\
\hline 6 & 4.1 & 0.451 & 1.84 & 9.01 & \\
\hline 7 & 1.55 & 0.491 & $7.61 \mathrm{E}-1$ & 7.38 & \\
\hline 8 & 5. $0 \mathrm{E}-1$ & 0.475 & $2.37 \mathrm{E}-1$ & 10.10 & \\
\hline 9 & $1.6 \mathrm{E}-1$ & 0.986 & $1.57 \mathrm{E}-1$ & 2.52 & \\
\hline 10 & $5.0 \mathrm{E}-2$ & 1.016 & $5.08 \mathrm{E}-2$ & 11.05 & (a) \\
\hline 11 & $2.4 \mathrm{E}-2$ & 0.358 & $8.59 \mathrm{E}-3$ & 3.62 & (b) \\
\hline 12 & $3.5 E-2$ & 6.656 & $2.33 E-2$ & 107.7 & (c) \\
\hline 13 & $1.08 E-1$ & 0.671 & $7.25 \mathrm{E}-2$ & 10.38 & \\
\hline 14 & 4. $5 E-1$ & $\infty$ & & & (d) \\
\hline 15 & $9.2 \mathrm{E}-1$ & 1.085 & $9.97 \mathrm{E}-1$ & 2.79 & \\
\hline 16 & $7.0 \mathrm{E}-1$ & 1.130 & $7.91 \mathrm{E}-1$ & 3.27 & \\
\hline 17 & $2.8 \mathrm{E}-1$ & 1.006 & $2.81 \mathrm{E}-1$ & 1.78 & \\
\hline 18 & 1. $45 \mathrm{E}-1$ & 0.859 & 1. $25 \mathrm{E}-1$ & 1.40 & \\
\hline 19 & $9.2 \mathrm{E}-2$ & 0.962 & $8.85 E-2$ & 1.25 & \\
\hline 20 & 1. $4 \mathrm{E}-2$ & 0.933 & $1.31 \mathrm{E}-2$ & 1.41 & \\
\hline 21 & $5.5 E-3$ & 1.028 & $5.65 \mathrm{E}-3$ & 0.67 & \\
\hline
\end{tabular}

(a) Weakly determined, but a good guess.

(b) Strongly determined, but a bad guess.

(c) Both poorly determined and a poor guess.

(d) These never met for positive extrapolations. 
One of the primary reasons that the resulting spectrum appears to be so insensitive to local perturbations in the trial is that some reactions are sensitive in two or more regions. SANDII will attempt to alter the trial everywhere that a disagreeing reaction is sensitive. It can do so particularly well where the sensitivity of other foils is low (i.e., if not constrained by other foils). This effect is exhibited by the code's attempt to fit the trial to the sodium activity shown in Figure IV.4. Sodium is sensitive to this spectrum at thermal and epithermal energies, at about $3 \mathrm{keV}$, between 10 and $100 \mathrm{keV}$, and up to about $2 \mathrm{MeV}$. If a trial is too high in a region of low sensitivity, the code will change the spectrum most rapidly where sodium is more sensitive. In this manner the trial spectrum is altered a minimum amount. However, there is no general justification for only changing the spectrum where the response is high and where the spectrum will be changed the least. This is an example of a case in which the spectrum may have to be changed a very large amount in a region where sensitivity is low, in order to match the activities required and to approach physical reality. SANDII cannot directly correct for this deficiency, but it does provide the necessary clues. If the spectrum is forced to smoothly match the gold, manganese, and neptunium activities where these foils are sensitive, then the only place where the spectrum can be altered to fit sodium is in the region between 1 and $300 \mathrm{keV}$. It is the application of this kind of information which led from the fit shown in Figure IV.5 to that shown in Figure IV. 6.

Obviously, the more reactions that can be used with accuracy, the better determined the spectrum will be. Furthermore, even a small error in the measured activity or cross section may cause either a large change in spectrum values or an inability to find any smooth solution. Good cross sections and accurate activity data are the most important factors in the acquisition of reasonable spectra.

\section{Interactive Method for Spectrum Adjustment}

The attempt to devise a purely mechanical means for compensating the deficiencies of SANDII described in the last section has only been partially successful and has not led to an improved algorithm into which one can simply insert activities and arbitrary trials to obtain appropriate solutions. We have been far more successful by working interactively with the code so that the experimenter's knowledge can be injected into the adjustment process as it is needed. This also allows one to recognize important experimental errors and mistakes in the analysis.

This process is further illustrated by the procedures used to obtain the free-field leakage spectrum from SPR III at 
17 inches from the axis of the core. In this case the starting trial is flat ( $\phi=1.0$ everywhere). Figure IV.13 shows the result of SANDII's adjustment process. Despite the poor results at intermediate and low energies, the SI is a fairly reasonable 7.5 , because the $S I$ is related only to the fluence above 1E-2 MeV. Above this energy the spectrum is fairly smooth. The initial SD is $221 \%$, and the final SD is $3.43 \%$.

The next step is to smooth this result to make a new trial. The simplest way is to draw a smooth curve connecting points where the foil-set response is high. The smoother curve in the figure marks the second trial, which approaches the first result at the principal resonances of gold, manganese, and sodium. The shape above $1 \mathrm{E}-1 \mathrm{MeV}$ is just a smoothed curve following the general trend. This trial yields the spectrum depicted in Figure IV.14. Already the improvement is substantial. The initial SD is $21 \%$, the final SD equals $3.32 \%$ after only 10 iterations, and the SI is 7.7 .

Now, more subtle clues can be used for the next trial. The peak at the gold resonance and the dips at sodium resonances show that the previous guess was too low at low energies and too high at intermediate energies. Also, the peaks at the manganese resonances indicate that the trial should be higher there. The region between $3 E-3$ and $5 E-1$ MeV has particularly weak response for this foil set and spectrum; we have found, however, because of the manganese, sodium, and neptunium responses at the edges of this region and the flat responses of plutonium and uranium across it, a certain shape can be found which is compatible with all these reactions. The manganese and sodium show that the spectrum continues to trend downward with energy to $3 \mathrm{E}-3 \mathrm{MeV}$. The flat plutonium and uranium responses $f i x$ the integral of the activities in the region, so that any shape preserving that integral is acceptable to these two reactions. If one limits solutions to smooth curves with a downward trend that ultimately fits the neptunium response at $0.5 \mathrm{MeV}$, there must be a dip at about 1E-2 MeV and a peak at about $0.1 \mathrm{MeV}$. When slight depressions were placed in the trial at $1 \mathrm{E}-2 \mathrm{MeV}$, followed by humps at about $0.1 \mathrm{MeV}$, the initial fit improved steadily. The qualitative progress is illustrated in Figure IV.15.

After a few additional trials with SANDII, the spectrum shown in Figure IV.16 was obtained. This is the result of a trial with initial SD of $3.76 \%$ and $f$ inal $S D$ of $0.81 \%$ after 15 iterations. Actually, the spectrum shape settled within 3 iterations to an SD of $1.0 \%$, after which progress was slow. We believe that the late slow progress indicates that the relative accuracy of the measured activities in this experiment is a few percent. The curve is remarkably smooth for this quality of fit and is very stable. Table IV.5 compares the measured and calculated activities. 


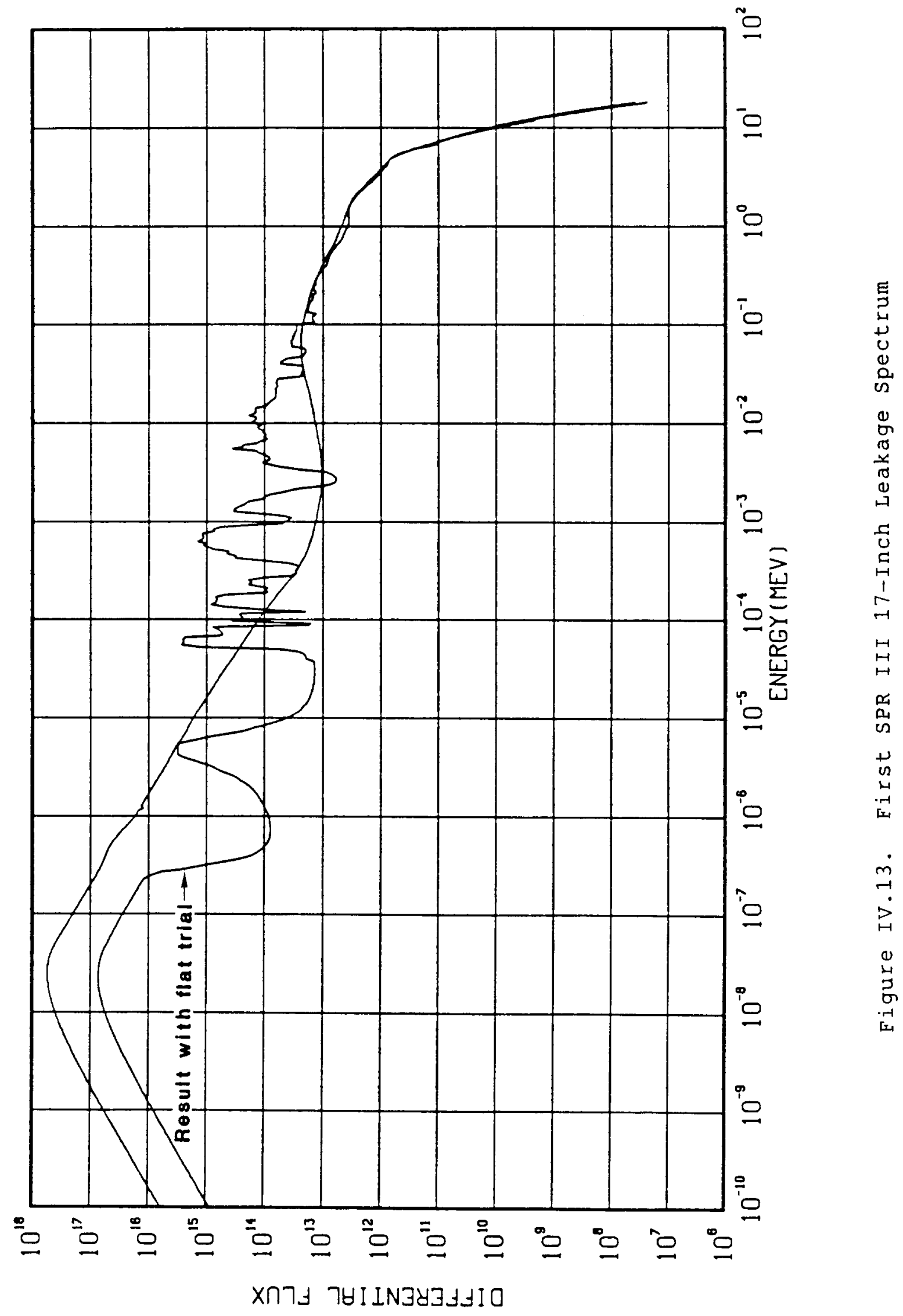




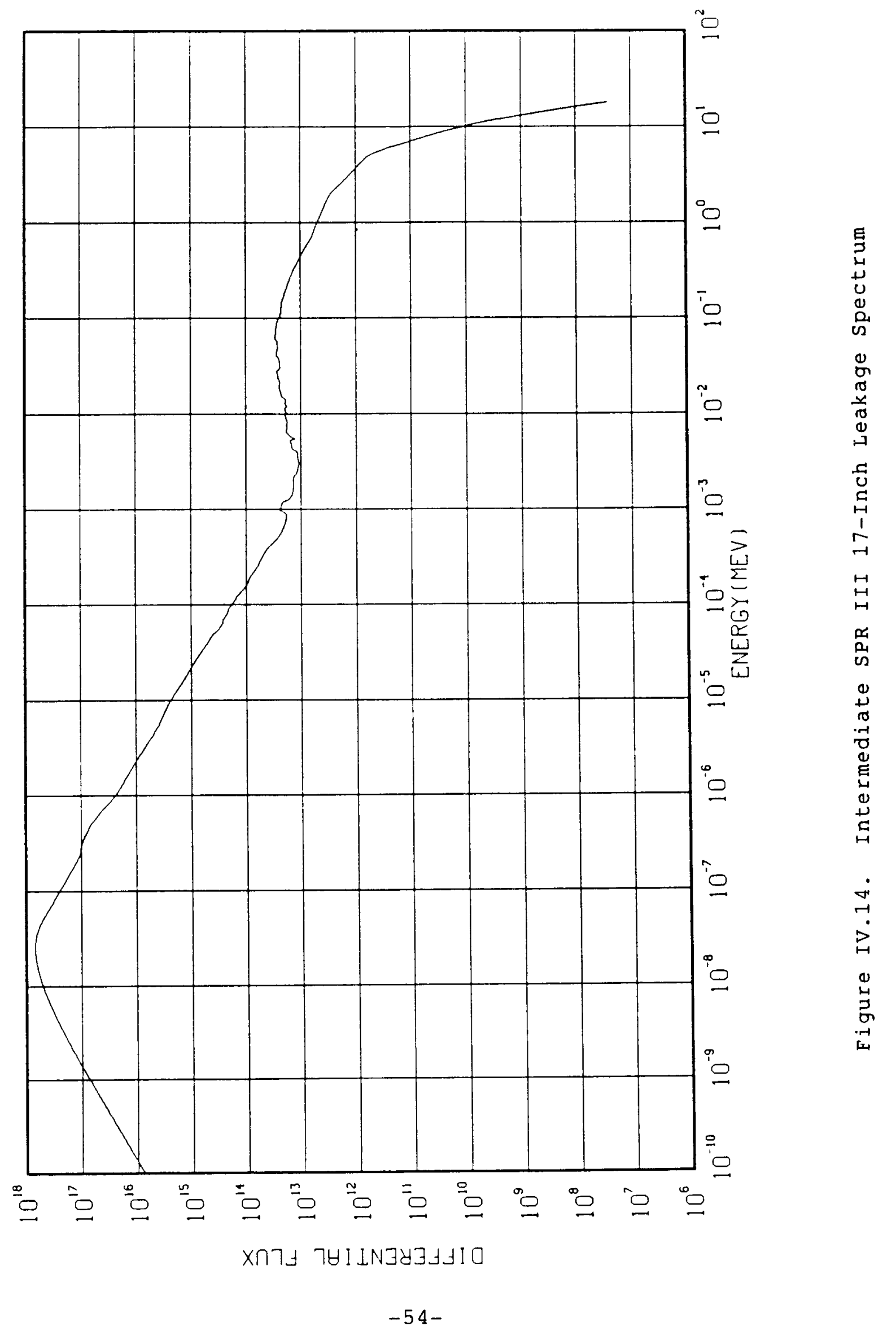




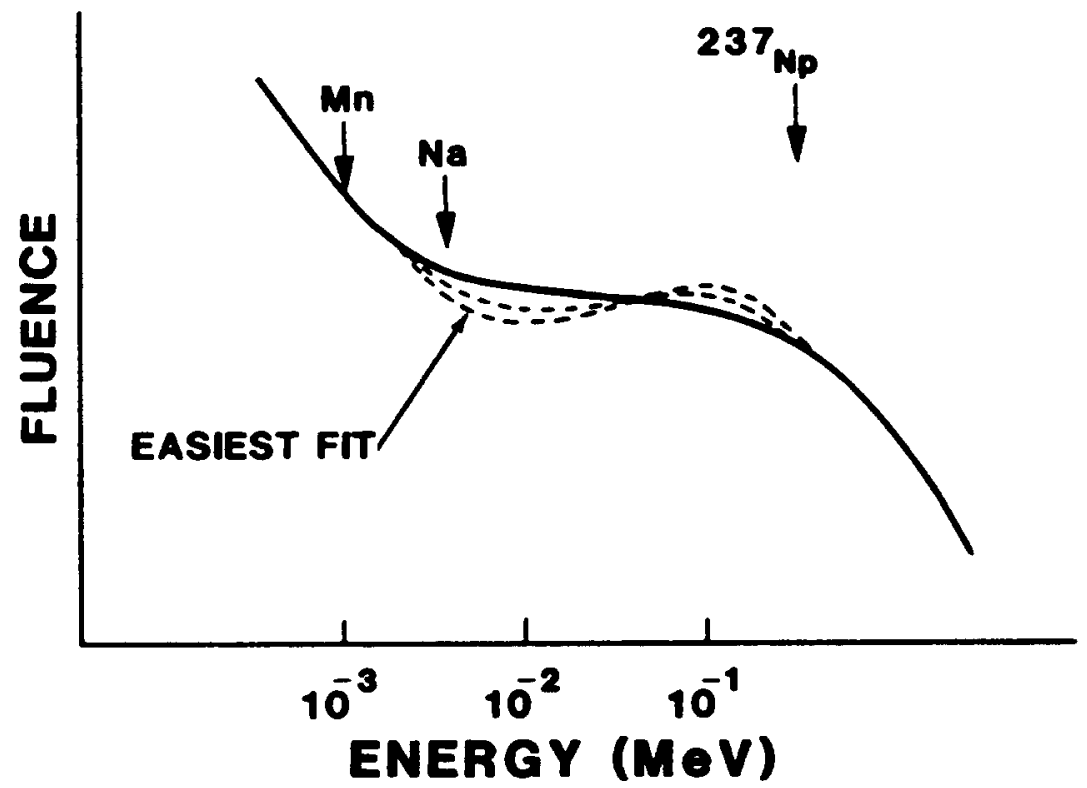

Figure IV.15. Increasingly Improved Fits With Increasing Dip at $1 \mathrm{E}-2 \mathrm{MeV}$

This result has been compared to that obtained from SANDII using a calculated trial spectrum. The spectrum shape for this configuration was calculated by the Monte Carlo code MORSE[10] by Ray Sartor after the work with an initial flat trial had been completed.[24] The MORSE result was compiled into the 27 energy groups listed in Table IV. 6 and plotted as a histogram in Figure IV.17. When this calculated spectrum was smoothed and used as a trial in SANDII, the spectrum of Figure IV.18 was obtained. A few smoothing operations provided a shape that matches the flat trial result almost exactly. The SI was 7.6. Similar procedures have been carried out successfully with other spectra. This suggests strongly that the final spectra obtained in this manner show little dependence on the form of the initial trial. The data set must be consistent, and the analyst must be experienced in dealing with the clues that SANDII provides and in applying a knowledge of the foil-set response functions. We also point out that, if a solution that requires minimization of curvature is applied without restraint, a reasonable solution that is flat in the dip region is obtained, but the $f$ it is not quite as good. In the region around $1 \mathrm{E}-2 \mathrm{MeV}$, where the foil responses are all low, it takes a large change in fluence to 


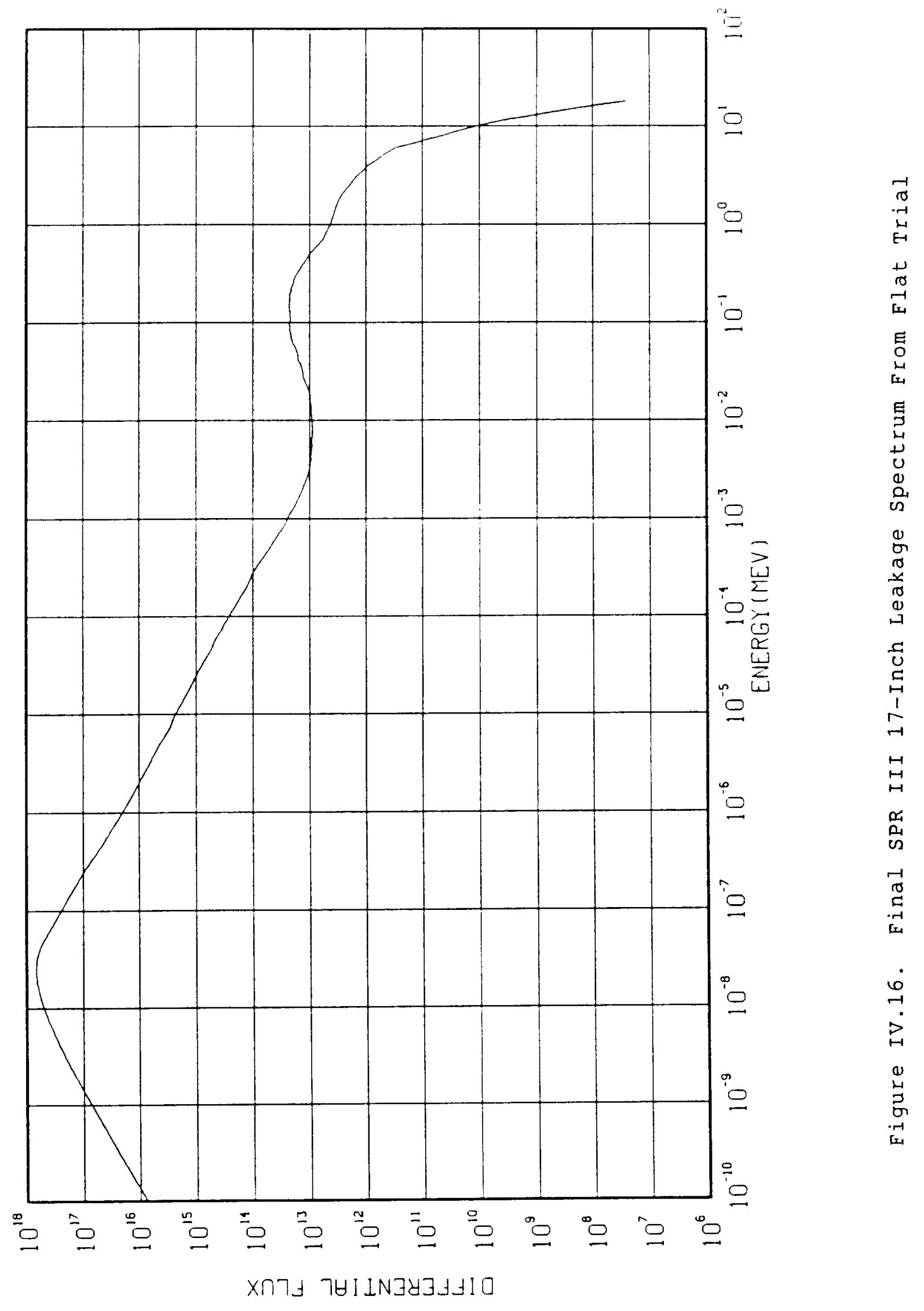


Table IV. 5

Reactions Used for SPR III 17-Inch Leakage spectrum

\begin{tabular}{|c|c|c|c|}
\hline $\begin{array}{l}\text { Reaction } \\
\text { and Cover }\end{array}$ & $\begin{array}{l}\text { Measured Activity } \\
\text { (Bq/nucleus) } \\
\text { (fissions/nucleus } \\
\text { for fission) }\end{array}$ & $\begin{array}{c}\text { Calculated Activity } \\
\int \sigma(e) \phi(E) d E\end{array}$ & $\begin{array}{l}\text { Deviation } \\
\text { (percent) }\end{array}$ \\
\hline $\mathrm{FE} 54 \mathrm{PE}+\mathrm{CD}$ & $2.486 \mathrm{E}-20$ & $2.471 \mathrm{E}-20$ & 0.61 \\
\hline$A U 197 G+C D$ & $1.094 \mathrm{E}-16$ & $1.095 E-16$ & -0.12 \\
\hline AU197G & 1. $275 E-16$ & 1. $274 \mathrm{E}-16$ & 0.12 \\
\hline FE56P +CD & $9.104 E-19$ & $9.101 \mathrm{E}-19$ & 0.03 \\
\hline$M N 55 G+C D$ & $3.490 \mathrm{E}-17$ & $3.490 \mathrm{E}-17$ & 0.00 \\
\hline$M G 24 P+C D$ & $2.067 E-19$ & $2.109 \mathrm{E}-17$ & -2.01 \\
\hline$A L 27 A+C D$ & $1.034 \mathrm{E}-19$ & $1.014 \mathrm{E}-19$ & 2.02 \\
\hline$N I 58 P+C D$ & 1. $402 \mathrm{E}-19$ & $1.407 \mathrm{E}-19$ & -0.36 \\
\hline$S 32 P$ & $4.680 \mathrm{E}-19$ & $4.705 E-19$ & -0.54 \\
\hline$N A 23 G+C D$ & $2.033 \mathrm{E}-19$ & $2.033 E-19$ & 0.00 \\
\hline $\mathrm{U} 235 \mathrm{~F}+\mathrm{CD}+\mathrm{B}$ & $2.326 \mathrm{E}-11^{+}$ & $2.330 E-11$ & -0.15 \\
\hline $\mathrm{U} 238 \mathrm{~F}+\mathrm{CD}+\mathrm{B}$ & $3.406 \mathrm{E}-12^{+}$ & $3.389 \mathrm{E}-12$ & 0.50 \\
\hline PU239F+CD+B & $3.085 E-11^{+}$ & $3.080 \mathrm{E}-11$ & 0.16 \\
\hline$N P 237 F+C D+B$ & $1.640 \mathrm{E}-11^{+}$ & $1.644 \mathrm{E}-11$ & -0.23 \\
\hline ZR902+CD & $2.476 \mathrm{E}-21$ & $2.477 \mathrm{E}-21$ & -0.02 \\
\hline
\end{tabular}



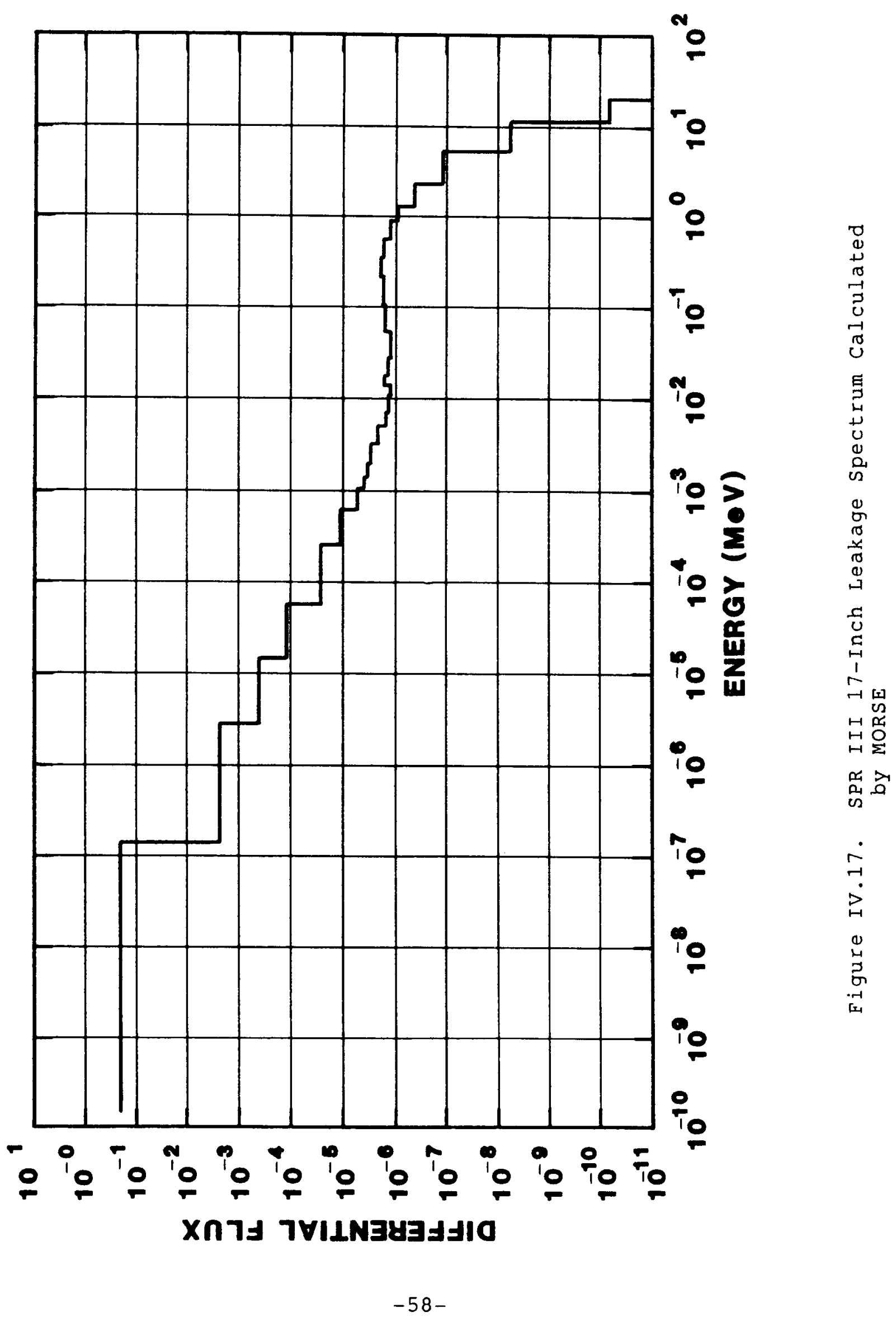


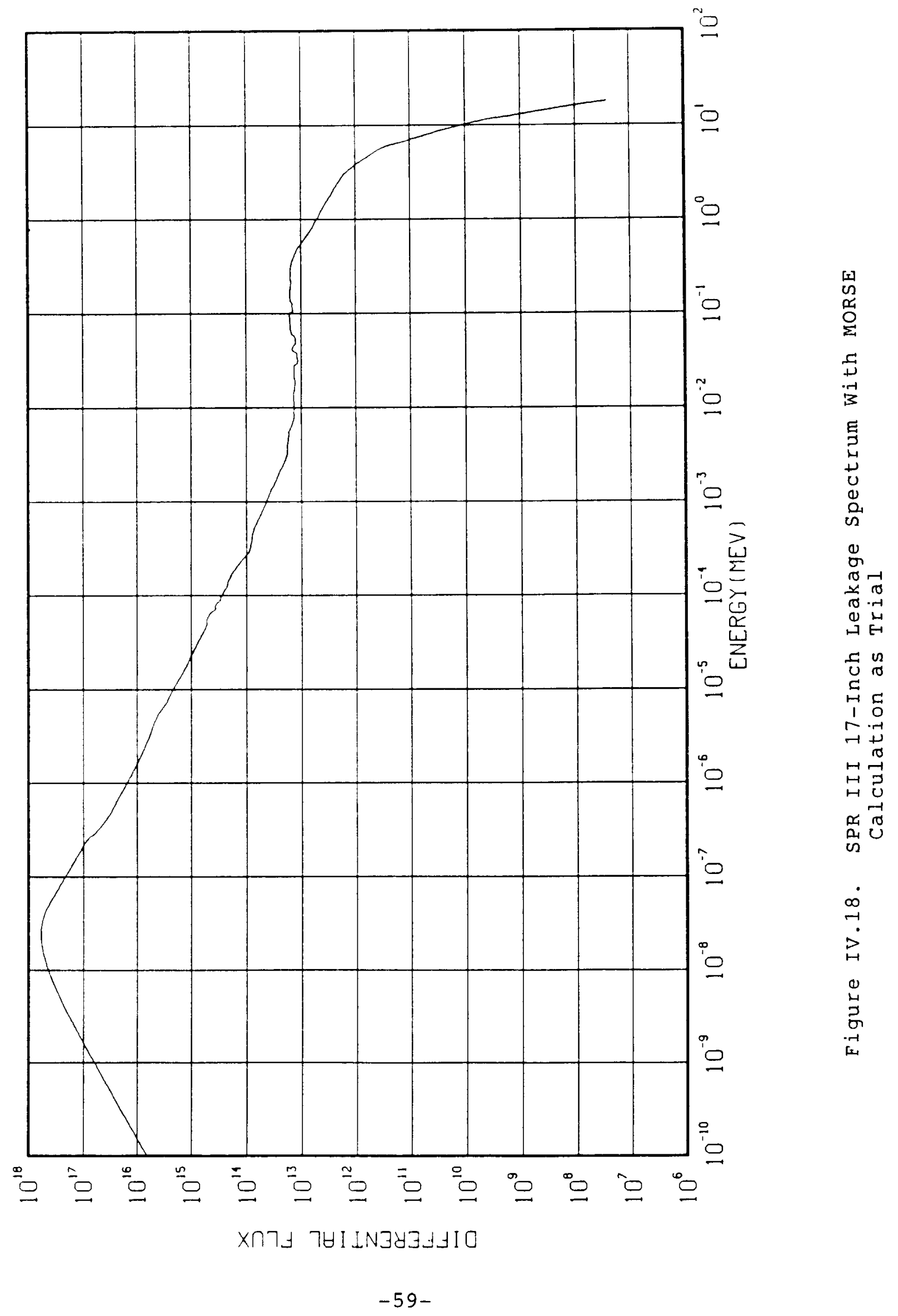


Table IV. 6

SPR III 17-Inch Leakage Spectrum Calculated by MORSE

\begin{tabular}{cccc}
$\begin{array}{c}\text { Energy Boundaries } \\
(\mathrm{MeV})\end{array}$ & Fluence & $\begin{array}{c}\text { Energy } \\
(\mathrm{MeV})\end{array}$ & Fluence \\
\hline $1.389 \mathrm{E}-10$ & $2.679 \mathrm{E}-1$ & $1.503 \mathrm{E}-2$ & $1.820 \mathrm{E}-6$ \\
$1.523 \mathrm{E}-7$ & $2.907 \mathrm{E}-3$ & $2.187 \mathrm{E}-2$ & $1.760 \mathrm{E}-6$ \\
$3.059 \mathrm{E}-6$ & $5.071 \mathrm{E}-4$ & $2.809 \mathrm{E}-2$ & $1.734 \mathrm{E}-6$ \\
$1.371 \mathrm{E}-5$ & $1.368 \mathrm{E}-4$ & $5.248 \mathrm{E}-2$ & $2.003 \mathrm{E}-6$ \\
$6.144 \mathrm{E}-5$ & $3.462 \mathrm{E}-5$ & $1.111 \mathrm{E}-1$ & $2.165 \mathrm{E}-6$ \\
$2.754 \mathrm{E}-4$ & $1.394 \mathrm{E}-5$ & $2.352 \mathrm{E}-1$ & $2.226 \mathrm{E}-6$ \\
$5.829 \mathrm{E}-4$ & $7.018 \mathrm{E}-6$ & $4.394 \mathrm{E}-1$ & $1.904 \mathrm{E}-6$ \\
$1.089 \mathrm{E}-3$ & $4.956 \mathrm{E}-6$ & $6.393 \mathrm{E}-1$ & $1.569 \mathrm{E}-6$ \\
$1.585 \mathrm{E}-3$ & $4.414 \mathrm{E}-6$ & $9.301 \mathrm{E}-1$ & $1.160 \mathrm{E}-6$ \\
$2.306 \mathrm{E}-3$ & $3.779 \mathrm{E}-6$ & 1.353 & $5.833 \mathrm{E}-7$ \\
$3.355 \mathrm{E}-3$ & $2.937 \mathrm{E}-6$ & 2.865 & $1.350 \mathrm{E}-7$ \\
$4.881 \mathrm{E}-3$ & $1.755 \mathrm{E}-6$ & 6.065 & $8.734 \mathrm{E}-9$ \\
$7.102 \mathrm{E}-3$ & $1.694 \mathrm{E}-6$ & $1.191 \mathrm{E}+1$ & $9.056 \mathrm{E}-11$ \\
$1.033 \mathrm{E}-2$ & $1.609 \mathrm{E}-6$ & $2.000 \mathrm{E}+1$ & \\
\hline
\end{tabular}


alter significantly the calculated activities. Therefore, one is justified in trying to find a spectrum that fits all activities to within the uncertainties of the measurements.

The speed with which the solutions described above were reached is illusory, because a number of mistakes had already been discovered and corrected, and care was taken to use a set of activities that were compatible. Most of the errors, the corrections, and the activity problems that are common to all of the spectrum measurements have already been discussed in the notes to Table IV.1. These include the deletion of the In115 activities because of self-shielding and gamma-ray excitation and the deletion of the nickel activity induced inside the boron ball.

The measured fission-foil activities listed in Table IV. 5 have been normalized by comparison of the nickel-foil activities obtained inside the boron ball and outside. This nickel activity ratio was compared to the average activity ratio for the other measured spectra. Then an adjustment in the fission-foil activities was made that would produce the average nickel activity ratio. This procedure is more fully discussed in Appendix $C$. John Meason at WSMR made similar corrections for the effect of the boron ball.[22]

\section{E. Using the Activity Option of SANDII}

Another option available with SANDII is very valuable when an analyst is becoming familiar with how the code approaches solutions. Given a spectrum and a list of reactions, the code will generate the activities that each reaction should acquire.

These calculated activities can then be used as input to the code with a trial of choice to see how well the adjustment operation is accomplished. There are three advantages: First, the actual spectrum is known; second, all activities at the outset must be consistent with it; third, there is no experimental uncertainty to disturb the approach to a solution. We illustrate this procedure using the spectrum for the activation run that was a result obtained from a prior measurement at the WSMR FBR. The trial function was flat $(\phi=1.0$ at each energy). The approach to a solution began in the same manner as described above, but at first the NA23G reaction proved very difficult to fit from any trial that was devised by observation of the adjustment process. Its activity was obviously low. Yet this activity could not be discarded, because it was a priori valid. The code depressed the spectrum wherever the sodium response was dominant (as we pointed out in Figure IV.14). We decided that, in an experimental situation, one would trust the gold and manganese activities and force the trial to follow a straight line 
through the principal resonance peaks of these reactions. With this constraint on the lower energy region, the sodium activity could be altered only by changing the spectrum in the $3 \mathrm{E}-3$ to $0.5 \mathrm{MeV}$ region. Increasingly improved fits were obtained by allowing the dip near $1 \mathrm{E}-2 \mathrm{MeV}$ and the hump near 0.1 MeV to develop. This took place slowly over many iterations, because this structure progressed only slightly with each run before the activity was also compensated for at other energies. Finally, a result was obtained that was very close to the activity run spectrum.

The SPR III 17-inch leakage spectrum adjustment process discussed in this chapter exhibited similar characteristics. No fit to the sodium activity could be found. That reaction was initially left out of the analysis, and a spectrum fairly flat through the $1 \mathrm{E}-3$ to $0.5 \mathrm{MeV}$ region was found to be compatible. The presumption was that the sodium, sensitive in the thermal region, was suffering from self-shielding. Measurements were made with a bare salt tablet and one encased in a cadmium box. The ratio of the measured activities (bare to cadmium-covered) was 3. An activity run with SANDI, without and with a cover, showed that the ratio should indeed be 3 without any correction for self-shielding. Clearly, the selfshielding argument was not sufficient justification for deleting the reaction.

The result was that, by applying the procedure just discussed (constraining the solution to fit smoothly the gold and manganese foils), the wavy form of the solution began to emerge, and the SD of the solutions continued to drop. Since that time, better fits to other spectra have accrued from application of the same procedure. The evidence is that this feature is real in some of these spectra, although it is not of great importance when damage functions in silicon are being determined.

The methods for finding appropriate spectra with SANDII indicate that well-defined solutions can be found that have very little dependence on the trial function used to initiate the adjustment process. This also means that library or calculated trials may be used to shorten the adjustment time, so long as care is taken to recognize when the trial is not appropriate in some important way that generates response functions that are grossly in error. Finer features of the spectra can be revealed if the foil data are accurate and cover the energy range of interest well. In addition, the code itself is a very important tool for finding errors and discrepancies in the data set.

Finally, because the final spectra exhibit little dependence upon the initial trial, the procedure goes far beyond that which can be considered strictly an adjustment method. 
As mentioned in the introduction, spectra in a variety of configurations at three different reactor facilities have been determined by the method described in chapter IV. In the first section of this chapter, each of these spectra will be discussed. It is evident that each adjustment operation requires individual attention. The analysis of all of them was initiated with a flat trial spectrum so that the differences that develop are, in fact, caused by the differences in the foil sets and not by differences in the trials. Far greater attention has been devoted to certain cases whose spectra are more important or more difficult.

The second section includes compilations and comparisons of the spectral characteristics that were obtained. The comparisons reveal interesting consistencies common to groups of spectra that might show additional real structure, problems with certain cross sections, or errors in the laboratory's determination of activities and fluences.

In section $C$, determination of spectrum-averaged cross sections, activities, and fluences are discussed, together with calibrations and corrections recently made at SNLA to these parameters.

Section D explains how the spectrum measurements are related to the calculation of damage in silicon devices and exhibits the necessary connections between the damage and the fluence levels measured with sulfur dosimetry systems. In Section E, comparisons with other measured spectra are made, and in section $F$, some of the effects of error in the measurements on integral quantities are discussed briefly.

Because of the difficulty in establishing that foils placed inside the boron ball (mainly the fission foils) are exposed to the same fluence as are those in the rest of the foil set, all fission-foil activities have been normalized so that a certain measured ratio of nickel foils--bare and shielded by the boron ball--is maintained. Appendix $C$ explains how this was done and provides the means by which an analyst can recover the measured fission-foil activities. 


\section{A. Spectra Measurements}

The spectra and the spectral characteristics are grouped by reactor facility: First, the SPR III spectra and characteristics are presented; second, those taken from the WSMR FBR; and third, the ACRR spectra. Included with the spectrum tables are the gamma-ray absorbed doses observed for each exposure. 


\section{(1) SPR III Central Cavity in Free Field (BARNOB6)}

The mechanical and operational characteristics of the reactor are discussed thoroughly in the SPR III experimenter's manual.[9] SPR III is a fast-pulse reactor of the Godiva family and has a $17.8-\mathrm{cm}$ diameter central cavity for experiments.

In Chapter IV, the development of a spectrum for the SPR III bare cavity was discussed in detail. The fission foils for that experiment were mounted in a boron ball at the center of the cavity, and corrections had to be made to the fission-foil activities because they were exposed to a lower fluence than were the rest of the foils positioned at an axial radius of $5.7 \mathrm{~cm}$. The correction was made by adjusting the activities of the foils so that the ratio of the nickel activity inside the ball to that outside the ball was 0.894 . This number is an average of the nickel ratios for the other spectrum measurements, as explained in Appendix C. However, this correction does not take into consideration that the cross-section set used by SANDII does not properly account for the attenuation of neutrons by the ball. Therefore, for this exposure, the fission foils were exposed without the boron ball cover at the same radius as the other foils. Since the ball no longer shadowed the other foils, all of them were exposed during the same reactor run. Thus, no normalizations for ball or relative fluence levels between runs were needed. The fission-foil sensitivities were still concentrated above 1E-2 MeV because the thermal tail is small.

The reactions are listed in Table V.1 (in SANDII notation), together with cover type and thickness and the activities. Those reactions marked with an asterisk were not used in the determination of the spectrum, for the same reasons discussed in Section A of Chapter IV.

Table V.2 lists 50 of the 621 differential and integral fluence values determined by SANDII as a function of energy. In all cases the values were normalized by adjusting the integral fluence above $10 \mathrm{keV}$ to unity (1.0). SANDII was instructed to add a thermal tail to the trial below $5 \mathrm{E}-8 \mathrm{MeV}$ and a fission high-energy tail above $8 \mathrm{MeV}$. The spectral characteristics are listed with the table. The code was allowed to iterate until the difference between SDs of measured and calculated activities was less than $2.5 \%$. Figure V.1 is a plot of the spectrum.

The thermal tail is required for consistency with the gold, manganese, and sodium activities, but it accounts for only $0.1 \%$ of the total fluence. The SI of 8.45 is $1 \%$ higher than that previously used in this laboratory for the bare cavity. 
Table V.1

Foil Activities From SPR II in Cavity Free Field

\begin{tabular}{|c|c|c|c|c|}
\hline Reaction & Cover & : (atoms & parn) & $\begin{array}{c}\text { Activity } \\
\text { (Bq/nucleus) }\end{array}$ \\
\hline $\begin{array}{l}\text { AU197G } \\
\text { AU197G } \\
\text { MN55G } \\
\text { MG24P } \\
\text { FE56P } \\
\text { FE54P } \\
\text { AL27A } \\
\text { NI58P } \\
\text { NA23G } \\
\text { ZR902 } \\
\text { S32P } \\
\text { *IN115G } \\
\text { *IN115N } \\
\text { *NI58P }\end{array}$ & $\begin{array}{c}2.587 E-3 \\
- \\
2.587 E-3 \\
2.587 E-3 \\
2.587 E-3 \\
2.587 E-3 \\
2.587 E-3 \\
2.587 E-3 \\
4.705 E-3 \\
2.587 E-3 \\
- \\
2.587 E-3 \\
2.587 E-3 \\
4.705 E-3\end{array}$ & $\begin{array}{l}\text { cadmium } \\
- \\
\text { cadmium } \\
\text { cadmium } \\
\text { cadmium } \\
\text { cadmium } \\
\text { cadmium } \\
\text { cadmium } \\
\text { cadmium } \\
\text { cadmium } \\
- \\
\text { cadmium } \\
\text { cadmium } \\
\text { cadmium }\end{array}$ & 0.101 boron & $\begin{array}{r}1.480 \mathrm{E}-16 \\
1.515 \mathrm{E}-16 \\
1.261 \mathrm{E}-16 \\
2.998 \mathrm{E}-18 \\
1.276 \mathrm{E}-17 \\
3.294 \mathrm{E}-19 \\
1.431 \mathrm{E}-18 \\
2.010 \mathrm{E}-18 \\
1.052 \mathrm{E}-18 \\
4.157 \mathrm{E}-20 \\
6.078 \mathrm{E}-18 \\
1.061 \mathrm{E}-14 \\
1.507 \mathrm{E}-15 \\
1.924 \mathrm{E}-18 \\
\text { Activity } \\
\text { (Eissions/nucleus) }\end{array}$ \\
\hline $\begin{array}{l}\text { U235F } \\
\text { U2 } 38 \mathrm{~F} \\
\text { PU239F } \\
\text { NP2 } 37 \mathrm{~F}\end{array}$ & $\begin{array}{l}4.705 E-3 \\
4.705 E-3 \\
4.705 E-3 \\
4.705 E-3\end{array}$ & $\begin{array}{l}\text { cadmium } \\
\text { cadmium } \\
\text { cadmium } \\
\text { cadmium }\end{array}$ & $\begin{array}{l}0.101 \text { boron } \\
0.101 \text { boron } \\
0.101 \text { boron } \\
0.101 \text { boron }\end{array}$ & $\begin{array}{l}4.050 \mathrm{E}-10 \\
5.279 \mathrm{E}-11 \\
4.994 \mathrm{E}-10 \\
2.577 \mathrm{E}-10\end{array}$ \\
\hline
\end{tabular}


Table V.2

SPR III Bare Cavity spectrum

$E(\mathrm{MeV})$ Differential Integral $\mathrm{E}(\mathrm{MeV})$ Differential Integral

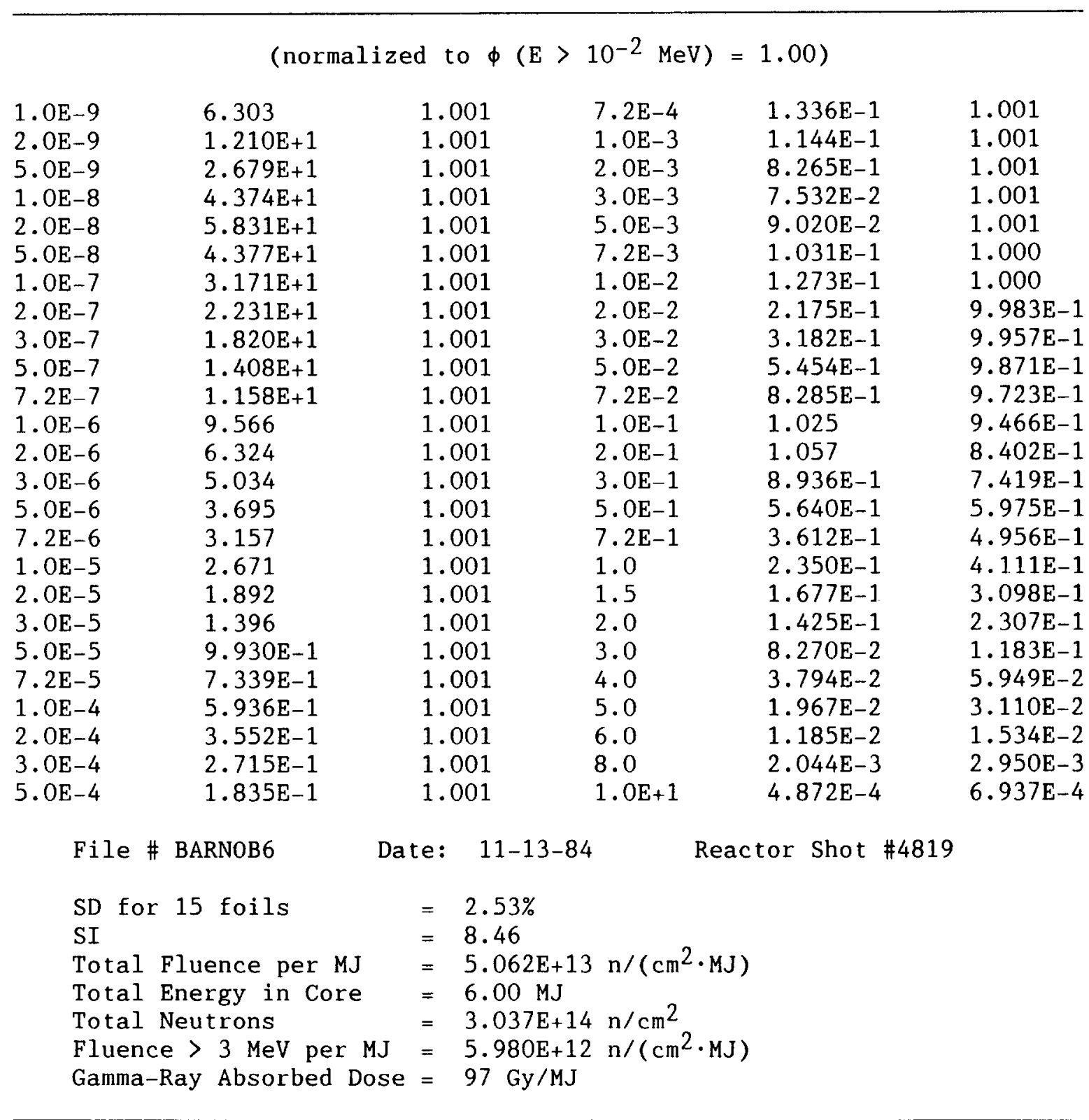




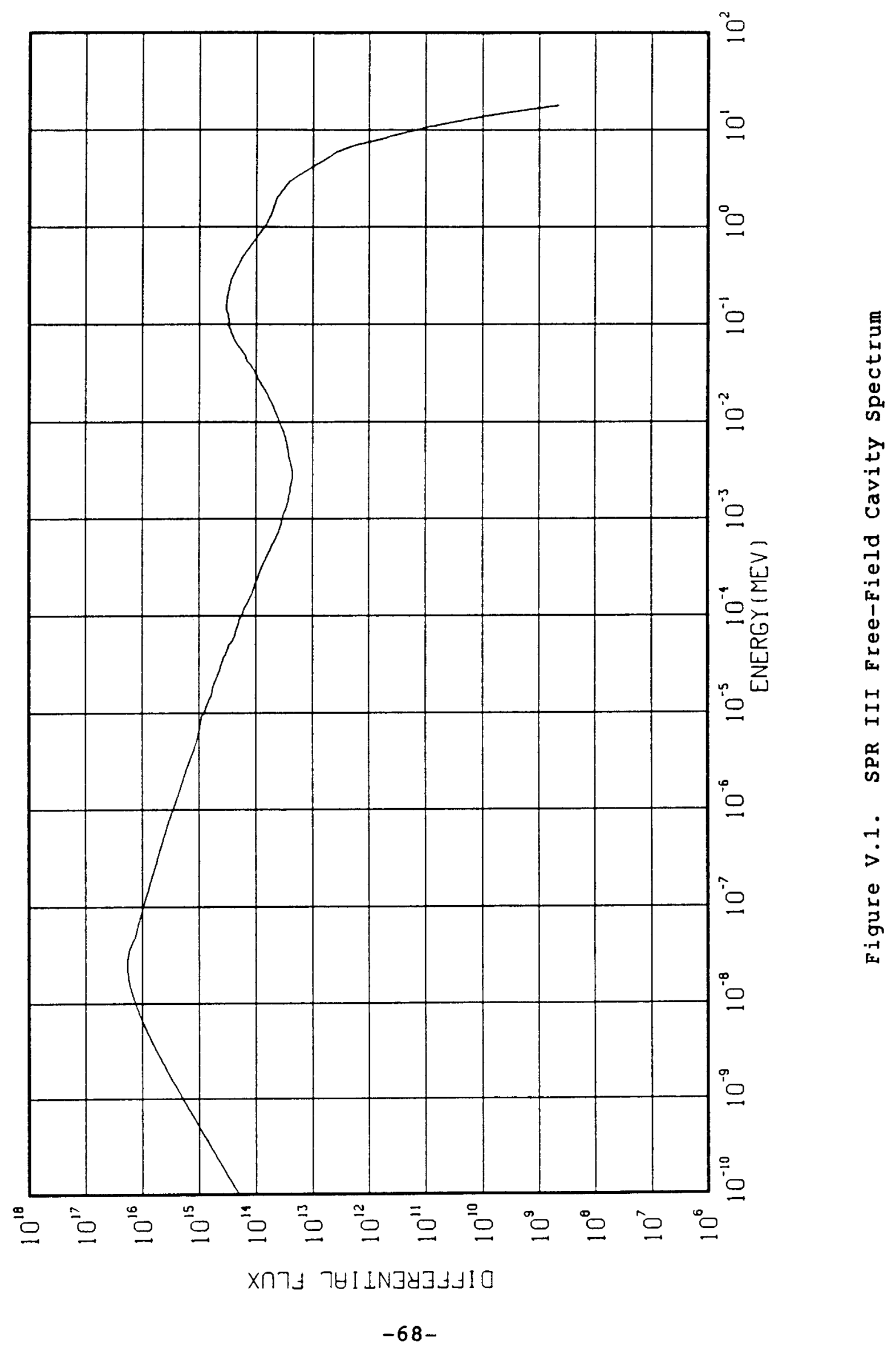


(2) SPR III Central Cavity with Polyethylene Liner (POLYCA48)

For this experiment, a $2.54-\mathrm{cm}$ thick cylindrical polyethylene box with end caps was placed inside the boron carbide liner. The dimensions of the box are shown in Figure V.2. The space available for the foil set was smaller than usual here, and the shadowing effect of the boron ball on the foils with low-energy responses was very evident ( $230 \%$ on gold). In this case, separate runs for the boron ball foils and for the other foils were mandatory. High-threshold-energy foils such as sulfur and iron, however, exhibited no significant differences in activity after being exposed in the same steady-state run $(300 \mathrm{~s}$ at $7 \mathrm{~kW}=2.1 \mathrm{MJ})$, with or without the presence of the boron ball. Therefore, it has been assumed that the fast fluence was not altered by the boron ball.

Because the foils were much closer to the vertical centerline than in the bare cavity case, no correction for any radial profile in the fluence was applied to the data. Sodium was not included in this foil set, because at the time this experiment was run, no confidence had been developed in the use of this reaction, and space was limited.

Self-shielding was a problem for the pure gold foils used in these early experiments, so these foils were sandwiched between gold foils to try to reduce the effects of selfshielding in the primary foil. This sandwich method was not very accurate, but it was the only option available at the time. The outer gold foil thicknesses were then entered in SANDII, which corrects for the cover by assuming exponential attenuation. In all other spectra, this correction was unnecessary because dilute gold foils were used. The activities are listed in Table V.3, and the spectrum is listed in Table V.4.

The NP237F foil was not available for this test. This foil is sensitive in the $0.5-\mathrm{MeV}$ region and usually constrains the spectrum so that the tendency of the $\ln 115\left(\mathrm{n}, \mathrm{n}^{\prime}\right)$ In 115 reaction to appear high becomes evident. In this case, the indium was the only foil available in the $1-M e v$ region. Although the IN115N reaction was still not used in the analysis presented here, it was found that when it was included, the SI was changed by only $1 \%$. A trial run with a $15 \%$ reduction in the IN115N activity led to a reduction of $1 \%$ in the SI.

The code iterated until the SD fell below $3 \%$, at which point progress became slow and the procedure was terminated. The spectrum is plotted in Figure V. 3 . 


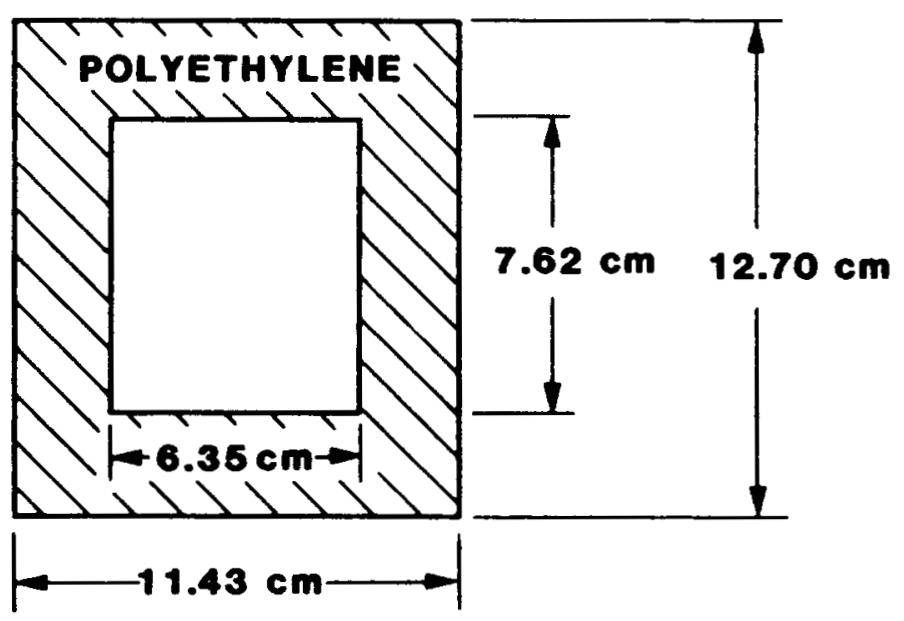

Figure V.2. Cavity Polyethylene Liner Dimensions 
Table V. 3

Foil Activities From Polyethylene-Lined SPR III Cavity

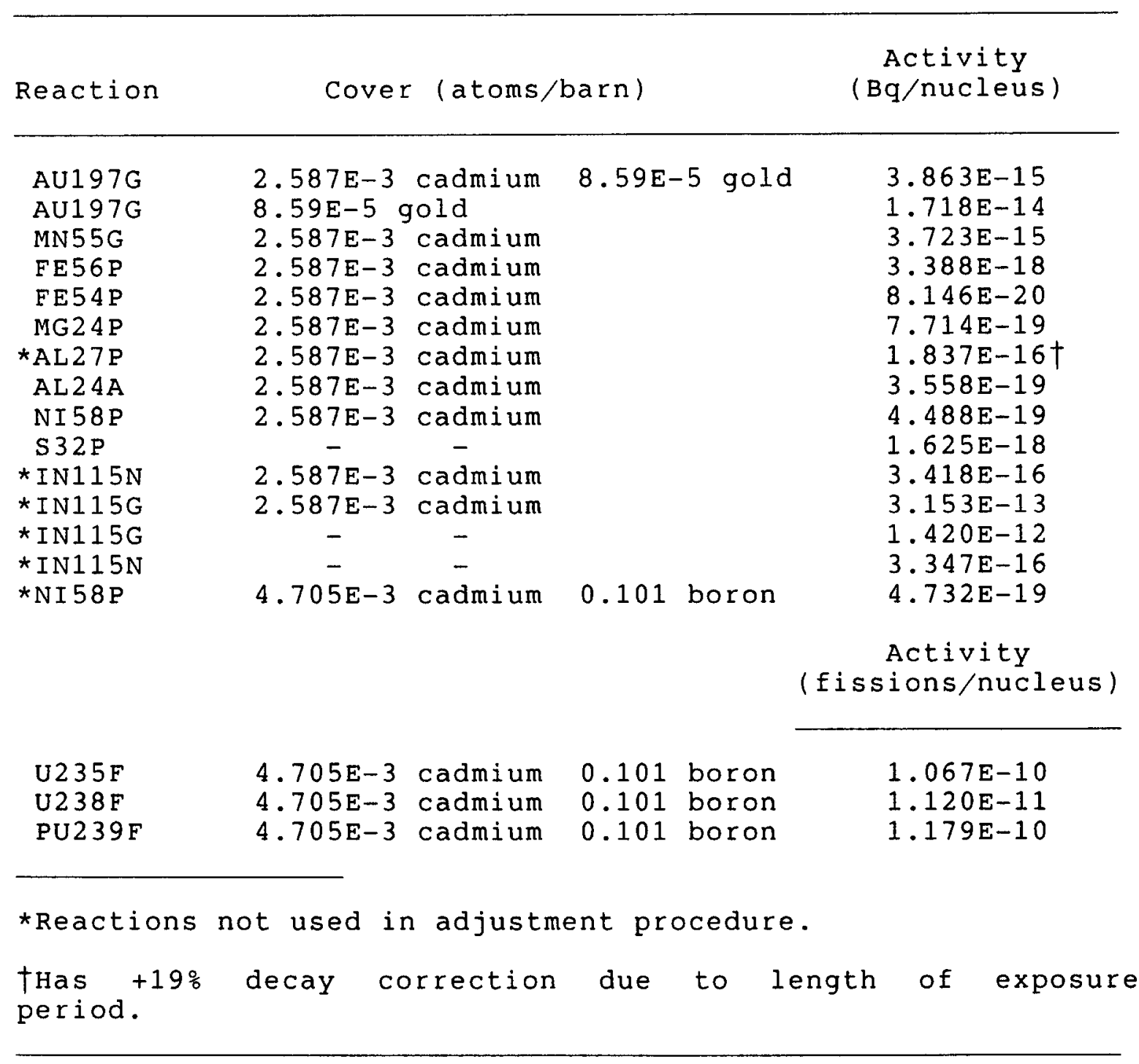


Table V. 4

SPR III Central Cavity Spectrum with Polyethylene Liner

\begin{tabular}{llllll}
\hline E (MeV) & Differential & Integral & $\mathrm{E}(\mathrm{MeV})$ & Differential & Integral \\
& & & & & \\
$1.0 \mathrm{E}-9$ & $9.997 \mathrm{E}+5$ & 2.250 & $7.2 \mathrm{E}-4$ & $8.072 \mathrm{E}+1$ & 1.179 \\
$2.0 \mathrm{E}-9$ & $1.920 \mathrm{E}+6$ & 2.248 & $1.0 \mathrm{E}-3$ & $6.068 \mathrm{E}+1$ & 1.159 \\
$5.0 \mathrm{E}-9$ & $4.249 \mathrm{E}+6$ & 2.239 & $2.0 \mathrm{E}-3$ & $3.173 \mathrm{E}+1$ & 1.115 \\
$1.0 \mathrm{E}-8$ & $6.937 \mathrm{E}+6$ & 2.211 & $3.0 \mathrm{E}-3$ & $2.191 \mathrm{E}+1$ & 1.088 \\
$2.0 \mathrm{E}-8$ & $9.241 \mathrm{E}+6$ & 2.128 & $5.0 \mathrm{E}-3$ & $1.434 \mathrm{E}+1$ & 1.052 \\
$5.0 \mathrm{E}-8$ & $6.727 \mathrm{E}+6$ & 1.869 & $7.2 \mathrm{E}-3$ & $1.005 \mathrm{E}+1$ & 1.025 \\
$1.0 \mathrm{E}-7$ & $2.315 \mathrm{E}+6$ & 1.671 & $1.0 \mathrm{E}-2$ & 7.901 & 1.000 \\
$2.0 \mathrm{E}-7$ & $7.539 \mathrm{E}+5$ & 1.535 & $2.0 \mathrm{E}-2$ & 4.207 & $9.423 \mathrm{E}-1$ \\
$3.0 \mathrm{E}-7$ & $3.693 \mathrm{E}+5$ & 1.480 & $3.0 \mathrm{E}-2$ & 3.054 & $9.059 \mathrm{E}-1$ \\
$5.0 \mathrm{E}-7$ & $1.465 \mathrm{E}+5$ & 1.431 & $5.0 \mathrm{E}-2$ & 2.041 & $8.553 \mathrm{E}-1$ \\
$7.2 \mathrm{E}-7$ & $7.261 \mathrm{E}+4$ & 1.407 & $7.2 \mathrm{E}-2$ & 1.508 & $8.163 \mathrm{E}-1$ \\
$1.0 \mathrm{E}-6$ & $4.144 \mathrm{E}+4$ & 1.391 & $1.0 \mathrm{E}-1$ & 1.224 & $7.777 \mathrm{E}-1$ \\
$2.0 \mathrm{E}-6$ & $1.396 \mathrm{E}+4$ & 1.367 & $2.0 \mathrm{E}-1$ & $7.225 \mathrm{E}-1$ & $6.836 \mathrm{E}-1$ \\
$3.0 \mathrm{E}-6$ & $7.996 \mathrm{E}+3$ & 1.356 & $3.0 \mathrm{E}-1$ & $5.251 \mathrm{E}-1$ & $6.211 \mathrm{E}-1$ \\
$5.0 \mathrm{E}-6$ & $4.292 \mathrm{E}+3$ & 1.344 & $5.0 \mathrm{E}-1$ & $3.653 \mathrm{E}-1$ & $5.327 \mathrm{E}-1$ \\
$7.2 \mathrm{E}-6$ & $2.729 \mathrm{E}+3$ & 1.336 & $7.2 \mathrm{E}-1$ & $2.876 \mathrm{E}-1$ & $4.605 \mathrm{E}-1$ \\
$1.0 \mathrm{E}-5$ & $1.898 \mathrm{E}+3$ & 1.330 & 1.0 & $2.255 \mathrm{E}-1$ & $3.873 \mathrm{E}-1$ \\
$2.0 \mathrm{E}-5$ & $1.044 \mathrm{E}+3$ & 1.316 & 1.5 & $1.590 \mathrm{E}-1$ & $2.896 \mathrm{E}-1$ \\
$3.0 \mathrm{E}-5$ & $7.611 \mathrm{E}+2$ & 1.307 & 2.0 & $1.250 \mathrm{E}-1$ & $2.175 \mathrm{E}-1$ \\
$5.0 \mathrm{E}-5$ & $5.307 \mathrm{E}+2$ & 1.294 & 3.0 & $7.255 \mathrm{E}-2$ & $1.192 \mathrm{E}-1$ \\
$7.2 \mathrm{E}-5$ & $4.102 \mathrm{E}+2$ & 1.283 & 4.0 & $3.742 \mathrm{E}-2$ & $6.521 \mathrm{E}-2$ \\
$1.0 \mathrm{E}-4$ & $3.251 \mathrm{E}+2$ & 1.273 & 5.0 & $2.264 \mathrm{E}-2$ & $3.525 \mathrm{E}-2$ \\
$2.0 \mathrm{E}-4$ & $2.070 \mathrm{E}+2$ & 1.247 & 6.0 & $1.430 \mathrm{E}-2$ & $1.652 \mathrm{E}-2$ \\
$3.0 \mathrm{E}-4$ & $1.587 \mathrm{E}+2$ & 1.228 & 8.0 & $2.318 \mathrm{E}-3$ & $3.303 \mathrm{E}-3$ \\
$5.0 \mathrm{E}-4$ & $1.163 \mathrm{E}+2$ & 1.201 & 10.0 & $5.427 \mathrm{E}-4$ & $7.685 \mathrm{E}-4$
\end{tabular}

File \# POLYCA48

Date: 5-21-84

Reactor Shot \#3037

$\begin{array}{ll}\text { SD for } 13 \text { foils } & =2.46 \% \\ \text { SI } & =8.39 \\ \text { Total Fluence per MJ } & =7.695 \mathrm{E}+13 \mathrm{n} /\left(\mathrm{cm}^{2} \cdot \mathrm{MJ}\right) \\ \text { Total Energy in Core } & =2.1 \mathrm{MJ} \\ \text { Total Neutrons } & =1.616 \mathrm{E}+14 \mathrm{n} / \mathrm{cm}^{2} \\ \text { Fluence }>3 \mathrm{MeV} \text { per MJ } & =4.077 \mathrm{E}+12 \mathrm{n} /\left(\mathrm{cm}^{2} \cdot \mathrm{MJ}\right) \\ \text { Gamma-Ray Absorbed Dose } & =191 \mathrm{~Gy} / \mathrm{MJ}\end{array}$ 


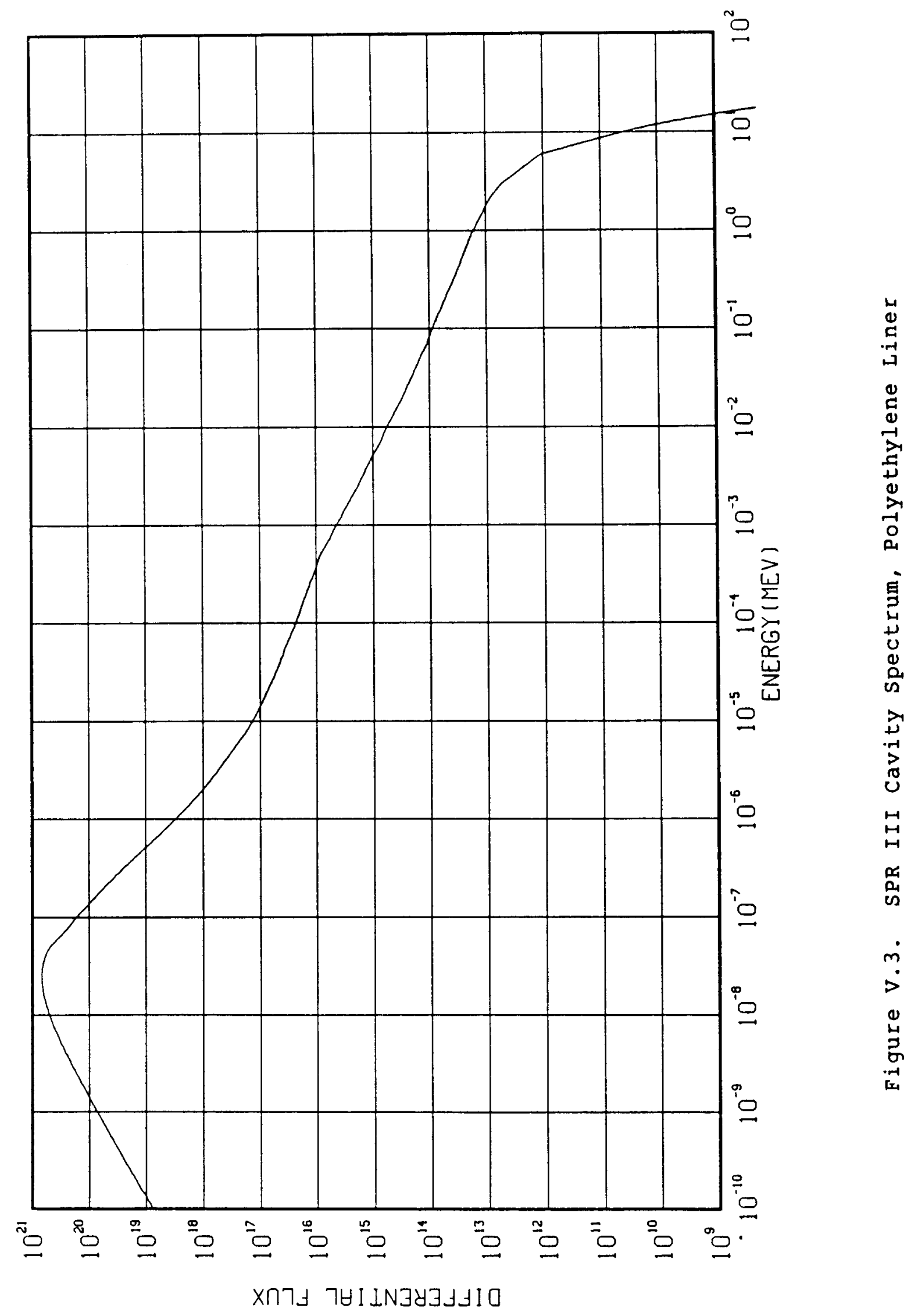




\section{(3) SPR II Cavity With Aluminum Liner (RAN19)}

Figure V.4a is a drawing of a thick aluminum liner incorporated in this cavity measurement. The inner cavity was so small in this case that room was unavailable for an axial flux profile measurement with nickel or sulfur foils. In the run without the boron ball in place (containing the fission foils), the foils were arranged on the inner surface of the aluminum cylinder in two rows, as shown in Figure V.4b. The gold foil \#2 and the sulfur pellet had no cadmium cover. The other foils used in the analysis were enclosed in cadmium. The foils sensitive at low energies were placed on the upper ring and may have been exposed to a slightly lower fluence. It is unlikely, however, that the fluence above $10 \mathrm{E}-2 \mathrm{keV}$, where the SI and silicon damage are determined, were significantly altered by a slight reduction in the gold activity.

The 16 foils are listed in Table V.5, and the spectrum is listed in Table V.6. The iteration procedure was stopped when the SD reached $2.8 \%$. There is some conflict between the manganese and sodium reactions in this case, which has not been resolved by the choice of the trial used here. This caused the slightly bumpy structure of the cross sections to appear between $10 \mathrm{E}-4$ and $3 \mathrm{E}-1$ MeV. Disagreement among foils at higher energies, where the reactions are mostly of the threshold type without resonance structure, do not usually lead to spectra with oscillations at the high energies.

The large mass of aluminum $(\sim 22 \mathrm{~kg})$ in the core modified the SI by more than expected (from 8.5 to 10.4). The extra scattering has caused a partial filling in of the dip at 1E-2 MeV that was present in the bare cavity spectrum. The spectrum is shown in Figure V.5. 


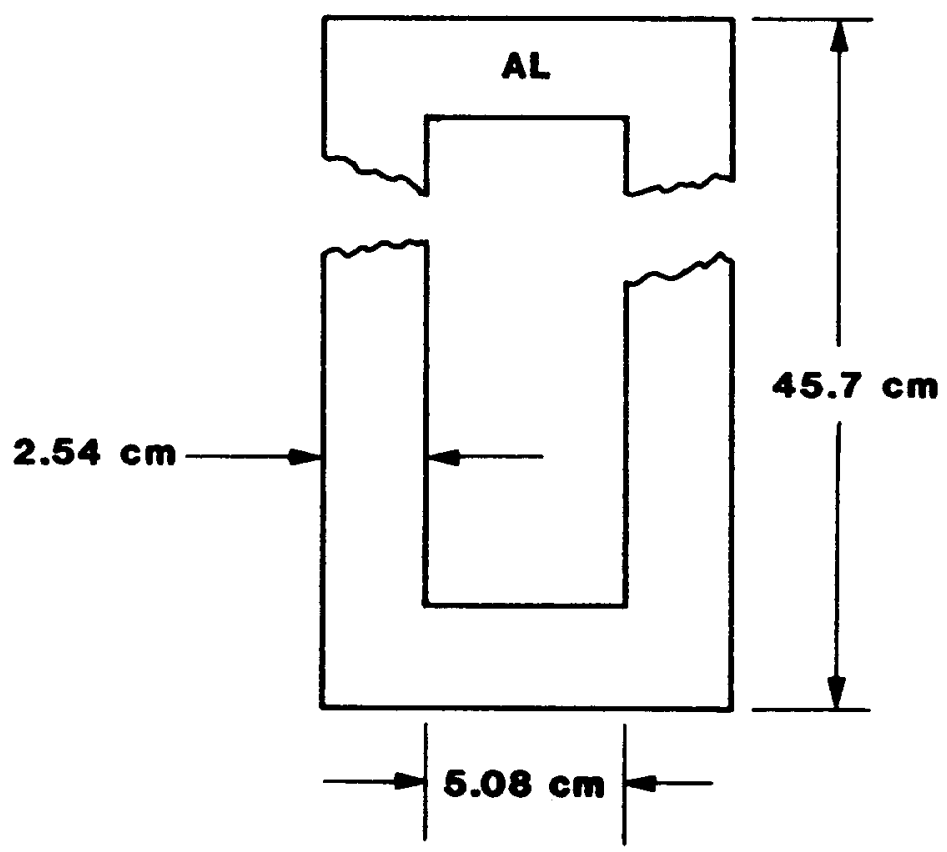

a. Aluminum Liner Geometry

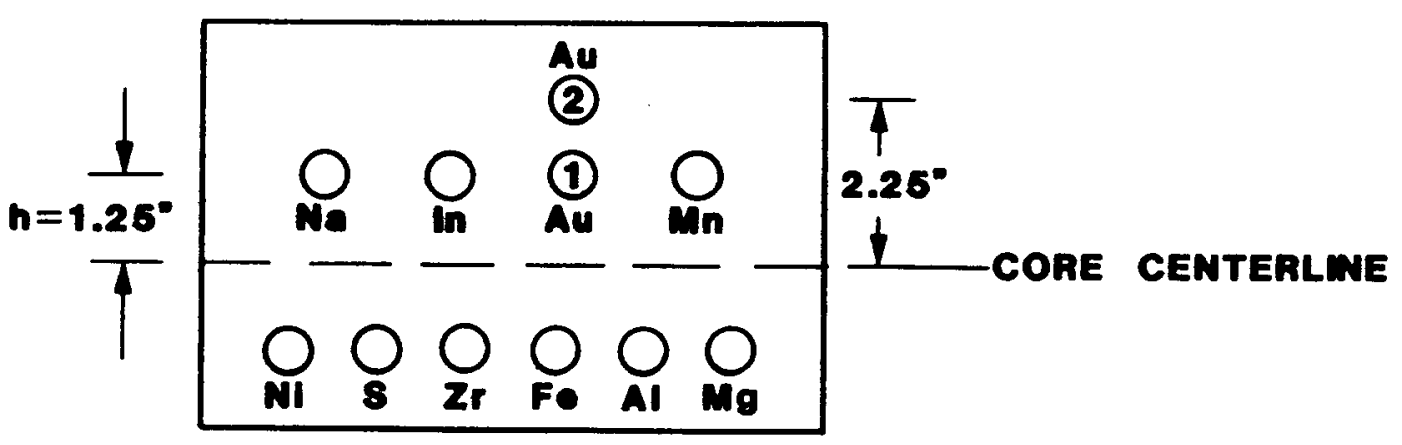

b. Foll arrengement on the Cylindrical Liner Inner Surfece

Figure V.4. Dimensions of Cylindrical Aluminum Liner and Foil Arrangement 
Table V.5

Foil Activities From SPR III Cavity With Aluminum Liner

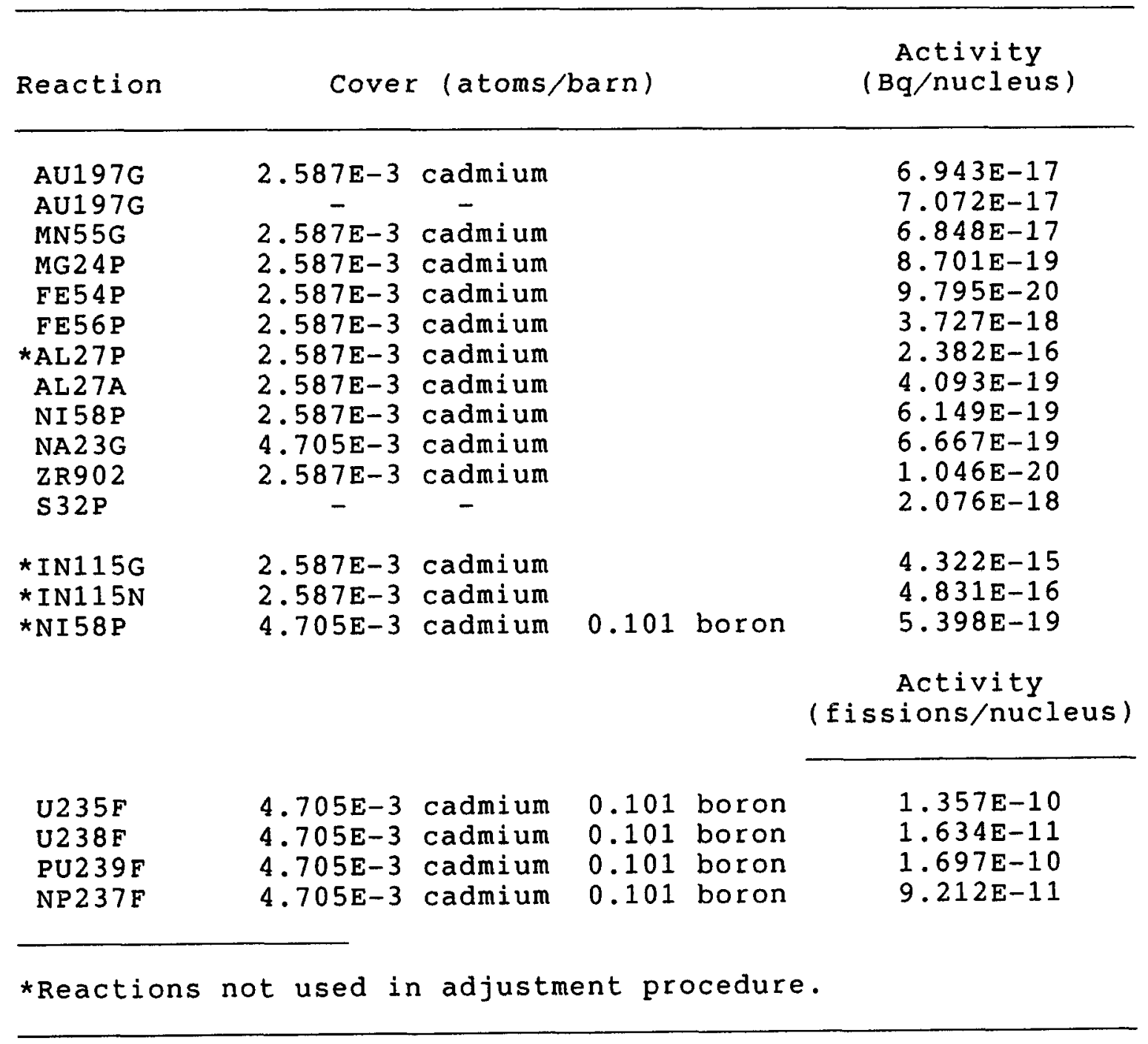


Table V.6

SPR III Central Cavity Spectrum With Aluminum Liner

$E(\mathrm{MeV})$ Differential Integral $\mathrm{E}(\mathrm{MeV})$ Differential Integral

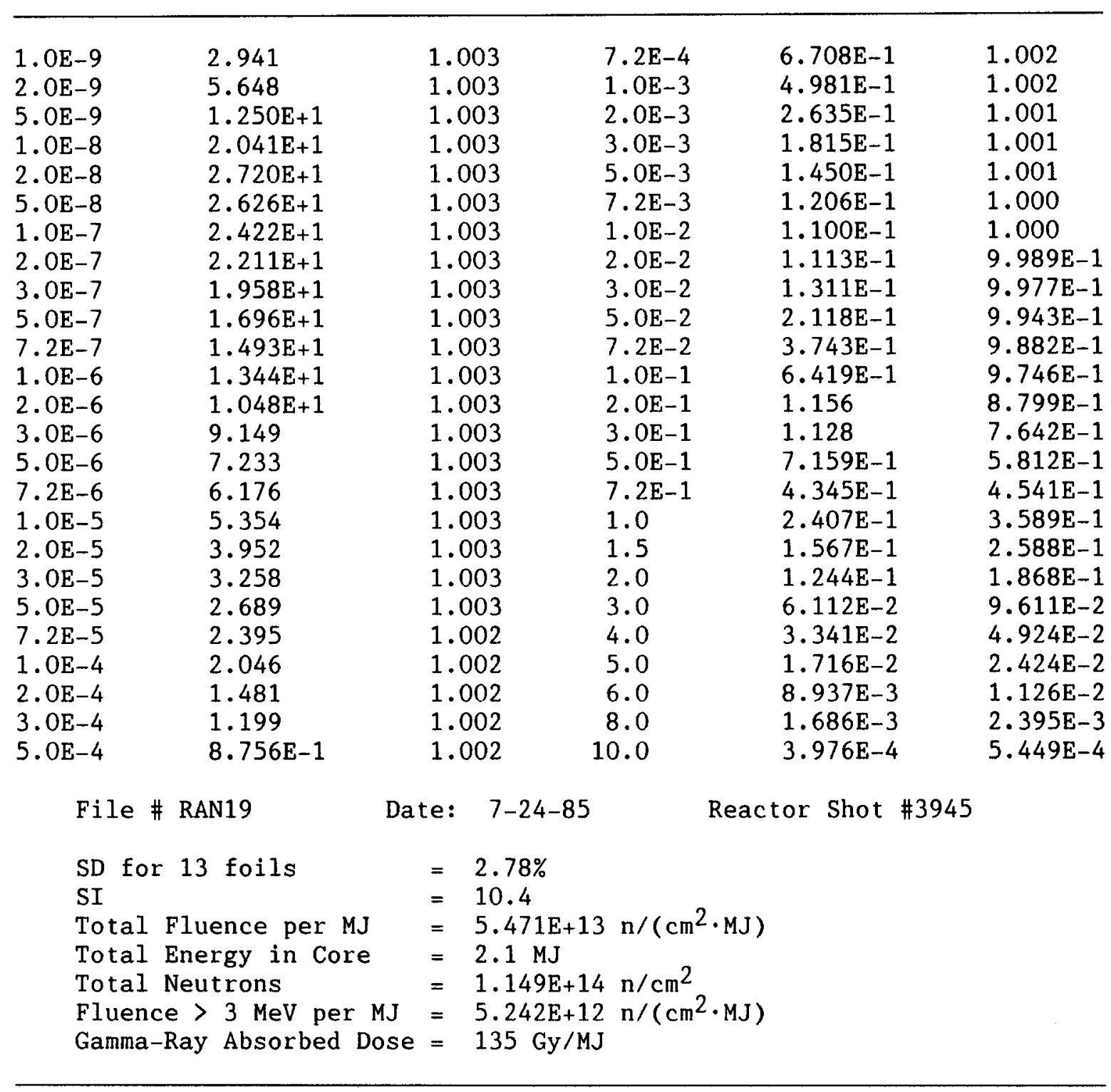




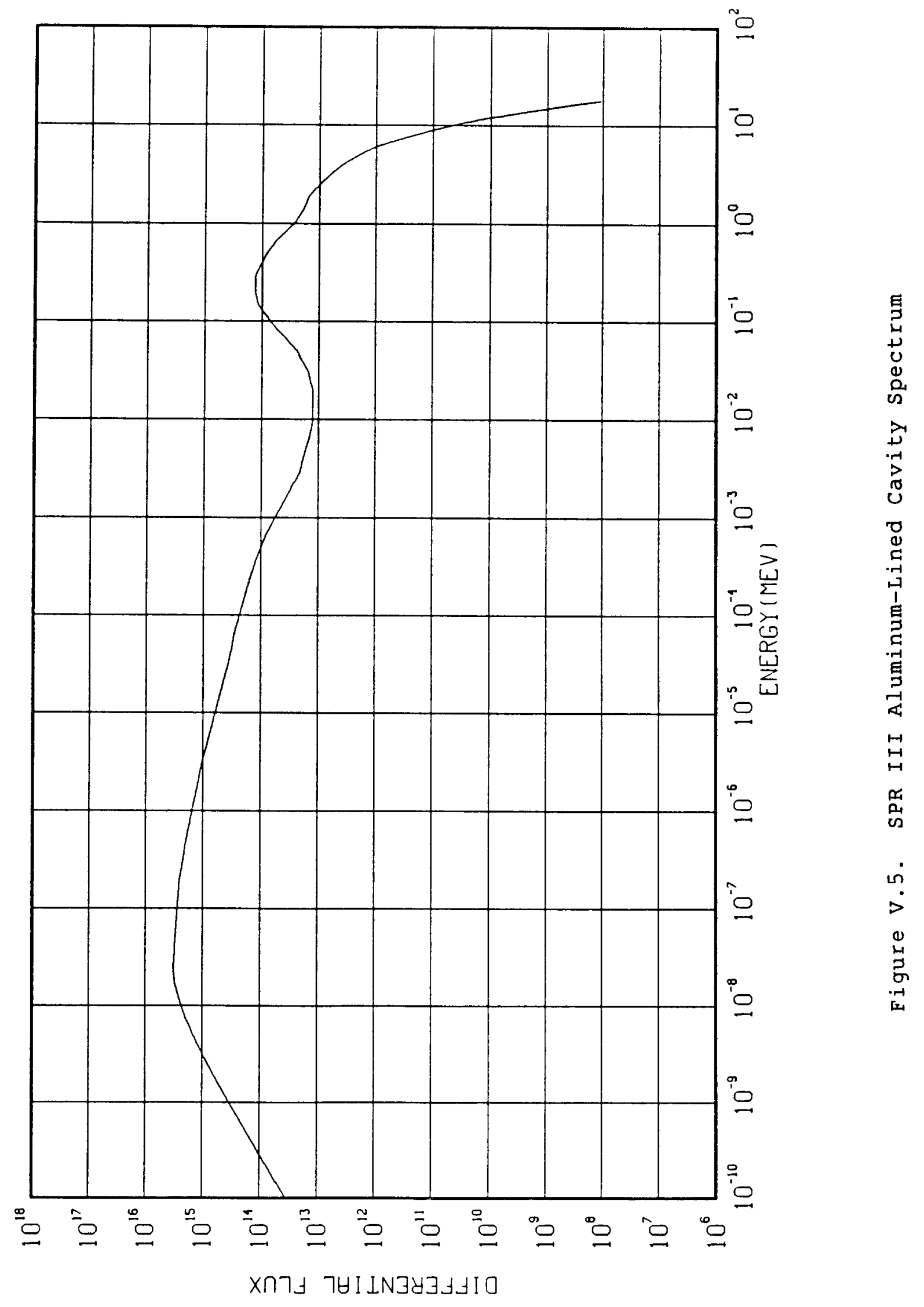




\section{(4) SPR III Leakage spectrum in Free Field at 17 Inches}

( SLEAK21)

With plenty of room available beside the reactor, the foils could be spaced far enough from the boron ball to eliminate the shadowing effect. Therefore, all of these exposures were made on the same reactor run. On a separate exposure, rows of nickel and sulfur foils were placed along the same azimuthal lines and at the same radii as the full foil set. No significant variation of induced activity with azimuth was found.

The steps taken during the adjustment process for this case were thoroughly discussed in chapter IV and are not repeated here. The set has turned out to be particularly compatible in that the resulting spectrum is very smooth, and the SD of the measured-to-calculated activities settled to only $0.81 \%$. As was the case with most of the other foil sets, the nickel inside the boron ball and the indium were deleted.

Table V.7 lists the foil activities, Table V.8 lists the spectrum values, and Figure V.6 shows a plot of the differential fluence versus energy. 
Table V.7

Foil Activities for SPR III 17-Inch

Free-Field Leakage spectrum

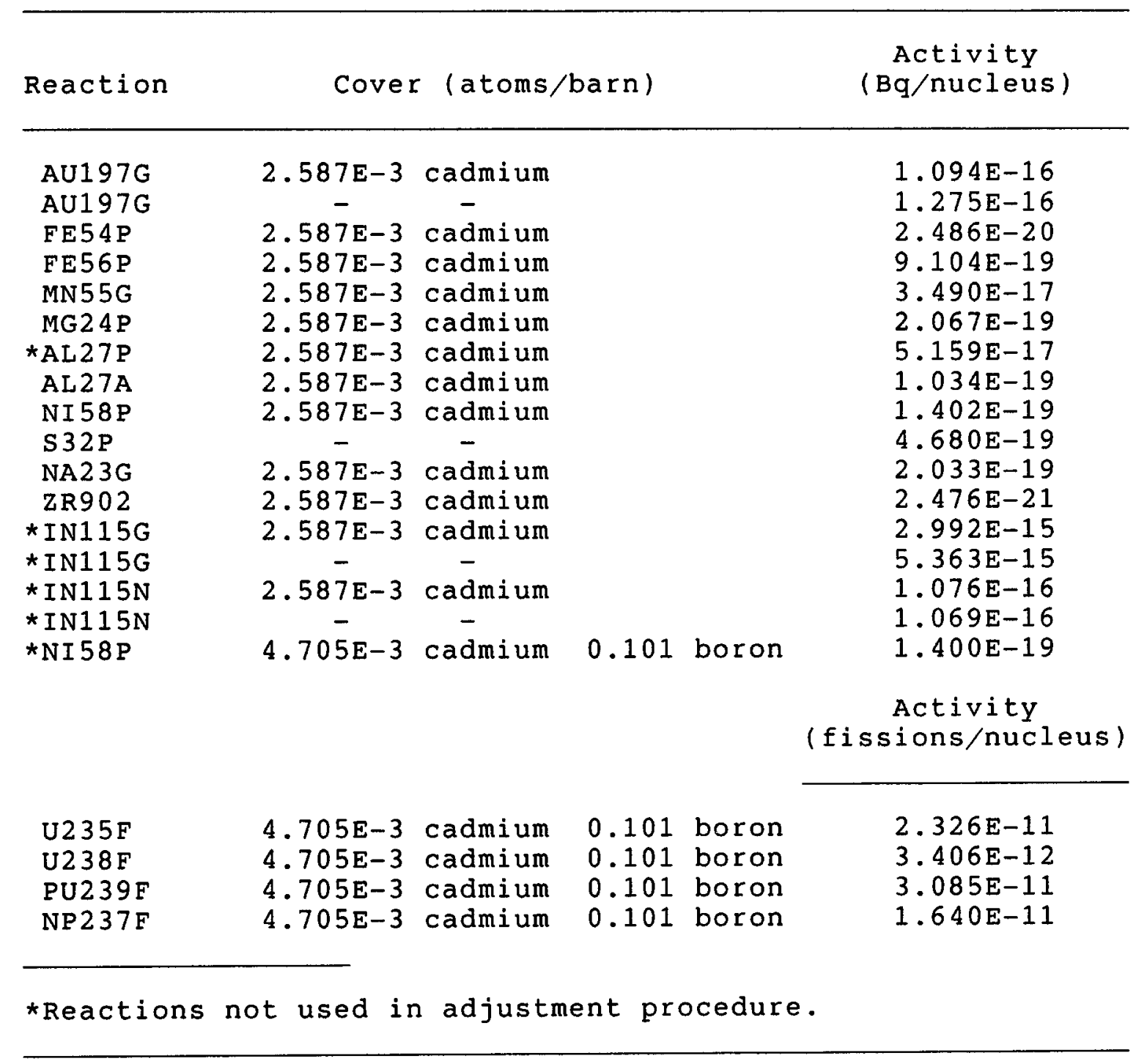


Table V. 8

SPR III 17-Inch Free-Field Leakage spectrum

$E(\mathrm{MeV})$ Differential Integral $E(\mathrm{MeV})$ Differential Integral

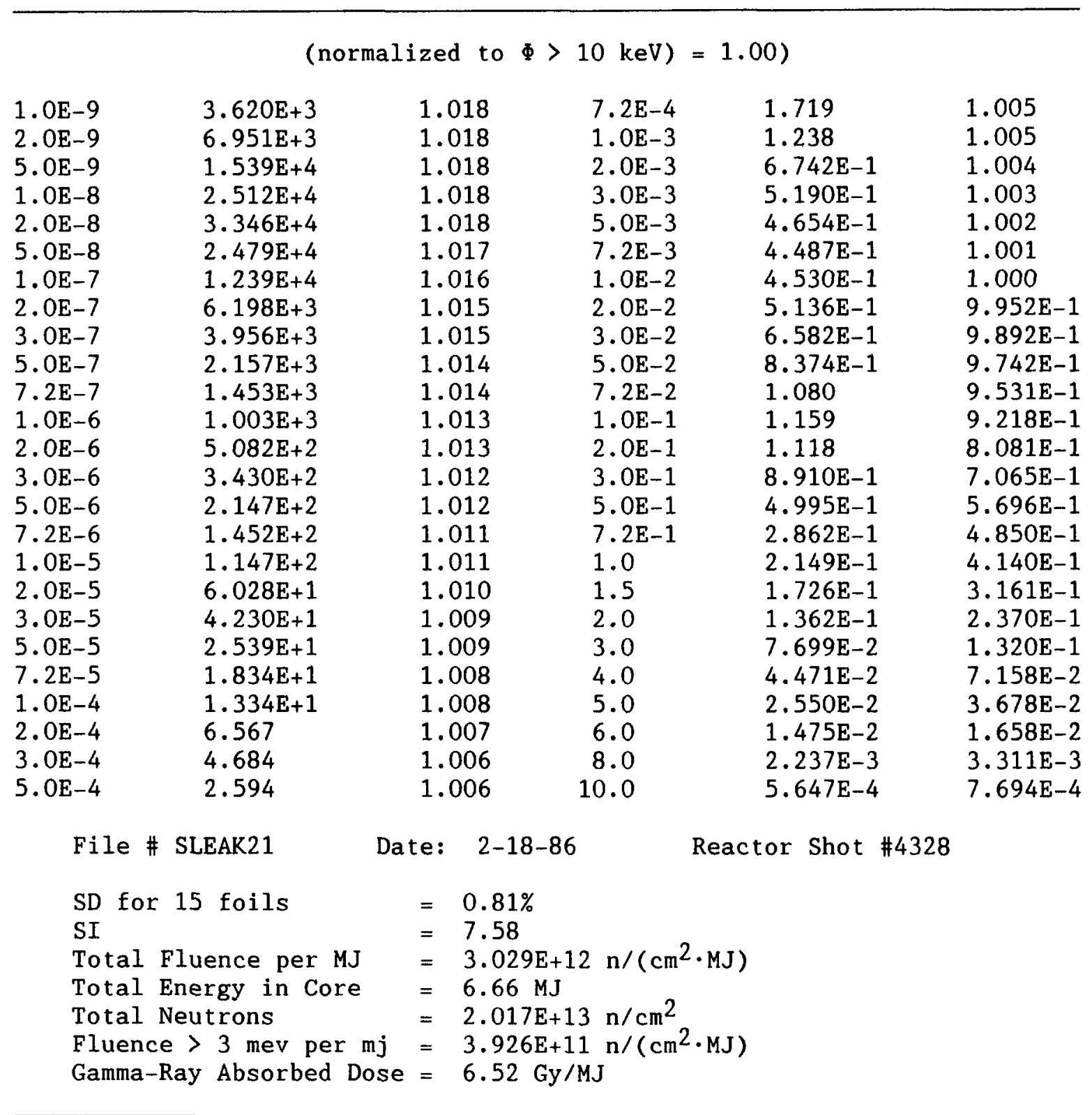




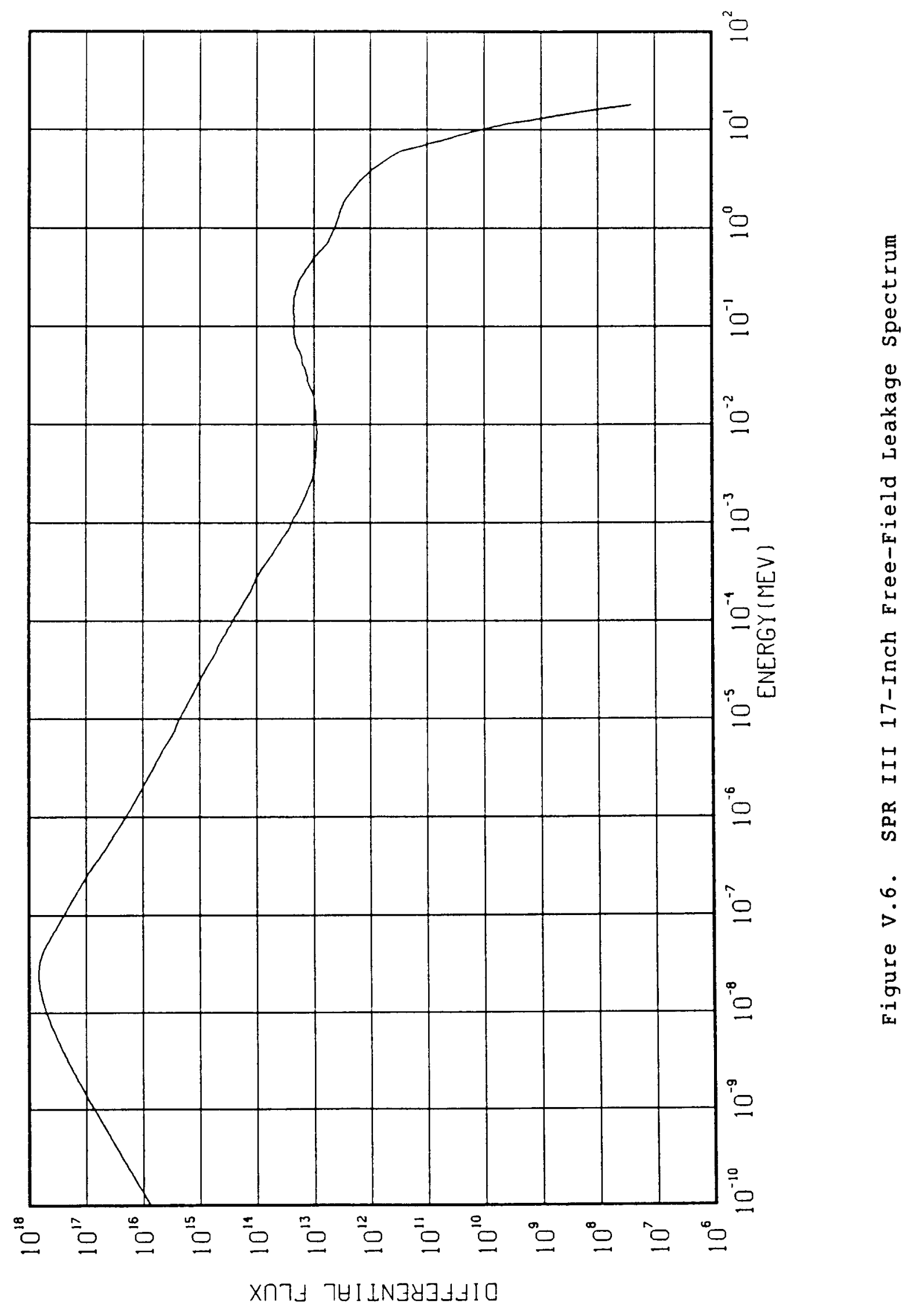



Layer Backed with Cadmium sheet (FLATCB24)

The measurement was conducted while another experimenter was in control of the total fluence for the run, so that some of the foils were inadequately exposed. Also, for some foils only one reading could be made before the activity dropped to a level that could not be read. The reactor was operated in pulse mode. The polyethylene was loaded with $20 \%$ by mass of cadmium oxide. The configuration is illustrated in Figure V.7.

The reaction $\mathrm{Mg} 24(\mathrm{n}, \mathrm{p}) \mathrm{Na} 24$ was excited so weakly that not enough counts were recorded to yield a reliable measurement. The reactions Fe54(n,p)Mn54, Al27(n,p)Mg27, and $N$ p237(n,f)FP produced so little activity that the counting program rejected the reactions as unidentifiable. We attempted to determine activities for these reactions by hand, but the resultant activities were so far removed from consistent fits to the majority of the reactions that they were ultimately deleted from the set. Background subtraction error is probably the explanation.

The counts recorded in the analyzer peaks for the reactions $\mathrm{Fe} 56(\mathrm{n}, \mathrm{p}) \mathrm{Mn} 56$, U235(n,f)FP, Pu239(n,f)FP, and U238(n,f)FP were used in hand calculations to yield activities that appeared to be consistent with the rest of the foil set, i.e., a compatible spectrum could be found. As for other data sets, the indium reactions were also deleted. The SANDII adjustment procedure was carried out with only the 11 foils that are not marked with asterisks in Table V.9.

The activity of the sodium foil at first seemed far too low to be reasonable. However, sodium exhibits a high response to thermal neutrons, which in this case were strongly attenuated by the cadmium layer on the rear surface of the front cadmium-polyethylene wall. When the low end of the trial spectrum was reduced (below the cadmium cutoff energy), the Na23(n,r)Na24 reaction became compatible with the rest of the foil set.

Although the data are of poor quality, and activities were (in a number of cases) determined by only a few hundred counts, the resulting spectrum does exhibit characteristics that appear to be correct. The SI is a low 6.4, as expected for a polyethylene shield arrangement that hardens the spectrum. In addition, a huge low-energy tail is generated by the downward scattering of neutrons in the polyethylene. Then the tail is cut off below thermal energies by the cadmium. Table V.10 lists the spectrum characteristics, and Figure V.8 shows the spectral shape obtained with 11 reactions. 


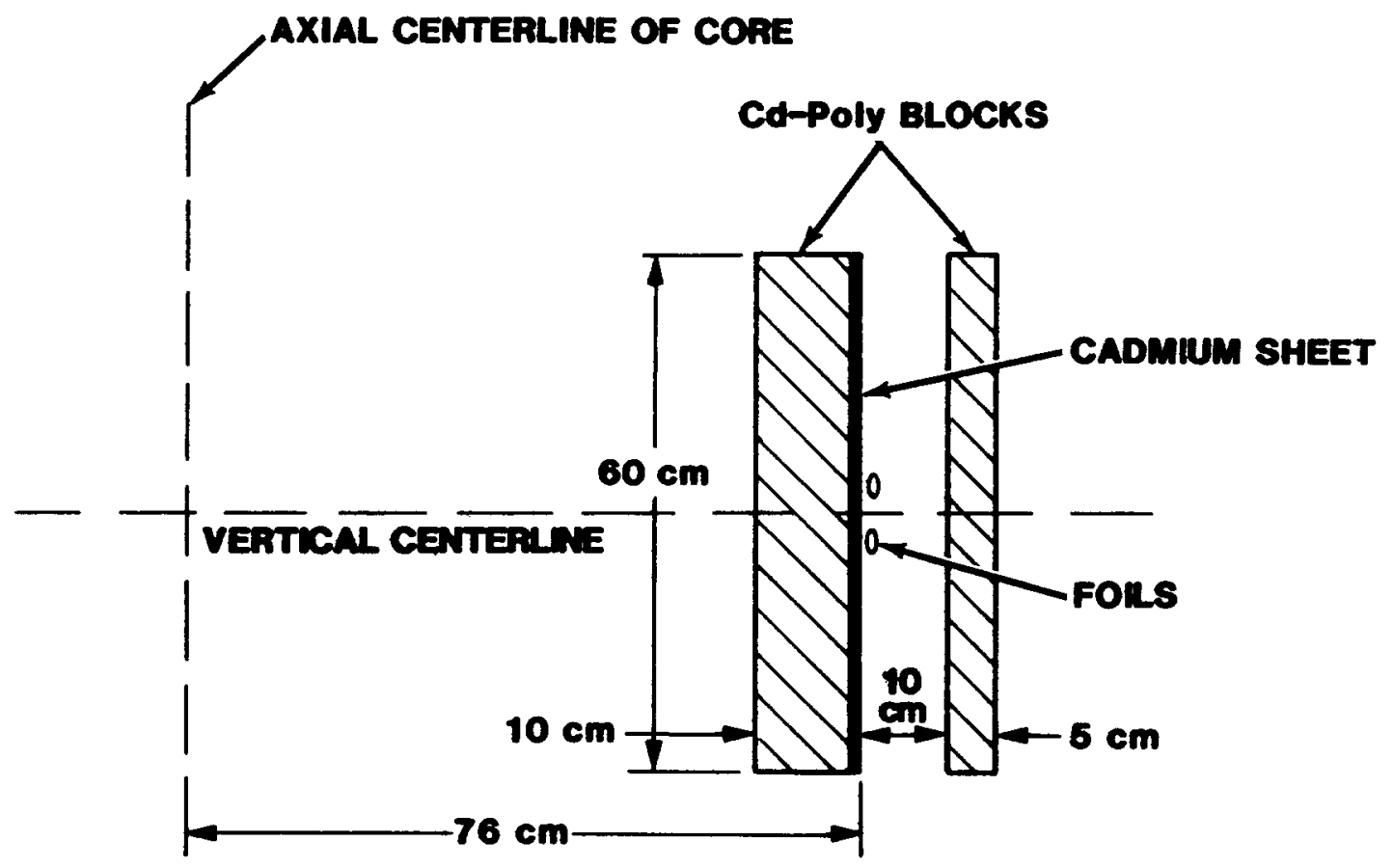

Figure V.7. Configuration of Foil set and Shielding Beside SPR III 
Table V.9

Foil Activities for SPR III Leakage spectrum Between Cadmium-Polyethylene Blocks

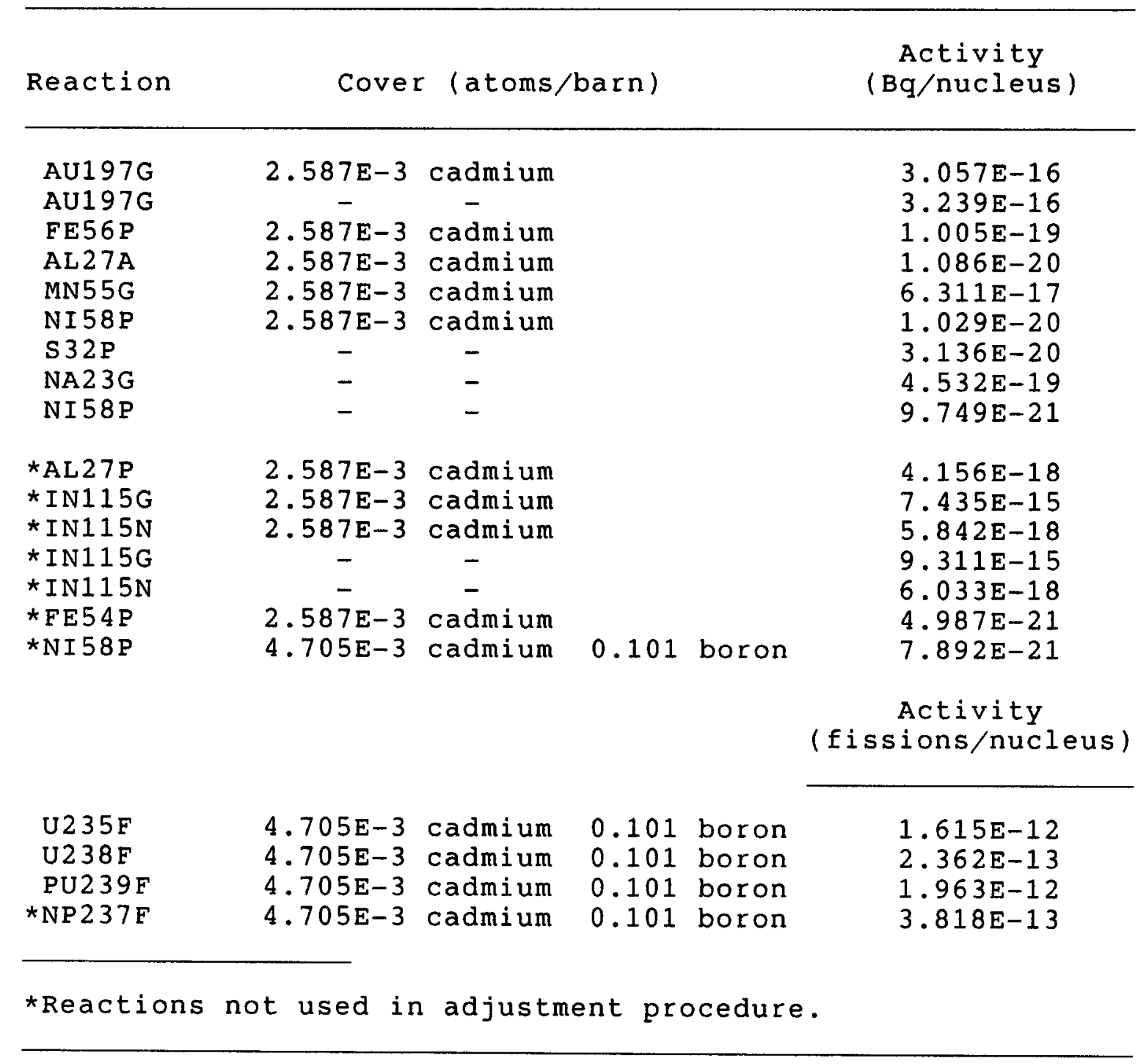


Table V.10

SPR III Leakage Spectrum Between

Cadmium-Polyethylene Blocks

\begin{tabular}{llllll} 
E (MeV) & Differential & Integral & $\mathrm{E}(\mathrm{MeV})$ & Differential & Integral \\
\hline & & & & & \\
$1.0 \mathrm{E}-9$ & $9.566 \mathrm{E}+2$ & 1.587 & $7.2 \mathrm{E}-4$ & $5.828 \mathrm{E}+1$ & 1.140 \\
$2.0 \mathrm{E}-9$ & $1.913 \mathrm{E}+3$ & 1.587 & $1.0 \mathrm{E}-3$ & $4.564 \mathrm{E}+1$ & 1.125 \\
$5.0 \mathrm{E}-9$ & $4.783 \mathrm{E}+3$ & 1.587 & $2.0 \mathrm{E}-3$ & $2.492 \mathrm{E}+1$ & 1.092 \\
$1.0 \mathrm{E}-8$ & $9.566 \mathrm{E}+3$ & 1.587 & $3.0 \mathrm{E}-3$ & $1.744 \mathrm{E}+1$ & 1.070 \\
$2.0 \mathrm{E}-8$ & $1.913 \mathrm{E}+4$ & 1.587 & $5.0 \mathrm{E}-3$ & $1.125 \mathrm{E}+1$ & 1.042 \\
$5.0 \mathrm{E}-8$ & $4.783 \mathrm{E}+4$ & 1.586 & $7.2 \mathrm{E}-3$ & 8.281 & 1.021 \\
$1.0 \mathrm{E}-7$ & $9.523 \mathrm{E}+4$ & 1.582 & $1.0 \mathrm{E}-2$ & 6.318 & 1.000 \\
$2.0 \mathrm{E}-7$ & $1.653 \mathrm{E}+5$ & 1.570 & $2.0 \mathrm{E}-2$ & 3.468 & $9.531 \mathrm{E}-1$ \\
$3.0 \mathrm{E}-7$ & $1.718 \mathrm{E}+5$ & 1.552 & $3.0 \mathrm{E}-2$ & 2.442 & $9.236 \mathrm{E}-1$ \\
$5.0 \mathrm{E}-7$ & $1.265 \mathrm{E}+5$ & 1.523 & $5.0 \mathrm{E}-2$ & 1.585 & $8.837 \mathrm{E}-1$ \\
$7.2 \mathrm{E}-7$ & $8.601 \mathrm{E}+4$ & 1.499 & $7.2 \mathrm{E}-2$ & 1.154 & $8.536 \mathrm{E}-1$ \\
$1.0 \mathrm{E}-6$ & $6.310 \mathrm{E}+4$ & 1.478 & $1.0 \mathrm{E}-1$ & $9.237 \mathrm{E}-1$ & $8.243 \mathrm{E}-1$ \\
$2.0 \mathrm{E}-6$ & $3.138 \mathrm{E}+4$ & 1.434 & $2.0 \mathrm{E}-1$ & $5.971 \mathrm{E}-1$ & $7.502 \mathrm{E}-1$ \\
$3.0 \mathrm{E}-6$ & $1.961 \mathrm{E}+4$ & 1.408 & $3.0 \mathrm{E}-1$ & $4.711 \mathrm{E}-1$ & $6.965 \mathrm{E}-1$ \\
$5.0 \mathrm{E}-6$ & $1.114 \mathrm{E}+4$ & 1.378 & $5.0 \mathrm{E}-1$ & $3.501 \mathrm{E}-1$ & $6.147 \mathrm{E}-1$ \\
$7.2 \mathrm{E}-6$ & $7.557 \mathrm{E}+3$ & 1.357 & $7.2 \mathrm{E}-1$ & $2.838 \mathrm{E}-1$ & $5.446 \mathrm{E}-1$ \\
$1.0 \mathrm{E}-5$ & $5.255 \mathrm{E}+3$ & 1.339 & 1.0 & $2.363 \mathrm{E}-1$ & $4.712 \mathrm{E}-1$ \\
$2.0 \mathrm{E}-5$ & $2.524 \mathrm{E}+3$ & 1.303 & 1.5 & $2.007 \mathrm{E}-1$ & $3.602 \mathrm{E}-1$ \\
$3.0 \mathrm{E}-5$ & $1.617 \mathrm{E}+3$ & 1.282 & 2.0 & $1.518 \mathrm{E}-1$ & $2.700 \mathrm{E}-1$ \\
$5.0 \mathrm{E}-5$ & $9.433 \mathrm{E}+2$ & 1.257 & 3.0 & $8.097 \mathrm{E}-2$ & $1.558 \mathrm{E}-1$ \\
$7.2 \mathrm{E}-5$ & $6.248 \mathrm{E}+2$ & 1.240 & 4.0 & $4.511 \mathrm{E}-2$ & $9.352 \mathrm{E}-2$ \\
$1.0 \mathrm{E}-4$ & $4.477 \mathrm{E}+2$ & 1.224 & 5.0 & $2.942 \mathrm{E}-2$ & $5.663 \mathrm{E}-2$ \\
$2.0 \mathrm{E}-4$ & $2.072 \mathrm{E}+2$ & 1.194 & 6.0 & $2.236 \mathrm{E}-2$ & $3.042 \mathrm{E}-2$ \\
$3.0 \mathrm{E}-4$ & $1.339 \mathrm{E}+2$ & 1.177 & 8.0 & $5.445 \mathrm{E}-3$ & $5.965 \mathrm{E}-3$ \\
$5.0 \mathrm{E}-4$ & $8.228 \mathrm{E}+1$ & 1.155 & 10.0 & $7.783 \mathrm{E}-4$ & $1.105 \mathrm{E}-3$
\end{tabular}

File \# FLATCB24 Date: 11-14-84 Reactor Shot \#3441

$\begin{array}{ll}\text { SD for } 11 \text { foils } & =2.76 \% \\ \text { SI } & =6.42 \\ \text { Total Fluence per MJ } & =2.953 \mathrm{E}+11 \mathrm{n} /\left(\mathrm{cm}^{2} \cdot \mathrm{MJ}\right) \\ \text { Total Energy in Core } & =6.33 \mathrm{MJ} \\ \text { Total Neutrons } & =1.869 \mathrm{E}+12 \mathrm{n} / \mathrm{cm}^{2} \\ \text { Fluence }>3 \mathrm{MeV} \text { per MJ } & =2.897 \mathrm{E}+10 \mathrm{n} /\left(\mathrm{cm}^{2} \cdot \mathrm{MJ}\right) \\ \text { Gamma-Ray Absorbed Dose } & =14.3 \mathrm{~Gy} / \mathrm{MJ}\end{array}$




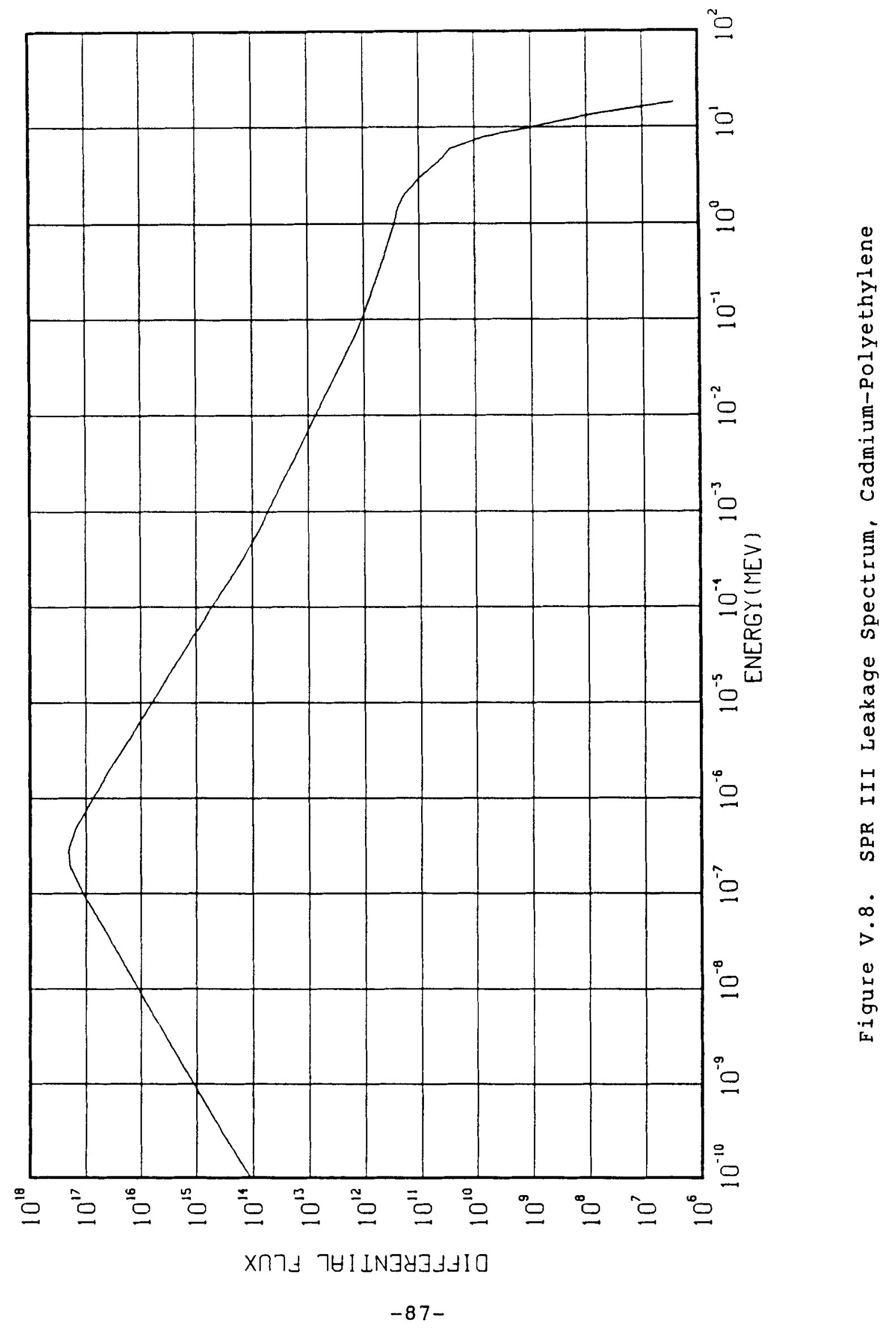


(6) Leakage spectrum Between second set of CadmiumPolyethylene and Polyethylene Blocks (CDPOC7)

This geometry was tested by request from an experimenter who wanted to know the neutron spectrum in a configuration designed to produce a high gamma-ray radiation field. This configuration, like the SPR III 17-inch leakage configuration, was one of those chosen as a benchmark field for calculations of the neutron spectrum with the MORSE coupled $n, r$ Monte carlo code.[24] The agreement between the calculated and measured spectra was reasonably good above $10 \mathrm{keV}$ if account is taken of the fact that the measured activities were obtained $5 \mathrm{~cm}$ closer to the core than those of the calculation.

The configuration is shown in Figure V.9. There is much less cadmium-loaded polyethylene in this setup than in the previous case considered, and no cadmium sheet is mounted on the back of the front wall as was the case in the previous example. Again, the exposures were quite low, so that the measurements of the magnesium and zirconium activities were missed entirely. The foils of sodium and manganese were read only once. Table $V .11$ lists the reactions measured. only those without asterisks were used in the adjustment procedure. The low number of detector counts, together with the single readings on some of the foils, caused a larger-than-usual statistical error in the activities ( 3 to $10 \%$ ). In this case, no activities were calculated by hand, as was done in the previous case. Note also that the cadmium-covered indium foil (IN115N) showed more activity than the bare indium foil, providing credence to the conjecture that this reaction is excited by gamma rays.

Figure V.10 shows a comparison of the spectra obtained from two independent trials. The first was obtained by initiating the adjustment process with the flat trial and working to a solution (before any calculated trial became available) by the techniques described in chapter IV. The second spectrum is the result of using as a trial a smoothed spectrum from a MORSE Monte Carlo calculation. One can see that the differences are small. We believe that the structure seen in the spectra above $1 \mathrm{MeV}$ is due to inaccuracies in the measured activities. We still pushed the code to iterate until the SD was less than $3 \%$. It took 13 iterations to lower the SD from 4 to $3 \%$ Table V.12 lists the spectrum values, and Figure V.11 shows the spectrum that resulted from a smoother trial and the deletion of the $\operatorname{In} 115\left(n, n^{\prime}\right)$ In 115 reaction. This final spectrum is slightly different from those in Figure v.10 because a small correction to the fission-foil activities was applied after the comparisons were made. 
This configuration is very similar to the previous example--leakage spectra between cadmium-loaded polyethylene blocks. Yet the SI is quite different (6.4 in Case 5 , versus 8.0 here). In order to demonstrate that the difference is caused by a difference in activity set rather than a difference in trial function, the trial of case 6 was substituted into the input file of Case 5. The SI obtained was identical to the 6.4 of Case 5, proving that the activity set, not the trial, determined the spectrum shape.

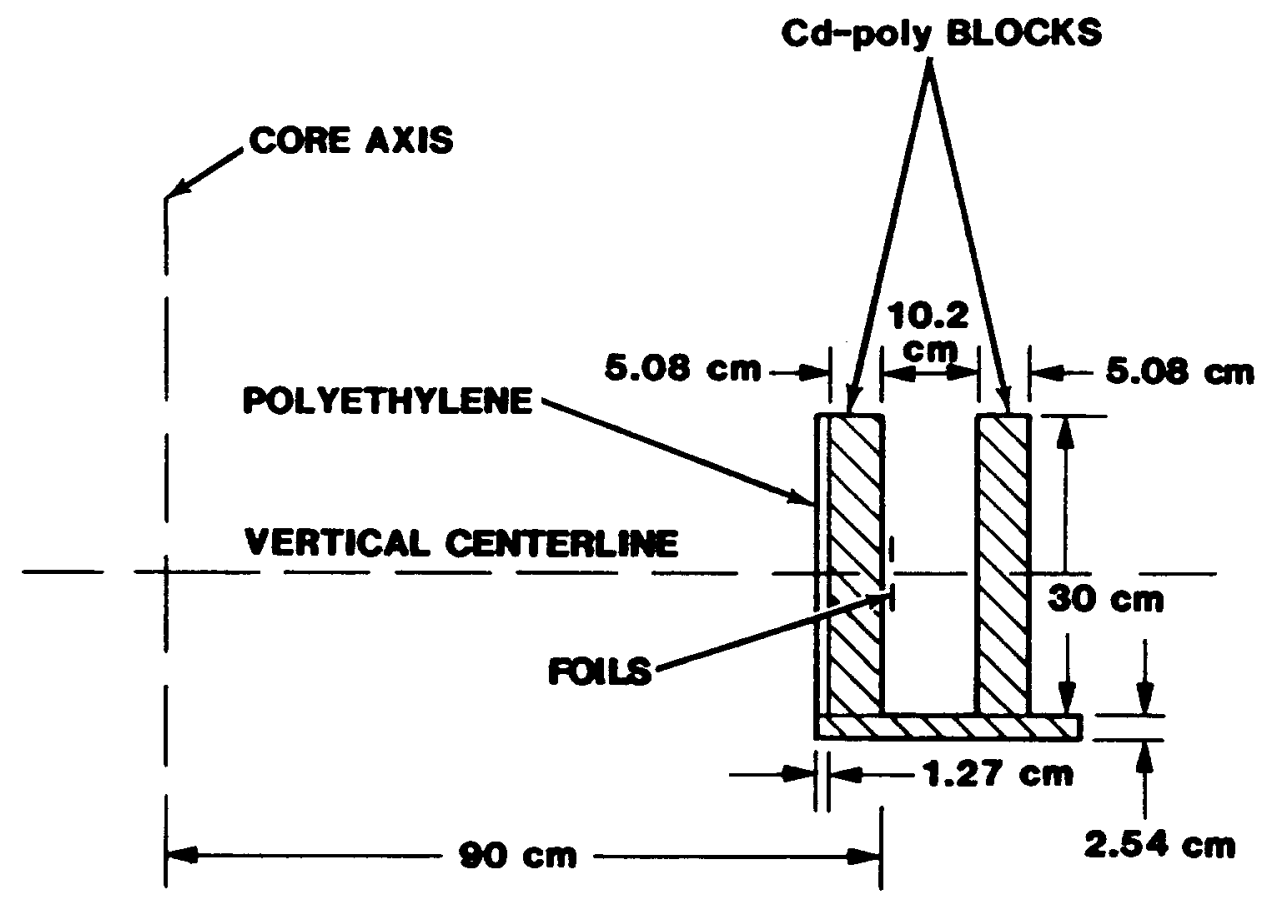

The blocks were $60 \mathrm{~cm}$ long in the horizontal directlon

Figure V.9. Configuration for second Cadmium-Polyethylene and Polyethylene wall structure 
Table V.11

Foil Activities for SPR III Leakage spectra Between Cadmium-Polyethylene and Polyethylene Blocks

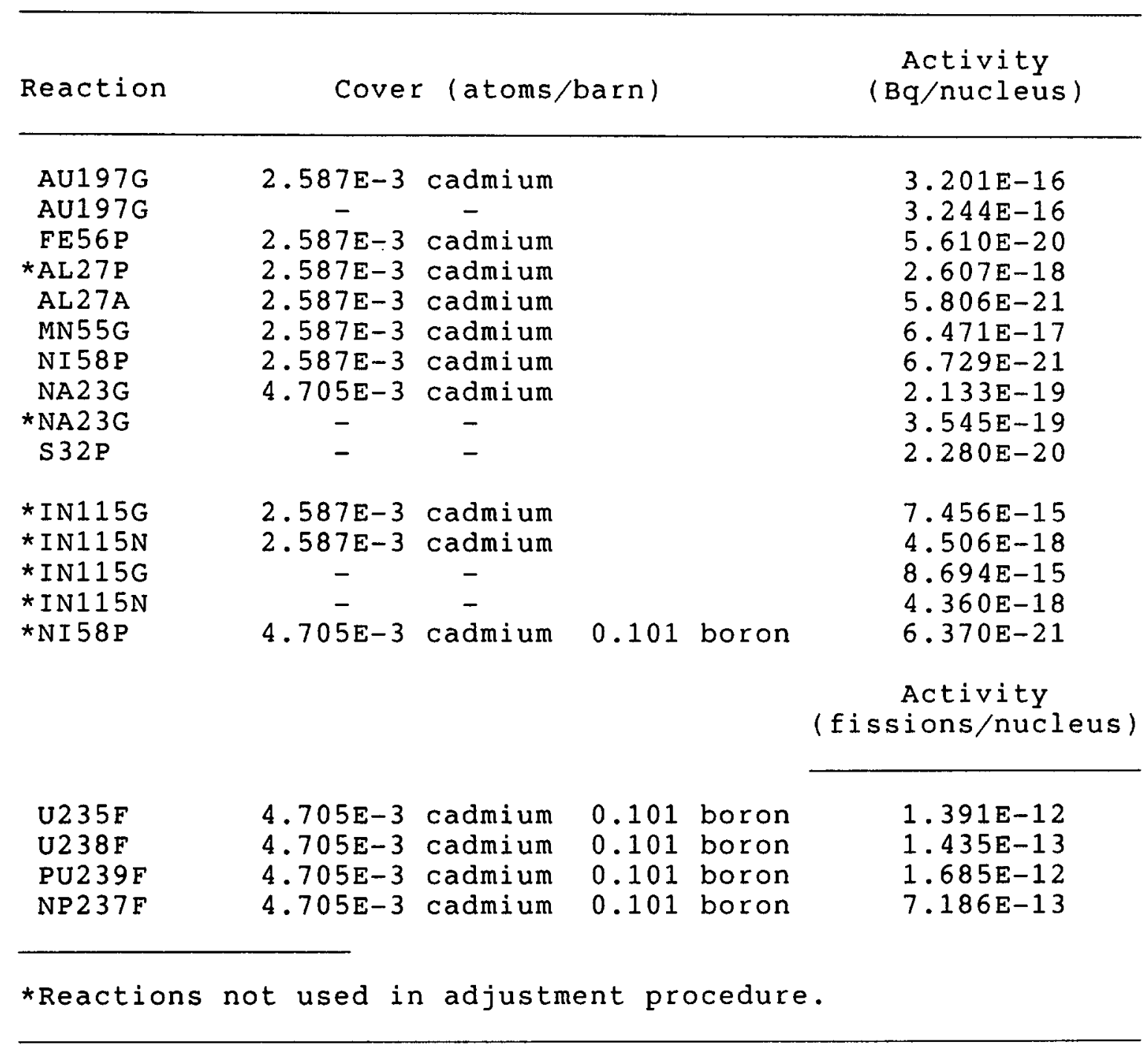




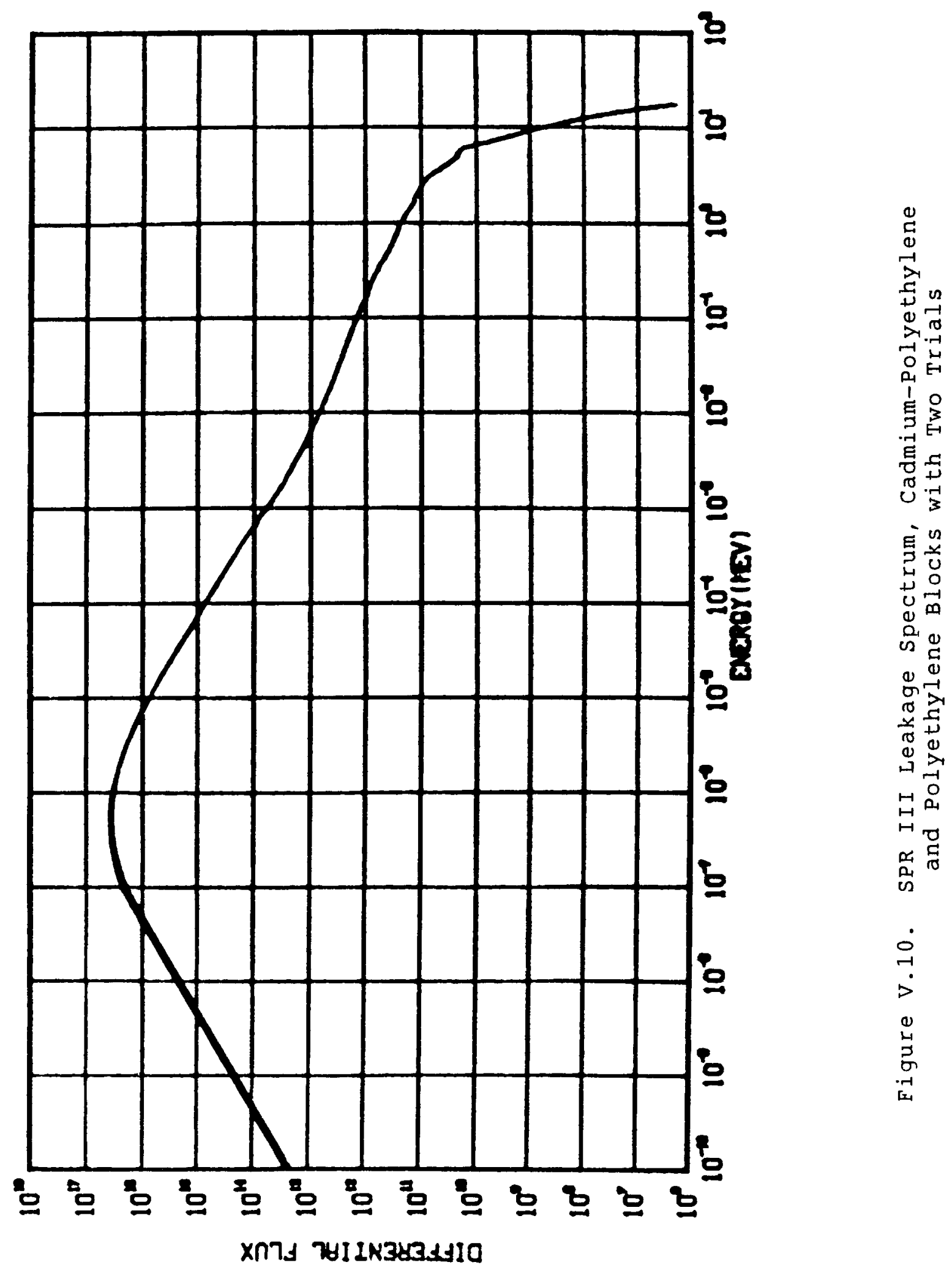


Table V.12

SPR III Leakage Spectrum Between

Cadmium-Polyethylene and Polyethylene walls

\begin{tabular}{llllll}
\hline E (MeV) & Differential & Integral & $\mathrm{E}(\mathrm{MeV})$ & Differential & Integral \\
\hline & & & & & \\
$1.0 \mathrm{E}-9$ & $2.423 \mathrm{E}+2$ & 1.656 & $7.2 \mathrm{E}-4$ & $8.837 \mathrm{E}+1$ & 1.162 \\
$2.0 \mathrm{E}-9$ & $4.846 \mathrm{E}+2$ & 1.656 & $1.0 \mathrm{E}-3$ & $5.720 \mathrm{E}+1$ & 1.141 \\
$5.0 \mathrm{E}-9$ & $1.211 \mathrm{E}+3$ & 1.656 & $2.0 \mathrm{E}-3$ & $2.763 \mathrm{E}+1$ & 1.101 \\
$1.0 \mathrm{E}-8$ & $2.422 \mathrm{E}+3$ & 1.656 & $3.0 \mathrm{E}-3$ & $2.036 \mathrm{E}+1$ & 1.077 \\
$2.0 \mathrm{E}-8$ & $4.838 \mathrm{E}+3$ & 1.656 & $5.0 \mathrm{E}-3$ & $1.200 \mathrm{E}+1$ & 1.045 \\
$5.0 \mathrm{E}-8$ & $1.208 \mathrm{E}+4$ & 1.656 & $7.2 \mathrm{E}-3$ & 8.997 & 1.022 \\
$1.0 \mathrm{E}-7$ & $2.390 \mathrm{E}+4$ & 1.655 & $1.0 \mathrm{E}-2$ & 6.785 & 1.000 \\
$2.0 \mathrm{E}-7$ & $3.248 \mathrm{E}+4$ & 1.652 & $2.0 \mathrm{E}-2$ & 4.025 & $9.477 \mathrm{E}-1$ \\
$3.0 \mathrm{E}-7$ & $3.612 \mathrm{E}+4$ & 1.649 & $3.0 \mathrm{E}-2$ & 3.077 & $9.124 \mathrm{E}-1$ \\
$5.0 \mathrm{E}-7$ & $3.872 \mathrm{E}+4$ & 1.641 & $5.0 \mathrm{E}-2$ & 2.164 & $8.605 \mathrm{E}-1$ \\
$7.2 \mathrm{E}-7$ & $3.746 \mathrm{E}+4$ & 1.633 & $7.2 \mathrm{E}-2$ & 1.612 & $8.190 \mathrm{E}-1$ \\
$1.0 \mathrm{E}-6$ & $3.435 \mathrm{E}+4$ & 1.623 & $1.0 \mathrm{E}-1$ & 1.334 & $7.774 \mathrm{E}-1$ \\
$2.0 \mathrm{E}-6$ & $2.542 \mathrm{E}+4$ & 1.593 & $2.0 \mathrm{E}-1$ & $8.102 \mathrm{E}-1$ & $6.734 \mathrm{E}-1$ \\
$3.0 \mathrm{E}-6$ & $2.018 \mathrm{E}+4$ & 1.570 & $3.0 \mathrm{E}-1$ & $6.170 \mathrm{E}-1$ & $6.014 \mathrm{E}-1$ \\
$5.0 \mathrm{E}-6$ & $1.386 \mathrm{E}+4$ & 1.536 & $5.0 \mathrm{E}-1$ & $3.904 \mathrm{E}-1$ & $5.017 \mathrm{E}-1$ \\
$7.2 \mathrm{E}-6$ & $9.906 \mathrm{E}+3$ & 1.510 & $7.2 \mathrm{E}-1$ & $2.855 \mathrm{E}-1$ & $4.269 \mathrm{E}-1$ \\
$1.0 \mathrm{E}-5$ & $7.452 \mathrm{E}+3$ & 1.486 & 1.0 & $2.047 \mathrm{E}-1$ & $3.569 \mathrm{E}-1$ \\
$2.0 \mathrm{E}-5$ & $3.935 \mathrm{E}+3$ & 1.431 & 1.5 & $1.332 \mathrm{E}-1$ & $2.710 \mathrm{E}-1$ \\
$3.0 \mathrm{E}-5$ & $2.586 \mathrm{E}+3$ & 1.399 & 2.0 & $1.046 \mathrm{E}-1$ & $2.109 \mathrm{E}-1$ \\
$5.0 \mathrm{E}-5$ & $1.467 \mathrm{E}+3$ & 1.359 & 3.0 & $6.609 \mathrm{E}-2$ & $1.257 \mathrm{E}-1$ \\
$7.2 \mathrm{E}-5$ & $1.019 \mathrm{E}+3$ & 1.332 & 4.0 & $3.938 \mathrm{E}-2$ & $7.336 \mathrm{E}-2$ \\
$1.0 \mathrm{E}-4$ & $7.509 \mathrm{E}+2$ & 1.307 & 5.0 & $2.547 \mathrm{E}-2$ & $4.101 \mathrm{E}-2$ \\
$2.0 \mathrm{E}-4$ & $3.712 \mathrm{E}+2$ & 1.253 & 6.0 & $1.677 \mathrm{E}-2$ & $1.953 \mathrm{E}-2$ \\
$3.0 \mathrm{E}-4$ & $2.307 \mathrm{E}+2$ & 1.223 & 8.0 & $2.754 \mathrm{E}-3$ & $3.874 \mathrm{E}-3$ \\
$5.0 \mathrm{E}-4$ & $1.394 \mathrm{E}+2$ & 1.187 & $1.0 \mathrm{E}+1$ & $6.325 \mathrm{E}-4$ & $8.996 \mathrm{E}-4$ \\
& & & & &
\end{tabular}

$\begin{array}{ll}\text { File \# CDPOC7 Date: } & 7-04-85 \quad \text { Reactor Shot \#3902 } \\ & =2.48 \% \\ \text { SD for } 12 \text { foils } & 7.96 \\ \text { SI } & 5.583 \mathrm{E}+11 \mathrm{n} /\left(\mathrm{cm}^{2} \cdot \mathrm{MJ}\right) \\ \text { Total Fluence per MJ } & 2.97 \mathrm{MJ} \\ \text { Total Energy in Core } & 1.658 \mathrm{E}+12 \mathrm{n} / \mathrm{cm}^{2} \\ \text { Total Neutrons } & 4.239 \mathrm{E}+10 \mathrm{n} /\left(\mathrm{cm}^{2} \cdot \mathrm{MJ}\right) \\ \text { Fluence }>3 \mathrm{MeV} \text { per MJ } & =6.77 \mathrm{~Gy} / \mathrm{MJ}\end{array}$




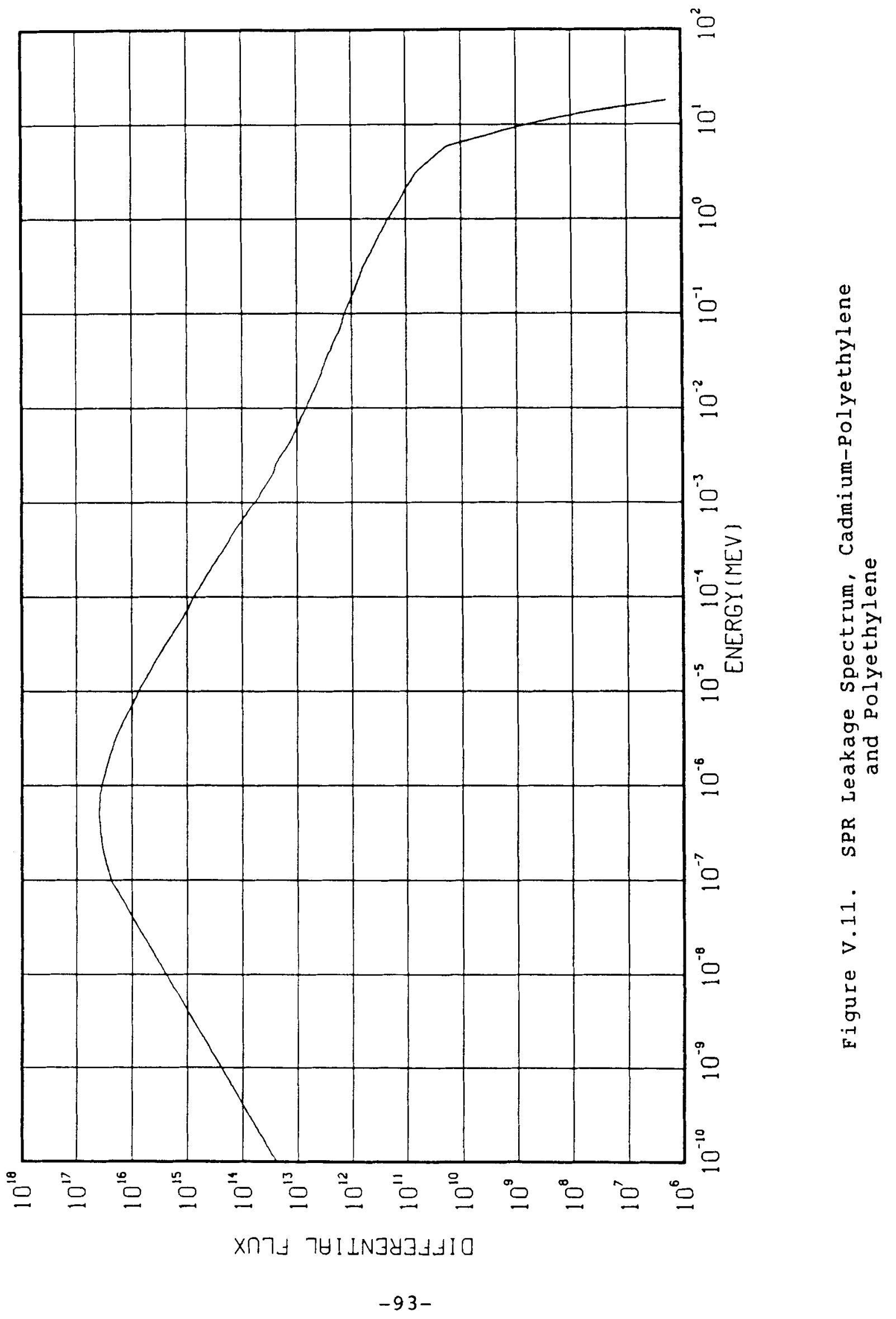



(BSHLD20)

One experimenter required knowledge of the spectra inside a shielded box that was designed to provide a collimated neutron and gamma-ray beam inside the reactor room (Kiva). The shielded box is shown in Figure V.12. The principal components were borated polyethylene to slow down and capture neutrons, a layer of boral (boron carbide in an aluminum plate) to block thermal neutrons, and a layer of lead to attenuate the remaining neutrons. The lead also attenuated the kiva gamma rays and the gamma rays generated by neutron capture in the boron.

For this foil set, the front collimator opening was plugged with boron-loaded polyethylene and lead, so that the spectrum inside the shield and away from the collimated beam could be measured. The foils were placed against the inside surface of the front wall, as shown in Figure V.12. (Radiation can return through the beam exit port from the kiva wall, but this return radiation was certainly less than that which would return from any inner wall that intercepted the beam just a few inches from the detectors.)

This shielded geometry was difficult to characterize because the fluence level was very low. The magnesium and Fe54 reaction activities were too weak to measure, and the activities of Fe56 and U238 were calculated by hand because only a few hundred counts were obtained. The aluminum, sulfur, and sodium foils were counted once. The exposed foils and their activities are listed in Table V.13. As before, the foils used in the spectrum adjustment do not have an asterisk beside them. The spectrum characteristics are listed in Table V.14, and the spectrum is shown in Figure V.13. The reader may notice that the nickel foil from the boron ball is included in this case. It was left in the analysis because there were so few other reactions. Its inclusion lowered the SI by $0.5 \%$.

The most interesting feature here is the very high SI of 18.8. This is characteristic of spectra whose high-energy neutrons are strongly attenuated by inelastic scattering in lead. 
Note: Dimenslons are in inches.

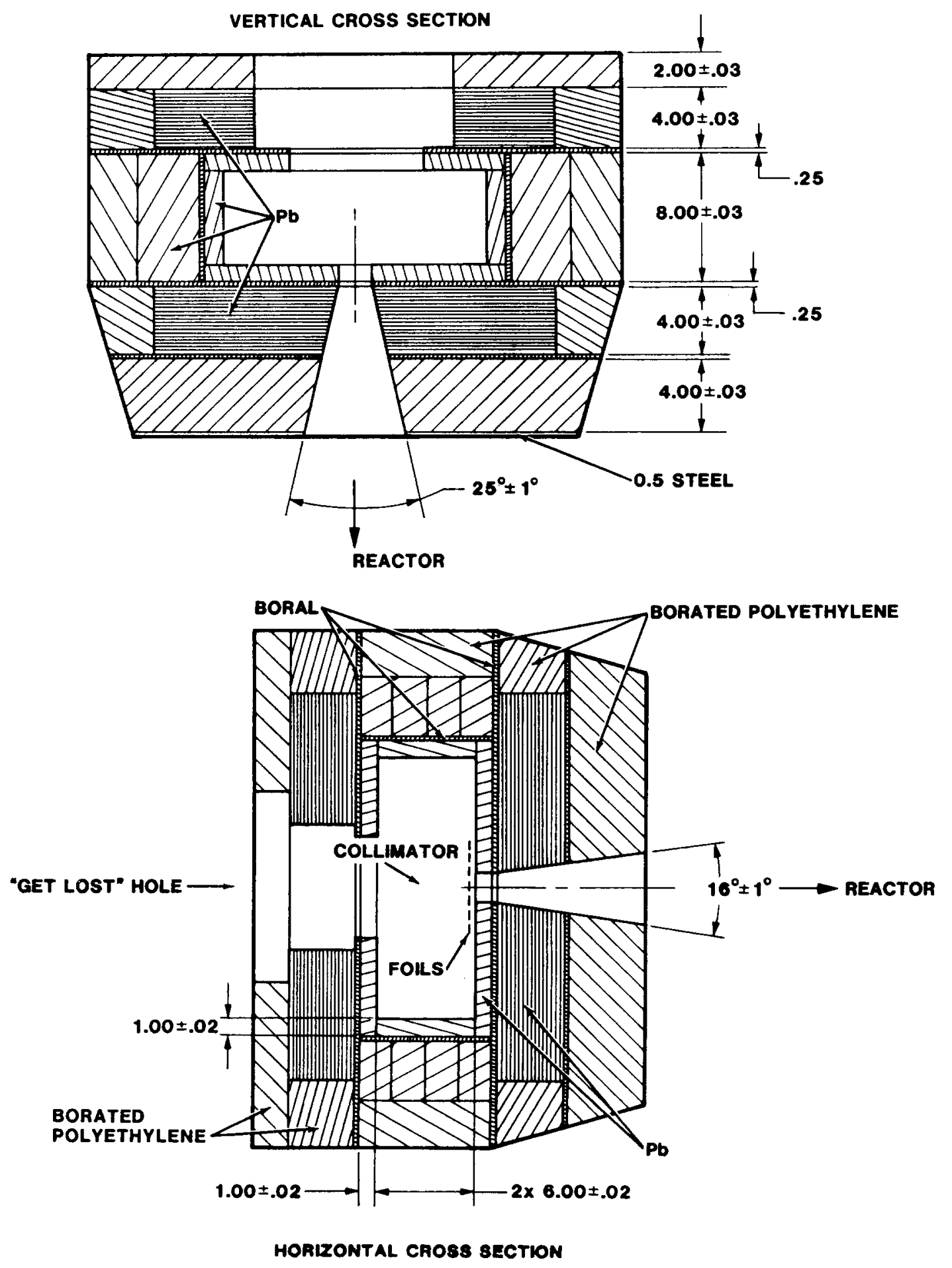

Figure V.12. Drawing of Beam Shield Collimator 
Table V.13

Foil Activities from Plugged Beam Shield Beside SPR III

\begin{tabular}{|c|c|c|c|c|}
\hline Reaction & \multicolumn{3}{|c|}{ Cover (atoms/barn) } & $\begin{array}{c}\text { Activity } \\
\text { (Bq/nucleus) }\end{array}$ \\
\hline $\begin{array}{l}\text { AU197G } \\
\text { AU197G } \\
\text { MN55G } \\
\text { *AL27P } \\
\text { AL27A } \\
\text { *FE56P } \\
\text { NI58P } \\
\text { S32P } \\
\text { NA23G }\end{array}$ & $\begin{array}{c}2.587 \mathrm{E}-3 \\
- \\
2.587 \mathrm{E}-3 \\
2.587 \mathrm{E}-3 \\
2.587 \mathrm{E}-3 \\
2.587 \mathrm{E}-3 \\
2.587 \mathrm{E}-3 \\
- \\
-\end{array}$ & $\begin{array}{l}\text { cadmi um } \\
- \\
\text { cadmium } \\
\text { cadmium } \\
\text { cadmium } \\
\text { cadmium } \\
\text { cadmium } \\
- \\
-\end{array}$ & & $\begin{array}{l}2.474 \mathrm{E}-17 \\
2.547 \mathrm{E}-17 \\
1.641 \mathrm{E}-17 \\
8.046 \mathrm{E}-19 \\
1.995 \mathrm{E}-21 \\
1.825 \mathrm{E}-20 \\
2.525 \mathrm{E}-21 \\
8.531 \mathrm{E}-21 \\
6.982 \mathrm{E}-20\end{array}$ \\
\hline $\begin{array}{l}\text { *IN115G } \\
\text { *IN115N } \\
\text { *IN115G } \\
\text { *IN115N } \\
\text { NI } 58 \mathrm{P}\end{array}$ & $\begin{array}{c}2.587 E-3 \\
2.587 E-3 \\
- \\
\overline{-} \\
4.705 E-3\end{array}$ & $\begin{array}{l}\text { cadmium } \\
\text { cadmium } \\
- \\
- \\
\text { cadmium }\end{array}$ & 0.101 boron & $\begin{array}{c}4.061 \mathrm{E}-16 \\
2.585 \mathrm{E}-18 \\
6.019 \mathrm{E}-16 \\
2.588 \mathrm{E}-18 \\
2.341 \mathrm{E}-21 \\
\\
\text { Activity } \\
\text { (fissions/nucleus) }\end{array}$ \\
\hline $\begin{array}{l}\text { U2 } 35 F \\
\text { U2 } 38 F \\
\text { PU239F } \\
\text { NP2 } 37 F\end{array}$ & $\begin{array}{l}4.705 E-3 \\
4.705 E-3 \\
4.705 E-3 \\
4.705 E-3\end{array}$ & $\begin{array}{l}\text { cadmium } \\
\text { cadmium } \\
\text { cadmium } \\
\text { cadmium }\end{array}$ & $\begin{array}{l}0.101 \text { boron } \\
0.101 \text { boron } \\
0.101 \text { boron } \\
0.101 \text { boron }\end{array}$ & $\begin{array}{l}1.064 \mathrm{E}-12 \\
8.039 \mathrm{E}-14 \\
1.236 \mathrm{E}-12 \\
5.309 \mathrm{E}-13\end{array}$ \\
\hline
\end{tabular}


Table V.14

SPR III Leakage Spectrum in Plugged Beam Shield

$E(M e V)$ Differential Integral $E(M e V)$ Differential Integral

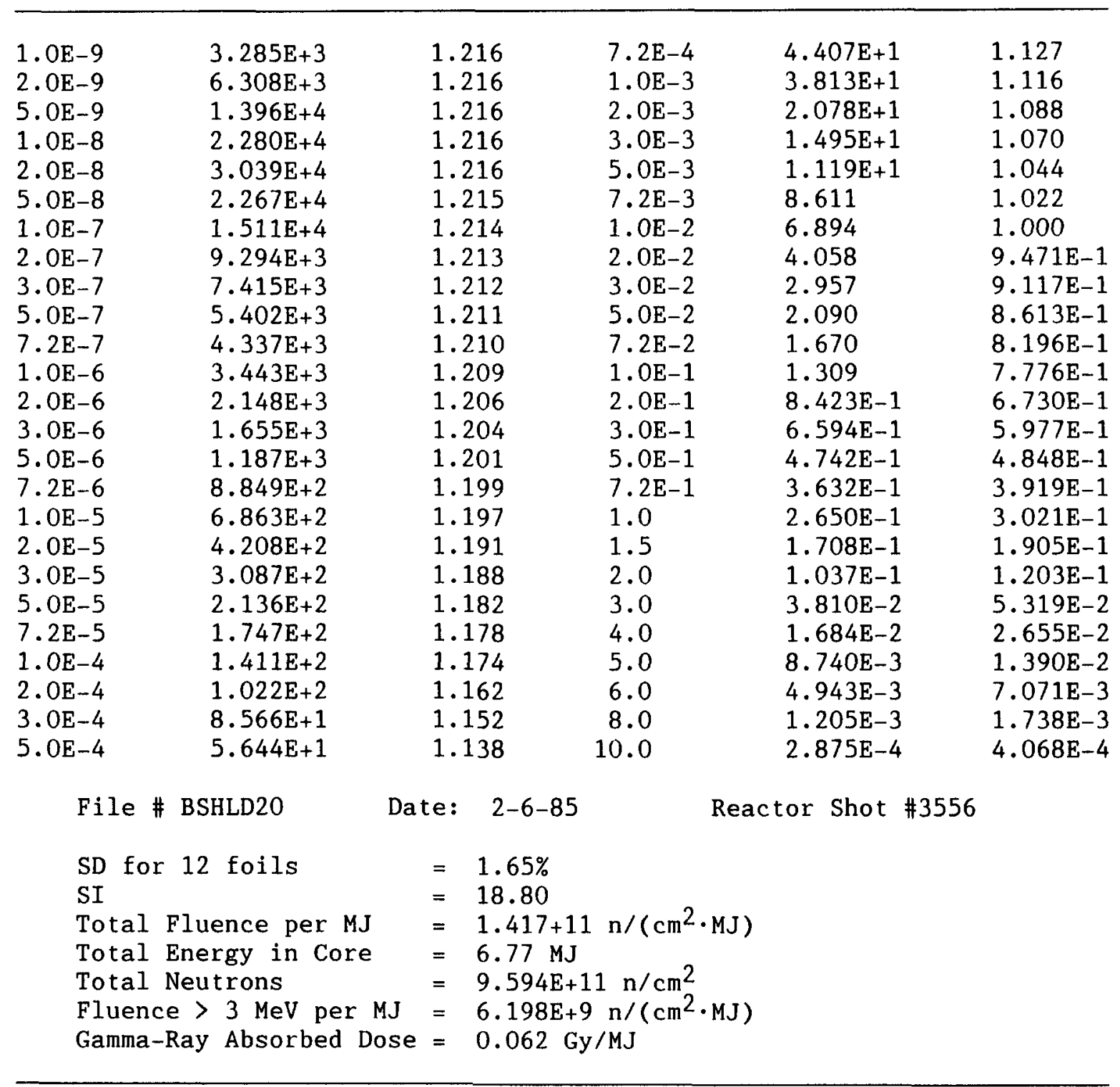




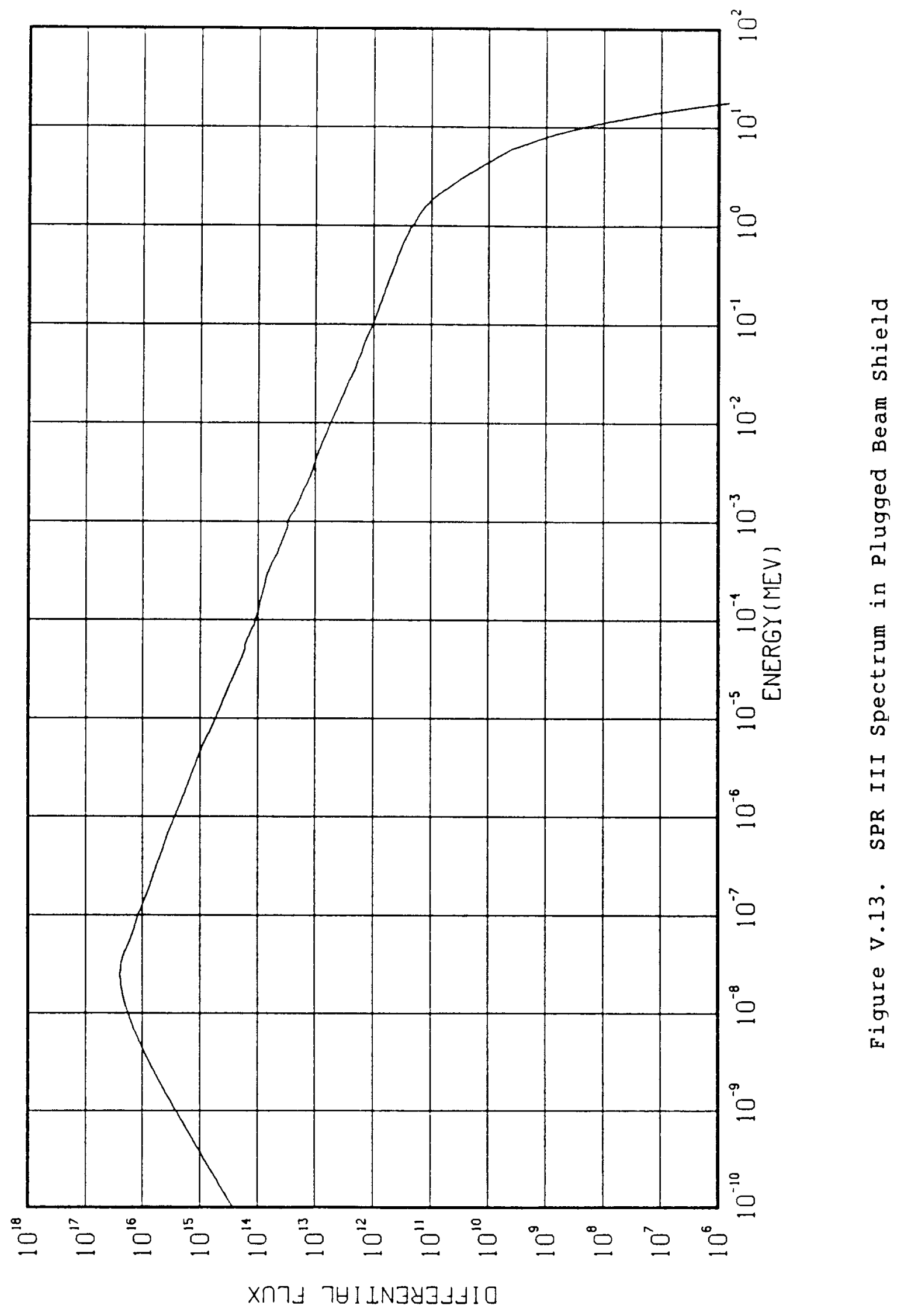


(8) SPR II Leakage Spectrum Inside Beam Shield Aperture (BESHLD 32)

The configuration is the same as that shown in Figure V.12, except that the foil set was placed in the open aperture. There were two exposures--one with the fission foils inside the boron ball and the other with the rest of the foil set. In the second instance, a number of foils were placed together in groups or in the same cadmium cover because of the limited room in the beam. Figure V.14 shows the foil arrangement inside the aperture.

The foil set exposed is listed in Table V.15, and the ones not marked by asterisks were used in the adjustment process. This latter set included one more foil, Fe54, than was used in the plugged beam shield (Case 7). The reactor pulses were large, and good exposures were obtained of all of the foils. The SI was 8.8. The spectral characteristics are listed in Table V.16, and the spectrum is shown in Figure V.15.
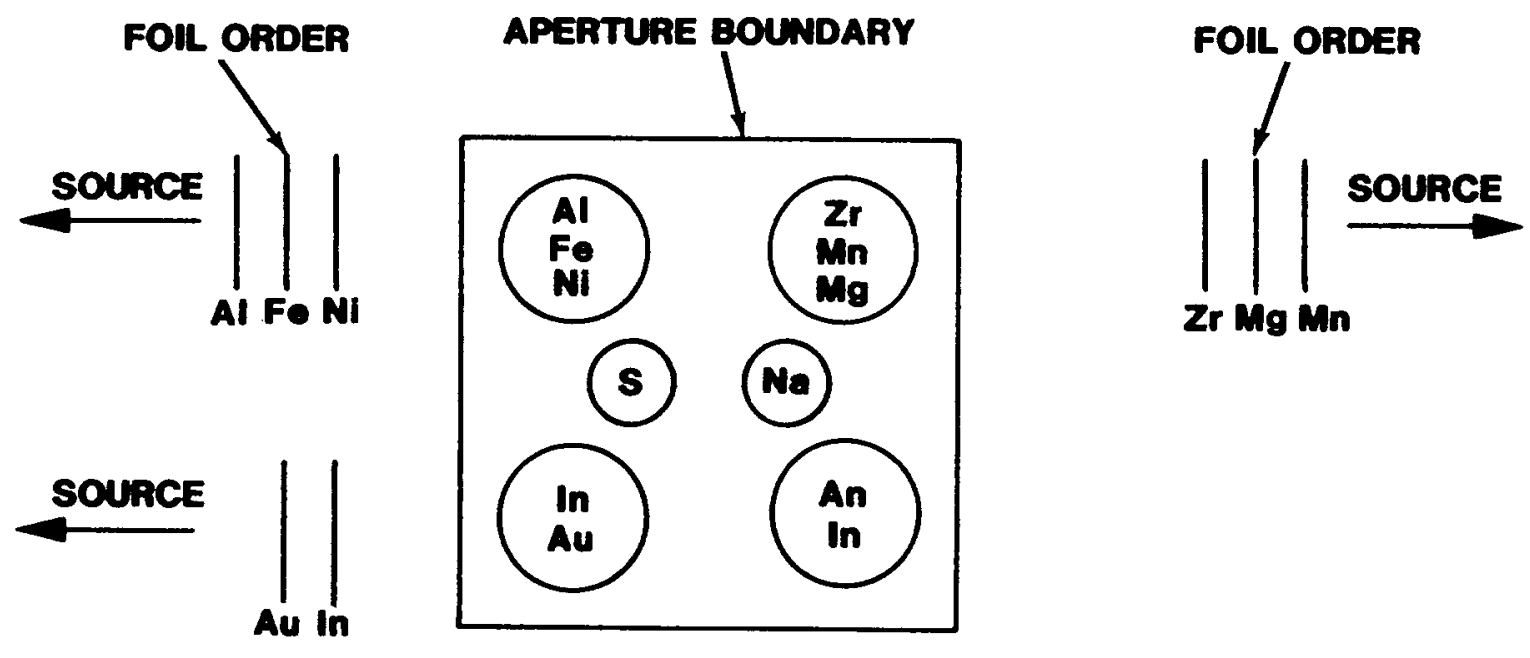

Figure V.14. Foil Arrangement in Beam Shield Aperture 
Table V.15

Foil Activities from SPR III Leakage spectrum in Beam Shield Aperture

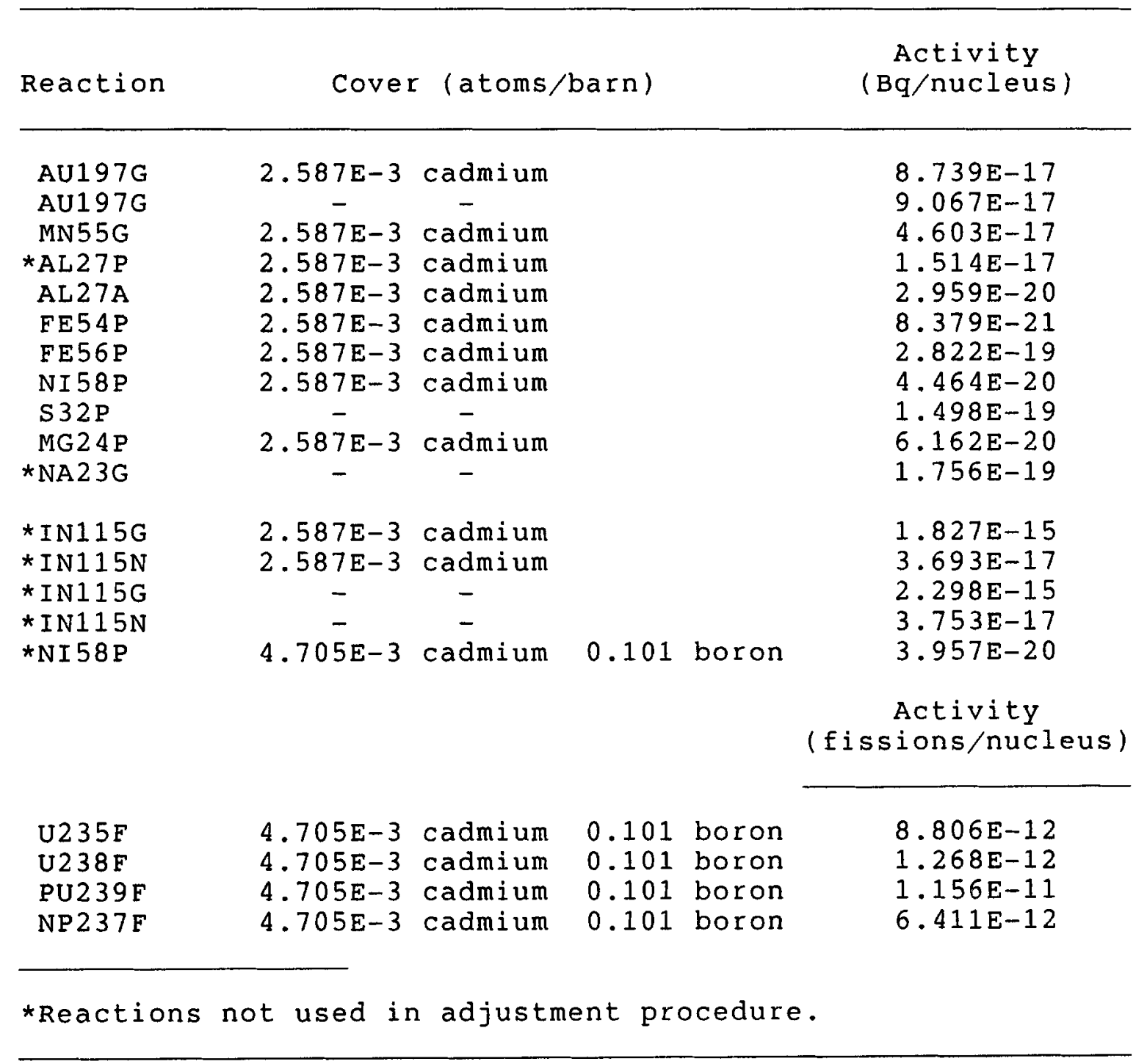


Table V.16

SPR III Leakage Spectrum in Beam Shield Aperture

$\begin{array}{lccccc}\text { E (MeV) } & \text { Differential } & \text { Integral } & \mathrm{E}(\mathrm{MeV}) & \text { Differential } & \text { Integral } \\ & & & & & \\ 1.0 \mathrm{E}-9 & 1.581 \mathrm{E}+3 & 1.079 & 7.2 \mathrm{E}-4 & 1.368 \mathrm{E}+1 & 1.049 \\ 2.0 \mathrm{E}-9 & 3.036 \mathrm{E}+3 & 1.079 & 1.0 \mathrm{E}-3 & 1.113 \mathrm{E}+1 & 1.045 \\ 5.0 \mathrm{E}-9 & 6.720 \mathrm{E}+3 & 1.079 & 2.0 \mathrm{E}-3 & 7.591 & 1.036 \\ 1.0 \mathrm{E}-8 & 1.097 \mathrm{E}+4 & 1.079 & 3.0 \mathrm{E}-3 & 6.067 & 1.029 \\ 2.0 \mathrm{E}-8 & 1.463 \mathrm{E}+4 & 1.079 & 5.0 \mathrm{E}-3 & 4.567 & 1.019 \\ 5.0 \mathrm{E}-8 & 1.096 \mathrm{E}+4 & 1.079 & 7.2 \mathrm{E}-3 & 3.654 & 1.009 \\ 1.0 \mathrm{E}-7 & 7.265 \mathrm{E}+3 & 1.078 & 1.0 \mathrm{E}-2 & 3.080 & 1.000 \\ 2.0 \mathrm{E}-7 & 4.369 \mathrm{E}+3 & 1.078 & 2.0 \mathrm{E}-2 & 2.106 & 9.746 \mathrm{E}-1 \\ 3.0 \mathrm{E}-7 & 3.447 \mathrm{E}+3 & 1.077 & 3.0 \mathrm{E}-2 & 1.668 & 9.556 \mathrm{E}-1 \\ 5.0 \mathrm{E}-7 & 2.413 \mathrm{E}+3 & 1.077 & 5.0 \mathrm{E}-2 & 1.293 & 9.261 \mathrm{E}-1 \\ 7.2 \mathrm{E}-7 & 1.810 \mathrm{E}+3 & 1.076 & 7.2 \mathrm{E}-2 & 1.105 & 8.997 \mathrm{E}-1 \\ 1.0 \mathrm{E}-6 & 1.458 \mathrm{E}+3 & 1.076 & 1.0 \mathrm{E}-1 & 9.808 \mathrm{E}-1 & 8.703 \mathrm{E}-1 \\ 2.0 \mathrm{E}-6 & 8.787 \mathrm{E}+2 & 1.075 & 2.0 \mathrm{E}-1 & 7.028 \mathrm{E}-1 & 7.875 \mathrm{E}-1 \\ 3.0 \mathrm{E}-6 & 6.394 \mathrm{E}+2 & 1.074 & 3.0 \mathrm{E}-1 & 5.641 \mathrm{E}-1 & 7.239 \mathrm{E}-1 \\ 5.0 \mathrm{E}-6 & 4.599 \mathrm{E}+2 & 1.073 & 5.0 \mathrm{E}-1 & 4.273 \mathrm{E}-1 & 6.250 \mathrm{E}-1 \\ 7.2 \mathrm{E}-6 & 3.440 \mathrm{E}+2 & 1.072 & 7.2 \mathrm{E}-1 & 3.491 \mathrm{E}-1 & 5.390 \mathrm{E}-1 \\ 1.0 \mathrm{E}-5 & 2.614 \mathrm{E}+2 & 1.071 & 1.0 & 2.794 \mathrm{E}-1 & 4.497 \mathrm{E}-1 \\ 2.0 \mathrm{E}-5 & 1.586 \mathrm{E}+2 & 1.069 & 1.5 & 2.161 \mathrm{E}-1 & 3.241 \mathrm{E}-1 \\ 3.0 \mathrm{E}-5 & 1.165 \mathrm{E}+2 & 1.068 & 2.0 & 1.593 \mathrm{E}-1 & 2.273 \mathrm{E}-1 \\ 5.0 \mathrm{E}-5 & 8.120 \mathrm{E}+1 & 1.066 & 3.0 & 7.606 \mathrm{E}-2 & 1.141 \mathrm{E}-1 \\ 7.2 \mathrm{E}-5 & 6.267 \mathrm{E}+1 & 1.064 & 4.0 & 3.664 \mathrm{E}-2 & 5.910 \mathrm{E}-2 \\ 1.0 \mathrm{E}-4 & 4.890 \mathrm{E}+1 & 1.062 & 5.0 & 2.057 \mathrm{E}-2 & 3.126 \mathrm{E}-2 \\ 2.0 \mathrm{E}-4 & 2.923 \mathrm{E}+1 & 1.059 & 6.0 & 1.329 \mathrm{E}-2 & 1.407 \mathrm{E}-2 \\ 3.0 \mathrm{E}-4 & 2.298 \mathrm{E}+1 & 1.056 & 8.0 & 1.788 \mathrm{E}-3 & 2.576 \mathrm{E}-3 \\ 5.0 \mathrm{E}-4 & 1.679 \mathrm{E}+1 & 1.052 & 10.0 & 4.264 \mathrm{E}-4 & 6.058 \mathrm{E}-4\end{array}$

$\begin{array}{ll}\text { File \# BESHLD32 Date: } & 2-7-85 \quad \text { Reactor Shot \#3557 } \\ & =2.00 \% \\ \text { SD for } 13 \text { foils } & =8.76 \\ \text { SI } & =8.154 \mathrm{E}+11 \mathrm{n} /\left(\mathrm{cm}^{2} \cdot \mathrm{MJ}\right) \\ \text { Total Fluence per MJ } & 9.57 \mathrm{MJ} \\ \text { Total Energy in Core } & 7.803 \mathrm{E}+12 \mathrm{n} / \mathrm{cm}^{2} \\ \text { Total Neutrons } & 8.619 \mathrm{E}+10 \mathrm{n} /\left(\mathrm{cm}^{2} \cdot \mathrm{MJ}\right) \\ \text { Fluence }>\text { MeV per MJ } & =1.126 \mathrm{~Gy} / \mathrm{MJ}\end{array}$




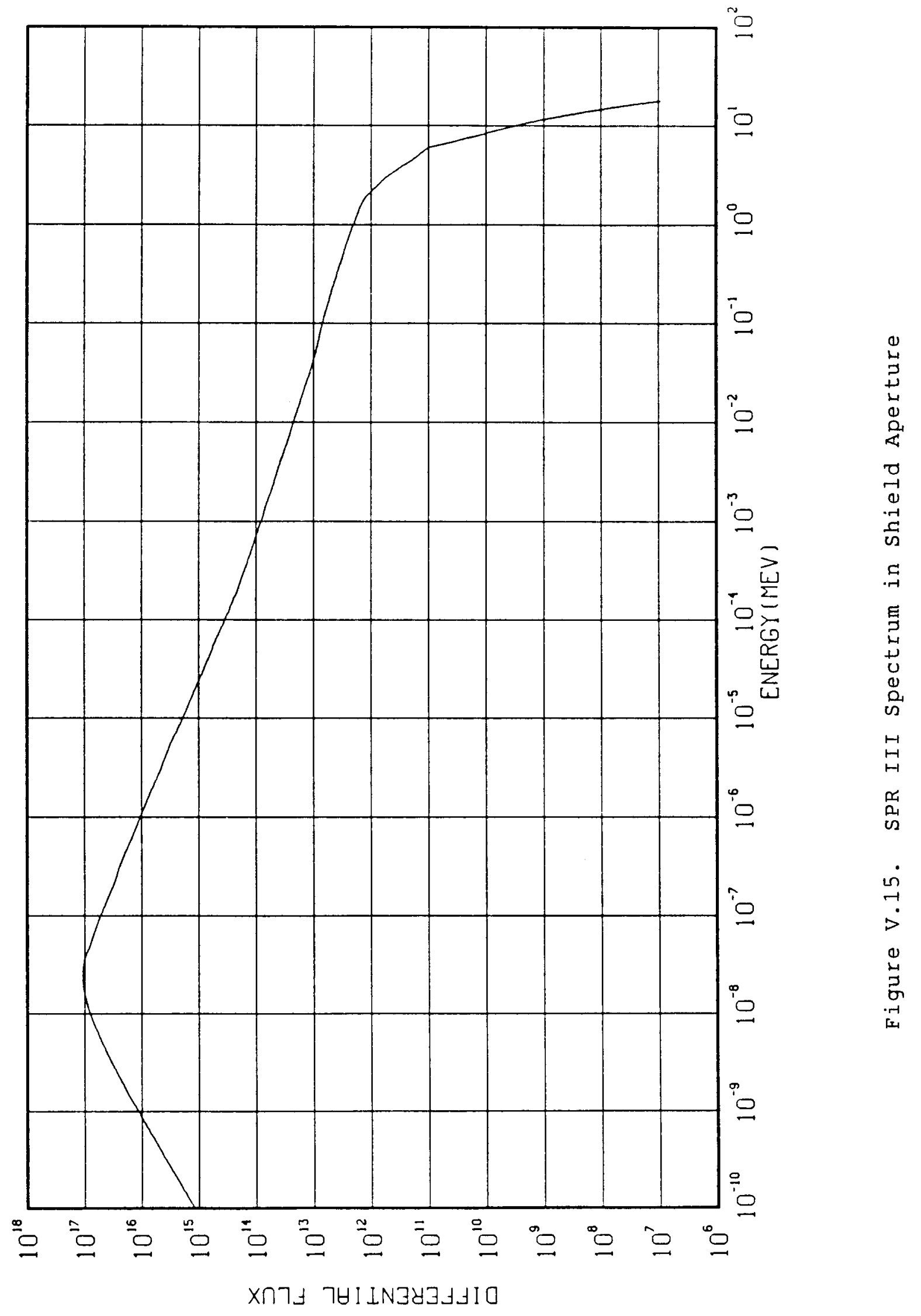




\section{(9) White Sands Missile Range FBR Leakage spectrum (WHSL25)}

Two sets of foils were exposed on opposite sides of the FBR during the same pulse. Unfortunately, the usual aluminum foil data were not available, and the Fe54 reaction was not successfully read. In the determination of the activities (measured in SNLA's counting laboratory), the corresponding reactions from the opposite sides of the reactor (each $61 \mathrm{~cm}$ from core centerline) were averaged together. The configurations of the foil sets are shown in Figure v.16. The foil reactions are listed in Table V.17; those marked with asterisks were not used in the adjustment procedure for reasons that have been discussed earlier in the chapter. The spectrum characteristics are listed in Table V.18, and the spectrum is plotted in Figure V.17. We have not yet been able to find as smooth a spectrum for this foil set as usual, and some inappropriate structure shows in the plot. Most of it is caused by some disagreement between the manganese and sodium reactions. WSMR personnel later confirmed that the fluence along the vertical plane may have varied by $17 \%$. Figure V.16 indicates that the manganese foil, being nearer the vertical axis, may have been in a higher fluence than the sodium.

The SI obtained is 6.65 , lower than the 7.6 of the SPR III leakage spectrum at 17 inches. The dip at $1 \mathrm{E}-2 \mathrm{MeV}$ and the hump at $2 \mathrm{E}-1 \mathrm{MeV}$ are caused by fitting the sodium and plutonium reactions in this energy region. Since the Na23(n,r)Na24 reaction activity appears to be low in this case, the structure seen in this result may be exaggerated. 

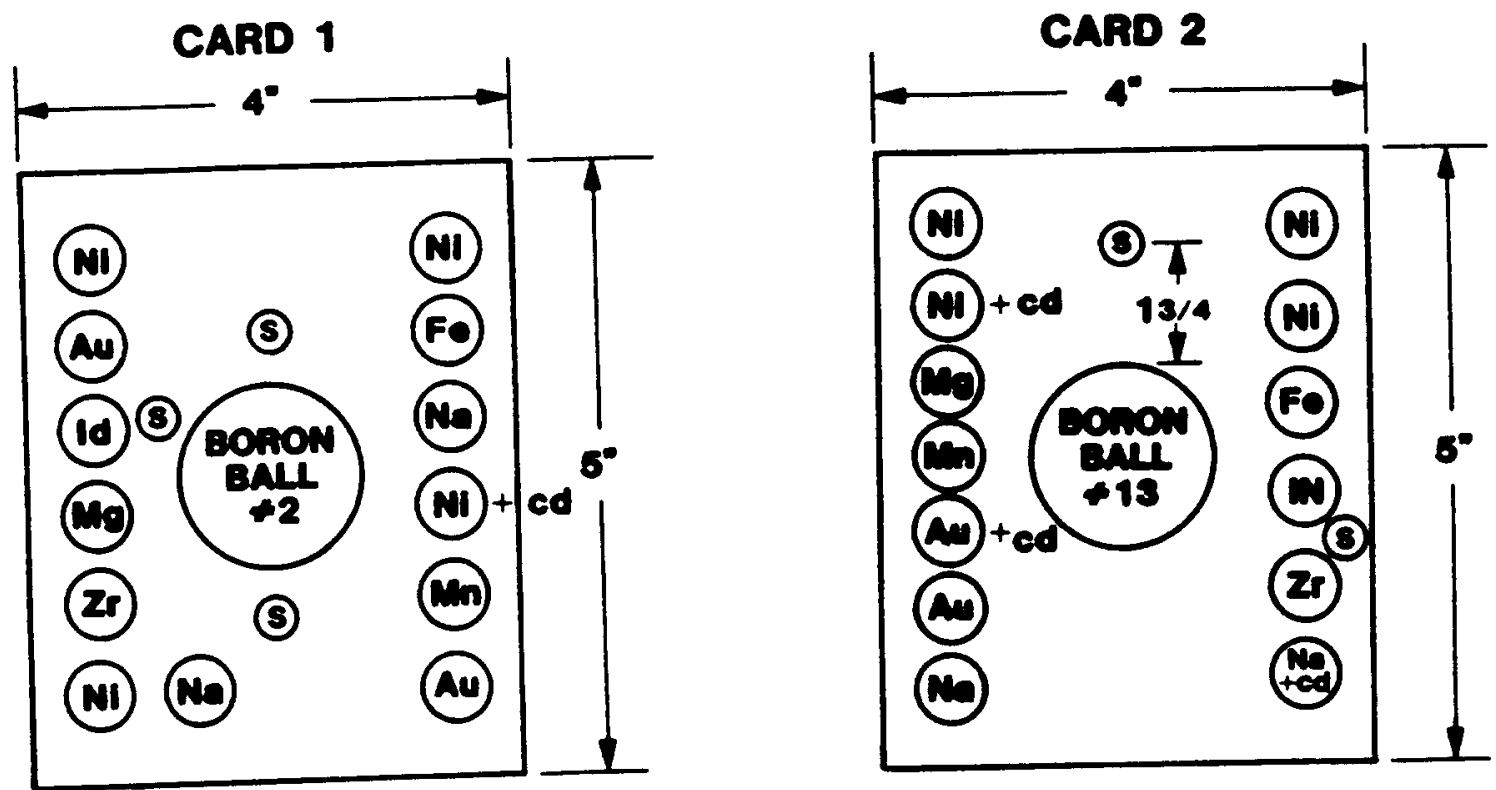

Figure V.16. Foil set Arrangements at WSMR FBR 
Table V.17

Foil Activities for WSMR FBR Leakage spectrum

\begin{tabular}{|c|c|c|c|c|}
\hline Reaction & \multicolumn{3}{|c|}{ over (atoms/barn) } & $\begin{array}{c}\text { Activity } \\
\text { (Bq/nucleus) }\end{array}$ \\
\hline $\begin{array}{l}\text { AU197G } \\
\text { AU197G } \\
\text { MN55G } \\
\text { NI58P } \\
\text { S32P } \\
\text { MG24P } \\
\text { FE56P } \\
\text { *FE54P } \\
\text { NA23G } \\
\text { NA23G }\end{array}$ & $\begin{array}{c}2.587 \mathrm{E}-3 \\
- \\
2.587 \mathrm{E}-3 \\
2.587 \mathrm{E}-3 \\
- \\
2.587 \mathrm{E}-3 \\
2.587 \mathrm{E}-3 \\
2.587 \mathrm{E}-3 \\
4.705 \mathrm{E}-3 \\
-\end{array}$ & $\begin{array}{l}\text { cadmium } \\
- \\
\text { cadmium } \\
\text { cadmium } \\
- \\
\text { cadmium } \\
\text { cadmium } \\
\text { cadmium } \\
\text { cadmium } \\
-\end{array}$ & 0.101 boron & $\begin{array}{l}6.463 \mathrm{E}-17 \\
8.579 \mathrm{E}-17 \\
2.311 \mathrm{E}-17 \\
5.906 \mathrm{E}-20 \\
2.076 \mathrm{E}-19 \\
7.695 \mathrm{E}-20 \\
3.921 \mathrm{E}-19 \\
1.293 \mathrm{E}-20 \\
1.057 \mathrm{E}-19 \\
4.032 \mathrm{E}-19\end{array}$ \\
\hline $\begin{array}{l}\text { * IN115G } \\
\text { *IN115N } \\
\text { *NI } 58 \mathrm{P} \\
\text { *NI58P }\end{array}$ & $\begin{array}{c}2.587 \mathrm{E}-3 \\
2.587 \mathrm{E}-3 \\
4.705 \mathrm{E}-3 \\
-\end{array}$ & $\begin{array}{l}\text { cadmium } \\
\text { cadmium } \\
\text { cadmium } \\
\quad-\end{array}$ & 0.101 boron & $\begin{array}{c}1.415 \mathrm{E}-15 \\
4.543 \mathrm{E}-17 \\
5.620 \mathrm{E}-20 \\
5.981 \mathrm{E}-20 \\
\text { Activity } \\
\text { (fissions/nucleus) }\end{array}$ \\
\hline $\begin{array}{l}\text { U2 } 35 F \\
\text { U238F } \\
\text { PU239F } \\
\text { NP237F }\end{array}$ & $\begin{array}{l}4.705 E-3 \\
4.705 E-3 \\
4.705 E-3 \\
4.705 E-3\end{array}$ & $\begin{array}{l}\text { cadmium } \\
\text { cadmium } \\
\text { cadmium } \\
\text { cadmium }\end{array}$ & $\begin{array}{l}0.101 \text { boron } \\
0.101 \text { boron } \\
0.101 \text { boron } \\
0.101 \text { boron }\end{array}$ & $\begin{array}{l}8.693 \mathrm{E}-12 \\
1.343 \mathrm{E}-12 \\
1.184 \mathrm{E}-11 \\
6.467 \mathrm{E}-12\end{array}$ \\
\hline
\end{tabular}


Table V.18

WSMR FBR Leakage spectrum

$($ normalized to $\Phi(E>10 \mathrm{keV})=1.00)$

$\begin{array}{lccccc}\text { E (MeV) } & \text { Differential } & \text { Integral } & \mathrm{E}(\mathrm{MeV}) & \text { Differential } & \text { Integral } \\ & & & & & \\ 1.0 \mathrm{E}-9 & 7.816 \mathrm{E}+3 & 1.032 & 7.2 \mathrm{E}-4 & 3.982 & 1.008 \\ 2.0 \mathrm{E}-9 & 1.501 \mathrm{E}+4 & 1.032 & 1.0 \mathrm{E}-3 & 3.169 & 1.007 \\ 5.0 \mathrm{E}-9 & 3.322 \mathrm{E}+4 & 1.032 & 2.0 \mathrm{E}-3 & 1.298 & 1.005 \\ 1.0 \mathrm{E}-8 & 5.423 \mathrm{E}+4 & 1.032 & 3.0 \mathrm{E}-3 & 8.699 \mathrm{E}-1 & 1.004 \\ 2.0 \mathrm{E}-8 & 7.223 \mathrm{E}+4 & 1.032 & 5.0 \mathrm{E}-3 & 5.934 \mathrm{E}-1 & 1.002 \\ 5.0 \mathrm{E}-8 & 5.348 \mathrm{E}+4 & 1.030 & 7.2 \mathrm{E}-3 & 4.709 \mathrm{E}-1 & 1.001 \\ 1.0 \mathrm{E}-7 & 2.679 \mathrm{E}+4 & 1.028 & 1.0 \mathrm{E}-2 & 4.070 \mathrm{E}-1 & 1.000 \\ 2.0 \mathrm{E}-7 & 1.336 \mathrm{E}+4 & 1.026 & 2.0 \mathrm{E}-2 & 3.609 \mathrm{E}-1 & 9.962 \mathrm{E}-1 \\ 3.0 \mathrm{E}-7 & 7.843 \mathrm{E}+3 & 1.025 & 3.0 \mathrm{E}-2 & 3.867 \mathrm{E}-1 & 9.925 \mathrm{E}-1 \\ 5.0 \mathrm{E}-7 & 4.075 \mathrm{E}+3 & 1.023 & 5.0 \mathrm{E}-2 & 5.269 \mathrm{E}-1 & 9.835 \mathrm{E}-1 \\ 7.2 \mathrm{E}-7 & 2.466 \mathrm{E}+3 & 1.023 & 7.2 \mathrm{E}-2 & 7.315 \mathrm{E}-1 & 9.700 \mathrm{E}-1 \\ 1.0 \mathrm{E}-6 & 1.728 \mathrm{E}+3 & 1.022 & 1.0 \mathrm{E}-1 & 9.778 \mathrm{E}-1 & 9.462 \mathrm{E}-1 \\ 2.0 \mathrm{E}-6 & 7.778 \mathrm{E}+2 & 1.021 & 2.0 \mathrm{E}-1 & 1.073 & 8.424 \mathrm{E}-1 \\ 3.0 \mathrm{E}-6 & 5.330 \mathrm{E}+2 & 1.020 & 3.0 \mathrm{E}-1 & 9.046 \mathrm{E}-1 & 7.423 \mathrm{E}-1 \\ 5.0 \mathrm{E}-6 & 3.564 \mathrm{E}+2 & 1.019 & 5.0 \mathrm{E}-1 & 5.624 \mathrm{E}-1 & 5.960 \mathrm{E}-1 \\ 7.2 \mathrm{E}-6 & 2.386 \mathrm{E}+2 & 1.019 & 7.2 \mathrm{E}-1 & 3.060 \mathrm{E}-1 & 5.026 \mathrm{E}-1 \\ 1.0 \mathrm{E}-5 & 1.736 \mathrm{E}+2 & 1.018 & 1.0 & 2.177 \mathrm{E}-1 & 4.283 \mathrm{E}-1 \\ 2.0 \mathrm{E}-5 & 9.196 \mathrm{E}+1 & 1.017 & 1.5 & 1.642 \mathrm{E}-1 & 3.316 \mathrm{E}-1 \\ 3.0 \mathrm{E}-5 & 6.430 \mathrm{E}+1 & 1.016 & 2.0 & 1.321 \mathrm{E}-1 & 2.564 \mathrm{E}-1 \\ 5.0 \mathrm{E}-5 & 4.407 \mathrm{E}+1 & 1.015 & 3.0 & 8.118 \mathrm{E}-2 & 1.503 \mathrm{E}-1 \\ 7.2 \mathrm{E}-5 & 3.137 \mathrm{E}+1 & 1.014 & 4.0 & 4.988 \mathrm{E}-2 & 8.503 \mathrm{E}-2 \\ 1.0 \mathrm{E}-4 & 2.389 \mathrm{E}+1 & 1.013 & 5.0 & 3.058 \mathrm{E}-2 & 4.497 \mathrm{E}-2 \\ 2.0 \mathrm{E}-4 & 1.314 \mathrm{E}+1 & 1.012 & 6.0 & 2.051 \mathrm{E}-2 & 1.878 \mathrm{E}-2 \\ 3.0 \mathrm{E}-4 & 9.907 & 1.011 & 8.0 & 2.004 \mathrm{E}-3 & 2.904 \mathrm{E}-3 \\ 5.0 \mathrm{E}-4 & 5.728 & 1.009 & 10.0 & 4.830 \mathrm{E}-4 & 6.850 \mathrm{E}-4\end{array}$

File \# WHSL25

Date: $8-9-85$

Reactor Shot \# 11044

SD for 13 foils

SI

$=2.50 \%$

$=6.65$

Total Fluence per MJ

Total Energy in Core

$=8.154 \mathrm{E}+11 \mathrm{n} /\left(\mathrm{cm}^{2} \cdot \mathrm{MJ}\right)$

Total Neutrons

$=9.60 \mathrm{MJ}$

$=7.828 \mathrm{E}+12 \mathrm{n} / \mathrm{cm}^{2}$

Fluence $>3 \mathrm{MeV}$ per $\mathrm{MJ}=1.188 \mathrm{E}+11 \mathrm{n} /\left(\mathrm{cm}^{2} \cdot \mathrm{MJ}\right)$

Gamma-Ray Absorbed Dose $=$ not measured 


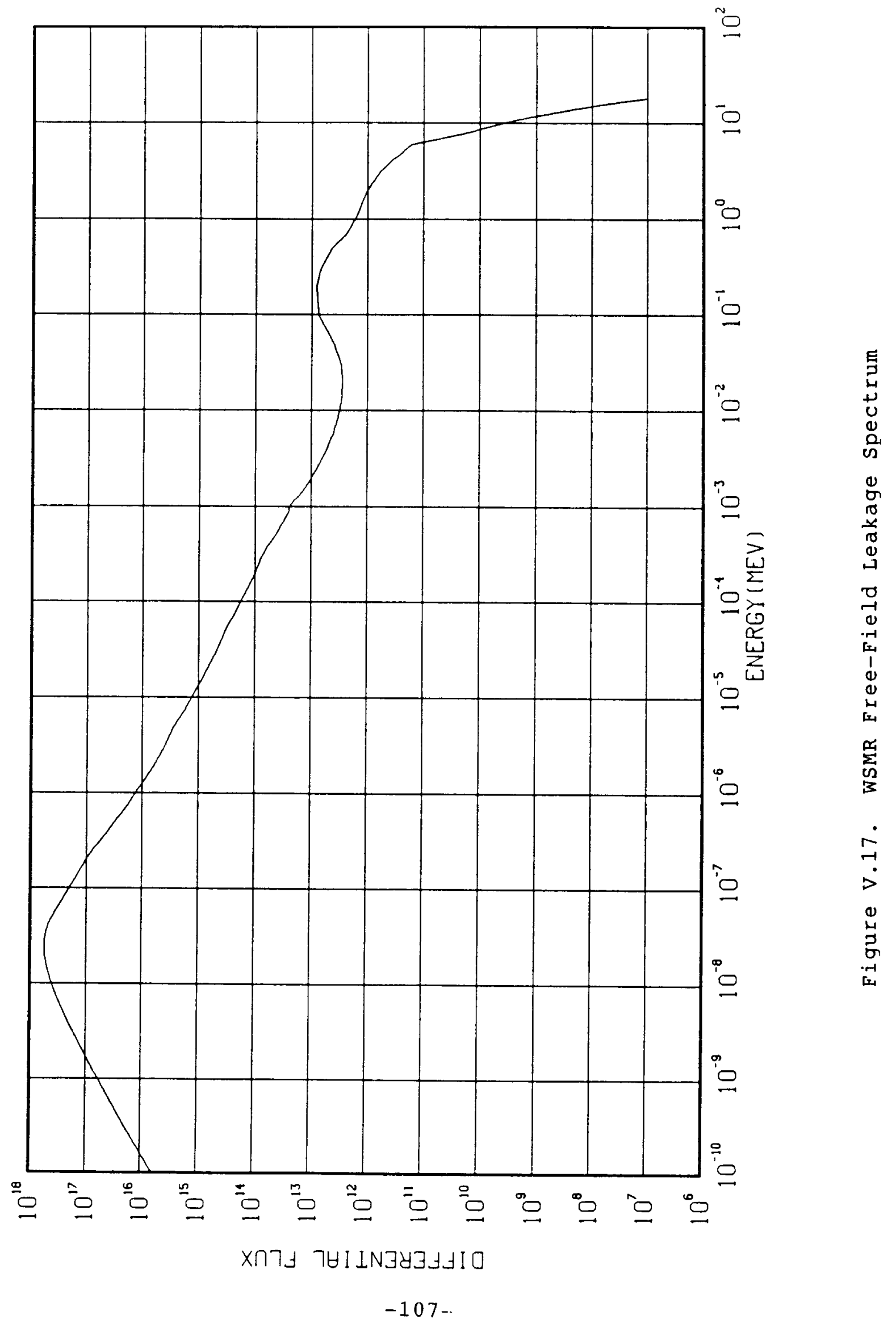




\section{(10) ACRR Central Cavity in Free Field (ACRRCF28)}

The ACRR is a water-moderated, pulsed reactor of the TRIGA Type.[25] The central experimental cavity is a dry, steel-walled vessel with an inside diameter of $23 \mathrm{~cm}$. The foil sets were exposed in the same manner as shown in Figure II.1. In this case, the rings of foils on each side of the vertical flux centerline were $15 \mathrm{~cm}$ in diameter.

Because of the additional room available in this cavity, full foil sets were exposed with and without the boron ball. The foils sensitive at high energies exhibited the same activities with or without the presence of the boron ball. Those sensitive at low energies were shadowed by about $4 \%$. In any case, only the activities obtained in the absence of the ball were used in the adjustment procedure (except, of course, for the fission foils). The list of foils exposed is given in Table V.19. Those used in the adjustment procedure are not marked by asterisks. The list of reactions used in this experiment is more extensive than that of SPR III, not only because the cavity is larger, but also because the exposures were approximately an order of magnitude higher. Thus, additional reactions could be read with adequate statistics, and the reactions were read more quickly. The runs were made in the steady-state mode at $50 \mathrm{~kW}$ for $200 \mathrm{~s}$.

As has been the practice for this work, the In115(n, n') In115, In115(n,r)In116, A127(n,p)Mg27, and Ni58(n,p)Co58 reactions (inside the boron ball) were all left out of the adjustment procedure because of the reasons cited previously. However, two additional reactions not previously attempted were also deleted--Fe58(n,r)Fe59 and $\operatorname{Cu} 63(n, r)$ Cu59. The Fe58 simply was in serious disagreement with the others, and the copper activity, as determined from the two gamma-ray peaks, was in disagreement when either $\gamma$-ray line was used in the analysis. We suspect that errors exist in the cross-section library or in the counting program's handling of these reactions.

The spectrum characteristics are listed in Table V.20, and the differential fluence versus energy is shown in Figure V.18. The degradation of the neutron energy caused by the moderation in the water is evident in the shape of this spectrum, and the SI is a high 12.14. We found the activities of this foil set very easy to fit with a smooth spectrum that is simple in structure. In this case, deletion of the indium and aluminum reactions made little difference in the shape of the spectrum. 
Table V.19

Foil Activities for ACRR Bare Cavity spectrum

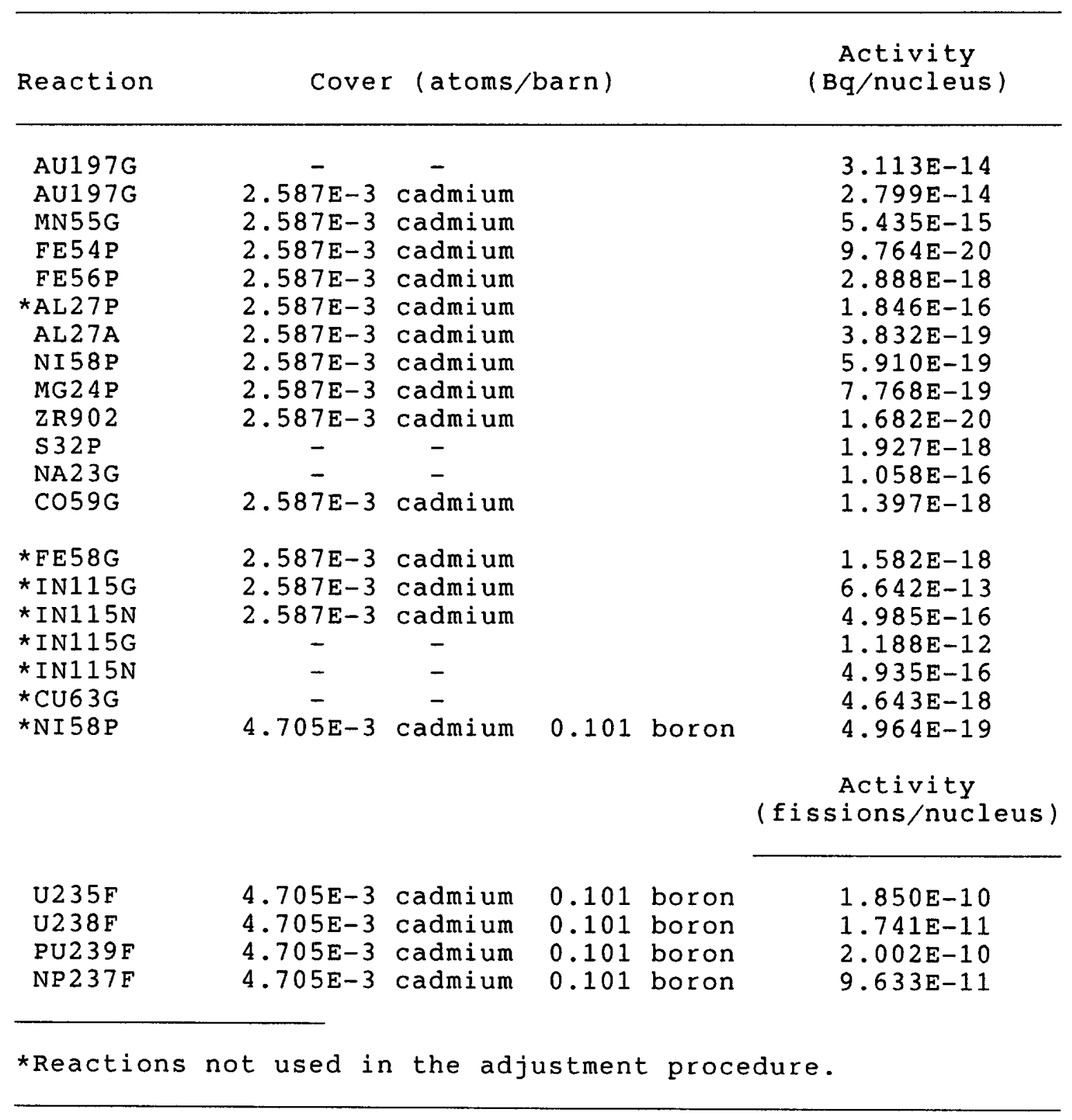


Table V.20

ACRR Free-Field Cavity spectrum

\begin{tabular}{lccccc}
\hline E (MeV) & Differential & Integral & $\mathrm{E}(\mathrm{MeV})$ & Differential & Integral \\
& & & & & \\
\hline $1.0 \mathrm{E}-9$ & $1.225 \mathrm{E}+5$ & 1.579 & $7.2 \mathrm{E}-4$ & $5.427 \mathrm{E}+1$ & 1.134 \\
$2.0 \mathrm{E}-9$ & $2.352 \mathrm{E}+5$ & 1.579 & $1.0 \mathrm{E}-3$ & $3.999 \mathrm{E}+1$ & 1.120 \\
$5.0 \mathrm{E}-9$ & $5.206 \mathrm{E}+5$ & 1.578 & $2.0 \mathrm{E}-3$ & $2.314 \mathrm{E}+1$ & 1.089 \\
$1.0 \mathrm{E}-8$ & $8.499 \mathrm{E}+5$ & 1.575 & $3.0 \mathrm{E}-3$ & $1.624 \mathrm{E}+1$ & 1.070 \\
$2.0 \mathrm{E}-8$ & $1.133 \mathrm{E}+6$ & 1.565 & $5.0 \mathrm{E}-3$ & $1.088 \mathrm{E}+1$ & 1.043 \\
$5.0 \mathrm{E}-8$ & $8.396 \mathrm{E}+5$ & 1.533 & $7.2 \mathrm{E}-3$ & 8.454 & 1.021 \\
$1.0 \mathrm{E}-7$ & $4.194 \mathrm{E}+5$ & 1.503 & $1.0 \mathrm{E}-2$ & 6.590 & 1.000 \\
$2.0 \mathrm{E}-7$ & $2.097 \mathrm{E}+5$ & 1.473 & $2.0 \mathrm{E}-2$ & 3.964 & $9.490 \mathrm{E}-1$ \\
$3.0 \mathrm{E}-7$ & $1.408 \mathrm{E}+5$ & 1.456 & $3.0 \mathrm{E}-2$ & 3.022 & $9.139 \mathrm{E}-1$ \\
$5.0 \mathrm{E}-7$ & $8.893 \mathrm{E}+4$ & 1.433 & $5.0 \mathrm{E}-2$ & 2.129 & $8.625 \mathrm{E}-1$ \\
$7.2 \mathrm{E}-7$ & $6.339 \mathrm{E}+4$ & 1.416 & $7.2 \mathrm{E}-2$ & 1.653 & $8.208 \mathrm{E}-1$ \\
$1.0 \mathrm{E}-6$ & $4.661 \mathrm{E}+4$ & 1.401 & $1.0 \mathrm{E}-1$ & 1.340 & $7.785 \mathrm{E}-1$ \\
$2.0 \mathrm{E}-6$ & $2.380 \mathrm{E}+4$ & 1.367 & $2.0 \mathrm{E}-1$ & $7.776 \mathrm{E}-1$ & $6.766 \mathrm{E}-1$ \\
$3.0 \mathrm{E}-6$ & $1.543 \mathrm{E}+4$ & 1.347 & $3.0 \mathrm{E}-1$ & $5.698 \mathrm{E}-1$ & $6.090 \mathrm{E}-1$ \\
$5.0 \mathrm{E}-6$ & $9.239 \mathrm{E}+3$ & 1.323 & $5.0 \mathrm{E}-1$ & $3.845 \mathrm{E}-1$ & $5.143 \mathrm{E}-1$ \\
$7.2 \mathrm{E}-6$ & $6.418 \mathrm{E}+3$ & 1.306 & $7.2 \mathrm{E}-1$ & $2.999 \mathrm{E}-1$ & $4.386 \mathrm{E}-1$ \\
$1.0 \mathrm{E}-5$ & $4.540 \mathrm{E}+3$ & 1.290 & 1.0 & $2.348 \mathrm{E}-1$ & $3.625 \mathrm{E}-1$ \\
$2.0 \mathrm{E}-5$ & $2.058 \mathrm{E}+3$ & 1.260 & 1.5 & $1.697 \mathrm{E}-1$ & $2.595 \mathrm{E}-1$ \\
$3.0 \mathrm{E}-5$ & $1.227 \mathrm{E}+3$ & 1.243 & 2.0 & $1.306 \mathrm{E}-1$ & $1.831 \mathrm{E}-1$ \\
$5.0 \mathrm{E}-5$ & $6.854 \mathrm{E}+2$ & 1.224 & 3.0 & $7.525 \mathrm{E}-2$ & $8.024 \mathrm{E}-2$ \\
$7.2 \mathrm{E}-5$ & $4.682 \mathrm{E}+2$ & 1.212 & 4.0 & $2.804 \mathrm{E}-2$ & $3.067 \mathrm{E}-2$ \\
$1.0 \mathrm{E}-4$ & $3.136 \mathrm{E}+2$ & 1.201 & 5.0 & $8.955 \mathrm{E}-3$ & $1.339 \mathrm{E}-2$ \\
$2.0 \mathrm{E}-4$ & $1.571 \mathrm{E}+2$ & 1.178 & 6.0 & $3.779 \mathrm{E}-3$ & $7.235 \mathrm{E}-3$ \\
$3.0 \mathrm{E}-4$ & $1.038 \mathrm{E}+2$ & 1.165 & 8.0 & $1.528 \mathrm{E}-3$ & $2.221 \mathrm{E}-3$ \\
$5.0 \mathrm{E}-4$ & $7.142 \mathrm{E}+1$ & 1.148 & 10.0 & $3.614 \mathrm{E}-4$ & $5.417 \mathrm{E}-4$ \\
& & & & &
\end{tabular}

File \# ACRRCF28 Date: 10-1-84 Reactor Shot \#2172

$\begin{array}{ll}\text { SD for } 16 \text { foils } & =1.95 \% \\ \text { SI } & =12.46 \\ \text { Total Fluence per MJ } & =2.022 \mathrm{E}+13 \mathrm{n} /\left(\mathrm{cm}^{2} \cdot \mathrm{MJ}\right) \\ \text { Total Energy in Core } & =10.0 \mathrm{MJ} \\ \text { Total Neutrons } & 2.022 \mathrm{E}+14 \mathrm{n} / \mathrm{cm}^{2} \\ \text { Fluence }>3 \mathrm{MeV} \text { per MJ } & =1.027 \mathrm{E}+12 \mathrm{n} /\left(\mathrm{cm}^{2} \cdot \mathrm{MJ}\right) \\ \text { Gamma-Ray Absorbed Dose } & =103 \mathrm{~Gy} / \mathrm{MJ}\end{array}$ 


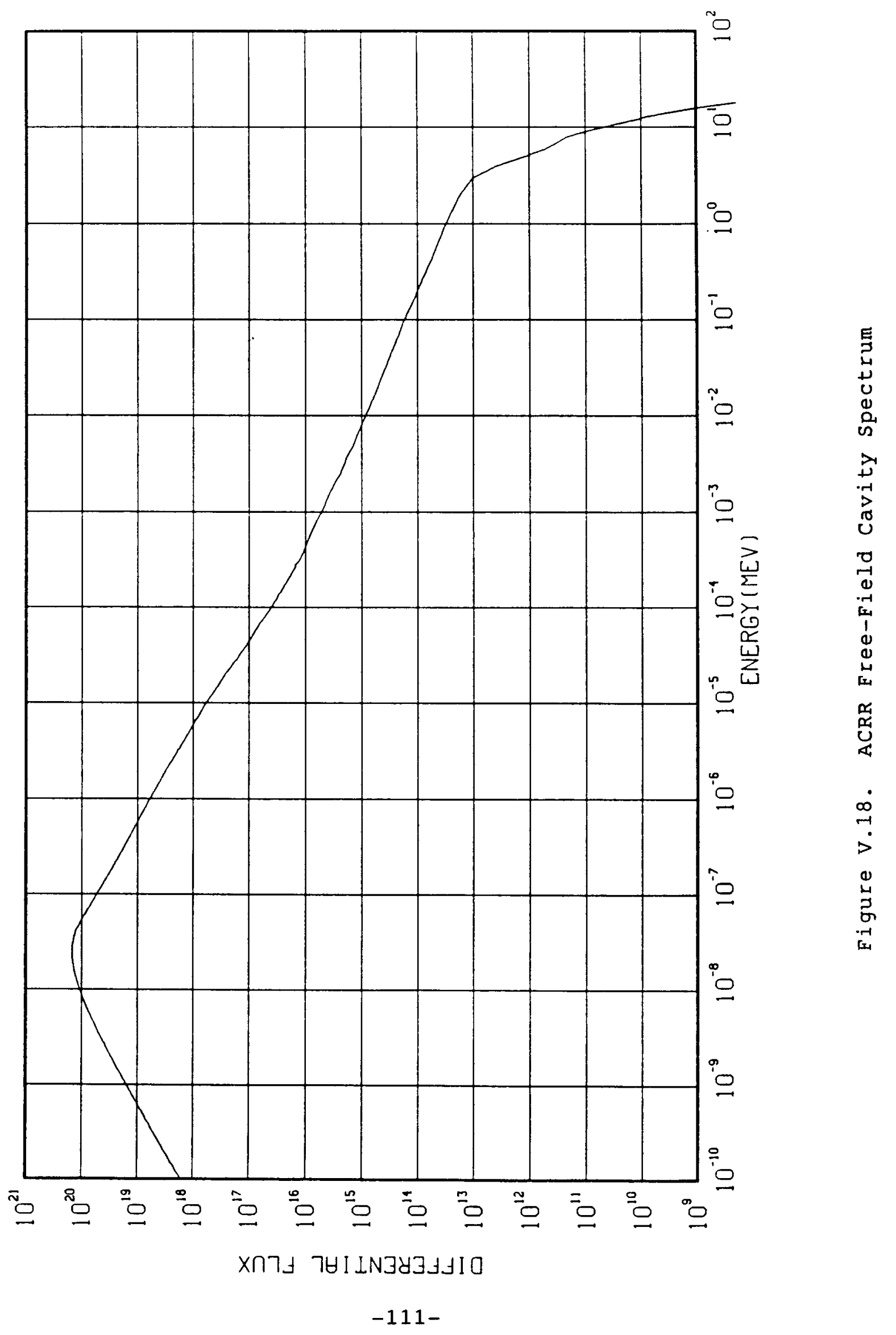




\section{(11) ACRR Central Cavity with Lead and Boron shield ( ACRPBC14)}

Some activity data were made available to us that had been taken inside the lead-and-boron-loaded shield by other experimenters. The shield is shown in Figure V.19. This shield structure was fabricated to reduce the ratio of the gamma-ray fluence to the neutron fluence in the cavity. The boron layer outside the lead liner reduced the thermal neutron fluence. Tests showed that the activation of gold foils was reduced by approximately a factor of two from that seen in the bare cavity. We do not know exactly where the foils were placed inside the shield or whether the boron ball shadowed the foils that had response at low energy. Some delay between exposure and counting led to the loss of activity measurements Al27(n,p)Mg27, A127 $(n, \alpha) N_{2} 24, \quad M g 24(n, p) N a 24, \quad$ and Fe56(n,p)Mn56. The sodium chloride and sulfur pellets, usually included in the set, were absent. Activities obtained from the experiment are listed in Table V.21, and those marked with an asterisk were not used in the adjustment procedure.

The spectrum shown in Figure V.20 is fairly featureless. That may be partially accounted for by the fact that only 9 foils were used in the analysis. The fit was accomplished easily--with an SD of the initial trial of $31 \%$. It dropped to $2.79 \%$ in 2 iterations. It is evident that the lead has degraded the energies of the fast neutrons, because the SI has risen to 16.5. There is some evidence that the boron layer is pulling the spectrum down at thermal energies: the bare and cadmium-covered gold foil activities do not differ by very much. The spectral characteristics are listed in Table V.22. The activity of the nickel in the boron ball included here raised the SI by $0.7 \%$ over that of the unadjusted spectrum without it. 


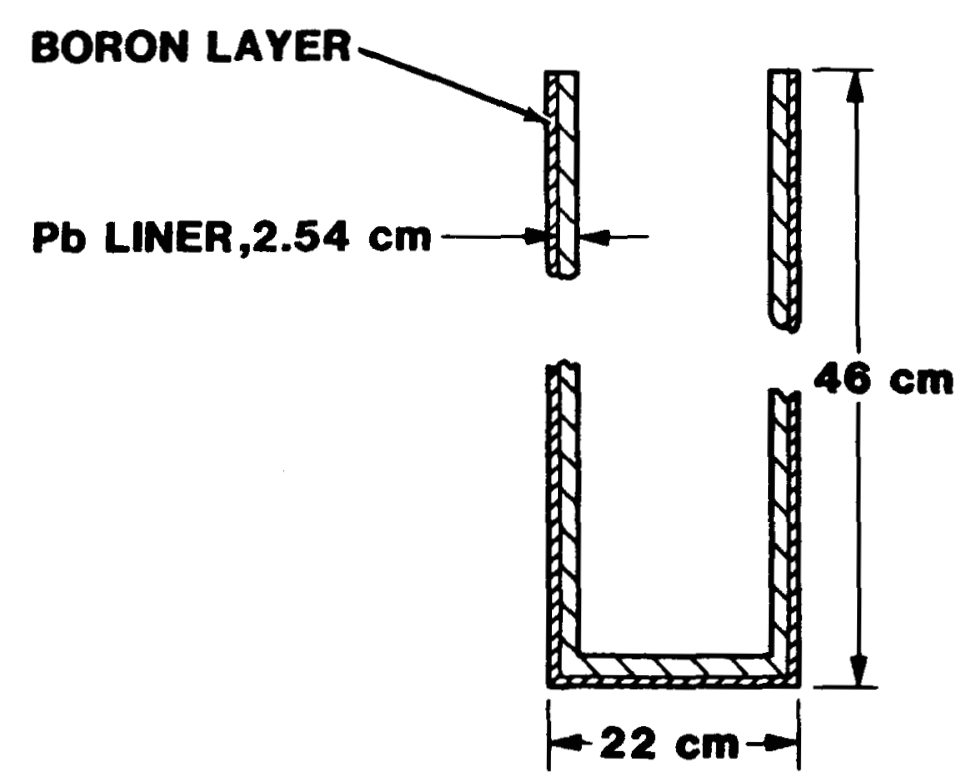

Figure V.19. Lead and Boron Central Cavity Liner for ACRR 
Table V.21

Foil Activities for Old ACRR Lead-Boron-Lined Cavity

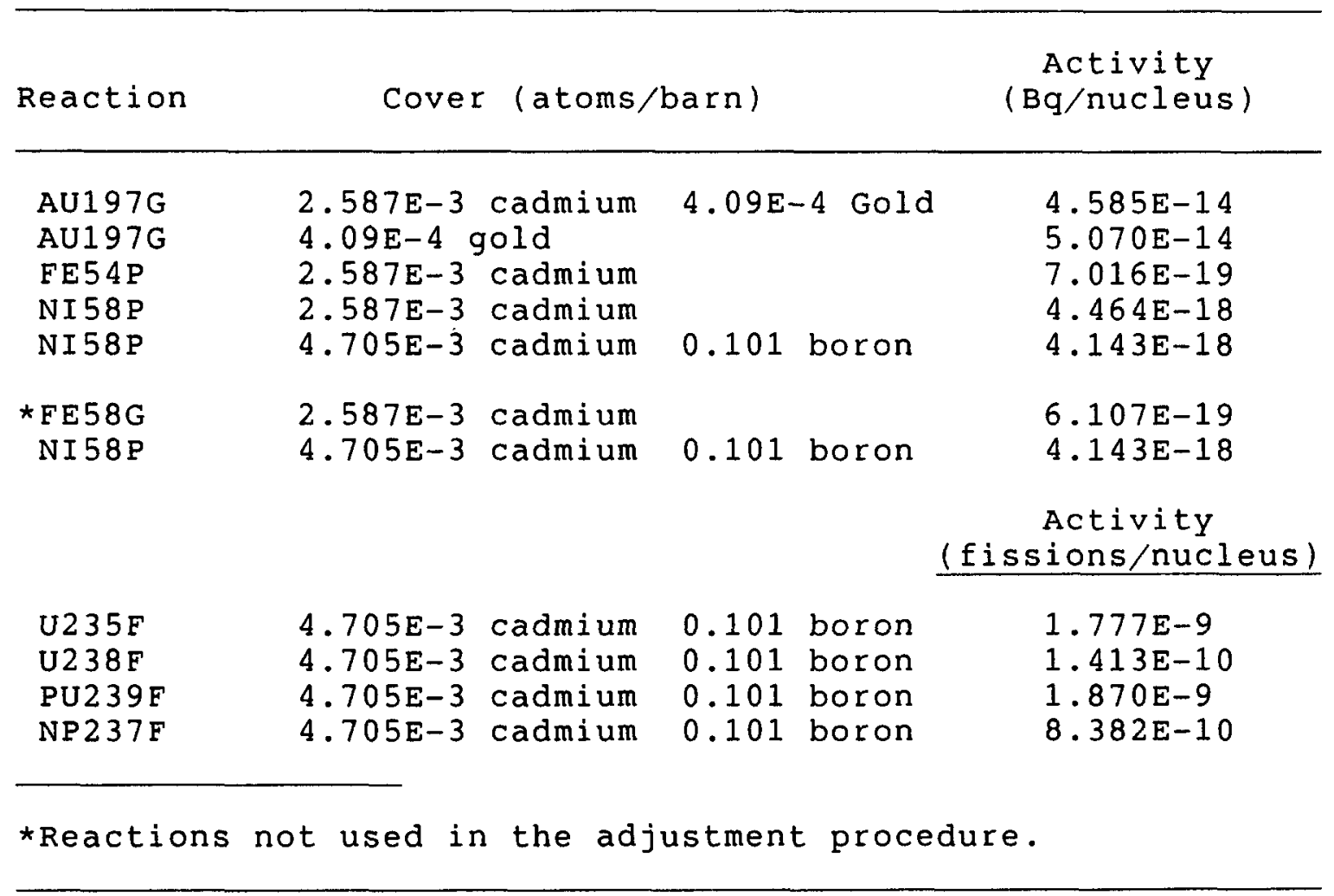




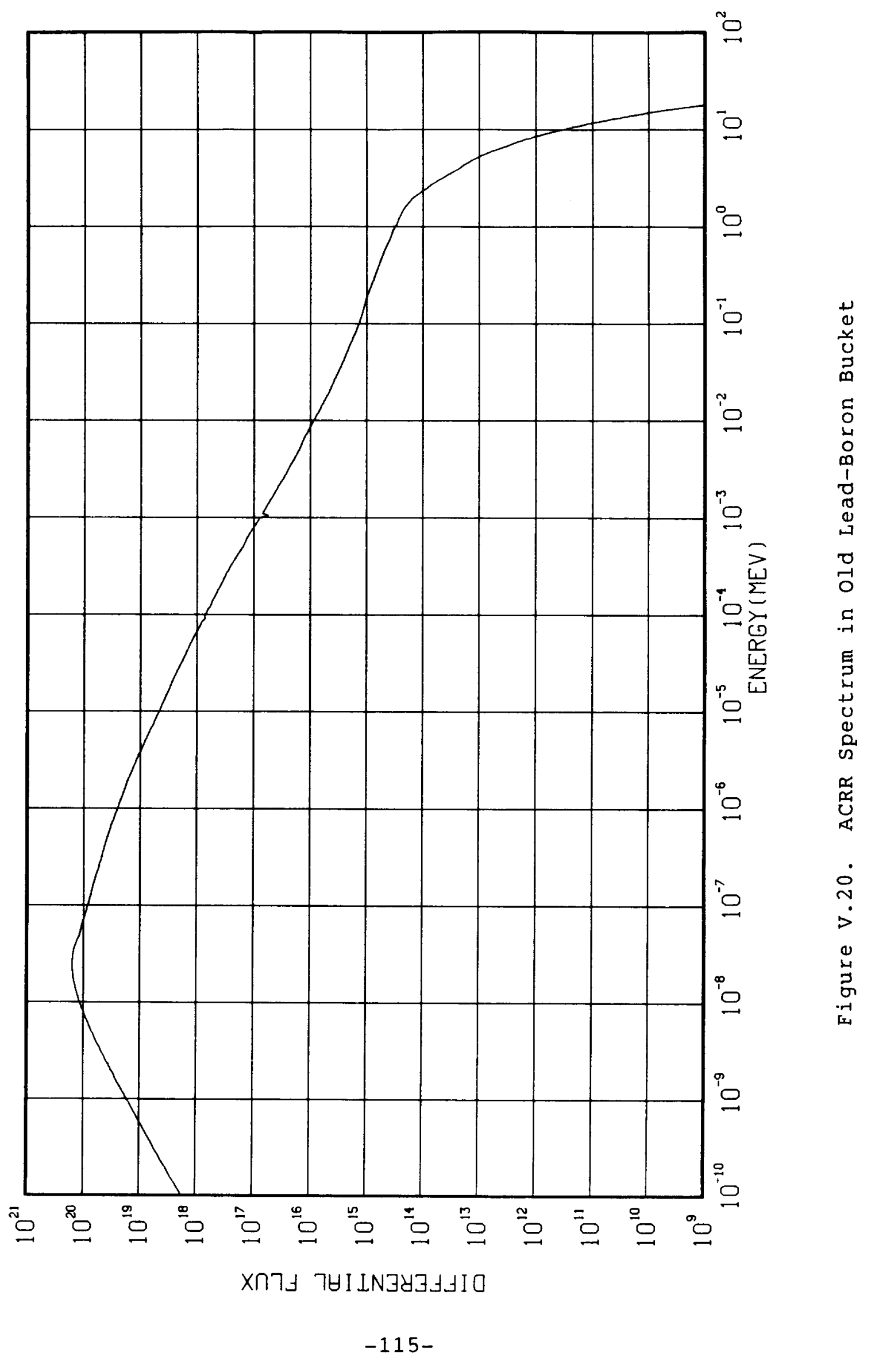


Table V.22

ACRR Central Cavity Spectrum Inside old Lead-Boron Shield

$\begin{array}{lccccc}E(\mathrm{MeV}) & \text { Differential } & \text { Integral } & \mathrm{E}(\mathrm{MeV}) & \text { Differential } & \text { Integral } \\ & & & & & \\ 1.0 \mathrm{E}-9 & 1.430 \mathrm{E}+4 & 1.550 & 7.2 \mathrm{E}-4 & 9.518 \mathrm{E}+1 & 1.182 \\ 2.0 \mathrm{E}-9 & 2.747 \mathrm{E}+4 & 1.550 & 1.0 \mathrm{E}-3 & 6.626 \mathrm{E}+1 & 1.159 \\ 5.0 \mathrm{E}-9 & 6.080 \mathrm{E}+4 & 1.549 & 2.0 \mathrm{E}-3 & 3.294 \mathrm{E}+1 & 1.113 \\ 1.0 \mathrm{E}-8 & 9.927 \mathrm{E}+4 & 1.549 & 3.0 \mathrm{E}-3 & 2.172 \mathrm{E}+1 & 1.086 \\ 2.0 \mathrm{E}-8 & 1.323 \mathrm{E}+5 & 1.548 & 5.0 \mathrm{E}-3 & 1.360 \mathrm{E}+1 & 1.051 \\ 5.0 \mathrm{E}-8 & 9.936 \mathrm{E}+4 & 1.544 & 7.2 \mathrm{E}-3 & 1.009 \mathrm{E}+1 & 1.025 \\ 1.0 \mathrm{E}-7 & 7.168 \mathrm{E}+4 & 1.540 & 1.0 \mathrm{E}-2 & 7.455 & 1.000 \\ 2.0 \mathrm{E}-7 & 5.176 \mathrm{E}+4 & 1.534 & 2.0 \mathrm{E}-2 & 3.920 & 9.459 \mathrm{E}-1 \\ 3.0 \mathrm{E}-7 & 4.188 \mathrm{E}+4 & 1.529 & 3.0 \mathrm{E}-2 & 2.861 & 9.119 \mathrm{E}-1 \\ 5.0 \mathrm{E}-7 & 3.258 \mathrm{E}+4 & 1.522 & 5.0 \mathrm{E}-2 & 1.945 & 8.642 \mathrm{E}-1 \\ 7.2 \mathrm{E}-7 & 2.645 \mathrm{E}+4 & 1.515 & 7.2 \mathrm{E}-2 & 1.496 & 8.261 \mathrm{E}-1 \\ 1.0 \mathrm{E}-6 & 2.141 \mathrm{E}+4 & 1.508 & 1.0 \mathrm{E}-1 & 1.177 & 7.885 \mathrm{E}-1 \\ 2.0 \mathrm{E}-6 & 1.404 \mathrm{E}+4 & 1.491 & 2.0 \mathrm{E}-1 & 8.130 \mathrm{E}-1 & 6.908 \mathrm{E}-1 \\ 3.0 \mathrm{E}-6 & 1.026 \mathrm{E}+4 & 1.479 & 3.0 \mathrm{E}-1 & 6.234 \mathrm{E}-1 & 6.188 \mathrm{E}-1 \\ 5.0 \mathrm{E}-6 & 6.952 \mathrm{E}+3 & 1.462 & 5.0 \mathrm{E}-1 & 4.503 \mathrm{E}-1 & 5.117 \mathrm{E}-1 \\ 7.2 \mathrm{E}-6 & 5.137 \mathrm{E}+3 & 1.448 & 7.2 \mathrm{E}-1 & 3.414 \mathrm{E}-1 & 4.242 \mathrm{E}-1 \\ 1.0 \mathrm{E}-5 & 3.933 \mathrm{E}+3 & 1.436 & 1.0 & 2.648 \mathrm{E}-1 & 3.379 \mathrm{E}-1 \\ 2.0 \mathrm{E}-5 & 2.354 \mathrm{E}+3 & 1.405 & 1.5 & 1.896 \mathrm{E}-1 & 2.220 \mathrm{E}-1 \\ 3.0 \mathrm{E}-5 & 1.671 \mathrm{E}+3 & 1.385 & 2.0 & 1.247 \mathrm{E}-1 & 1.412 \mathrm{E}-1 \\ 5.0 \mathrm{E}-5 & 1.117 \mathrm{E}+3 & 1.357 & 3.0 & 4.536 \mathrm{E}-2 & 6.077 \mathrm{E}-2 \\ 7.2 \mathrm{E}-5 & 8.053 \mathrm{E}+2 & 1.336 & 4.0 & 1.906 \mathrm{E}-2 & 2.976 \mathrm{E}-2 \\ 1.0 \mathrm{E}-4 & 6.033 \mathrm{E}+2 & 1.317 & 5.0 & 1.053 \mathrm{E}-2 & 1.501 \mathrm{E}-2 \\ 2.0 \mathrm{E}-4 & 3.295 \mathrm{E}+2 & 1.272 & 6.0 & 5.060 \mathrm{E}-3 & 7.328 \mathrm{E}-3 \\ 3.0 \mathrm{E}-4 & 2.297 \mathrm{E}+2 & 1.244 & 8.0 & 1.268 \mathrm{E}-3 & 1.818 \mathrm{E}-3 \\ 5.0 \mathrm{E}-4 & 1.345 \mathrm{E}+2 & 1.208 & 10.0 & 3.000 \mathrm{E}-4 & 4.235 \mathrm{E}-4 \\ & & & & & \end{array}$

$\begin{array}{ll}\text { File \# ACRPBC14 Date } & 8-10-84 \quad \text { Reactor Shot \#2134 } \\ & =2.84 \\ \text { SD for } 9 \text { foils } & 16.45 \\ \text { SI } & =2.419 \mathrm{E}+13 \mathrm{n} /\left(\mathrm{cm}^{2} \cdot \mathrm{MJ}\right) \\ \text { Total Fluence per MJ } & 75.0 \mathrm{MJ} \\ \text { Total Energy in Core } & 1.814 \mathrm{E}+15 \mathrm{n} / \mathrm{cm}^{2} \\ \text { Total Neutrons } & 9.485 \mathrm{E}+11 \mathrm{n} /\left(\mathrm{cm}^{2} \cdot \mathrm{MJ}\right) \\ \text { Fluence }>3 \mathrm{MeV} \text { per MJ } & =\text { not measured }\end{array}$




\section{(12) ACRR Fuel-Ringed External Cavity (FREC) spectrum}

(FREC2344)

The ACRR Fuel-Ringed External Cavity (FREC) is shown in Figure V.21. The experimental cavity is $38 \mathrm{~cm}$ in diameter. A spectrum measurement was made $58.4 \mathrm{~cm}$ above the bottom of this cavity where some electronic circuits had been exposed. The location is shown in Figure V.22. The foil set was mounted within a $17-\mathrm{cm}$ diameter horizontal circle centered in the cavity as shown in Figure V.23. Additional nickel foils were placed among the rest to measure the uniformity of the fluence. The maximum spread in the nickel set occurred along a radial line away from the main core and amounted to $6 \%$. Corrections to the activities were made for this profile based on the individual foil location.

The reactor run was a pulse, so no corrections for activity decay during irradiation were necessary. The exposed set of foils is listed in Table V.23. Those foils not used in the adjustment procedure are marked with an asterisk. The reasons for their deletion from the analysis have been discussed above. This spectrum has such a very large fluence in the epithermal and thermal region that four reactions exhibited most of their response there: Au197(n,r)Au198 both inside and outside a cadmium cover, and Na23(n,r) Na24 both inside and outside a cadmium cover. We found that a trial function that did not provide a very close agreement in the calculated activities at the outset caused SANDII to try to fit the activities anywhere it could. Peaks occurred in the spectrum, at sodium resonances for example, if the trial spectrum was only a few percent low at around $1 \mathrm{ev}$. Although it took many attempts to find a compatible spectrum shape in low-energy regions, there was very little latitude in the result. In other words, the solution was very well determined for the given activities. However, any measurement errors had a significant effect on the low-energy spectrum shape. During the adjustment exercises, the various SIs did not vary by more than 0.1 out of 9. Thus, the calculated damage for silicon would not have varied significantly.

Table V.24 contains the spectral characteristics, and the spectrum shape is shown in Figure V.24. 


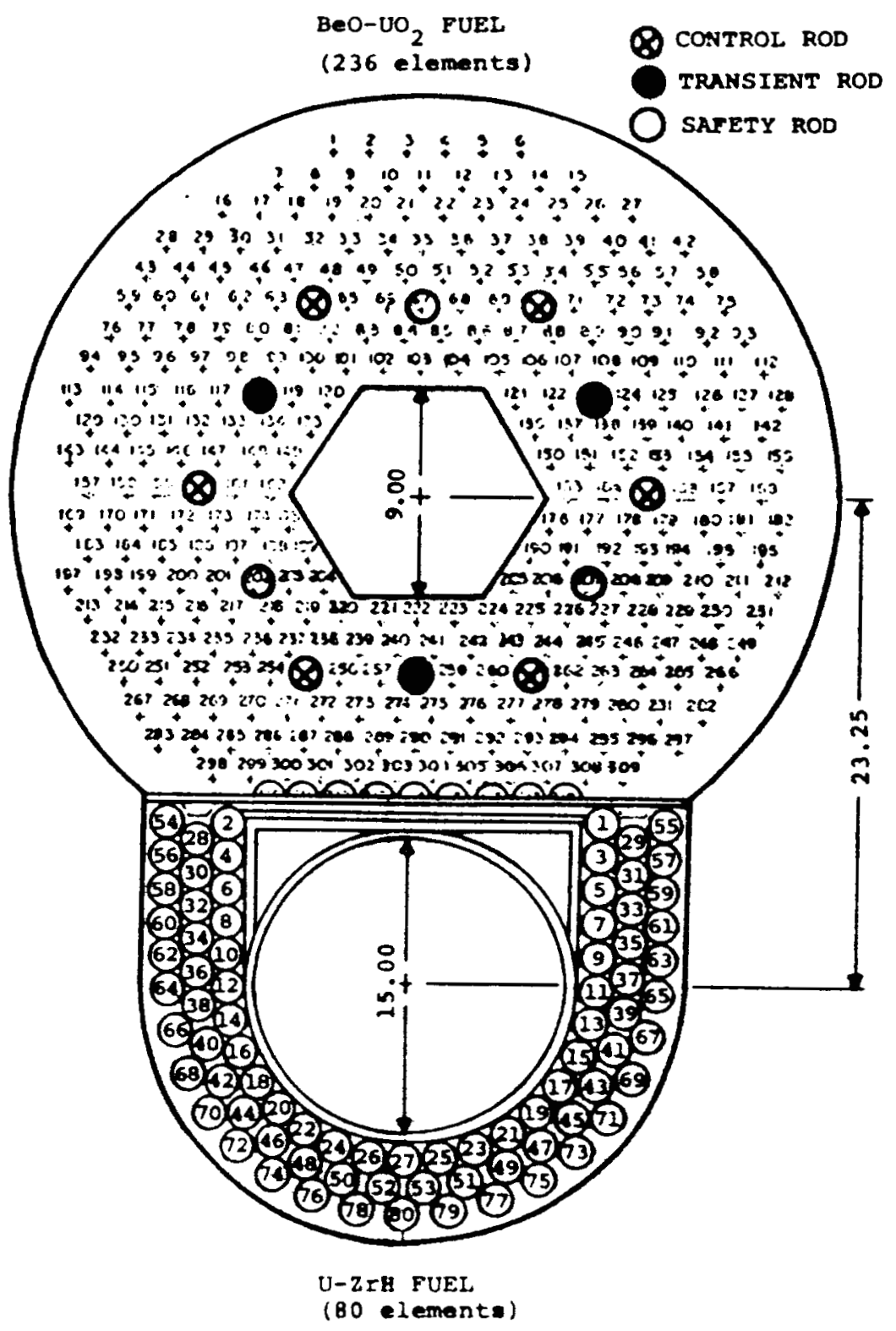

Figure V.21. ACRR Core Fuel Arrangement 
Note: Dimensions are in inches.

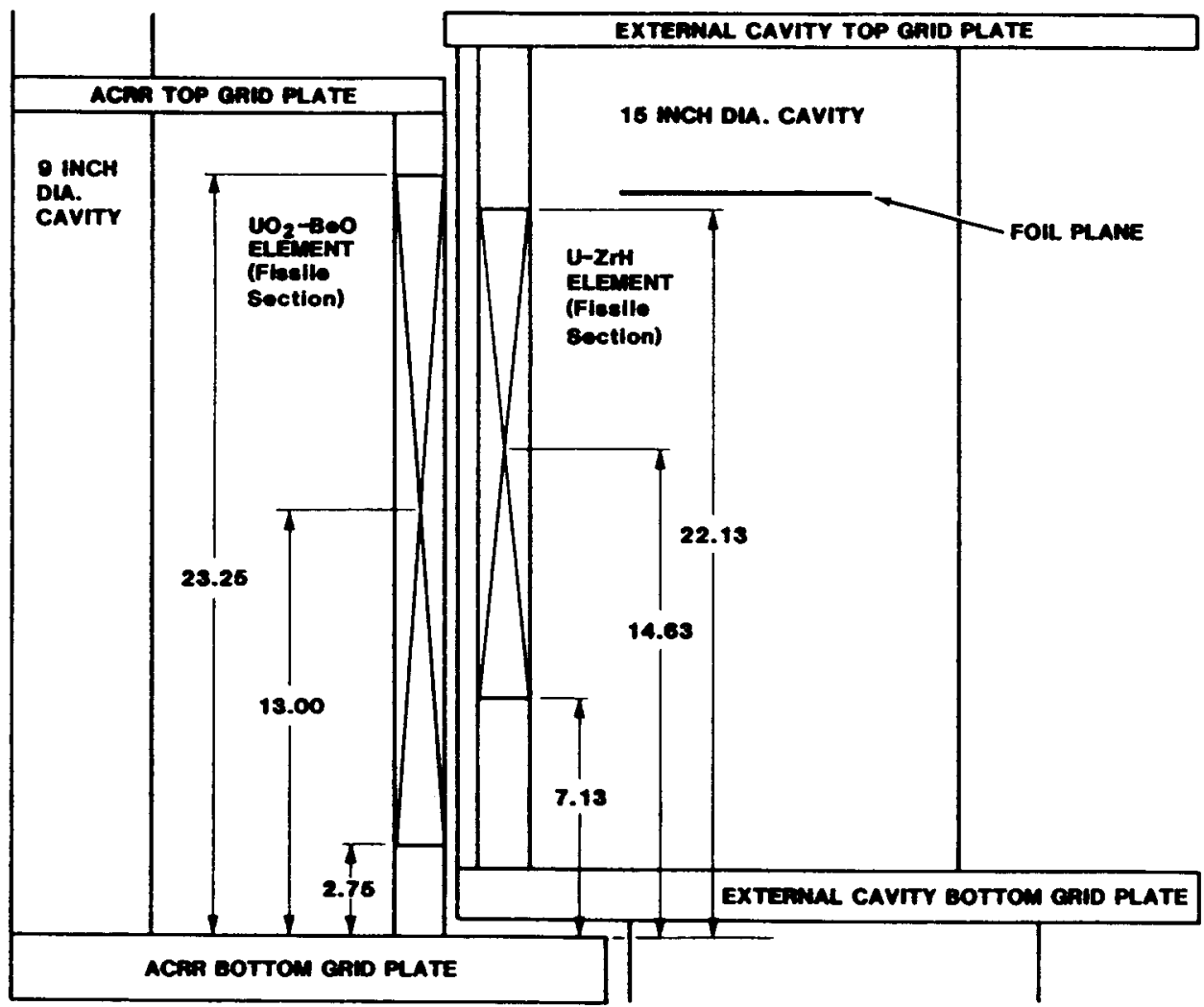

Figure V.22. Vertical Cross Section Through ACRR Core 


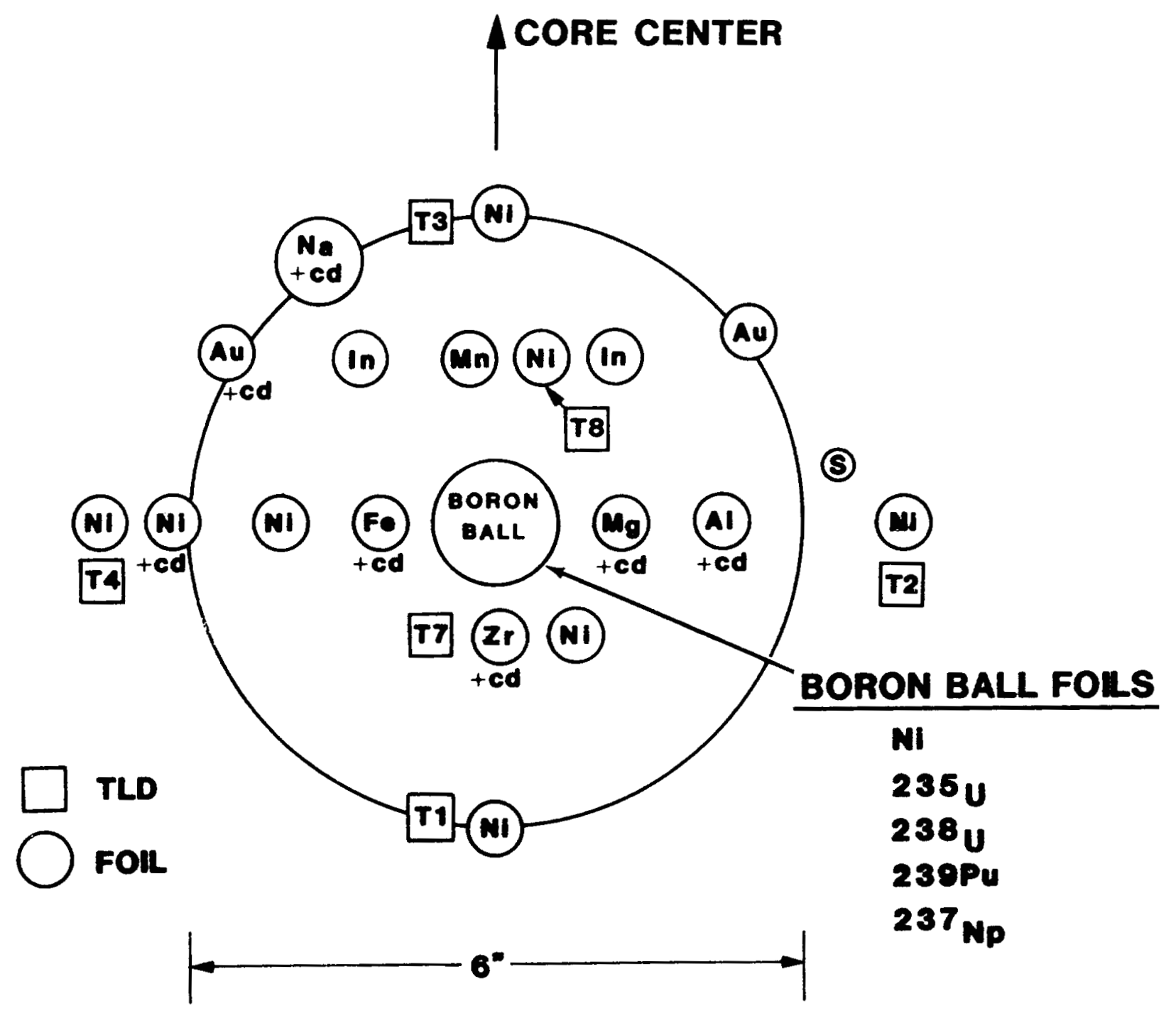

Figure v.23. Foil Arrangement in Fuel-Ringed External Cavity 
Table V.23

Foil Activities for ACRR Fuel-Ringed External Cavity

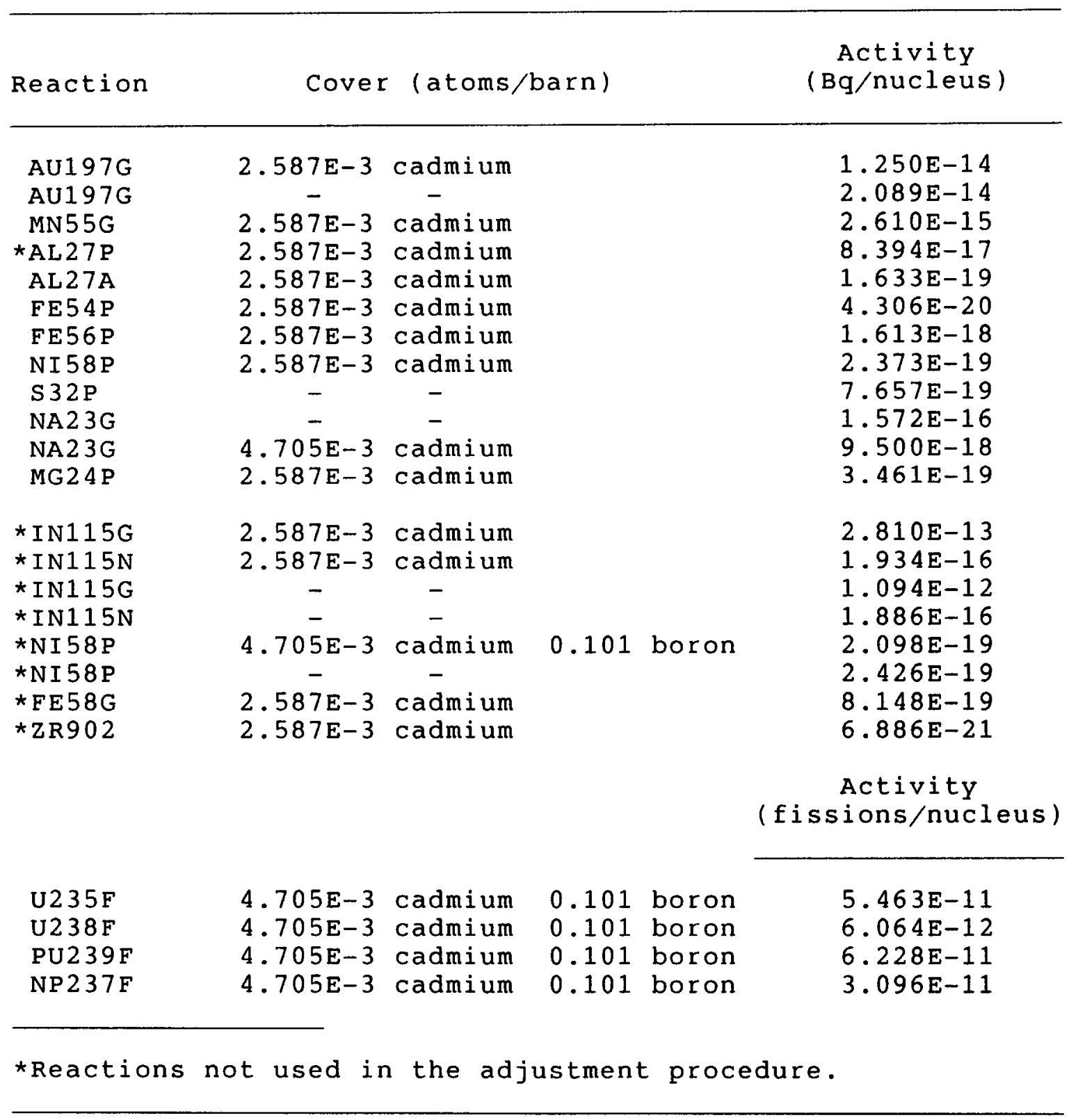


Table V. 24

ACRR Fuel-Ringed External Cavity, Free-Field spectrum

$\mathrm{E}(\mathrm{MeV})$ Differential Integral

$E(\mathrm{MeV})$ Differential Integral

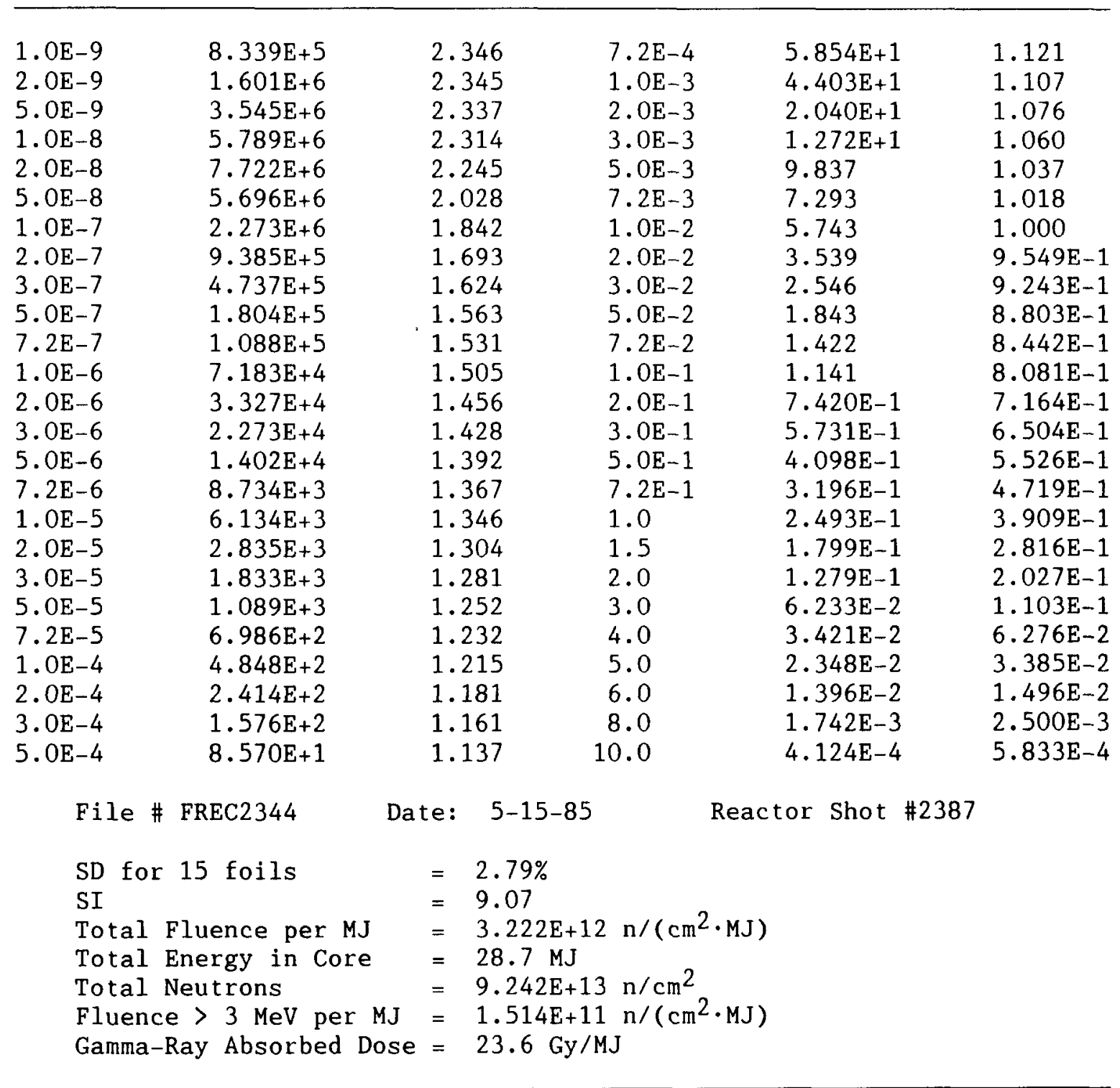




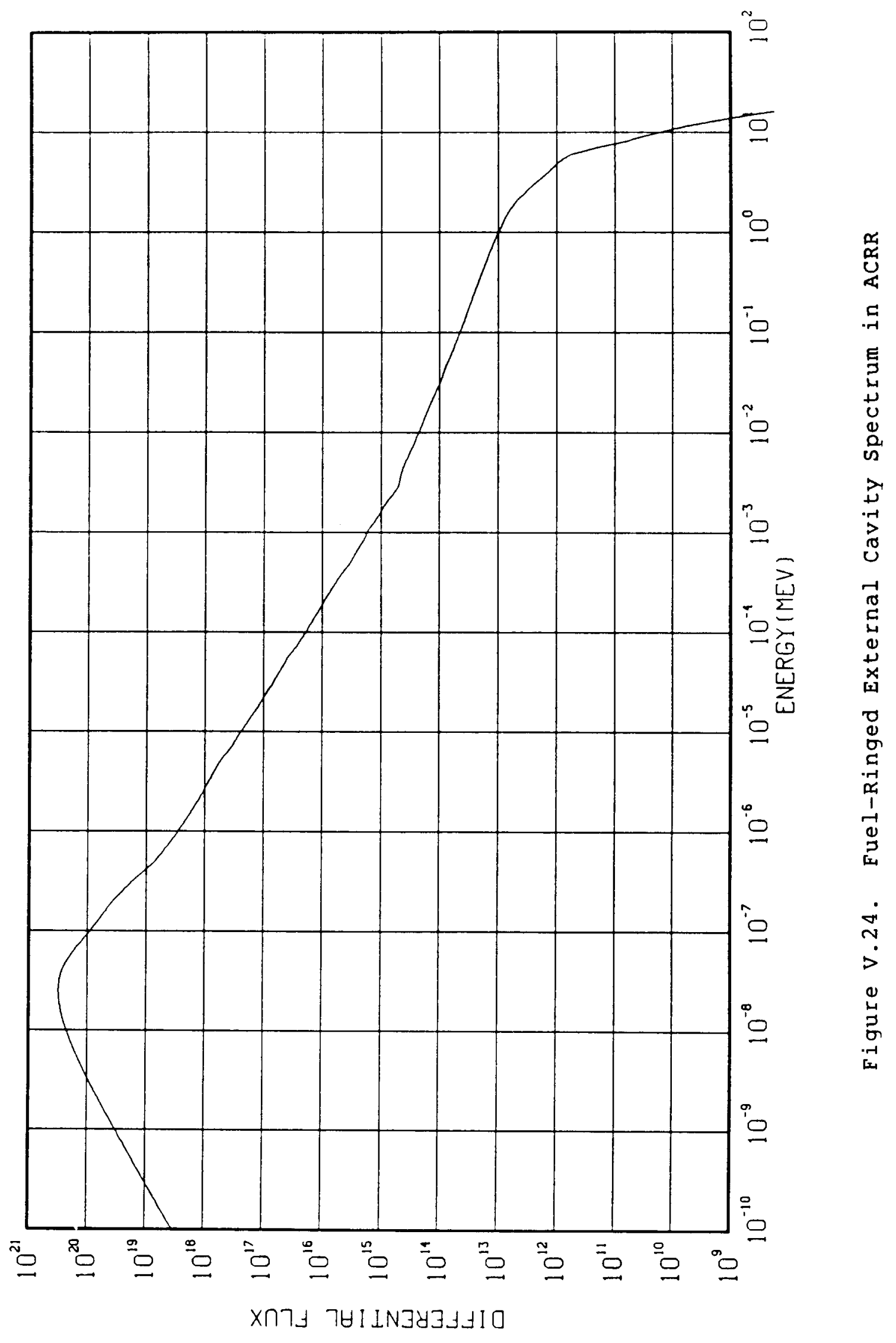




\section{(13) ACRR Spectrum in New Lead-Boron Cavity Liner (LBACRR12)}

A new ACRR cavity liner was recently tested. The purpose of the liner is to reduce gamma-ray and thermal neutron fluences as much as possible and still leave a $12.7-\mathrm{cm}$ diameter central cavity for experiments. The configuration is shown in Figure V.25. The cylindrical walls contain $3.8 \mathrm{~cm}$ of lead inside $1.27 \mathrm{~cm}$ of natural $\mathrm{B}_{4} \mathrm{C}$ powder that has been shaken down. The top and bottom end caps have $2.5 \mathrm{~cm}$ of lead inside $1 \mathrm{~cm}$ of $\mathrm{B}_{4} \mathrm{C}$. In this case, the $A C R R$ was operated at $100 \mathrm{~kW}$ for $500 \mathrm{~s}$, and good exposures were obtained on all of the foils. Once again, two sets of foils were run, one with the boron ball in place and one without. Three sulfur pellets were exposed to provide a more accurate sulfur activity.

The foil activities are listed in Table V.25. Those not used in the analysis are marked by asterisks. The spectrum is listed in Table V.26 and is shown in Figure V.26. Substantial lowering of the spectrum in the thermal region is evident, as compared to the free-field situation. In addition, the fast fluence has also been significantly reduced by the inelastic scattering of neutrons in the lead. This latter reduction raised the $\mathrm{SI}$ to 16.8 . The decrease in fluence below $10 \mathrm{keV}$ caused by the $\mathrm{B}_{4} \mathrm{C}$ layer does not affect the SI. The relative fluence in the vicinity of the gold resonance at $5 \mathrm{eV}$ was reduced by a factor of 6 with this liner, as compared to a factor of 2 with the older lead liner (Case 11).

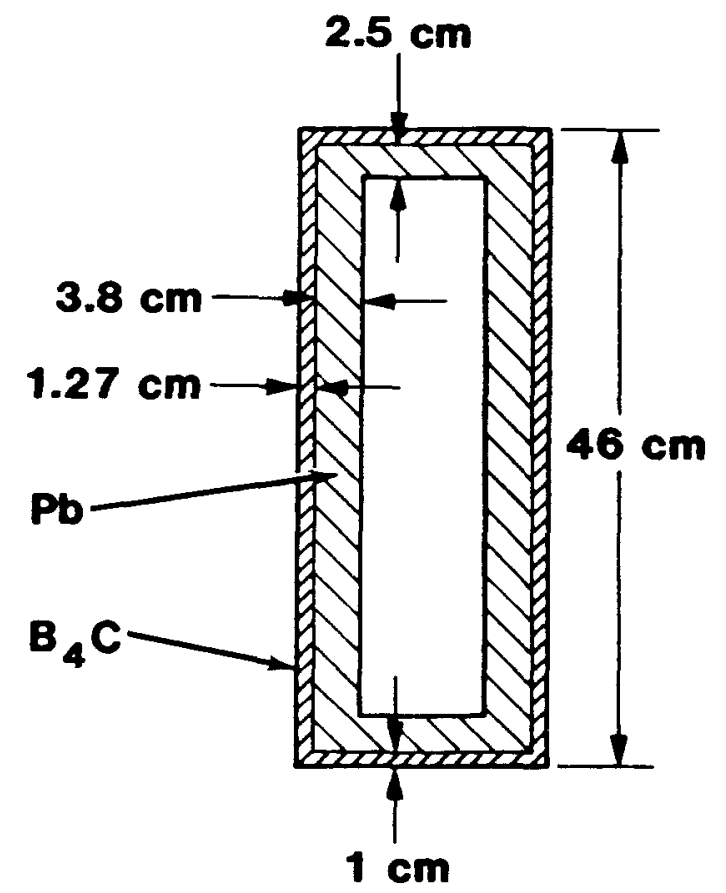

Figure V.25. Drawing of New Lead-Boron ACRR Cavity Liner 
Table V. 25

Foil Activities for New Lead-Boron Liner in ACRR Cavity

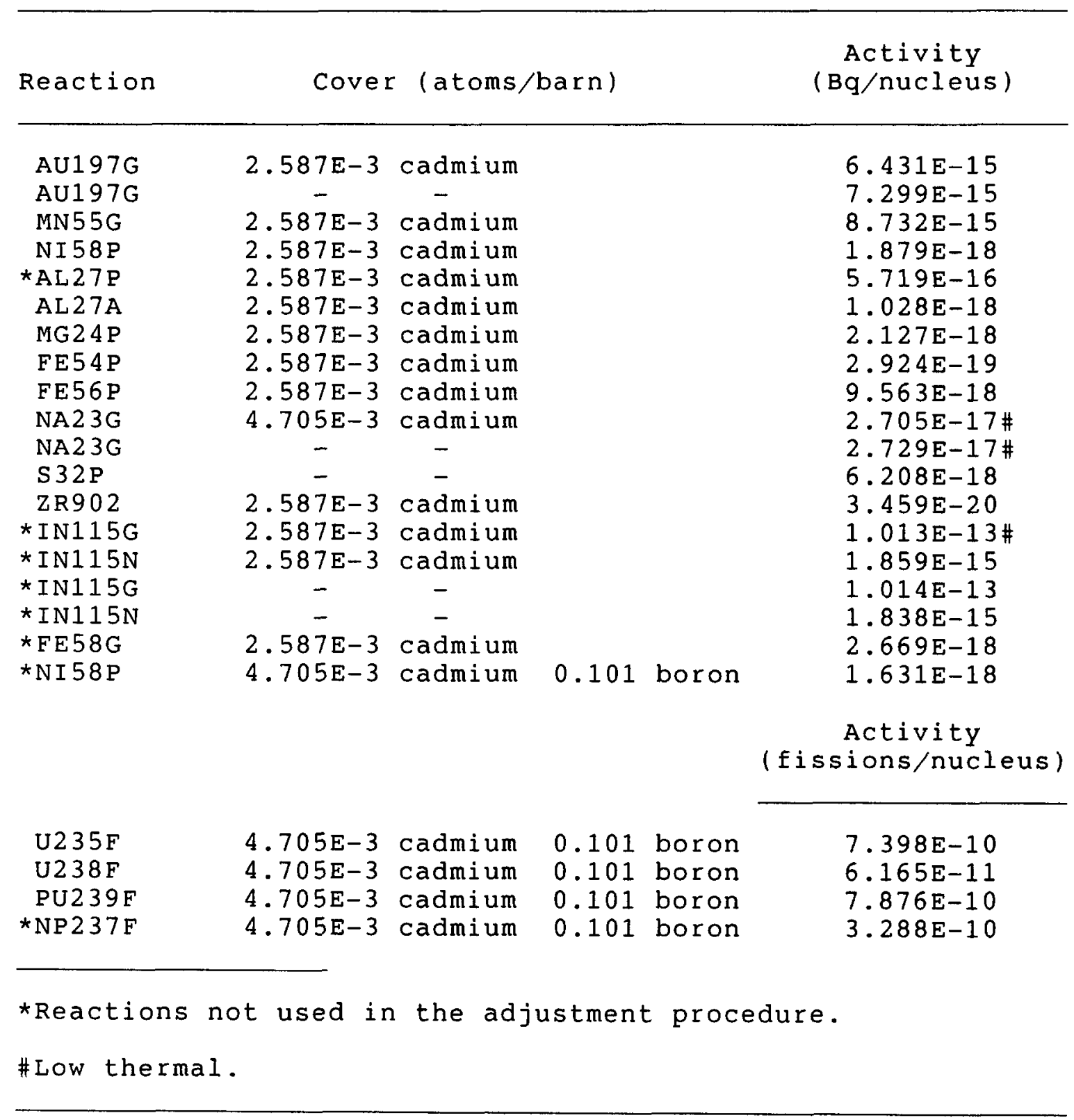


Table V.26

ACRR Lead-Boron-Lined Cavity spectrum

$($ normalized to $\Phi(E>10 \mathrm{keV})=1.00)$

\section{$E(\mathrm{MeV})$ Differential Integral $\mathrm{E}(\mathrm{MeV})$ Differential Integral}

\begin{tabular}{llllll}
\hline $1.0 \mathrm{E}-9$ & 1.466 & 1.166 & $7.2 \mathrm{E}-4$ & $3.930 \mathrm{E}+1$ & 1.106 \\
$2.0 \mathrm{E}-9$ & 2.933 & 1.166 & $1.0 \mathrm{E}-3$ & $3.031 \mathrm{E}+1$ & 1.096 \\
$5.0 \mathrm{E}-9$ & 7.332 & 1.166 & $2.0 \mathrm{E}-3$ & $1.752 \mathrm{E}+1$ & 1.073 \\
$1.0 \mathrm{E}-8$ & $1.466 \mathrm{E}+1$ & 1.166 & $3.0 \mathrm{E}-3$ & $1.229 \mathrm{E}+1$ & 1.058 \\
$2.0 \mathrm{E}-8$ & $2.933 \mathrm{E}+1$ & 1.166 & $5.0 \mathrm{E}-3$ & 9.093 & 1.037 \\
$5.0 \mathrm{E}-8$ & $7.331 \mathrm{E}+1$ & 1.166 & $7.2 \mathrm{E}-3$ & 7.252 & 1.019 \\
$1.0 \mathrm{E}-7$ & $1.355 \mathrm{E}+2$ & 1.166 & $1.0 \mathrm{E}-2$ & 5.999 & 1.000 \\
$2.0 \mathrm{E}-7$ & $2.280 \mathrm{E}+2$ & 1.166 & $2.0 \mathrm{E}-2$ & 4.059 & $9.508 \mathrm{E}-1$ \\
$3.0 \mathrm{E}-7$ & $2.803 \mathrm{E}+2$ & 1.166 & $3.0 \mathrm{E}-2$ & 3.172 & $9.140 \mathrm{E}-1$ \\
$5.0 \mathrm{E}-7$ & $3.452 \mathrm{E}+2$ & 1.166 & $5.0 \mathrm{E}-2$ & 2.349 & $8.586 \mathrm{E}-1$ \\
$7.2 \mathrm{E}-7$ & $3.882 \mathrm{E}+2$ & 1.166 & $7.2 \mathrm{E}-2$ & 1.964 & $8.108 \mathrm{E}-1$ \\
$1.0 \mathrm{E}-6$ & $4.182 \mathrm{E}+2$ & 1.166 & $1.0 \mathrm{E}-1$ & 1.629 & $7.601 \mathrm{E}-1$ \\
$2.0 \mathrm{E}-6$ & $4.468 \mathrm{E}+2$ & 1.166 & $2.0 \mathrm{E}-1$ & $9.782 \mathrm{E}-1$ & $6.342 \mathrm{E}-1$ \\
$3.0 \mathrm{E}-6$ & $4.404 \mathrm{E}+2$ & 1.165 & $3.0 \mathrm{E}-1$ & $6.611 \mathrm{E}-1$ & $5.521 \mathrm{E}-1$ \\
$5.0 \mathrm{E}-6$ & $4.010 \mathrm{E}+2$ & 1.164 & $5.0 \mathrm{E}-1$ & $4.012 \mathrm{E}-1$ & $4.471 \mathrm{E}-1$ \\
$7.2 \mathrm{E}-6$ & $3.797 \mathrm{E}+2$ & 1.163 & $7.2 \mathrm{E}-1$ & $2.805 \mathrm{E}-1$ & $3.719 \mathrm{E}-1$ \\
$1.0 \mathrm{E}-5$ & $3.421 \mathrm{E}+2$ & 1.162 & 1.0 & $2.077 \mathrm{E}-1$ & $3.028 \mathrm{E}-1$ \\
$2.0 \mathrm{E}-5$ & $2.599 \mathrm{E}+2$ & 1.159 & 1.5 & $1.617 \mathrm{E}-1$ & $2.090 \mathrm{E}-1$ \\
$3.0 \mathrm{E}-5$ & $2.177 \mathrm{E}+2$ & 1.157 & 2.0 & $1.157 \mathrm{E}-1$ & $1.373 \mathrm{E}-1$ \\
$5.0 \mathrm{E}-5$ & $1.757 \mathrm{E}+2$ & 1.153 & 3.0 & $4.670 \mathrm{E}-2$ & $5.939 \mathrm{E}-2$ \\
$7.2 \mathrm{E}-5$ & $1.417 \mathrm{E}+2$ & 1.150 & 4.0 & $1.849 \mathrm{E}-2$ & $2.821 \mathrm{E}-2$ \\
$1.0 \mathrm{E}-4$ & $1.181 \mathrm{E}+2$ & 1.146 & 5.0 & $9.796 \mathrm{E}-3$ & $1.426 \mathrm{E}-2$ \\
$2.0 \mathrm{E}-4$ & $8.803 \mathrm{E}+1$ & 1.136 & 6.0 & $5.782 \mathrm{E}-3$ & $6.448 \mathrm{E}-3$ \\
$3.0 \mathrm{E}-4$ & $7.332 \mathrm{E}+1$ & 1.128 & 8.0 & $8.432 \mathrm{E}-4$ & $1.227 \mathrm{E}-3$ \\
$5.0 \mathrm{E}-4$ & $5.017 \mathrm{E}+1$ & 1.116 & 10.0 & $2.014 \mathrm{E}-4$ & $2.953 \mathrm{E}-4$
\end{tabular}

$\begin{array}{ll}\text { File \# LBACRR12 Date: } & 11-4-85 \quad \text { Reactor Shot \#2607 } \\ & =3.5 \% \\ \text { SD for } 16 \text { foils } & =16.84 \\ \text { SI } & 1.233 \mathrm{E}+13 \mathrm{n} /\left(\mathrm{cm}^{2} \cdot \mathrm{MJ}\right) \\ \text { Total Fluence per MJ } & 50.0 \mathrm{MJ} \\ \text { Total Energy in Core } & 6.166 \mathrm{E}+14 \mathrm{n} / \mathrm{cm}^{2} \\ \text { Total Neutrons } & 6.280 \mathrm{E}+11 \mathrm{n} /\left(\mathrm{cm}^{2} \cdot \mathrm{MJ}\right) \\ \text { Fluence }>3 \mathrm{MeV} \text { per MJ } & =12.8 \mathrm{~Gy} / \mathrm{MJ}\end{array}$




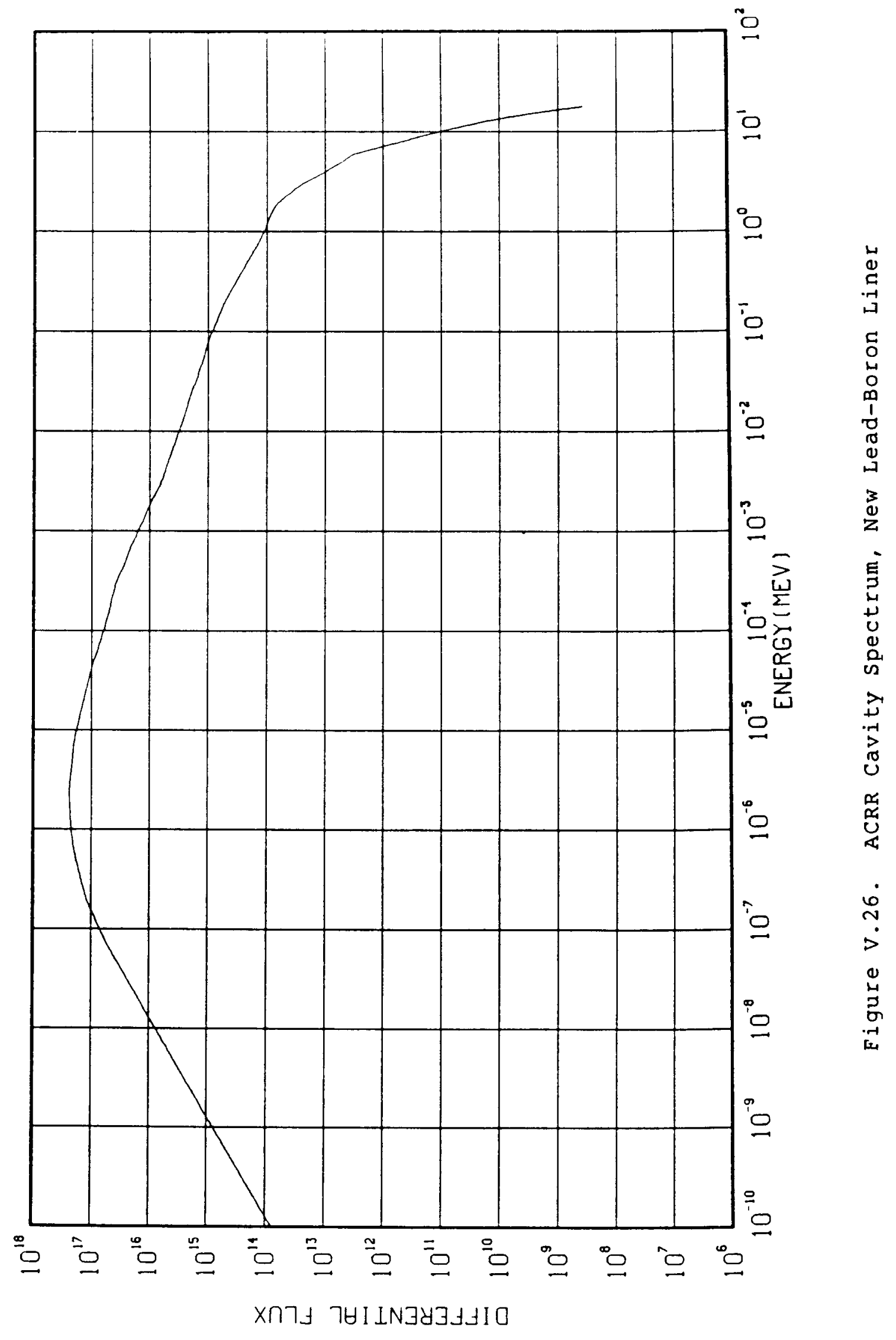




\section{B. Goodness of Fit for the Activities}

As the list of measured spectra began to grow, it became obvious that for some foil reactions there was a tendency for the measured activity to remain higher or lower than the calculated values. In some cases, such a tendency was consistent over the whole set of measurements; in others, the consistency was limited to a subset of spectra that were related in some way. It has been pointed out above, for example, that the activity resulting from the In115(n, n') In115 reaction is consistently high in all the SANDII-adjusted spectra that have been measured. It was clear that something could be learned about the foil sets, the cross sections for each reaction, the counting techniques, and the environments, by examining the trends in the deviations.

Table V.27 lists the percentage deviation of the measured from the calculated activity for each reaction used in the adjustment, for each of the 13 spectra that have been obtained. A positive value indicates that the measured activity is higher for the reaction than that calculated with this final spectrum. To obtain better agreement, the spectrum would have to be raised somewhere in the energy region where this particular reaction is sensitive. (This action might lead to poorer agreement with other activities in the set.) Those activities not used in the adjustment process are underlined.

We now examine each reaction and point out some correlations. The discussions below include a few reactions that do not appear in Table V.27 and that were not used in the adjustments.

(1) Gold, bare and cadmium-covered - It is not surprising that excellent agreements were normally obtained on the gold activities, because these foils usually responded in energy regions where they did not conflict with others. SANDII solutions could, therefore, easily fit the data. The deviations appear to be random, associated only with normal experimental error.

(2) IN115G, bare and cadmium-covered (not in Table V.27) - The measured activities are far lower than calculated because of self-shielding. This explanation was proven by placing the primary foil between indium sheets. The activity was then severely depressed. Although self-shielding might be corrected for, we plan to acquire either very thin or very dilute foils for future foil sets. Successful incorporation of this reaction would confirm spectrum shapes in the thermal region. 


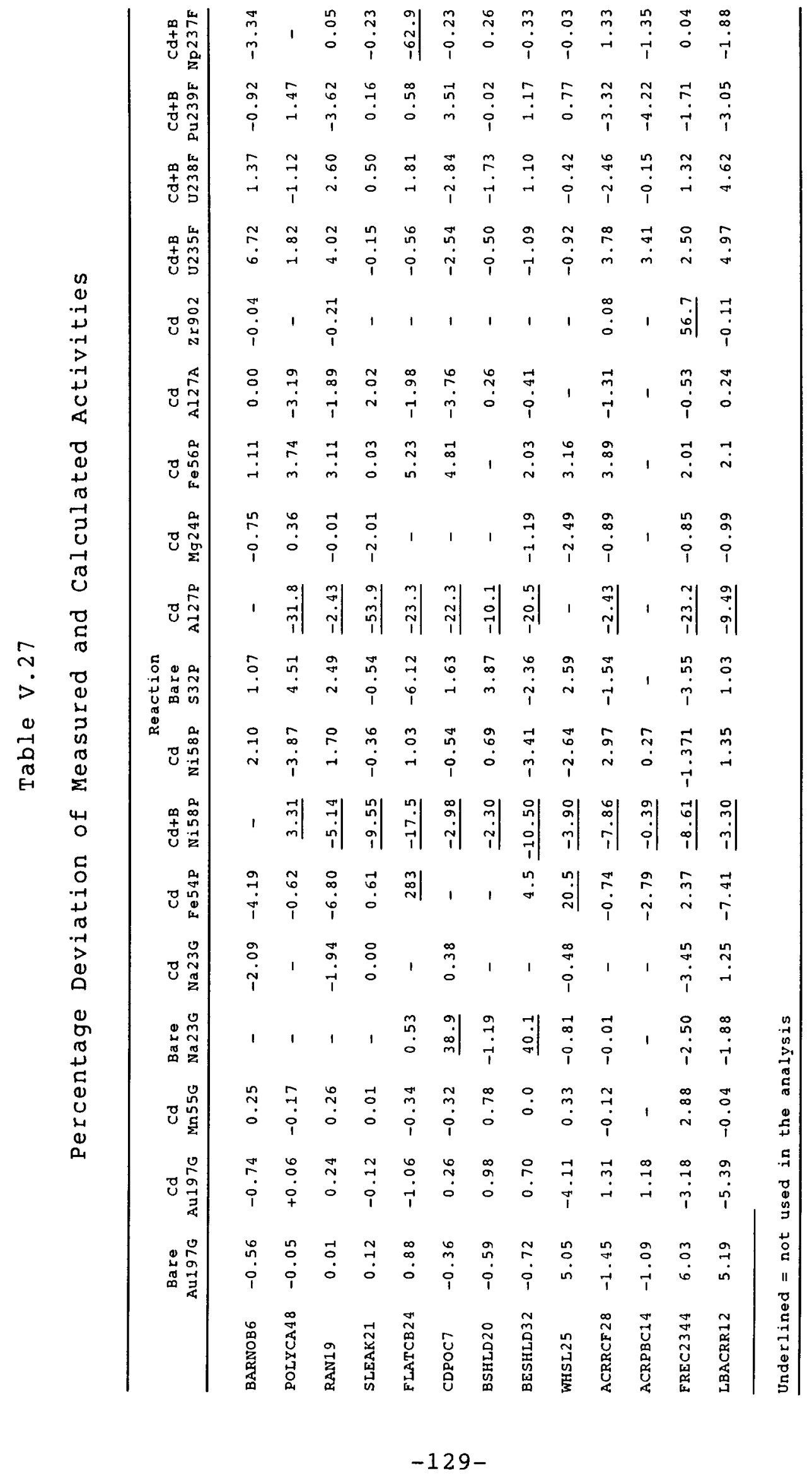


(3) IN115N, bare and cadmium-covered (not in Table V.27) - This reaction has a threshold at about 1 MeV. For all spectra, the measured activity is higher than the calculated one. One explanation suggested by personnel from WSMR was that the reaction is also excited by $r$ rays. [26] In support of this explanation, there is a tendency for the indium activity induced inside the cadmium cover to exceed that outside the cover when the thermal neutron fluence is high. When cadmium absorbs thermal neutrons, many high-energy gamma rays are emitted. On average, the bare IN $115 \mathrm{~N}$ reaction (no cadmium cover) was $9.4 \% \mathrm{high}$, and the cadmium-covered ones were $13.6 \% \mathrm{high}$.

(4) MN55G, cadmium-covered - No recognizable pattern has been observed.

(5) NA23G, bare and cadmium-covered - In some of the later experiments, a sodium chloride pellet inside a cadmium cover was included in the set so as to obtain a more restricted response at the higher energies. we have found that this addition provides improved spectrum shape discrimination both at thermal and at 3-kev energies. No deviation pattern based on foil sets has been apparent in these activities. Two of the activities are far from agreement with the rest. At this time the disagreements are ascribed to experimental or counting error.

(6) FE54P, cadmium-covered - No obvious pattern. In the file WHSL25, generated from the incomplete foil set exposed at the WSMR FBR, this reaction could not be made compatible with the rest, so it was left out of the adjustment procedure. The FE54P reaction activity was counted 2 months after the rest of the foil set in WHSL 25 .

(7) NI58P, cadmium-covered, with and without boron - The nickel outside the boron exhibits no recognizable deviation pattern, but the nickel in the boron ball is consistently lower, as has been noted earlier in the text. (See Appendix $C$ also.) We believe that SANDII inadequately corrects for the attenuation of the neutron fluence in the ball. The experimental and the calculated attenuations are considerably larger than those calculated by the code with purely exponential attenuation. [27]

(8) S32P - The deviations of the sulfur measurements from the calculated values seem random. There is generally good agreement with the nickel foils. 
(9) AL27P, cadmium-covered (not included in Table V.27) The activities measured in this case are consistently low and very erratic. This foil has a decay time of only 9 minutes, so the timing of the irradiation and the counting is extremely important. We have tried to be careful in this regard, but perhaps the correction factors are incorrect or the timing is erroneous. This reaction is not used in the adjustment process. A mystery not yet solved is that the disagreement is much less for free-field cavity measurements.

(10) MG24P, cadmium-covered - This reaction fits very well with all the spectra for which sufficient counts were recorded during the analysis. Poor statistics are obtained fairly frequently from this foil because the threshold is high $(\sim 6 \mathrm{MeV})$, the decay time of ${ }^{24} \mathrm{Na}$ is fairly short, the spectrum drops off rapidly with energy, and the cross section is low.

(11) FE56P, cadmium-covered - This reaction activity consistently averages $3.7 \%$ high in all the spectra obtained from fast-burst reactors. The agreement is $2.1 \%$ at the ACRR. This difference by reactor type suggests that we are not seeing a problem with the $56 \mathrm{Fe}$ foil cross section only. The difference would then be caused by a spectrum-shape difference between the two reactor types.

Another explanation has been investigated. The daughter product of the $\operatorname{Fe} 56(\mathrm{n}, \mathrm{p}) \mathrm{Mn} 56$ reaction is also the daughter of the $\operatorname{Mn} 55(\mathrm{n}, \gamma) \operatorname{Mn} 56$ reaction. Even a small amount of contamination of $55 \mathrm{Mn}$ in the $55 \mathrm{Fe}$ foil would cause a large change in the activity that produces the indistinguishable gamma-ray lines. This is because the $55 \mathrm{Mn}$ reaction has a much larger spectrum-averaged cross section. In order to demonstrate that contamination was not the cause of this discrepancy, iron and manganese foils were exposed in cadmium covers at SPR III. We immediately read the foils, the cadmium covers, and the bare detector system (after the foils and covers had been counted there) and found no contamination of $55_{\mathrm{Mn}}$ anywhere. The evidence indicates that contamination is not the explanation for the high ${ }^{56} \mathrm{Fe}$ readings in comparison with the other foils.

(12) AL27A, cadmium-covered - The FE56P reaction has a threshold near that of $A L 27 \mathrm{~A}$, so if iron tends to be high, it is natural for AL27A to be low (or vice versa), because SANDII tries to fit both activities. 
(13) ZR902, cadmium-covered - The threshold for this reaction is so high $(14 \mathrm{MeV})$ that in many cases there is not enough fluence to produce enough activity for the measurements.

(14) U235F and PU239F in the boron ball - These two reactions are discussed together because they have similar, fairly flat responses above the $10-\mathrm{keV}$ threshold imposed by the boron. We see a complementary pattern here: For the SPR III cavity and the ACRR results, the $U 235 \mathrm{~F}$ is high and the PU239F is low. For the external fast-burst reactor spectra, the U235F is low and the PU239F is high. This suggests that slight differences in spectral shapes should be applied uniformly to the two categories in these results.

(15) U238F in the boron ball - No deviation pattern is evident.

(16) NP237F in the boron ball - The deviations achieved with this foil are very small except for the one obtained between cadmium-polyethylene blocks beside SPR III. 
C. Spectrum-Averaged Cross Sections, Activity, and Fluence Determinations

Many users of the SNLA radiation facilities prefer not to expose complete foil sets in order to determine a spectrum for their particular experiment. In fact, there may not be room or time to do this. However, if the spectrum in their configuration is already known from either measurement or calculation, the required information may be provided by a standardized measurement of integral fluence by sulfur or nickel foils. Sulfur, for example, provides a measure of the total fluence above its effective threshold of $3 \mathrm{MeV}$. The SI $[\Phi(E>10 \mathrm{keV}) / \Phi(\mathrm{E}>3 \mathrm{MeV})]$, when multiplied by the fluence obtained from sulfur, provides the fluence above $10 \mathrm{keV}$ (see Chapter III, section A). This 10-keV fluence is important when the displacement damage in silicon devices is being sought, because little displacement damage occurs below that energy. The displacement damage is

$$
\begin{aligned}
D & =\int_{10 \mathrm{keV}}^{\infty} \phi(E) \mathrm{d}(E) \mathrm{d} E \equiv \Phi(E>10 \mathrm{keV}) \overline{\mathrm{d}} \\
& =\Phi(E>3 \mathrm{MeV}) \cdot \mathrm{SI} \cdot \overline{\mathrm{d}}
\end{aligned}
$$

where

$$
\begin{aligned}
\bar{d} & =\text { spectrum-averaged damage } \\
d(E) & =\text { silicon displacement damage function or kerma } \\
\phi(E) & =\text { differential fluence. }
\end{aligned}
$$

Since the SIs for the spectra we have measured have already been determined, the next task is to determine the $\Phi$ ( $E>3 \mathrm{MeV})$ from the measured activity of sulfur pellets. This can be done with the use of the spectrum-averaged cross section, $\bar{\sigma}$, that connects the measured activity with $\Phi(\mathrm{E}>3 \mathrm{MeV})$.

$$
\bar{\sigma}(\text { thresh })=\frac{\int_{0}^{\infty} \phi(E) \sigma(E) d E}{\int_{\text {thresh }}^{\infty} \phi(E) d E} .
$$


If the activity, $A$, is determined simply by exponential decay of the reaction products, then

$$
\begin{aligned}
A & =\left.\frac{-d}{d t}\left[N e^{-\lambda t} \int_{0}^{\infty} \phi(E) \sigma(E) d E\right]\right|_{t=0} \\
& =\lambda N \int_{0}^{\infty} \phi(E) \sigma(E) d E
\end{aligned}
$$

where

$$
\begin{aligned}
& \lambda=\text { decay constant for reaction product } \\
& \mathrm{N}=\text { number of target nuclei in foil. }
\end{aligned}
$$

Also, one defines

$$
\Phi(\text { thresh }) \equiv \int_{\text {thresh }}^{\infty} \phi(E) d E
$$

If the threshold $=3 \mathrm{MeV}$, then

$$
\bar{\sigma}(E>3 \mathrm{MeV})=\frac{\mathrm{A}}{\mathrm{N} \lambda \Phi(\mathrm{E}>3 \mathrm{MeV})}
$$

or

$$
\Phi(E>3 \mathrm{MeV})=\frac{\mathrm{A}}{\mathrm{N} \lambda \overline{\boldsymbol{\sigma}}(E>3 \mathrm{MeV})} .
$$

In practice, the analyst can use the SANDII printout of the total fluence greater than $3 \mathrm{MeV}$ in determining $\bar{\sigma}$. For example, in the SPR III cavity experiment one obtains from the SANDII result $\Phi(E>3 \mathrm{MeV})=3.588 \mathrm{E}+13 \mathrm{n} / \mathrm{cm}^{2}$. Using the activity run option of SANDII, in which the code calculates the specific activity of each foil for a given spectrum, one finds that $\mathrm{A} / \mathrm{N}$ is $6.607 \mathrm{E}-18 \mathrm{~Bq} /$ nucleus. The calculated activity will be more reliable than the individual measured value because the former is derived from the full activity set. The decay constant $\lambda=5.614 \mathrm{E}-7 \mathrm{~s}^{-1}$ for sulfur. Therefore, the spectrum-averaged cross section from the sulfur pellet 
activity is

$$
\begin{aligned}
\bar{\sigma}_{\mathrm{s}} & =\frac{6.607 \times 10^{-18} \mathrm{~Bq} / \mathrm{nucleus}}{\left(5.614 \times 10^{-7} \mathrm{~s}^{-1}\right)\left(3.588 \times 10^{13} \mathrm{n} / \mathrm{cm}^{2}\right)} \\
\bar{\sigma}_{\mathrm{s}}(\text { cavity }) & =328 \mathrm{mb} \text { for SPR III central cavity. }
\end{aligned}
$$

If a facility user wishes to expose components in the $S P R$ III free-field central cavity and needs to know $\Phi(E>10 \mathrm{keV})$, he/she then calculates this in the following way:

$$
\Phi(\mathrm{E}>10 \mathrm{keV})=\Phi(\mathrm{E}>3 \mathrm{MeV}) \cdot \mathrm{SI} .
$$

Normally, the Radiation Dosimetry Laboratory will supply the user with a specific activity and a $\Phi(\mathrm{E}>3 \mathrm{MeV})$ for the sulfur pellet in the dosimetry printout. We found that the fluence printed by SANDII with the new cross-section set was conspicuously lower than that provided by the Radiation Dosimetry Laboratory. The spectrum-averaged cross section, $\bar{\sigma}(E>3 \mathrm{MeV})$, used by the laboratory was $285 \mathrm{mb}$ for the SPR III cavity, based on an older cross-section set.[28] (The older SANDII cross-section library also yielded $285 \mathrm{mb}$.)

We now drop the subscript s from $\bar{\sigma}$, because the following discussion is concerned only with sulfur. To verify that the new cross-section set provides better values for the $\bar{\sigma}$, the $252 \mathrm{Cf}$ neutron spectrum was integrated against the new sulfur cross section.[29] In this case, the total spectrum-averaged cross section was found to be

$$
\bar{\sigma}(\text { total })=\frac{\int_{0}^{\infty} \sigma(E) \phi_{C f}(E) d E}{\int_{0}^{\infty} \phi_{C f}(E) d E}=76 \mathrm{mb} .
$$

This is in agreement with the ASTM-recommended value.[30] The older cross-section set provided $67.6 \mathrm{mb}$. For the $\bar{\sigma}(\mathrm{E}>3 \mathrm{MeV})$, the new set yielded $\bar{\sigma}_{\mathrm{Cf}}(\mathrm{E}>3 \mathrm{MeV})=321 \mathrm{mb}$, and the old set yielded $\bar{\sigma}_{\mathrm{Cf}}(\mathrm{E}>3 \mathrm{MeV})=285 \mathrm{mb}$. (The subscript now identifies the spectrum from which the $\bar{\sigma}$ was derived.) 
However, this may not be the only source of error in the determination of the fluences, activities, spectrum-averaged cross sections, and damage functions. The determination of the activity of a sulfur pellet (Equation V.5) depends upon calibration. The discussion that follows examines the contributory factors in the determination of the parameters just mentioned and ties together foil-set measurements with sulfur dosimetry.

The relationship between the count rate recorded in the beta counter of the sulfur-pellet reader and the fluence that generated the activity in the pellet can be represented by the following formula:

$$
\Phi=k R
$$

where

$$
\begin{aligned}
& \Phi=\text { integral fluence that can contribute to induced } \\
& \mathrm{k}=\text { counter constant } \\
& R=\text { count rate corrected back to end of irradiation. }
\end{aligned}
$$

Note: The counter constant is not explicitly used by the Radiation Dosimetry Laboratory.

Generally, because sulfur has a reaction energy threshold at about $3 \mathrm{MeV}$, the fluence, $\Phi$, is taken to be $\Phi(E>3 \mathrm{MeV})$ by definition. We calibrate the counting system by exposing a large number of pellets to a fluence that has a known spectral shape and magnitude--a $252 \mathrm{Cf}$ spontaneous fission source, such as is available at the National Bureau of standards (NBS).[31] Pellets are exposed at such a calibration facility and then counted in the SNLA Radiation Dosimetry Laboratory. Thus, the counter constant for a californium source can be determined:

$$
{ }_{C f}=k_{C f}{ }_{C f}
$$

In the discussions which immediately follow, $\Phi(\mathrm{E}>3 \mathrm{MeV}) \equiv \Phi$. The fluence from any other exposure to a 252 Cf spectrum can be determined directly with the calibrated counting system since $\mathrm{k}_{\mathrm{C} f}$ has been determined. The counter constant will be different, however, for peliets exposed to a different spectral environment.

$$
\Phi_{r}=k_{r} R_{r}
$$


The $k_{r}$ can only be determined from a knowledge of the differential spectrum and the sulfur spectrum-averaged cross sections. At the outset, one starts with the fact that all pellets are read in the same counting system. Therefore, the counting efficiency, $\varepsilon$, is the same no matter what neutron radiation field produced the activity:

$$
\begin{aligned}
& R_{r}=\varepsilon A_{r} \\
& R_{C f}=\varepsilon A_{C f} .
\end{aligned}
$$

We return to the spectrum-averaged cross section:

$$
\bar{\sigma}(E>3 \mathrm{MeV}) \equiv \frac{\int_{0}^{\infty} \phi(E) \sigma(E) \mathrm{dE}}{\int_{3 \mathrm{MeV}}^{\infty} \phi(E) \mathrm{dE}}=\frac{\int_{0}^{\infty} \phi(E) \sigma(E) \mathrm{dE}}{\dot{\phi}(\mathrm{E}>3 \mathrm{MeV})} \quad(\mathrm{V} .14)
$$

Then

$$
A=N \lambda \int_{0}^{\infty} \phi(E) \sigma(E) d E \equiv N \lambda \Phi \bar{\sigma} .
$$

Hence

$$
\mathrm{k}=\frac{\mathrm{A}}{\mathrm{N} \lambda \overline{\bar{\sigma}} \mathrm{R}}
$$

Equations $V .12$ and $V .13$ show that the ratio of activity to detector count rate is a constant, independent of the exposure environment.

Therefore,

$$
\frac{{ }_{r} r}{R_{r}}=\frac{{ }_{C f}^{A}}{R_{C f}} .
$$


We substitute into the $k_{R}$ formula,

$$
k_{R}=\frac{A_{r}}{N \lambda \bar{\sigma}_{r}{ }_{r}}=\frac{{ }_{C f}}{N \lambda \bar{\sigma}_{r}{ }_{C f}} \text {. }
$$

Then

$$
k_{r}=\left(\frac{\bar{\sigma}_{C f}}{\bar{\sigma}_{r}}\right) k_{C f}
$$

and

$$
\Phi_{r}=\left(\frac{\bar{\sigma}_{C f}}{\bar{\sigma}_{r}}\right) k_{C f} R_{r} .
$$

One sees here explicitly the dependence of the measured fluence on the spectra in the two environments through the respective spectrum-averaged cross sections.

An interesting point can be made about the ratio $\bar{\sigma}_{C f} / \bar{\sigma}_{r}$ : If one uses a different cross-section set for sulfur to calculate the $\bar{\sigma}^{\prime} s$, this ratio would be little changed, because both spectrum-averaged cross sections would be expected to change by about the same amount if the spectral shapes are at all similar. In other words, $\Phi$ depends very strongly on the calibration factor $k$ but very weakly on the sulfur crosssection set used to calculate the two $\bar{\sigma}^{\prime} s$, as long as the same sulfur cross-section set is used for the calculation of $\bar{\sigma}_{C f}$ and of $\bar{\sigma}_{r}$.

The activity behaves differently. It can be determined without any knowledge of the spectrum that generated it (provided the calibration source spectrum is known)--as it must be because the measurement of the activity cannot depend on how it was generated.

$$
A_{r}=N \lambda \bar{\sigma}_{r} \Phi_{r}=N \lambda \bar{\sigma}_{r} k_{C f}\left(\frac{\bar{\sigma}_{C f}}{\bar{\sigma}_{r}}\right) R_{r}=N \lambda k_{C f} \bar{\sigma}_{C f}{ }^{R} .
$$


The formula (V.21) exhibits no dependence on the spectrum that generated this activity. In this case, the activity that is determined depends very strongly on the sulfur crosssection set used (through $\bar{\sigma}_{C f}$ ). We see that the calibration procedure and the cross-section sets have very different influences on the activity and the fluence determinations.

since the activity is needed in order to determine the spectrum, one needs to know how past determinations of the activity should be corrected. Define $\bar{\sigma}^{\circ}$ and $\bar{\sigma}^{n}$ to be the spectrum-averaged cross sections calculated with the old and new cross sections, respectively. Likewise, define $\mathrm{k}^{\mathrm{o}}$ and $\mathrm{k}^{\mathrm{n}}$ to be the counter constants determined by old and new $252 \mathrm{Cf}$ calibrations, respectively. Keep in mind that these are entirely independent factors.

$$
A_{r}^{n}=A_{r}^{o}\left(\frac{\sigma_{C f}^{n}}{\sigma_{C f}^{o}}\right)\left(\frac{k_{C f}^{n}}{\sigma_{C f}^{o}}\right) \text {. }
$$

Notice that the correction to the activity, Equation V.22, is independent of the neutron spectrum to which the sulfur is exposed and would be applied to all sulfur activities. The correction to the fluence does depend on the spectrum, however. Since from Equation V.20

$$
\Phi_{r}=\left(\frac{\bar{\sigma}_{C f}}{\bar{\sigma}_{r}}\right) k_{C f}{ }^{R}
$$

we can take a ratio between the $\Phi_{r}^{n}$ (calculated with the new calibration and new cross sections) and the $\Phi_{r}^{O}$ (calculated with the old calibration and old cross sections)

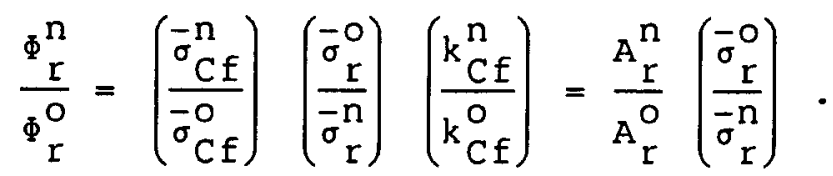

The ratio $\bar{\sigma}_{r}^{O} / \bar{\sigma}_{r}^{n}$ is the factor that makes the fluence correction different for each spectrum. 
The point of all this preliminary work is to show that, although sulfur activity calibrations carried out in 1980 by SNLA were correct and provided activities consistent with the activities of other reactions, the old cross-section set caused an error in the fluence determination. If we set $A_{r}^{n}=A_{r}^{O}$ (because activity does not depend on the cross-section set used) and substitute into Equation V.23, then one finds

$$
\frac{\Phi_{r}^{n}}{\Phi_{r}^{o}}=\frac{\sigma_{r}^{o}}{\sigma_{r}^{n}} .
$$

Below in Table V.28 are shown the old and new spectrumaveraged cross sections for all the SNLA configurations considered here.

Recent calibrations at NBS have verified that the activity calibration of sulfur dosimeters is in agreement with the older version.

The activities for sulfur and nickel obtained from the Radiation Dosimetry Laboratory and the SANDII spectra are listed in Table V.29. The listed spectrum-averaged cross sections, however, are based only on the calculated activities. For completeness, the spectral indices and the hardness parameters (discussed next) are included. 
Table V.28

Comparisons of Old and New Spectrum-Averaged Cross Sections

Source

$\bar{\sigma}_{r}^{o}(m b) \quad \bar{\sigma}_{r}^{n}(m b) \quad \bar{\sigma}_{r}^{o} / \bar{\sigma}_{r}^{n}=\Phi_{r}^{n} / \Phi_{r}^{o}$

SPRIII Bare Cavity

(BARNOB6)

SPRIII Polyethylene-Lined

Cavity (POLYCA48)

SPRIII Aluminum Liner

(RAN19)

SPRIII $17 "$ Leakage

(SLEAK21)

SPRIII old Cadmium-

Polyethylene Blocks (FLATCB24) 287

SPRIII Cadmium-Polyethylene +

Polyethylene Blocks (CDPOC7) 287

SPRIII Plugged Beam Shield

(BSHLD20)

SPRIII Beam Shield Aperature

(BESHLD 32)

ACRR Cavity Free Field

(ACRRCF 28)

ACRR Old Lead-Boron Bucket

(ACRPB14)

ACRR Fuel-Ringed External

Cavity (FREC2344)

ACRR New Lead-Boron Bucket

(LBACRR12)
285

328

0.870

285

323

0.882

285

328

0.869

287

320.5

0.895

323

0.889

287

317

0.905

287

348

0.825

287

331

0.867

323

339

0.953

323

350

0.923

323

325.5

0.992

323

348

0.928 
Table V.29

Sulfur and Nickel Spectrum-Averaged Cross sections, spectral Indices, and Hardness Parameters

\begin{tabular}{|c|c|c|c|c|c|c|c|}
\hline & $\begin{array}{c}3 \text { MeV } \\
\text { Fluence } \\
n / \mathrm{cm}^{2}\end{array}$ & $\begin{array}{l}\text { sulfur } \\
\text { Activity }\end{array}$ & Sulfur $\bar{\sigma}$ & $\begin{array}{l}\text { Nickel } \\
\text { Activity }\end{array}$ & Nickel $\bar{\sigma}$ & & Silicor \\
\hline $\begin{array}{c}\text { Geometry } \\
\text { (File Name) }\end{array}$ & SANDII & $\begin{array}{l}\text { Measured } \\
\text { Calculated }\end{array}$ & Calculated & $\begin{array}{c}\text { Measured } \\
\text { Calculated }\end{array}$ & Calculated & SI & HP \\
\hline $\begin{array}{l}\text { SPRIII Bare Cavity } \\
\text { (BARNOB6) }\end{array}$ & $3.588 \mathrm{E}+16$ & $\begin{array}{l}6.678 \mathrm{E}-18 \\
6.607 \mathrm{E}-18\end{array}$ & $328 \mathrm{mb}$ & $\begin{array}{l}2.010 \mathrm{E}-18 \\
1.968 \mathrm{E}-18\end{array}$ & $484 \mathrm{mb}$ & 8.46 & 0.889 \\
\hline $\begin{array}{l}\text { SPRIII Polyethylene } \\
\text { Lined Cavity } \\
\text { (PoLYCA48) }\end{array}$ & $8.561 \mathrm{E}+12$ & $\begin{array}{l}1.625 E-18 \\
1.552 E-18\end{array}$ & 323 & $\begin{array}{l}4.488 \mathrm{E}-19 \\
4.662 \mathrm{E}-19\end{array}$ & 481 & 8.39 & .764 \\
\hline $\begin{array}{l}\text { SPRIII Aluminum } \\
\text { Liner } \\
\text { (RAN19) }\end{array}$ & $1.101 \mathrm{E}+13$ & $\begin{array}{l}2.076 E-18 \\
2.025 E-18\end{array}$ & 328 & $\begin{array}{l}6.149 \mathrm{E}-19 \\
6.046 \mathrm{E}-19\end{array}$ & 485 & 10.40 & .870 \\
\hline $\begin{array}{l}\text { SPRIII 17" Free- } \\
\text { Field Leakage } \\
\text { (SLEAK21) }\end{array}$ & $2.615 E+12$ & $\begin{array}{l}4.680 \mathrm{E}-19 \\
4.705 \mathrm{E}-19\end{array}$ & 320.5 & $\begin{array}{l}1.402 \mathrm{E}-19 \\
1.407 \mathrm{E}-19\end{array}$ & 474 & 7.57 & .872 \\
\hline $\begin{array}{l}\text { SPRIII old Cadmium- } \\
\text { Polyethylene Blocks } \\
\text { (FLATCB24) }\end{array}$ & $1.834 \mathrm{E}+11$ & $\begin{array}{l}3.136 E-20 \\
3.328 E-20\end{array}$ & 323 & $\begin{array}{l}1.029 \mathrm{E}-20 \\
1.018 \mathrm{E}-20\end{array}$ & 490 & 6.42 & .868 \\
\hline $\begin{array}{l}\text { SPRIII Cadmium-Poly } \\
\text { ethylene + Poly- } \\
\text { ethylene Blocks } \\
\text { (CDPOC7) }\end{array}$ & $1.259 E+11$ & $\begin{array}{l}2.280 \mathrm{E}-20 \\
2.243 \mathrm{E}-20\end{array}$ & 317 & $\begin{array}{l}6.729 E-21 \\
6.765 E-21\end{array}$ & 474 & 7.96 & .745 \\
\hline $\begin{array}{l}\text { SPRIII Plugged } \\
\text { Beam Shield } \\
\text { (BSHLD20) }\end{array}$ & $4.196 \mathrm{E}+10$ & $\begin{array}{l}8.531 E-21 \\
8.201 E-21\end{array}$ & 348 & $\begin{array}{l}2.525 \mathrm{E}-21 \\
2.596 \mathrm{E}-21\end{array}$ & 546 & 18.8 & .669 \\
\hline $\begin{array}{l}\text { SPRIII Beam } \\
\text { Shield Aperture } \\
\text { (BESHLD32) }\end{array}$ & $8.248 \mathrm{E}+11$ & $\begin{array}{l}1.498 E-19 \\
1.533 E-19\end{array}$ & 331 & $\begin{array}{l}4.464 \mathrm{E}-20 \\
4.616 \mathrm{E}-20\end{array}$ & 494 & 8.76 & .858 \\
\hline $\begin{array}{l}\text { WSMR FBR Leakage } \\
\text { (WHSL 25) }\end{array}$ & $1.140 \mathrm{E}+12$ & $\begin{array}{l}2.076 \mathrm{E}-19 \\
2.024 \mathrm{E}-19\end{array}$ & 316 & $\begin{array}{l}5.906 \mathrm{E}-20 \\
6.066 \mathrm{E}-20\end{array}$ & 470 & 6.65 & .916 \\
\hline $\begin{array}{l}\text { ACRR Cavity Free } \\
\text { Field } \\
\text { (ACRRCF28) }\end{array}$ & $1.027 E+13$ & $\begin{array}{l}1.927 E-18 \\
1.957 E-18\end{array}$ & 339 & $\begin{array}{l}5.910 E-19 \\
5.740 E-19\end{array}$ & 493 & 12.46 & .726 \\
\hline $\begin{array}{l}\text { ACRR old Lead- } \\
\text { Boron Bucket } \\
\text { (ACRPB 14) }\end{array}$ & $7.114 \mathrm{E}+13$ & $1.397 \mathrm{E}-17$ & 350 & $\begin{array}{l}4.464 E-18 \\
4.262 E-18\end{array}$ & 528 & 16.45 & .705 \\
\hline $\begin{array}{l}\text { ACRR FREC } \\
(\text { FREC2344) }\end{array}$ & $4.344 \mathrm{E}+12$ & $\begin{array}{l}7.657 \mathrm{E}-19 \\
7.939 \mathrm{E}-19\end{array}$ & 325.5 & $\begin{array}{l}2.373 \mathrm{E}-19 \\
2.406 \mathrm{E}-19\end{array}$ & 489 & 9.07 & .778 \\
\hline $\begin{array}{l}\text { ACRR New Lead- } \\
\text { Boron Bucket } \\
\text { (LBACRR12) }\end{array}$ & $3.140 E+13$ & $\begin{array}{l}6.208 E-18 \\
6.145 E-18\end{array}$ & 348 & $\begin{array}{l}1.879 E-18 \\
1.854 E-18\end{array}$ & 521 & 16.83 & .659 \\
\hline $252 \mathrm{CE}$ & & & 321 & & 474 & 4.24 & 1.165 \\
\hline
\end{tabular}




\section{Silicon Displacement Damage}

The silicon displacement damage functions (or kerma factors) as a function of energy are reproduced in Table V. 30 from the ASTM standard E722-85.[32] This table can be used in the damage calculations described previously in Equation V.1.

$$
D=\int_{10 \mathrm{keV}}^{\infty} \phi(E) d(E) d E \equiv(E>10 \mathrm{keV}) \overline{\mathrm{d}} \text {. }
$$

The quantity $\bar{d}$ is often converted to a form which relates the damage produced by the spectrum $\phi(E)$ to that produced by $1 \mathrm{MeV}$ neutrons. One defines the damage by $1-\mathrm{MeV}$ neutrons to be $D_{1}$ and multiplies the numerator and denominator of Equation V.1 by this factor.

$$
\mathrm{D}=\Phi(\mathrm{E}>10 \mathrm{keV})\left(\frac{\overline{\mathrm{d}}}{\mathrm{D}_{1}}\right) \mathrm{D}_{1}
$$

The currently accepted value of $D_{1}$ is $95 \mathrm{MeV} \cdot \mathrm{mb} .[32]$ It was obtained by averaging the damage function in the vicinity of $1 \mathrm{MeV}$. For any other spectrum, the relative damage effectiveness can be determined in comparison with $1-M e V$ neutrons by integration with the kerma factors listed in Table V. 30 . The resulting parameter is called the hardness parameter, HP.

Thus

$$
\mathrm{HP}=\frac{\overline{\mathrm{d}}}{\mathrm{D}_{1}}
$$

$$
\mathrm{D}=\Phi(\mathrm{E}>3 \mathrm{MeV}) \cdot \mathrm{SI} \cdot \mathrm{HP} \cdot \mathrm{D}_{1} \equiv \Phi(1 \mathrm{MeV}) \cdot \mathrm{D}_{1}
$$

where

$\Phi(1 \mathrm{MeV})=$ equivalent $1 \mathrm{MeV}$ fluence for this spectrum

$$
\Phi(E>3 \mathrm{MeV})=\frac{\mathrm{A}}{\mathrm{N} \lambda \bar{\sigma}(\mathrm{E}>3 \mathrm{MeV})}
$$

HP = hardness parameter.

The hardness parameters for the 13 measured spectra are listed in Table V.29. 


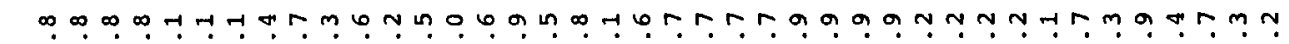

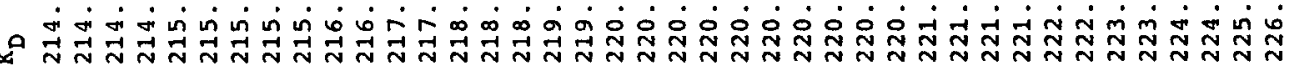

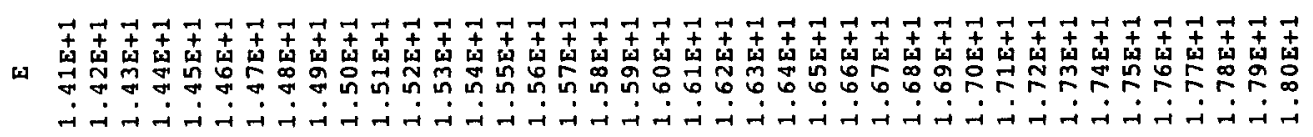

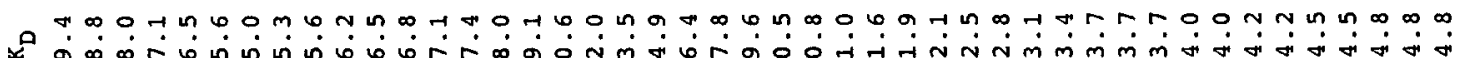

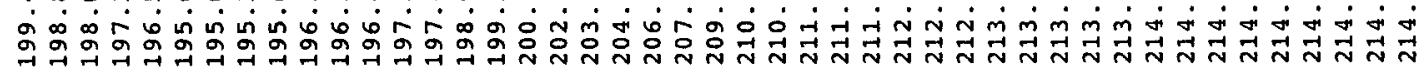

.5

되

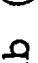

皇

$\dot{\mathrm{d}}$

.

a

$\ddot{a}$

옹

O

山 बंबव

mand o

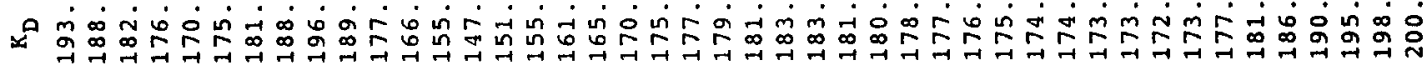

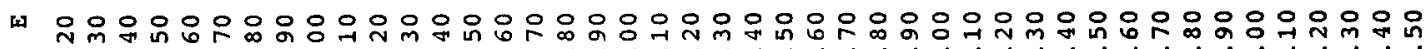
n n

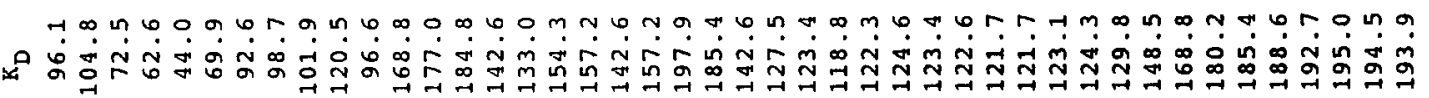

at

$\hat{l}$

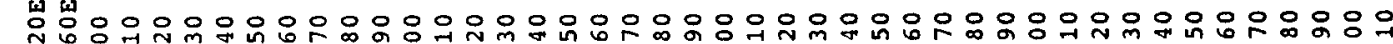

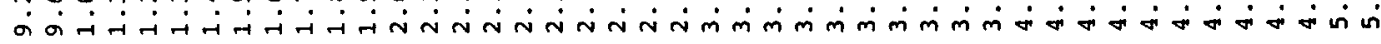

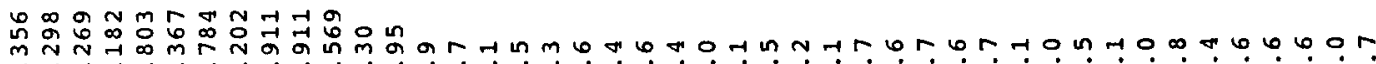

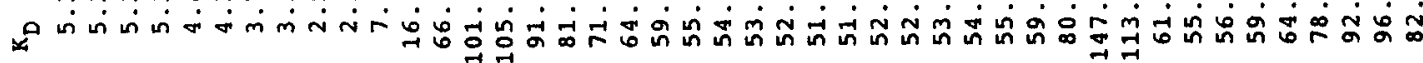

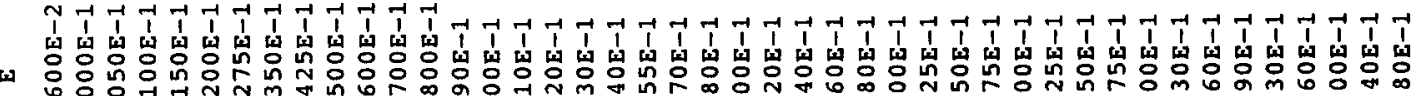
ब-

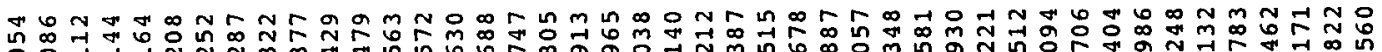
*

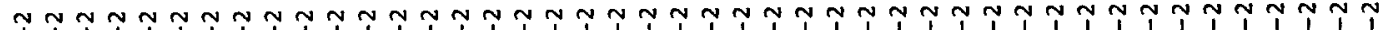

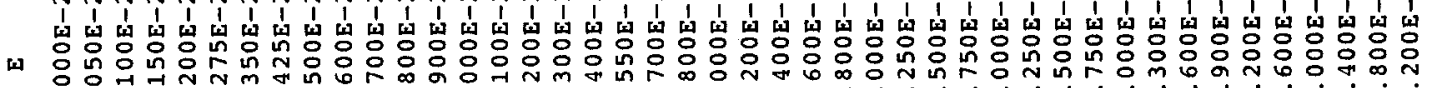

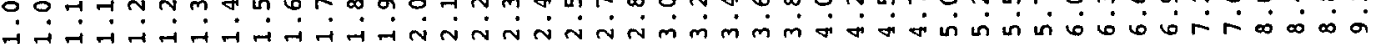


Just as the spectrum-averaged cross section for sulfur is not very sensitive to the spectral shape, the hardness parameter does not vary a great deal over this set of spectra measurements. In fact, the only justification for breaking up the damage function integral or the spectrum-averaged cross section integral into the products of various factors as described above is that the various factors do not vary by large amounts between realistic spectra. The analyst is really only justified in using these factors in environments that have been well characterized already. In each of these cases, the integrations must have been carried out over the correct spectrum. If one attempts to use a data base such as the 13 spectra described in this report to make estimates for another environment, one risks making serious errors. 


\section{E. Comparisons with Other spectra}

At this time we do not have experimentally determined spectra for the SPR III that have been obtained by other means or by other experimenters. However, comparisons can be made to the spectra measured at the SPR II and the WSMR FBR. Although SPR III is a larger reactor with a larger experimental cavity than SPR II or the WSMR FBR, it is nevertheless a fast-burst reactor of the Godiva type and should exhibit similarities in the shape of its spectrum.

Plotted in Figure V.27 are three cavity spectra that have been normalized at $1 \mathrm{MeV}$. The first one is that determined by the foil measurements and SANDII, reported for the SPR III free-field cavity spectrum given in this report (spectrum \#1). The scott SPRII cavity spectrum was measured by the foilactivation technique and was reported in Reference 3. Since the primary data were not available, the fluence values were read from the small graphs in the publication. The third spectrum was obtained in a similar fashion from curves for the SPR II cavity spectrum published by Powell in 1971.[33] His spectrum was obtained in an entirely different manner, however, being a composite of proton recoil measurements at the lower end of the spectrum and of fission thermocouple measurements taken at higher energies.

The apparent large differences between the curves are exaggerated by the choice of the normalization energy. As mentioned before, the higher peak in Curve 1 at $0.3 \mathrm{MeV}$ and lower fluence at $0.03 \mathrm{MeV}$ resulted from the search for a smooth curve compatible with the activities of ${ }^{24} \mathrm{Na},{ }^{2} 39 \mathrm{Pu}$, and $235 \mathrm{U}$. We believe that this structure is real, because the same tendency has appeared in some but not all of our other spectra. The thermal tail on the SPR III free-field measurement (Curve 1) has been well established and should certainly be much larger than that of SPR II, which has smaller openings at the ends of the cavity, through which thermal neutrons can enter. Neither of the other two measurement configurations was sensitive at low neutron energies.

In Figure V.28 are plotted leakage spectra from the same three sources, with the addition of the spectrum we obtained at the WSMR FBR (spectrum \#9). The spectrum \#9 was not normalized to the others at $1 \mathrm{MeV}$. Curves 1 and 2 both exhibit the onset of a thermal tail below $0.01 \mathrm{MeV}$ from the kiva walls. The tail is expected to be larger for Curve 1 on SPR III, because measurements were made further from the core axis. We don't believe, however, that the tail on scott's spectrum (Curve 2) was well established by the foil set he used (no gold) and therefore may simply be the remnant of the calculated trial that would not have been altered by a set of activities insensitive in that energy region. 


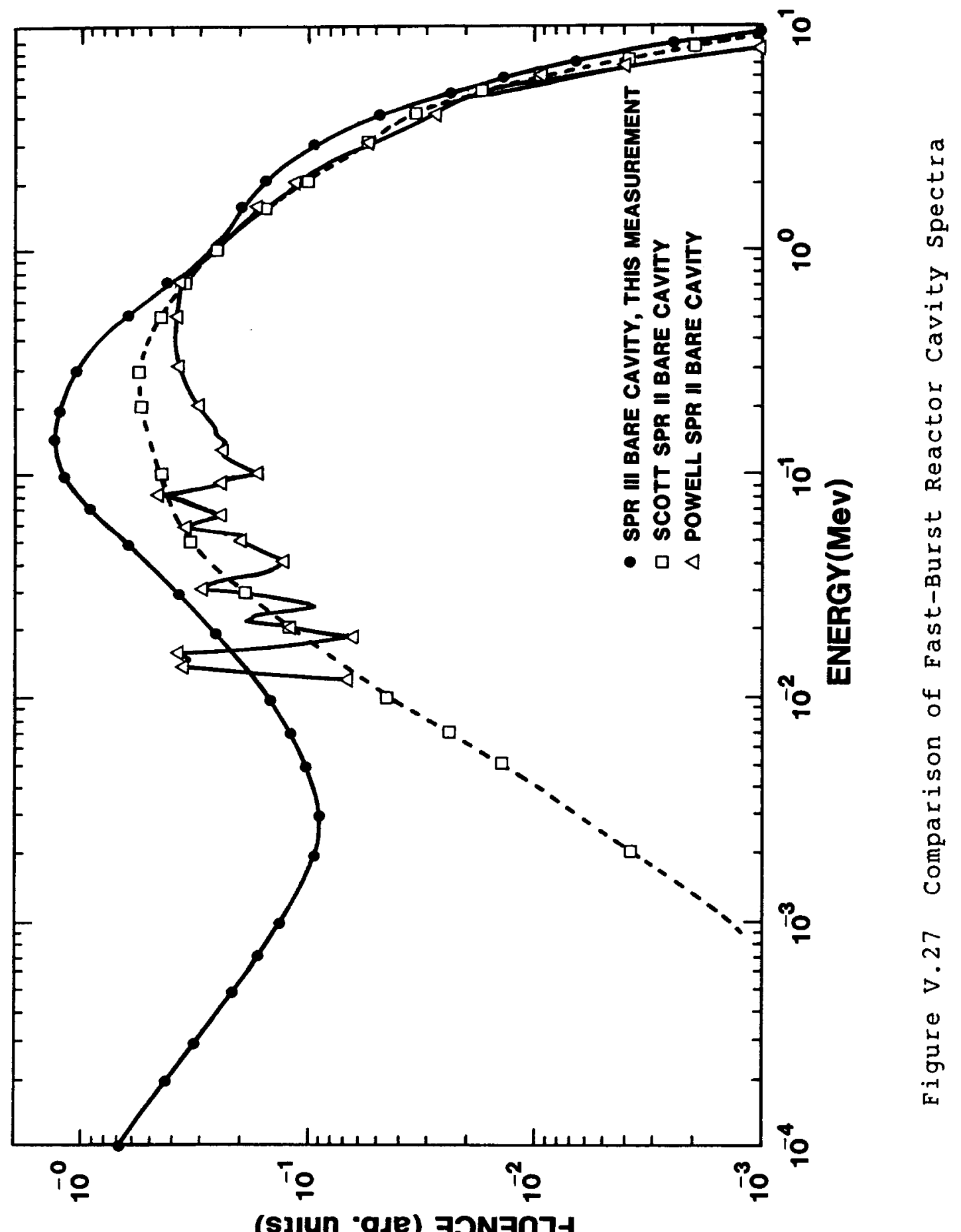




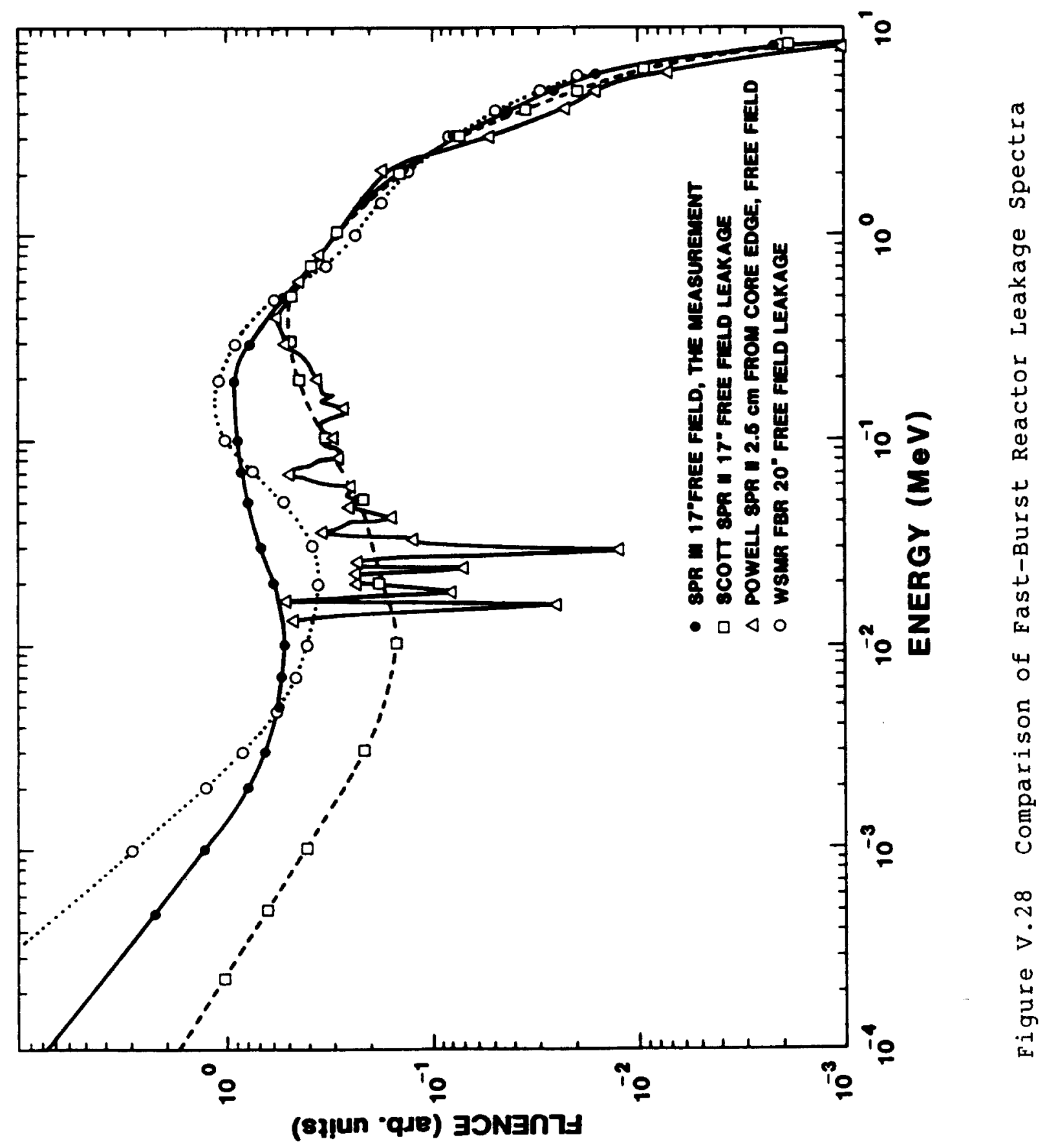


One naturally must ask whether the structure in the proton recoil spectra in both sets of data could be real since there is much $235_{\mathrm{U}}$ in the vicinity, with its strong resonance structure at low energies. There does not appear to be any correlation in the peaks and valleys of the curves in the two figures, however, and the resonance structure of $235_{U}$ only becomes large below about 1E-4 MeV. We have smoothed these curves for the calculation of spectral indices and damage functions.

Each of these curves has been reduced to a table of fluence values at discrete energies and inserted as a trial function in SANDII. The code was then run with an iteration limit of 0 . This means that the shape of the trial in each case is preserved and only the normalization is changed. The code then provides a spectral index and a spectrum that, when used in the program DAMAGE, calculates the hardness parameter for silicon. Table V.31 contains the spectral indices and hardness parameters for each of the curves in the two figures.

The hardness parameters are in fair agreement ( $\sim 8 \%$ ) for the cavity spectra, and the spectral indices are also quite close to each other. For the larger SPR III core and with more leakage into the cavity, it is not surprising that its SI is slightly higher. For the leakage spectra the hardness parameter of SPR III is lower, as expected, since the measurements were made at a greater distance from the core surface. The large disagreement in SI for the Powell SPR II leakage spectrum is also not surprising since this spectrum is a composite pieced together at a point near the fulcrum of the $S I$ ratio. In general the agreements seen here are better than one might expect from curves read from published graphs. The data confirm the relative insensitivity of the hardness parameter to the details of spectrum shape. 
Table V.31

Spectral Indices and Hardness Parameters From Three Fast-Burst Reactors

\begin{tabular}{|lcccc|}
\hline Type & Source & & $\begin{array}{c}\text { Spectral } \\
\text { Index }\end{array}$ & $\begin{array}{c}\text { Hardness } \\
\text { Parameter }\end{array}$ \\
\hline Cavity & SPR II & Kelly & 8.46 & .889 \\
Cavity & SPR II & Scott & 8.22 & .918 \\
Cavity & SPR II & Powell & 8.47 & .965 \\
Leakage & SPR III & Kelly & 7.6 & .872 \\
Leakage & SPR II & Scott & 7.71 & .973 \\
Leakage & SPR II & Powell & 10.80 & .957 \\
Leakage & WSMRFB & Kelly & 6.63 & .916 \\
\hline
\end{tabular}




\section{F. Errors}

It may be painfully evident to the reader that the analysis of errors has been inadequate, particularly in the development of criteria with which one can evaluate the accuracy of a differential fluence value. That, of course, would be the ultimate achievement. However, we argue that this is an unrealistic goal for an underdetermined problem. There is a full range of cross sections to deal with, all of which are more accurately defined at some energies than at others. This means that the influence of these unknown errors will be different for different spectra. One can, of course, make estimates of the errors at each energy or rely on published measurement errors when they are available, but there are going to be large uncertainties in the errors themselves. The analyst cannot get around the fact that the principal indicators of spectrum character from, say, 15 reactions are 15 single numbers, the activities for each reaction.

One does not care so much about a spectrum error at a particular energy as much as how the errors may influence integral quantities, such as the damage induced in silicon. In this context, investigation of errors by sensitivity analysis is entirely justified.

Consider the example of the influence of the manganese foil. Its activity could be erroneous because of counting statistics or experimental error, or its influence on the adjustment procedure may be incorrect because the tabulated cross section is wrong. In chapter IV, we found that a 5\% variation of the MN55G activity caused a $30 \%$ change in the differential fluence at $10 \mathrm{kev}$. The experimenter may not be disturbed by this if he/she were interested only in the damage the fluence causes in a silicon device. From section $D$ in this chapter, one can determine the damage in silicon with equation V.28:

$$
D=\Phi(E>3 \mathrm{MeV}) \cdot \mathrm{SI} \cdot \mathrm{HP} \cdot \mathrm{D}_{1} .
$$


All of the parameters can be determined from the SANDII spectra. We have run the code for the SPR III cavity spectrum with an MN55G activity raised by 15\% (an amount by which we originally decreased it because of an estimated cross-section error in the old cross-section set).

\begin{tabular}{|c|c|c|c|c|c|}
\hline Activity & $\phi(E>3 \mathrm{MeV})$ & SI & $\begin{array}{l}D \underset{H P}{(A} \\
\end{array}$ & $\begin{array}{l}\text { Units) } \\
\text { Un }\end{array}$ & Difference \\
\hline$A^{\prime}$ Mn (measured) & $4.968 E+13$ & 8.73 & .928 & 3.821 & \\
\hline$A^{\prime} M n \quad \begin{array}{c}(\text { measured } \\
15 \%)\end{array}+$ & $4.994 \mathrm{E}+13$ & 8.81 & .915 & 3.824 & $.09 \%$ \\
\hline
\end{tabular}

The calculated damage function differs by only 0.09\%. (Note: this comparison and the next were made with parameters from an older cavity spectrum.)

Let us try another example with a foil that may influence the damage function more strongly. The sulfur pellet for which the activity may be $7 \%$ high. Lower the sulfur activity by $10 \%$. Then

\begin{tabular}{|c|c|c|c|c|c|}
\hline Activity & $\phi(\mathrm{E}>3 \mathrm{MeV})$ & $\mathrm{SI}$ & $\mathrm{h}_{\mathrm{p}}$ & $\begin{array}{c}D(\text { Arb.) } \\
\text { Units) } \\
\end{array}$ & Difference \\
\hline (measured) & $4.968 E+13$ & 8.73 & .928 & 3.821 & \\
\hline $\begin{array}{l}\text { (measured } \\
-10 \%)\end{array}$ & $4.809 E+13$ & 9.02 & .923 & 3.803 & $0.47 \%$ \\
\hline
\end{tabular}

The calculated damage is reduced by $0.47 \%$. This is partly because lowering the sulfur activity lowers $\phi(E>3 \mathrm{MeV})$ but raises SI and $\phi(E>10 \mathrm{keV})$ is changed very little.

The indication is clear that changing the activity of one foil by amounts we believe are appropriate for the errors makes very little change in the hardness parameter, the $\Phi(E)$ $10 \mathrm{keV})$, or the damage itself. In addition, if there were some common mode calibration error that raised all the activities by some amount, neither the spectral shape nor the hardness parameter would be altered. Only the normalization of $\Phi(E>10 \mathrm{keV})$ would be affected.

The upshot of this discussion is that, although a $15 \%$ error in the manganese cross section may cause a $100 \%$ shift in the spectrum at $10^{-2} \mathrm{MeV}$, but this would have little effect on the damage in silicon. If the experimenter is concerned about 
activation in gold and there is a $10 \%$ error in the gold activity, then perhaps he will have a $10 \%$ error in his experiment because the gold response is somewhat isolated.

The primary concern for most of the experimenters that use these facilities will be the accuracy of the overall average calibration of the Radiation Dosimetry Laboratory for the foil set. The integral fluences $\Phi(E>3 \mathrm{MeV})$ on the basis of Chapter III should now be correct to 5\%. Similar claims can be made for $\phi(E>10 \mathrm{keV})$ and silicon damage because $S I$ and $\mathrm{HP}$ should be insensitive to these calibrations.

Thus it appears that fairly crude estimates of most of the potential errors coupled with some sensitivity studies can lead to very good determinations of the error bounds of the relevant functions. However, a full range of errorsensitivity studies has not yet been undertaken at this laboratory. 
A procedure has been developed that permits a practiced analyst to obtain acceptable neutron spectra in reactor facilities without any a priori knowledge of the form of each spectrum. The spectra are obtained by applying the SANDII code to a consistent set of foil activities obtained from the exposure of materials, selected for a sufficient breadth of responses, to a neutron radiation environment. Based on the SANDII results, a new trial is developed which is derived by a simple smoothing operation of the previous result. The analyst further develops the trial function by guiding it close to the previous result where the response of the foil set is high. An intimate knowledge of the response functions also aids in the interpretations of the SANDII results for construction of a better trial. Ultimately, when the trial is close to agreeing with all the activities from the start of the iteration procedure, the code simply verifies a good trial. The important point is that for the spectra adjusted thus far, very close to the same spectrum has been obtained for a given foil set no matter what form the trial function had at the beginning of the analysis. Furthermore, since one can begin the procedure with the same trial in all spectra to be analyzed, the differences that result must be dependent only on the real differences among the foil-set activities. SANDII gives excellent clues that can lead to the improved trials.

We have also investigated the rigidity of spectrum solutions obtained at each energy by measuring the rate with which SANDII will reestablish a solution whose trial has been locally perturbed positively and negatively in a smooth manner. It has been found that the adjusting operation with perturbed trials is not very successful when: (1) the trial is perturbed in a region of very small foil response, and (2) when errors have been made in the activity determinations so that there are conflicts in fitting a reasonable spectrum to the various activities. If the foil set is compatible in the sense that serious conflicts are absent, then the shape in low response regions can often be determined quite well by allowing the code to fit the activities, then smooth, then fit again until very low standard deviations of the measured to calculated activities are obtained. In this mode the analyst also depends on the neighboring regions of higher response to construct better trials. The perturbation method is not very useful in establishing an "error band" versus energy or a solution "rigidity" in the regions of low response.

Obviously, the problem of low response can be reduced by identifying and using improved sets of reactions that provide better coverage. The results are improved even more by making 
careful measurements to reduce conflicts that we know cannot exist in accurately measured activity sets. For this latter case, accurate, up-to-date, reaction cross-section information is very important.

In Chapter $V$, the results of spectrum determinations in a wide variety of environments have been presented. This compilation was undertaken because of the increasing variety of geometries used by experimenters in these facilities. Most of them also wish to be able to incorporate simple dosimetry systems, such as sulfur pellets, into their experiments. In order for this procedure to be meaningful, the spectra in these environments must be accurately known, and the sulfur pellet dosimetry (or other system) calibration must be established. In situations in which the spectra cannot be measured, the benchmark calculations that have been conducted show that MORSE can provide spectra that are in reasonable agreement with measured spectra.

The comparisons among many spectra and activity sets have been very illuminating, because they were all generated with the same procedures. The tendency for the IN $115 \mathrm{~N}$ to be high on all data sets led to the recognition of the potential gamma-ray excitation of this reaction. The FE56P reaction is high in all instances, indicating either cross-section uncertainties or real spectrum structure. A shift in the direction of fission-foil deviations in cavity and leakage spectra, indicates real structural differences, and there were numerous other correlations that justify investigation. These tendencies would not have been meaningful without the availability of a broad range of data sets.

We have tried to include sufficient information about the activity sets, the spectra, and the procedures so that this report will be useful to experimenters preparing for tests and to analysts investigating adjustment procedures. For those who are interested in neutron radiation damage in electronic devices, the hardness parameter, HP, has been included in Table V.29. The explanations accompanying that table show how the various parameters that are derived from the spectra determinations can be used to calculate the damage in silicon devices generated by the various spectra.

There is much that must be done before this work is finished, and certain activities will, of course, be continued as long as reactor experiments are conducted at SNLA. Spectra are needed in and around the SPR II reactor and in various filter structures at the ACRR and in its external cavity. We believe that some important reaction cross sections require updating and remeasurement. The experiments have shown that the boron balls have more influence on the response functions of materials than is predicted by the exponential attenuation model of SANDII or the experimental corrections used by WSMR personnel for their reactor. We are convinced that just using 
the boron absorption cross section is not really correct, but this practice is much better than using the total cross section. The proper cross section must be a compromise between these two, with the addition of carbon scattering.

Comparisons with the results obtained from other codes are also important. Many of the shortcomings of SANDII that other investigators have pointed out are the same as those we have mentioned here.[34] Although knowing the uncertainties is important, it is even more important to experimentally reduce relative uncertainties between measured activities within a foil set. When this is accomplished, SANDII in particular can generate very well-defined spectral information.

Calibration work will continue at a high level at this laboratory, and we plan to increase the number of interlaboratory comparisons. This document is far from being the last word on neutron spectra measurements at SNLA, and it is strongly recommended that any experimenter using the facilities here confer with the authors, with Radiation Dosimetry Laboratory personnel, and/or with the facility operators regarding the environments he or she will encounter. 


\section{REF ERENCES}

1. S. Berg and W. N. McElroy, A Computer-Automated Iterative Method for Neutron Flux Spectra Determination by Foil Activation, AFWL-TR-67-41, Atomics International, sept. 1967.

2. C. R. Greer, J. A. Halblieb, and J. V. Walker, "A Technique for Unfolding Neutron spectra from Activation Measurements," SC-RR-67-746, Sandia National Laboratories, Albuquerque, NM, 1967.

3. V. V. Verbinski, N. A. Lurie, and V. C. Rogers, "Threshold-Foil Measurements of Reactor Neutron Spectra for Radiation Damage Applications," Nuclear Science and Engineering, 65, 1978, pp. 316-330.

4. F. G. Perry, Least Squares Dosimetry Unfolding: The Program STAY'SL, ORNL/TM-6062, ENDF-254, Oak Ridge National Laboratory, Oak Ridge, TN, 1977.

5. F. Schmittroth, FERRET Data Analysis Code, HEDL-TME 79-10, Hanford Engineering Development Laboratory, Richland, WA, Sept. 1979 .

6. B. K. Kam and F. W. Stallmann, CRYSTAL BALL-A Computer Program for Determining Neutron Spectra from Activation Measurements, ORNL/TM-4601, Oak Ridge National Laboratory, Oak Ridge, TN, June 1974.

7. J. T. Routti, LOUHI, A Computer program for the Numerical solution of Feldholm Integral Equations of the First Kind, Lawrence Berkeley Laboratory, Berkeley, CA, 1970 .

8. L. L. Bonzon, F. M. Morris, and F. V. Thome, Annular Core Pulse Reactor (ACPR): Experimenter's Manual, SLA73-1017, Sandia National Laboratories, Albuquerque, NM, oct. 1974 .

9. B. F. Estes and J. A. Snyder, Sandia Pulsed Reactor Facility (SPRF), SAND79-0391, Sandia National Laboratories, Albuquerque, NM, June 1979.

10. S. A. Dupree and R. E. Lighthill, Sandia National Laboratories CDC7600 Version of MORSE-SGC, SAND80-1337, Sandia National Laboratories, Albuquerque, NM, Feb. 1982 .

11. R. F. Sartor, "Monte Carlo Determination of the Neutron-Gamma spectrum Behind Cadmium Loaded Polyethylene Slabs Irradiated by the Sandia Pulse Reactor III," (Master's thesis, Texas A\&M University, College Station, TX, May 1986). 
12. Developed for the Radiation Dosimetry Laboratory by Atomergic Chemetals, Plainview, NY.

13. APOGEE Technical Reference Manual, CISE 711, Canberra Industries, Inc., Meriden, CT, 1985.

14. M. J. Koskelo, P. A. Aarnio, and J. T. Routti, "SAMPO80: An Accurate Gamma Spectrum Analysis Method for Minicomputers," Nucl. Instr. Meth., 190, p. 89, 1981 .

15. "Product Specification: Mixed Radionuclide Gamma-Ray Reference Source, Code QCD.1," Amersham Corporation, Arlington Heights, IL, 1985.

16. B. F. Rider and M. E. Meek, Compilation of Fission Product Yields, NEDO-12154-2(D), Vallecitos Nuclear Center, Pleasanton, CA, July 1977.

17. V. V. Verbinski, C. Cassapakis, and H. L. scott, "Transistor Damage Characterization by Neutron Displacement Cross section in Silicon: Experimental," Nuclear Science and Engineering, 70, pp. 66-72, 1979.

18. J. E. Powell and J. W. Mathews, "A Proton Recoil spectrometer for Neutron spectrum Measurements in the Sandia Pulsed Reactor Facility," SC-RR-71-0453, Sandia National Laboratories, Albuquerque, NM, 1971, (plus later publications).

19. W. L. Zijp, H. J. Nolthenius, H. Ch. Rieffe, "Multigroup Cross Sections in SANDII Format for spectral, Integral, and Damage Analyses," DOSDAM81-82, DLC-97, Oak Ridge National Laboratory, RSIL Data Library Collection, Oak Ridge, TN, Dec. 1981 .

20. R. E. MacFarlane, D. W. Muir, and R. M. Boicourt, NJOY Nuclear Data Processing System, LA-9303-M, Vol. I and Vol. II (ENDF-324), Los Alamos National Laboratory, Los Alamos, NM, May 1982 .

21. W. Sallee, White Sands Missile Range, NM, private communication, 1985.

22. J. T. Harvey, J. L. Meason, and H. L. Wright, "Neutron Attenuation due to Boron in Shields," Nuclear Science and Engineering, 67, pp. 343-344, 1978 .

23. J. Meason, private communication, White Sands Missile Range, NM, 1982 .

24. R. F. Sartor, private communication, Texas A\&M University, College station, TX, Nov. 1985. 
25. K. R. Boldt et al., Sandia Annular Core Research Reactor (ACRR) Safety Analysis Report," SAND77-0208, Sandia National Laboratories, Albuquerque, NM, April 1978 .

26. W. Sallee, private communication, White Sands Missile Range, NM, 1985.

27. J. Meason, private communication, white Sands Missile Range, NM, 1985.

28. W. L. Zijp et al., Cross section Library DOSCROS77 (in the SAND-II format) ECN-25 Netherlands Energy Research Foundation, ECN, Petten, August 1977.

29. J. Grundl and C. Eisenhower, "Fission Rate Measurements for Materials Neutron Dosimetry in Reactor Environments," Proceedings 1st ASTM-EURATOM symposium on Reactor Dosimetry, Sept. 1975 .

30. American society for Testing and Materials (ASTM) Standard E265-82, Vol. 12.02, Philadelphia, PA, 1982.

31. E. D. McGarry, private communication, Bldg. Phy-A106, National Bureau of standards, Washington, DC.

32. A S T M Standard E722-85, Vol 12.02, Philadelphia, PA, 1985 .

33. J. E. Powell, High Resolution Neutron Spectrum Measurements in the sandia Pulsed Reactor-II Facility, SC-DC722287 , Sandia National Laboratories, Albuquerque, NM, Aug. 1972 .

34. D. W. Wootan and F. Schmittroth, Comparison of SANDII and FERRET, HEDL-TC-1589, Hanford Engineering Development Laboratory, WA, Jan. 1981. 


\section{Appendix A \\ The Perturbation Function}

We seek a function that will perturb the solution spectrum in a smooth manner in log-log space and in a limited energy region, say from $E_{b}$ to $E_{f}$. It is beneficial to force the net change in total fluence to be zero over the interval in which the fluence is perturbed, because then the normalization of the fluence outside that interval will be undisturbed, and it will be easier to determine the local effects of the perturbation.

Suppose that

$$
\Phi_{T}=\int_{0}^{\infty} \phi(E) d E
$$

where $\phi$ is the differential fluence, $\mathrm{n} / \mathrm{cm}^{2}$.

Define a new differential fluence, $\phi^{\prime}(E)=P(E) \Phi(E)$, that is perturbed from the original fluence only in the region $E_{b} \rightarrow E_{f}$. If we require that $\Phi_{T^{\prime}}=\Phi_{T}$, then

$$
\int_{E_{b}}^{E_{f}} \phi(E)[1-P(E)] d E=0 \text {. }
$$

We have arbitrarily chosen a form for $P(E)$ which is sinusoidal in log space:

$$
P(E)=1+C(E) \sin w\left(x-x_{b}\right)
$$

where $x=\log E$.

Forcing $P(E)$ to equal 1 at $E_{b}$ and $E_{f}$, requires

$$
\sin W\left(\log E_{f}-\log E_{b}\right)=0 \text {. }
$$

Then

$$
W=\frac{2 \pi}{\log \frac{E_{f}}{E_{b}}}
$$


and

$$
P(E)=1+C(E) \sin 2 \pi \frac{\log \frac{E}{E_{b}}}{\log \frac{E_{f}}{E_{b}}}
$$

From Eq. A.2,

$I=\int_{E_{b}}^{E_{f}} \phi(E)[1-P(E)] d E=\int_{E_{b}}^{E_{f}} \phi(E) C(E) \sin 2 \pi \frac{\log \frac{E}{E_{b}}}{\log \frac{E_{f}}{E_{b}}} d E=0 .(1.7)$

It would be desirable to satisfy this restriction no matter what the form of $\phi(E)$ is. Therefore, we define $C(E)$ to be the following:

$$
C(E)=\frac{B E b}{E \phi(E)}
$$

where $B=$ constant.

This makes $I=0$.

The form of $P(E)$ can be derived as follows:

$$
I=\int_{E_{b}}^{E} \phi(E) \quad\left(\frac{B E_{b}}{E \phi(E)} \sin 2 \pi \frac{\log \frac{E}{E_{b}}}{\log \frac{E_{f}}{E_{b}}}\right) d E
$$

let

$$
\begin{gathered}
\frac{\log \frac{E}{E_{b}}}{\log \frac{E_{f}}{E_{b}}} \equiv \frac{\log \frac{E}{E_{b}}}{\alpha} \equiv x \\
\frac{d x}{d E}=\frac{1}{\alpha} \frac{E_{b}}{E}
\end{gathered}
$$


but

$$
I=\alpha \int_{0}^{1} \sin 2 \pi x d x
$$

Thus, the form of the perturbation function becomes

$$
P(E)=1+B \frac{E_{b}}{E \phi(E)} \sin 2 \pi \frac{\log \frac{E}{E_{b}}}{\log _{\frac{E}{E_{b}}}} \text {. }
$$




\section{Appendix B}

Intersection Point of Perturbed Solutions

The objective of this calculation is to find the value of the fluence to which SANDII would settle at energy $E$ if the trial spectrum is first perturbed upward by a fraction $\delta$ and then downward by the same fraction from an initial value of $\phi_{0}$. We do this in the simplest manner by assuming that the solutions approach each other linearly as a function of the number of iterations and by observing where they are after $\alpha$ iterations. The geometry in iteration-fluence space (at $E$ ) is shown in Figure B.1.

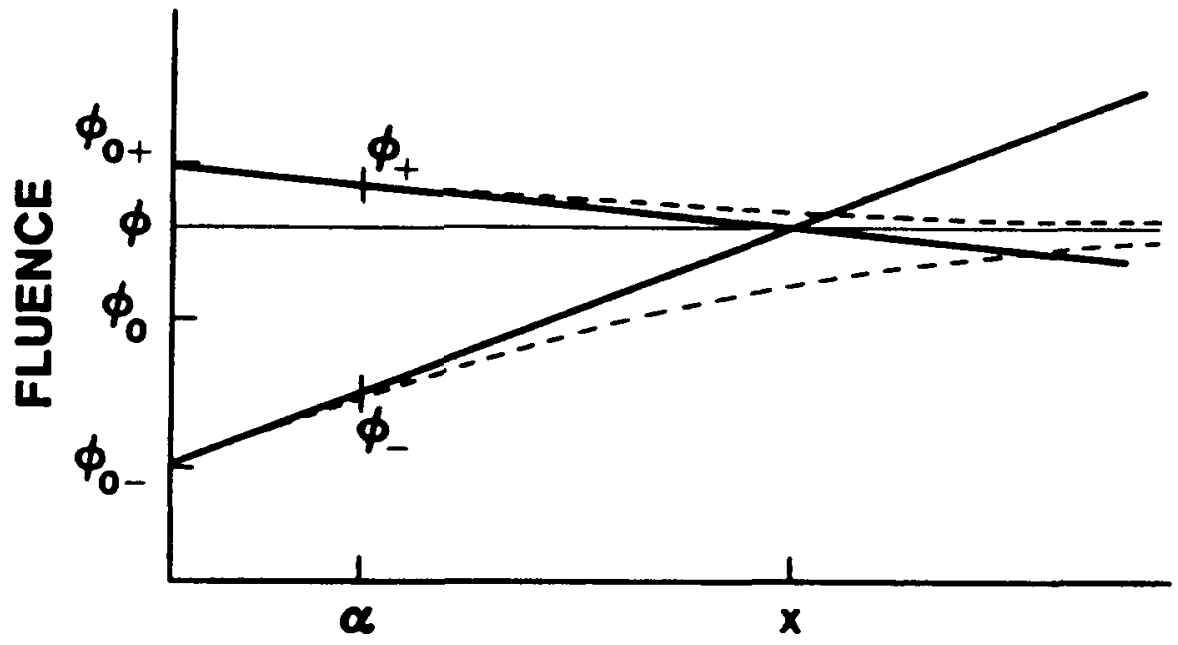

ITERATIONS

Figure B.1. Solution Trajectories in Iteration space

The figure shows that $\phi \neq \phi_{0}$ because we do not know whether the $\phi_{O}$ at this time was the correct value of $\phi$. Generally, $\phi$ will not equal $\left(\phi_{+}+\phi_{-}\right) / 2$

$$
\begin{gathered}
\phi_{O+} \equiv \phi_{0}(1+\delta) \\
\phi_{O-} \equiv \phi_{O}(1-\delta) \\
\phi=\frac{\phi_{+}-\phi_{O+}}{\alpha} x+\phi_{0}(1+\delta)
\end{gathered}
$$


and

$$
\phi=\frac{\phi_{-}-\phi_{0-}}{\alpha} x+\phi_{0}(1-\delta)
$$

By eliminating $x$, the number of iterations, from Eq. B. 3 and B.4, the fluence at the intersection point of the two lines is established.

$$
\phi=\phi_{0} \frac{\phi_{-}(1+\delta)-\phi_{+}(1-\delta)}{\phi_{-}+2 \phi_{0} \delta-\phi_{+}} \text {. }
$$

How many iterations does it take to reach $x$ if $\alpha$ iterations were needed to reach $\alpha$ ? Using Eqs. B.3 and B. 4 ,

$$
\frac{x}{\alpha}=\frac{2 \delta \phi_{0}}{\phi_{-}+2 \phi_{0} \delta-\phi_{+}} .
$$

In regions of weak response, $x / \alpha$ can be quite large, and even very small errors can lead to large errors in $\phi$, especially if $\phi_{0}$ was a poor guess.

One other comment should be made. The solution trajectories will never cross at $x$ but would tend to approach each other exponentially, as suggested by the dashed lines in Figure B.1. However, it is easy to prove that exponential trajectories will also approach the same value of $\phi$ at infinite $x$. Equation 4.7 still provides some measure of how many times one would need to iterate. 


\author{
Appendix C \\ Normalization of Fission-Foil Activities
}

It was conjectured that the ratio of activities for nickel exposed inside and outside the boron ball that contains the fission foils should have little dependence on the spectrum shape. This is confirmed by the second column in Table C.1. Each of the spectra obtained in an earlier SANDII run was used as an input spectrum for an activity run with the code. It can be seen from that column that indeed the calculated ratio for any of the spectra varies by, at most, 2 \%. In contrast to this, the measured ratios listed in the third column varied by much larger amounts.

Although SANDII does not correctly calculate the attenuation of the boron ball, its indication that attenuation varies only slightly with spectrum should be correct. Therefore, the variation in the measured ratio must be accounted for primarily by variations of placement of the ball foils with respect to the free-field foils. It was assumed that the average value of the ratio for all the foil sets would represent the correct value of the ratio (rather than the incorrectly calculated one). The highest and lowest ratios were deleted, and the average ratio was determined for the rest. This turned out to be $0.894 \pm 0.034$.

The fourth column in Table C.1 shows the correction factor, $f_{i}$, that was applied to the activities of the fissionfoil set for each spectrum. This factor forces the nickel activity ratios to be 0.894 for each geometry.

$$
f_{i}=\frac{0.894}{R_{i}} \text { (measured) }=\begin{aligned}
& \text { correction factor for boron ball } \\
& \text { activities } .
\end{aligned}
$$


Table C.1

Corrections to Fission-Foil Activities Based on Nickel-Foil Ratios

$$
R_{i}=\frac{A_{N i}(\text { in boron ball })}{A_{N i}(\text { free field })}
$$

spectrum

Name
$R_{i}$

(Measured) $\mathbf{f}_{i}$

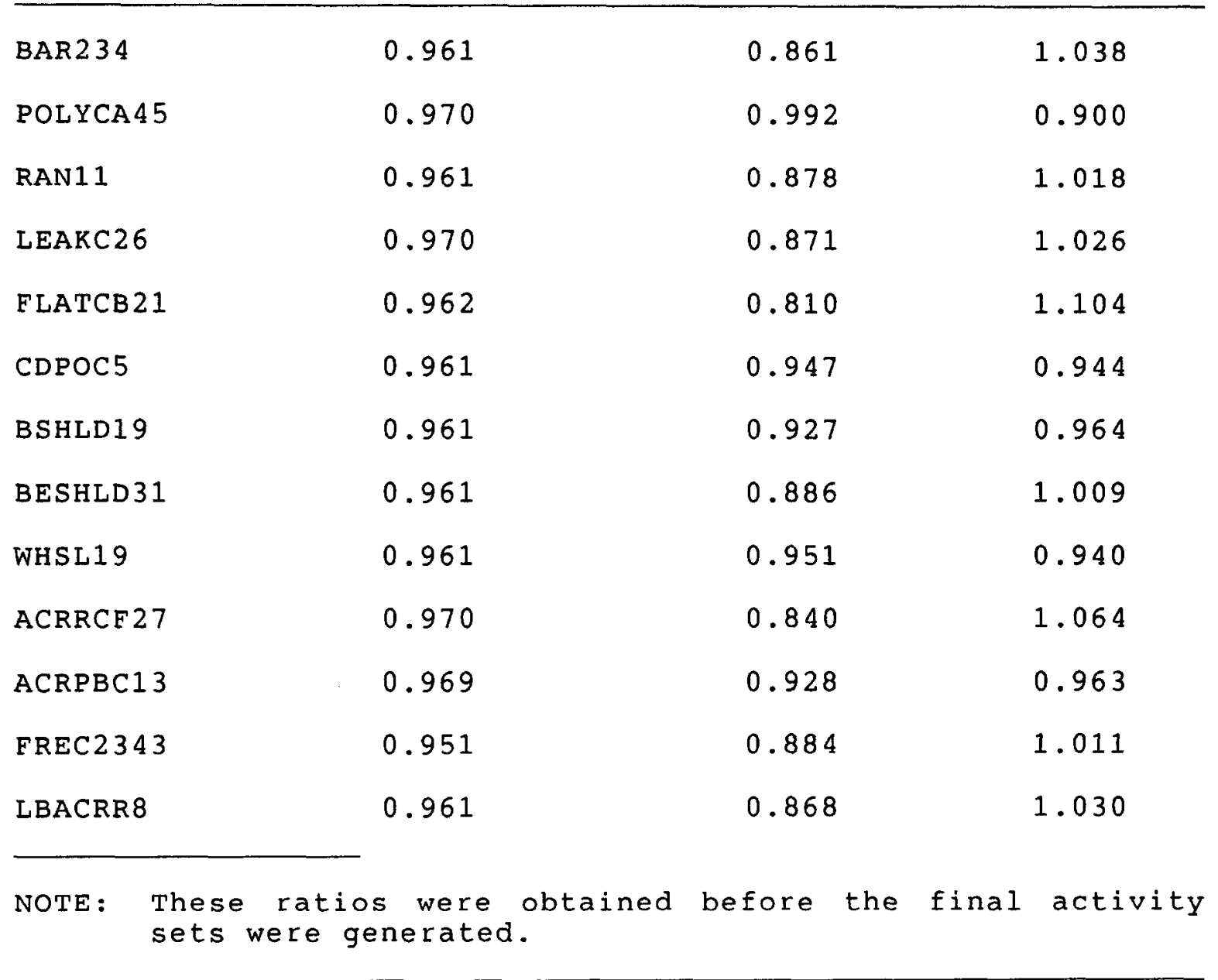




\section{DISTRIBUTION :}

Commander White Sands Missile Range White Sands Missile Range, NM 88002

Attn: J. Meason

STWS-TE-NO

D. W. Weston

Hanford Engineering Development Laboratory

P. O. Box 1970

Richland, WA 99352

J. C. Humphreys

Center for Radiation Research

National Measurement Laboratory

National Bureau of Standards

washington, DC 20234

W. J. Zijp'

Netherlands Energy Research Foundation ECN

P. O. BOX 1

1755 ZG Petten

THE NETHERLANDS

Sam E. Chappel1

Admin., Rm 4625

National Bureau of Standards

Gaithersburg, MD 20899

Stan Wolf

Arcon Corp.

260 Bear Hill Rd.

Waltham, MA 02154

Harry Farrar, IV

Rockwell International

Rocketdyne Division - NA02

6633 Canoga Avenue

Canoga Park, CA 91303

T. Michael Flanders

Nuclear Effects Laboratory

STEWS-TE-NO

White Sands Missile Range

White Sands, NM 88002

Wes Sallee

Nuclear Effects Laboratory

P. O. Box 333

White Sands Missile Range

White sands, NM 88001 


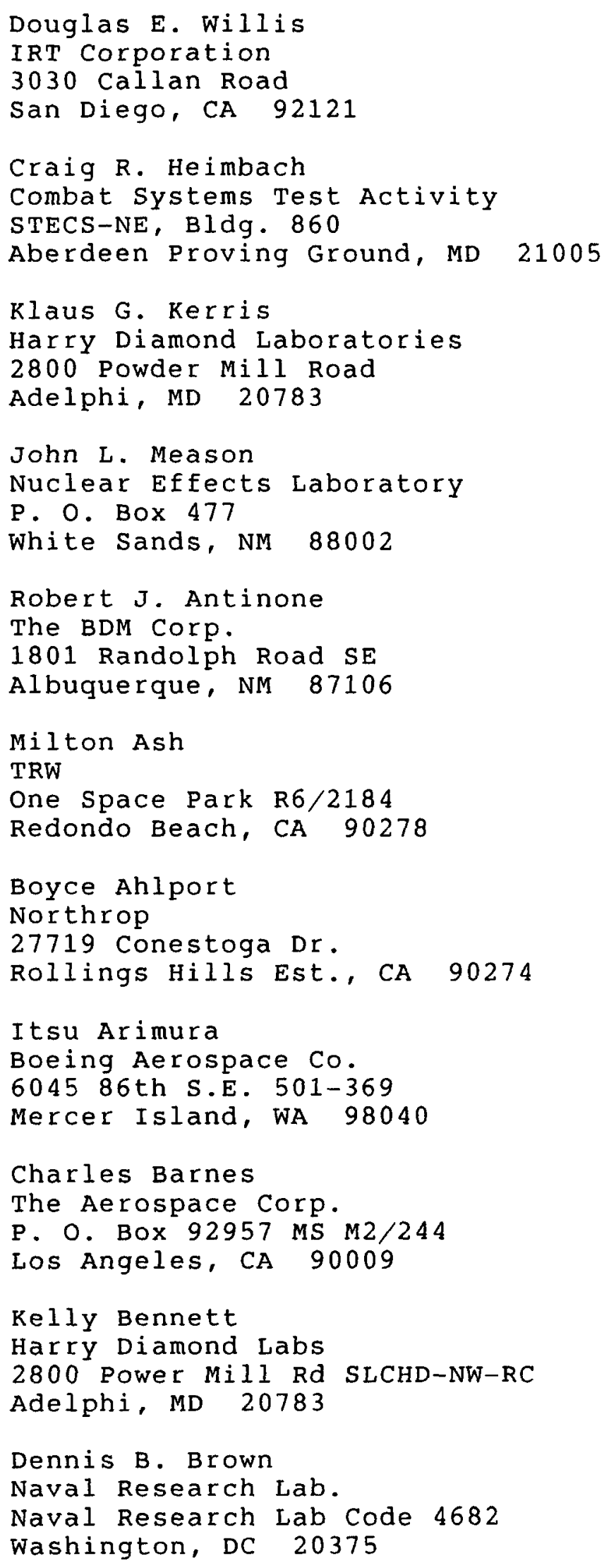


Floyd Coppage

R\&D Associates

P. O. Box 9335

Albuquerque, NM 87119

Charles M. Dozier

Naval Research Lab.

570039 th Ave.

Hyattsville, MD 20781

Thomad D. Ellis

Naval Weapons Support Ctr. - Crane

Rt. \#1 Box 353

Bloomfield, IN 47424

Kenneth F. Galloway

University of Arizona

Bldg. 104

Elec. \& Comp. Eng. DNA Programs

Tucson, Az 85721

John Garth

Rome Air Development Center

23 Morningside Lane Bldg. 725

Lincoln, MA 01773

Mark Haas

TRW

P. O. Box $1310523 / 310$

San Bernardino, CA 92402

John w. Harrity

IRT Corp.

P. O. BOX 85317

San Diego, CA 92138

Chuck Gwyn

United Tech. Microelectronics Ctr.

1575 Garden of the Gods $R$

Colorado Springs, CO 80907

Stephen Judd

C. S. Draper Lab, Inc.

555 Technology Sq. MS51

Cambridge, MA 02139

Klaus Kerris

Harry Diamond Lab

2800 Powder Mill Rd. BR 22900

Adelphi, MD 20783 
Bruce K. Janousek

Aerospace Corp.

P. O. Box 92957 MS $/ 24$

Los Angeles, CA 90009

J. Brian Long

Harris semiconductor

P. O. Box 883 MS53-210

Melbourne, FL 32901

George C. Messenger

Consultant

3111 Bel Air Dr. 7F

Las Vegas, NV 89109

George L. Pease

Mission Research

1720 Randolph Rd. SE

Albuquerque, NM 87106

Randall Pflueger

C. S. Draper Lab, Inc.

555 Technology Sq. MS 51

Cambridge, MA 02139

James P. Raymond

Mission Research Crop.

5434 Ruffin Rd.

San Diego, CA 92123

James L. Ramsey

NWSC Crane

R2 Box 1295152

Odon, IN 47562

Joseph Srour

Northrop Research \& Tech Ctr.

One Research Park 0355/T60

Palos Verdes Peninsula, CA 90274

Virgil H. Strahan

Rockwell International

P. O. BOX 4192 FB12 031-FB12

Anaheim, CA 92803-4192

Eligius Wolicki

Wolicki Associates Inc.

1310 Gatewood Dr. MS12

Alexandria, VA 22307

Edward A. Burke

Mission Research Corp.

11 Indian Hill Rd.

Woburn, MA 01801 


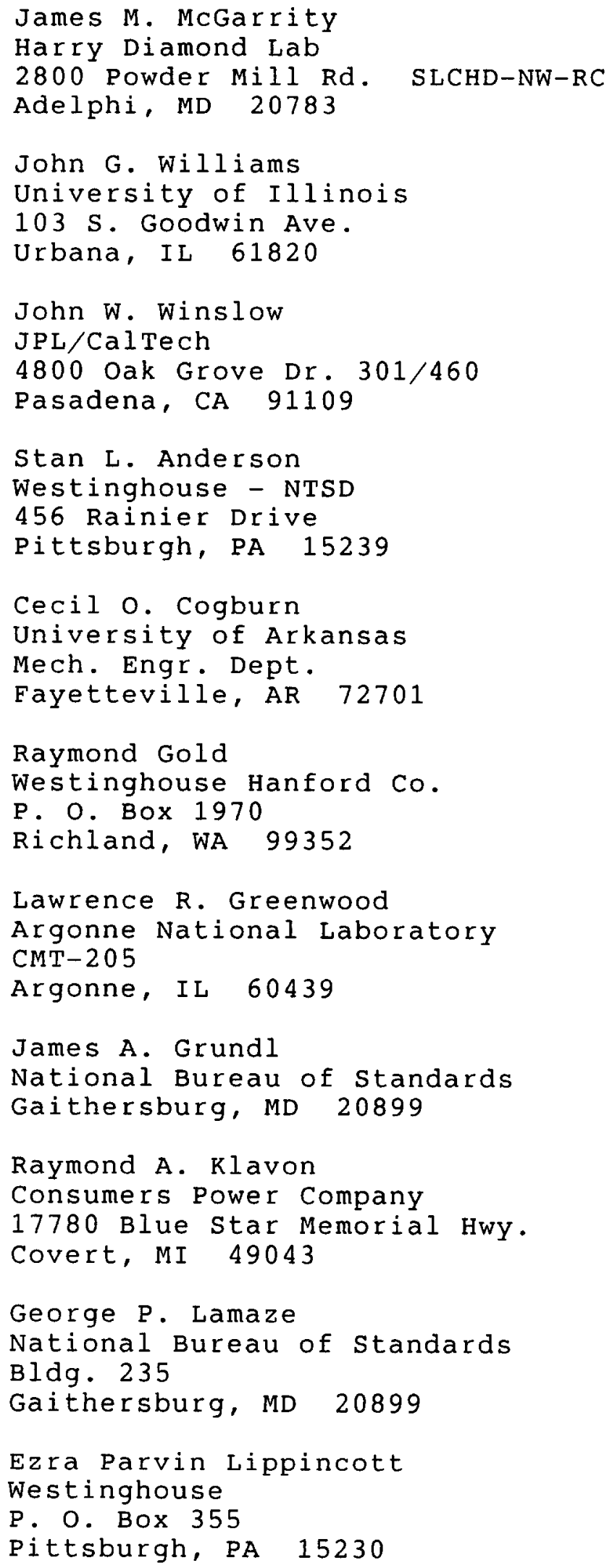


Michael P. Manahan

Battelle

505 King Ave.

Columbus, OH 43201-2693

Wolf Mannhart

PTB Braunschweig

Bundesallee 100

Braunschweig 3300

FEDERAL REPUBLIC OF GERMANY

Gerald C. Martin

General Electric Company

Vallecitos Nuclear Ctr. M/CV04

Pleasanton, CA 94566

William Matsumoto

Westinghouse Hanford Co.

P. O. Box 1970

Bldg. 325, Area $300 \mathrm{~W} / \mathrm{C}-33$

Richland, WA 99352

Manfred Matzke

Physikalisch Tech. Bundesanst.

Bundesallee 100

Braunschweig

FEDERAL REPUBLIC OF GERMANY

William N. MCElroy

Hanford Engr. Develop. Lab

P. O. Box 1970

Richland, WA 99352

E. Dale McGarry

National Bureau of Standards

Gaithersburg, MD 20899

Paul S. Olson

Rockwell International

P. O. Box 1449

Canoga Park, CA 91304

Albert Packwood

UKAEA

AEE Winfrith

Dorchester

Dorset

UNITED KINGDOM DT2 8DH

J. W. Rogers

EG\&G Idaho, Inc.

P. O. Box 1625

Idaho Falls, ID 83415 
Frank H. Ruddy

Westinghouse Research

1310 Beulah Rd.

Pittsburgh, PA 15235

Donald L. Smith

Argonne National Laboratory

Applied Physics, Bldg. 314

Argonne, IL 60439

Friedemann W. Stallmann

Oak Ridge National Laboratory

P. O. Box X, Bldg. 3001 MS-028

Oak Ridge, TN 37831-6028

Jin-Bor Sun

Florida Power \& Light Co.

1801 NW 119 Ave.

Pembroke Pines, FL 33026

Janos Vegh

KFKI, Central Research Inst. for Physics H-1525 Budapest 114

P. O. BOX 49

BUDAPEST

John G. Williams

University of Illinois

219 NEL, $103 \mathrm{~s}$. Goodwin Ave.

Urbana, IL 61801 


\section{SANDIA DISTRIBUTION :}
$1230 \mathrm{~J}$. E. Powell
$1231 \mathrm{~J}$. A. Halbleib
1232 W. Beezhold
1233 L. M. Choate
2126 J. E. Gover
2142 T. A. Fischer
2321 L. D. Posey
2644 H. L. Scott
3423 G. Ramirez
6000 D. L. Hartley
6400 D. J. McCloskey
$6420 \mathrm{~J}$. V. Walker
6421 P. S. Pickard
6421 G. A. Harms
6421 M. S. Lazo
6421 D. A. McArthur
6423 K. O. Reil
6423 R. O. Gauntt
6425 W. J. Camp
6427 M. Berman
6450 T. R. Schmidt
6451 T. F. Luera
6451 D. T. Berry
6451 S. Domingues
6451 C. D. Harmon
6451 D. M. Minnema
6451 F. M. Morris
6452 B. B. Conklin
6452 D. W. Vehar (10)
6454 G. L. Cano
6454 C. V. Holm
6454 J. G. Kelly (25)
6454 A. A. Key
6454 J. B. Wray
7112 F. Biggs
3141 S. A. Landenberger (5)
3151 W. L. Garner (3)
3154 C. H. Dalin (28)
For DOE/OSTI (Unlimited Release)
8524 P. W. Dean 